Supporting Information

\title{
Preparation and Application of Amino Phosphine Ligands Bearing Spiro[indane- 1,2'-pyrrolidine] Backbone
}

Shasha Li, Jinxia Zhang, Hongjie Li, Lifei Feng, Peng Jiao*

Key Laboratory of Radiopharmaceuticals, College of Chemistry, Beijing Normal University, Beijing 100875, P.R. China.

E-mail:pjiao@bnu.edu.cn

\section{Contents}

1. Pd-Catalyzed Enantioselective Allylic Alkylations..................................................................... 1

2. Pd-Catalyzed Enantioselective Allylic Etherification Reactions .................................................S1

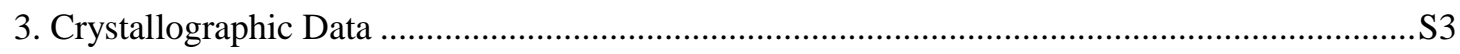

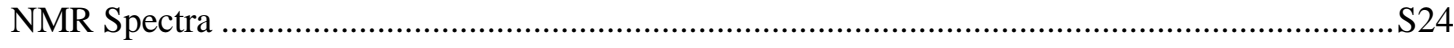

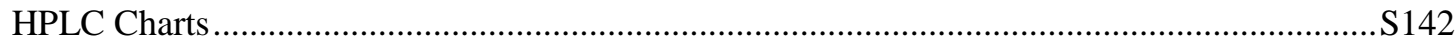




\section{Pd-Catalyzed Enantioselective Allylic Alkylations}

Table S1. Asymmetric allylic alkylations with malonates.

\begin{tabular}{|c|c|c|c|}
\hline & \multicolumn{2}{|c|}{$\begin{array}{l}\mathrm{Pd}_{2}(\mathrm{dba})_{3}(3.0 \text { mol\% }) \\
1 \mathrm{a}(6.0 \mathrm{~mol} \%) \\
\mathrm{CH}_{2}\left(\mathrm{CO}_{2} \mathrm{Me}\right)_{2}(2.0 \text { equiv }) \\
\mathrm{Cs}_{2} \mathrm{CO}_{3}(2.0 \text { equiv })\end{array}$} & \\
\hline \pm$)$ & \multicolumn{2}{|c|}{ solvent, rt, $24 \mathrm{~h}$} & \\
\hline Entry & Solvent & Yield $(\%)^{a}$ & $\operatorname{Ee}(\%)^{a}$ \\
\hline 1 & toluene & 92 & 90 \\
\hline 2 & $\mathrm{Et}_{2} \mathrm{O}$ & 54 & 90 \\
\hline 3 & $\mathrm{THF}$ & $89(83)$ & $91(81)$ \\
\hline 4 & MTBE & $99(80)$ & $92(85)$ \\
\hline 5 & DME & 99 & 87 \\
\hline 6 & 1,4-dioxane & 95 & 91 \\
\hline $7^{b}$ & DCM & $95(85)$ & $70(67)$ \\
\hline 8 & DCE & 95 & 93 \\
\hline $9^{c}$ & DCE & 0 & n.d. \\
\hline
\end{tabular}

${ }^{a}$ Data in parentheses were for products from diethyl malonate. ${ }^{b}$ Diethyl malonate gave $90 \%$ yield and $40 \%$ ee when $\left[\operatorname{PdCl}\left(\eta^{3}-\mathrm{C}_{3} \mathrm{H}_{5}\right)\right]_{2}$ was used. ${ }^{c}$ Ligand $1 e$ was used. The reaction time was $4 \mathrm{~d}$.

\section{Pd-Catalyzed Enantioselective Allylic Etherification Reactions}

Table S2. Asymmetric etherifications with $\mathrm{CH}_{3} \mathrm{OH}$ catalyzed by different ligands.

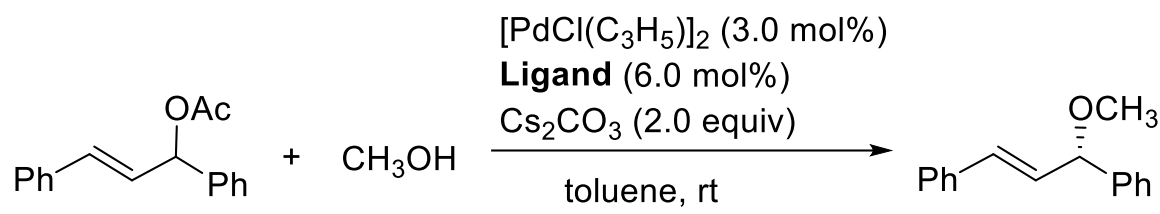

$( \pm)$

\begin{tabular}{cccc}
\hline Entry & Ligand & Yield (\%) & Ee (\%) \\
\hline 1 & 1a & 82 & 94 \\
2 & 1b & 66 & 93 \\
3 & 1c & 81 & 95 \\
4 & 1d & 82 & 97 \\
\hline
\end{tabular}

Table S3. Optimization of the etherification conditions using $\mathrm{CH}_{3} \mathrm{OH}$. 


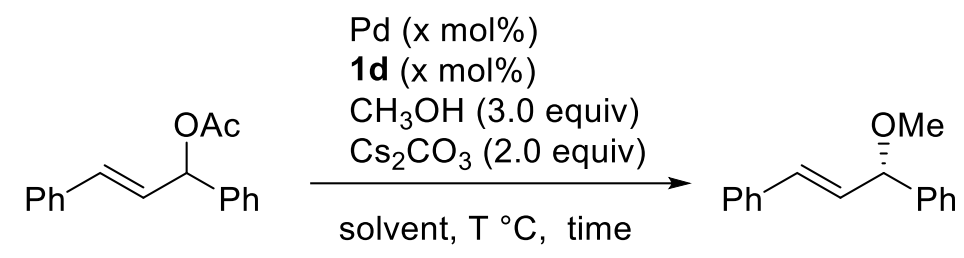

$( \pm)$

yield, ee

\begin{tabular}{lllccccc}
\hline Entry & Solvent & $\mathrm{Pd} \mathrm{source}$ & $\mathrm{x} \mathrm{mol}^{a}{ }^{a}$ & $\mathrm{~T}\left({ }^{\circ} \mathrm{C}\right)$ & Time $(\mathrm{h})$ & Yield $(\%)$ & Ee (\%) \\
\hline 1 & toluene & {$\left[\mathrm{PdCl}\left(\mathrm{C}_{3} \mathrm{H}_{5}\right)\right]_{2}$} & 6 & $\mathrm{rt}$ & 18 & 82 & 97 \\
2 & toluene & $\mathrm{Pd}_{2}(\mathrm{dba})_{3}$ & 6 & $\mathrm{rt}$ & 24 & 0 & n.d. \\
3 & toluene & $\mathrm{Pd}(\mathrm{OAc})_{2}$ & 6 & $\mathrm{rt}$ & 18 & 81 & 87 \\
4 & AcOEt & {$\left[\mathrm{PdCl}\left(\mathrm{C}_{3} \mathrm{H}_{5}\right)\right]_{2}$} & 6 & $\mathrm{rt}$ & 50 & 70 & 97 \\
5 & Et $2 \mathrm{O}$ & {$\left[\mathrm{PdCl}\left(\mathrm{C}_{3} \mathrm{H}_{5}\right)\right]_{2}$} & 6 & $\mathrm{rt}$ & 18 & 80 & 94 \\
6 & $\mathrm{THF}$ & {$\left[\mathrm{PdCl}\left(\mathrm{C}_{3} \mathrm{H}_{5}\right)\right]_{2}$} & 6 & $\mathrm{rt}$ & 18 & 61 & 90 \\
7 & dioxane & {$\left[\mathrm{PdCl}\left(\mathrm{C}_{3} \mathrm{H}_{5}\right)\right]_{2}$} & 6 & $\mathrm{rt}$ & 46 & 65 & 95 \\
8 & $\mathrm{MTBE}$ & {$\left[\mathrm{PdCl}\left(\mathrm{C}_{3} \mathrm{H}_{5}\right)\right]_{2}$} & 6 & $\mathrm{rt}$ & 18 & 72 & 91 \\
9 & DME & {$\left[\mathrm{PdCl}\left(\mathrm{C}_{3} \mathrm{H}_{5}\right)\right]_{2}$} & 6 & $\mathrm{rt}$ & 18 & 70 & 93 \\
10 & DCM & {$\left[\mathrm{PdCl}\left(\mathrm{C}_{3} \mathrm{H}_{5}\right)\right]_{2}$} & 6 & $\mathrm{rt}$ & 18 & 81 & 91 \\
11 & DCE & {$\left[\mathrm{PdCl}\left(\mathrm{C}_{3} \mathrm{H}_{5}\right)\right]_{2}$} & 6 & $\mathrm{rt}$ & 46 & 60 & 38 \\
12 & toluene & {$\left[\mathrm{PdCl}\left(\mathrm{C}_{3} \mathrm{H}_{5}\right)\right]_{2}$} & 10 & $\mathrm{rt}$ & 24 & 92 & 93 \\
13 & toluene & {$\left[\mathrm{PdCl}\left(\mathrm{C}_{3} \mathrm{H}_{5}\right)\right]_{2}$} & 6 & 0 & 18 & 78 & 95 \\
14 & toluene & {$\left[\mathrm{PdCl}\left(\mathrm{C}_{3} \mathrm{H}_{5}\right)\right]_{2}$} & 6 & 40 & 18 & 80 & 90 \\
$15^{b}$ & toluene & {$\left[\mathrm{PdCl}\left(\mathrm{C}_{3} \mathrm{H}_{5}\right)\right]_{2}$} & 6 & $\mathrm{rt}$ & 48 & 0 & n.d. \\
$16^{b}$ & toluene & {$\left[\mathrm{PdCl}\left(\mathrm{C}_{3} \mathrm{H}_{5}\right)\right]_{2}$} & 6 & 40 & 48 & 34 & 31 \\
\hline
\end{tabular}

${ }^{a}$ The mole percent was for monomeric $\mathrm{Pd} .{ }^{b} \mathrm{~K}_{2} \mathrm{CO}_{3}$ was the base. 


\section{Crystallographic Data}

All crystals were grown in the air by slow evaporation of the solvents. Crystals of $\mathbf{1 0}$ and TfNHPh (1:1) were grown in a mixture of hexanes/AcOEt at $4{ }^{\circ} \mathrm{C}$. All the other crystals were grown at rt. Crystals of $\mathbf{4 a}$ were grown in EtOH. Crystals of the debromination product of $\mathbf{4} \mathbf{b}^{\prime}$ were grown in $\mathrm{CH}_{3} \mathrm{OH}$. Crystals of $\mathbf{6 a}, \mathbf{6} \mathbf{b}$ and $\mathbf{1 1 d}$ were grown in AcOEt. Crystals of $\mathbf{2 b}$ were grown in $\mathrm{CH}_{2} \mathrm{Cl}_{2}$. Crystals of $\mathbf{1 a}, \mathbf{8}$ and $\operatorname{HBr}(1: 1), \mathbf{1 1}$ a were grown in $\mathrm{CDCl}_{3}$. Crystals of the enantiomer of $\mathbf{5}$, racemic $\mathbf{6 b}, \mathbf{8}$ and $N$-[(S,E)-1,3-diphenylallyl]-( $R$ )-phenylglycinol were grown in a mixture of hexanes/AcOEt. Crystals of 9 were grown in a mixture of THF/AcOEt. Crystals of [Pd(II) $\left(\eta^{3}-\right.$ $\mathrm{PhCHCHCHPh})(\mathbf{1 d})] \mathrm{PF}_{6}$ were grown in a mixture of $n$-hexane $/ \mathrm{CH}_{2} \mathrm{Cl}_{2}$.

Compound 1a (The ellipsoid contour percent probability level is 50\%.)

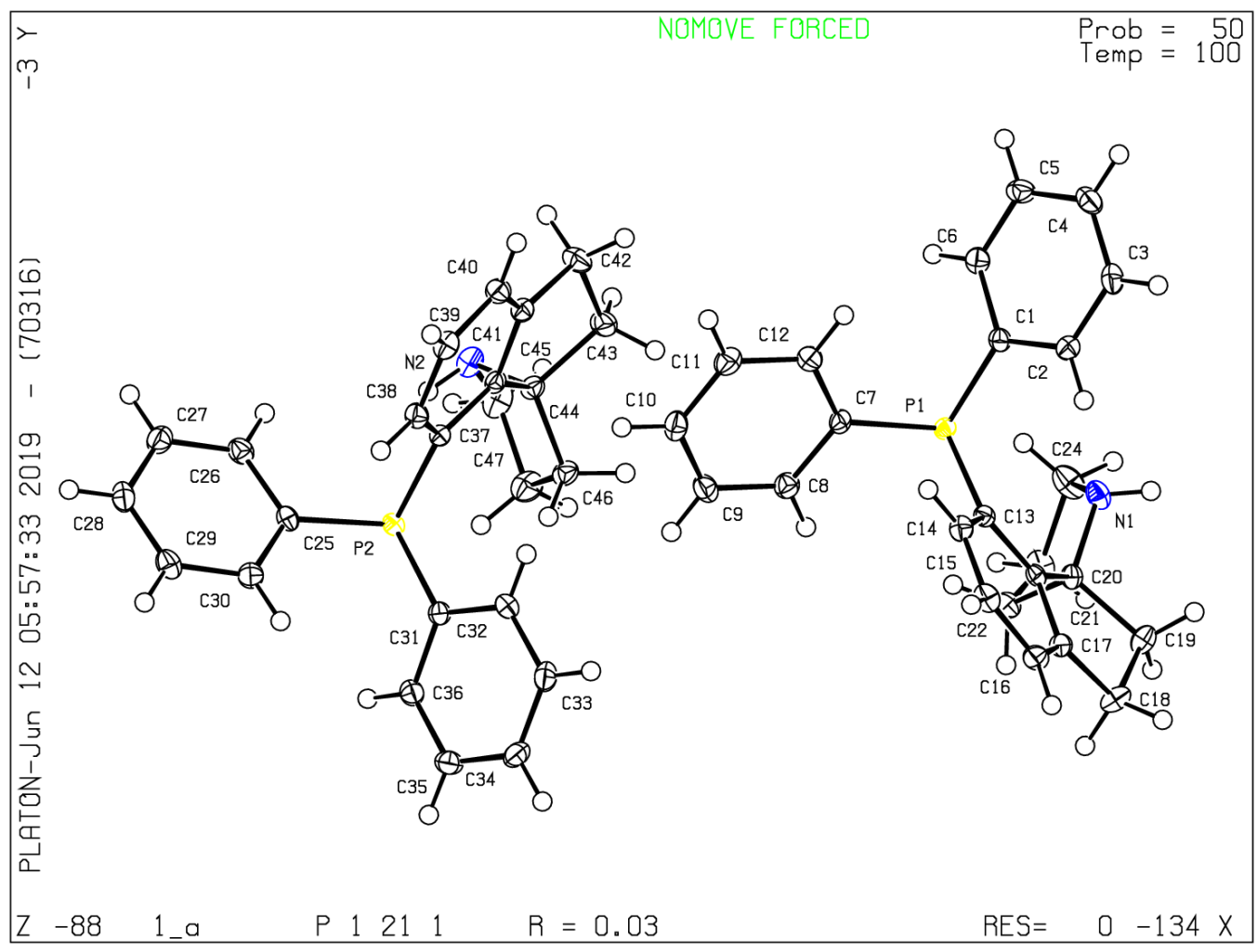

Bond precision:

Cell:

$$
\begin{aligned}
& a=11.6263(1) \\
& \text { alpha }=90
\end{aligned}
$$

Temperature: $100 \mathrm{~K}$

Calculated

1843.05(3)

Volume

P 21

P 2yb

C24 H24 N P

Space group

Hall group

Moiety formula
Wavelength $=1.54178$

$c=11.7477(1)$

gamma $=90$ $b=13.4946(1)$

beta $=90.512(1)$
Reported

1843.05(3)

P 1211

P 2yb

C24 H24 N P 


\begin{tabular}{|c|c|c|}
\hline Sum formula & C24 H24 N P & $\mathrm{C} 24 \mathrm{H} 24 \mathrm{~N} \mathrm{P}$ \\
\hline $\mathrm{Mr}$ & 357.41 & 357.41 \\
\hline Dx,g cm-3 & 1.288 & 1.288 \\
\hline $\mathrm{Z}$ & 4 & 4 \\
\hline $\mathrm{Mu}(\mathrm{mm}-1)$ & 1.351 & 1.351 \\
\hline F000 & 760.0 & 760.0 \\
\hline F000' & 762.94 & \\
\hline $\mathrm{h}, \mathrm{k}, \operatorname{lmax}$ & $14,16,14$ & $14,16,14$ \\
\hline Nref & $7423[3878]$ & 7182 \\
\hline Tmin,Tmax & $0.850,0.874$ & \\
\hline Tmin' & 0.763 & \\
\hline \multicolumn{3}{|l|}{ Correction method $=$ Not given } \\
\hline Data completeness $=1.85 / 0.97$ & \multirow{2}{*}{\multicolumn{2}{|c|}{$\begin{array}{l}\operatorname{Theta}(\max )=73.214 \\
\quad \text { wR2(reflections })=0.0669(7182)\end{array}$}} \\
\hline $\mathrm{R}($ reflections $)=0.0253(7031)$ & & \\
\hline $\mathrm{S}=1.037$ & Npar $=469$ & \\
\hline
\end{tabular}

Compound 2b (The ellipsoid contour percent probability level is 50\%.)

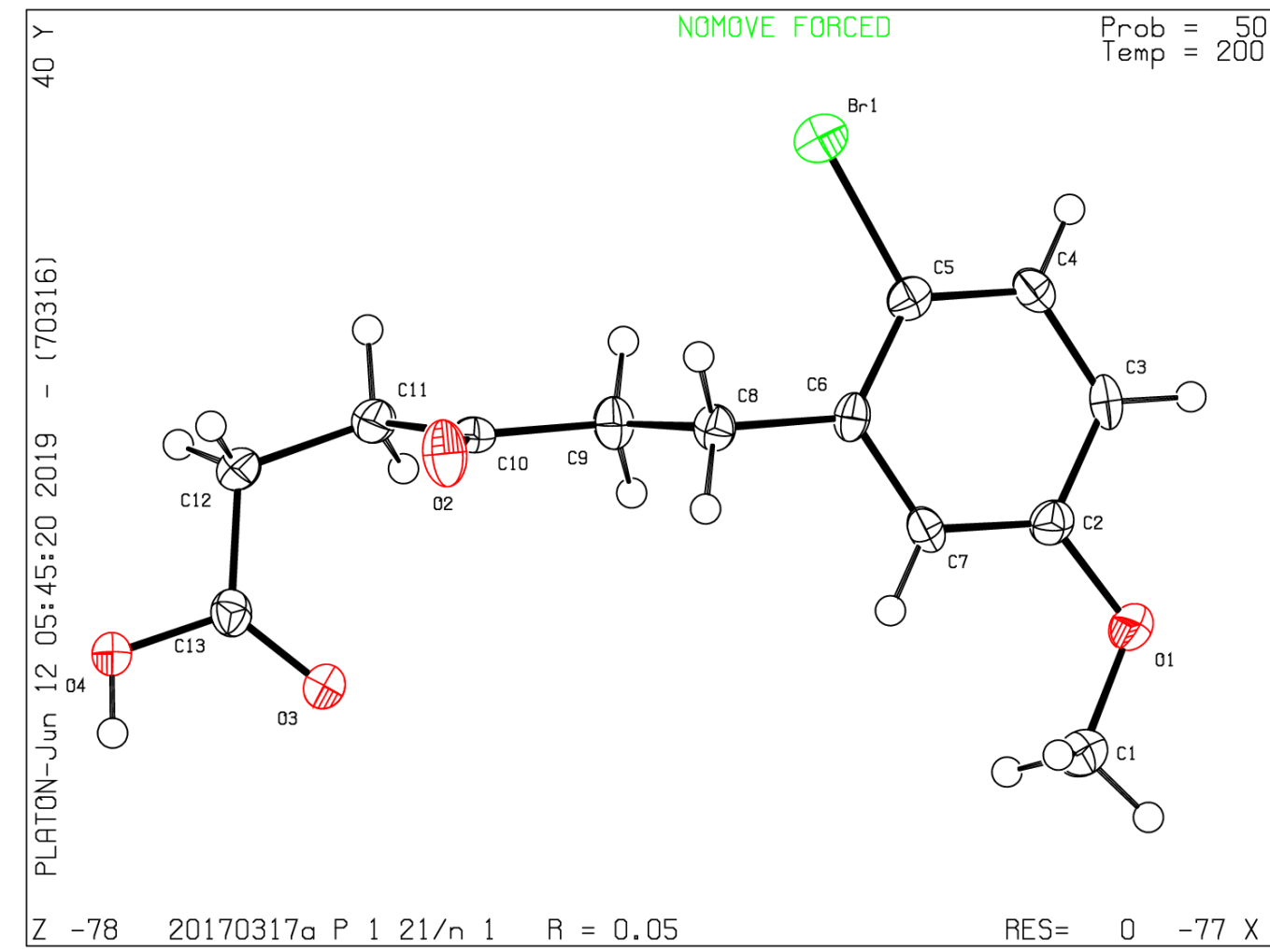

Bond precision:

$\mathrm{C}-\mathrm{C}=0.0050 \mathrm{~A}$

Wavelength $=0.71073$

Cell:

$\mathrm{a}=9.7187(9)$

$\mathrm{b}=5.5986(5)$

$\mathrm{c}=23.953(3)$ 
alpha $=90 \quad$ beta $=99.621(11) \quad$ gamma $=90$

Temperature: $200 \mathrm{~K}$

\begin{tabular}{|c|c|c|}
\hline & Calculated & Reported \\
\hline Volume & $1285.0(2)$ & $1285.0(2)$ \\
\hline Space group & P 21/n & P $121 / n 1$ \\
\hline Hall group & $-\mathrm{P} 2 \mathrm{yn}$ & $-\mathrm{P} 2 \mathrm{yn}$ \\
\hline Moiety formula & $\mathrm{C} 13 \mathrm{H} 15 \mathrm{Br} \mathrm{O} 4$ & $\mathrm{C} 13 \mathrm{H} 15 \mathrm{Br} \mathrm{O} 4$ \\
\hline Sum formula & C13 H15 Br O4 & C13 H15 Br O4 \\
\hline $\mathrm{Mr}$ & 315.15 & 315.16 \\
\hline Dx,g cm-3 & 1.629 & 1.629 \\
\hline Z & 4 & 4 \\
\hline $\mathrm{Mu}(\mathrm{mm}-1)$ & 3.203 & 3.203 \\
\hline F000 & 640.0 & 640.0 \\
\hline F000' & 639.18 & \\
\hline $\mathrm{h}, \mathrm{k}, \operatorname{lmax}$ & $13,7,33$ & $13,7,32$ \\
\hline Nref & 3656 & 3087 \\
\hline Tmin,Tmax & $0.398,0.726$ & $0.668,1.000$ \\
\hline Tmin' & 0.368 & \\
\hline \multicolumn{3}{|c|}{ Correction method= \# Reported T Limits: Tmin=0.668 Tmax=1.000 AbsCorr $=$ MULTI-SCAN } \\
\hline Data completene & \multicolumn{2}{|c|}{$\operatorname{Theta}(\max )=29.732$} \\
\hline $\mathrm{R}($ reflections $)=($ & \multicolumn{2}{|c|}{$\mathrm{wR} 2($ reflections $)=0.1019(3087)$} \\
\hline$S=1.067$ & \multicolumn{2}{|l|}{ Npar $=165$} \\
\hline
\end{tabular}

Compound 4a (The ellipsoid contour percent probability level is 50\%.) 


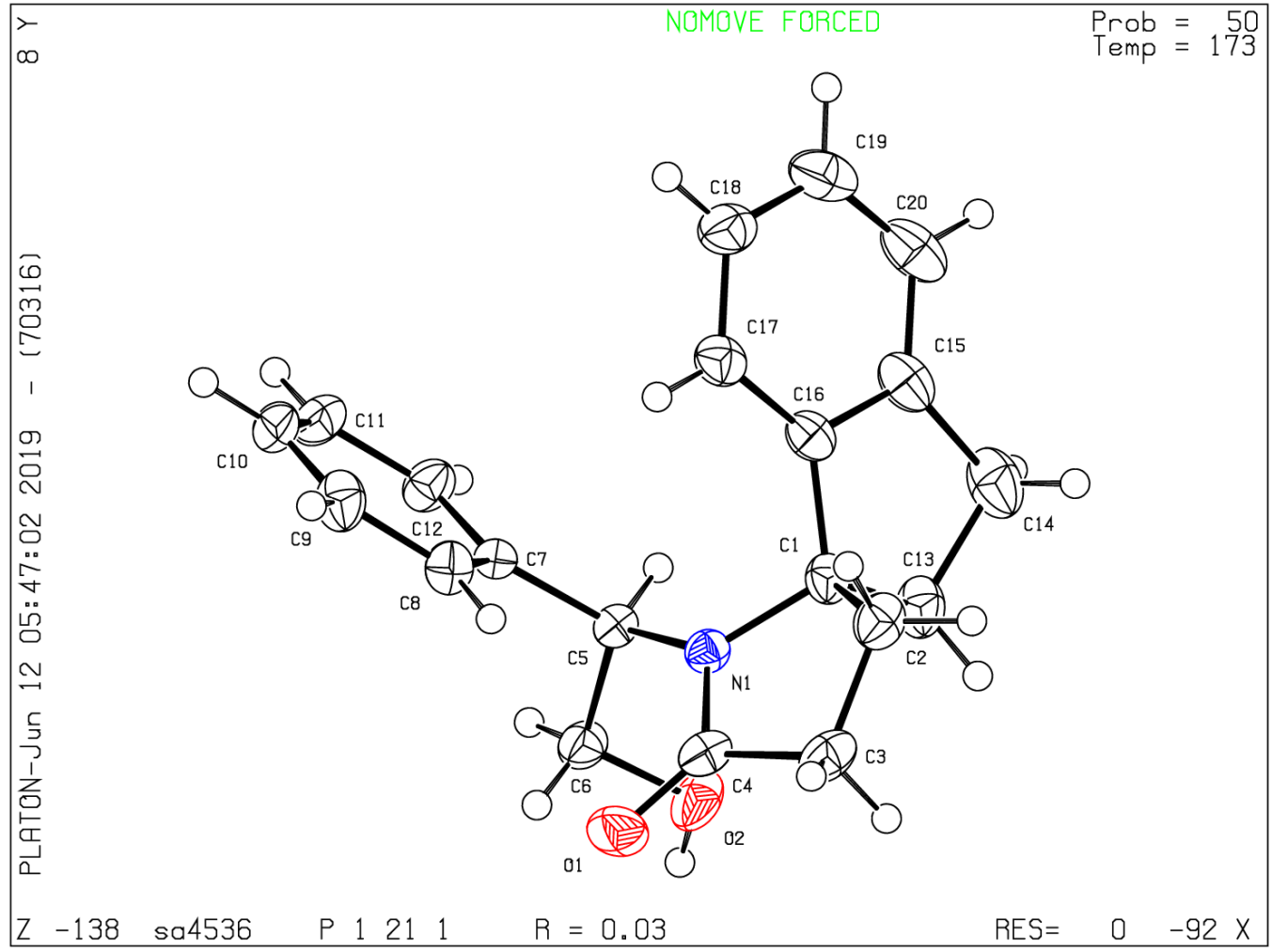

Bond precision:

$\mathrm{C}-\mathrm{C}=0.0031 \mathrm{~A}$

Wavelength $=0.71073$

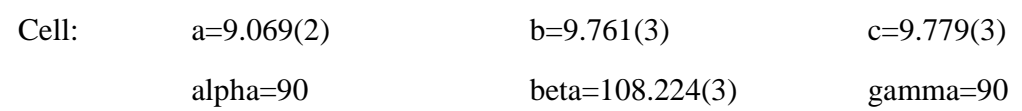

Temperature: $173 \mathrm{~K}$

Calculated

Volume

Space group

Hall group

Moiety formula

Sum formula

$\mathrm{Mr}$

Dx,g cm-3

$\mathrm{Z}$

$\mathrm{Mu}(\mathrm{mm}-1)$

F000

F000'

h,k,lmax

Nref

Tmin,Tmax

Tmin'
822.2(4)

P 21

$\mathrm{P} 2 \mathrm{yb}$

C20 H21 N O2

$\mathrm{C} 20 \mathrm{H} 21 \mathrm{~N} \mathrm{O} 2$

307.38

1.242

2

0.080

328.0

328.14

$11,12,12$

3779[ 2001]

$0.972,0.986$

0.972

\section{Reported}

822.2(4)

P 1211

P 2yb

C20 H21 N O2

C20 H21 N O2

307.38

1.242

2

0.080

328.0

$11,12,12$

3716

$0.893,1.000$ 
Correction method $=$ \# Reported T Limits: Tmin=0.893 Tmax=1.000 AbsCorr $=$ MULTI-SCAN

Data completeness $=1.86 / 0.98$

$\mathrm{R}($ reflections $)=0.0340(3623)$

$\mathrm{S}=1.052$
Theta $(\max )=27.484$

$\mathrm{wR} 2($ reflections $)=0.0803(3716)$

Npar $=212$

Debromination product of compound 4b' (The ellipsoid contour percent probability level is $50 \%$.)

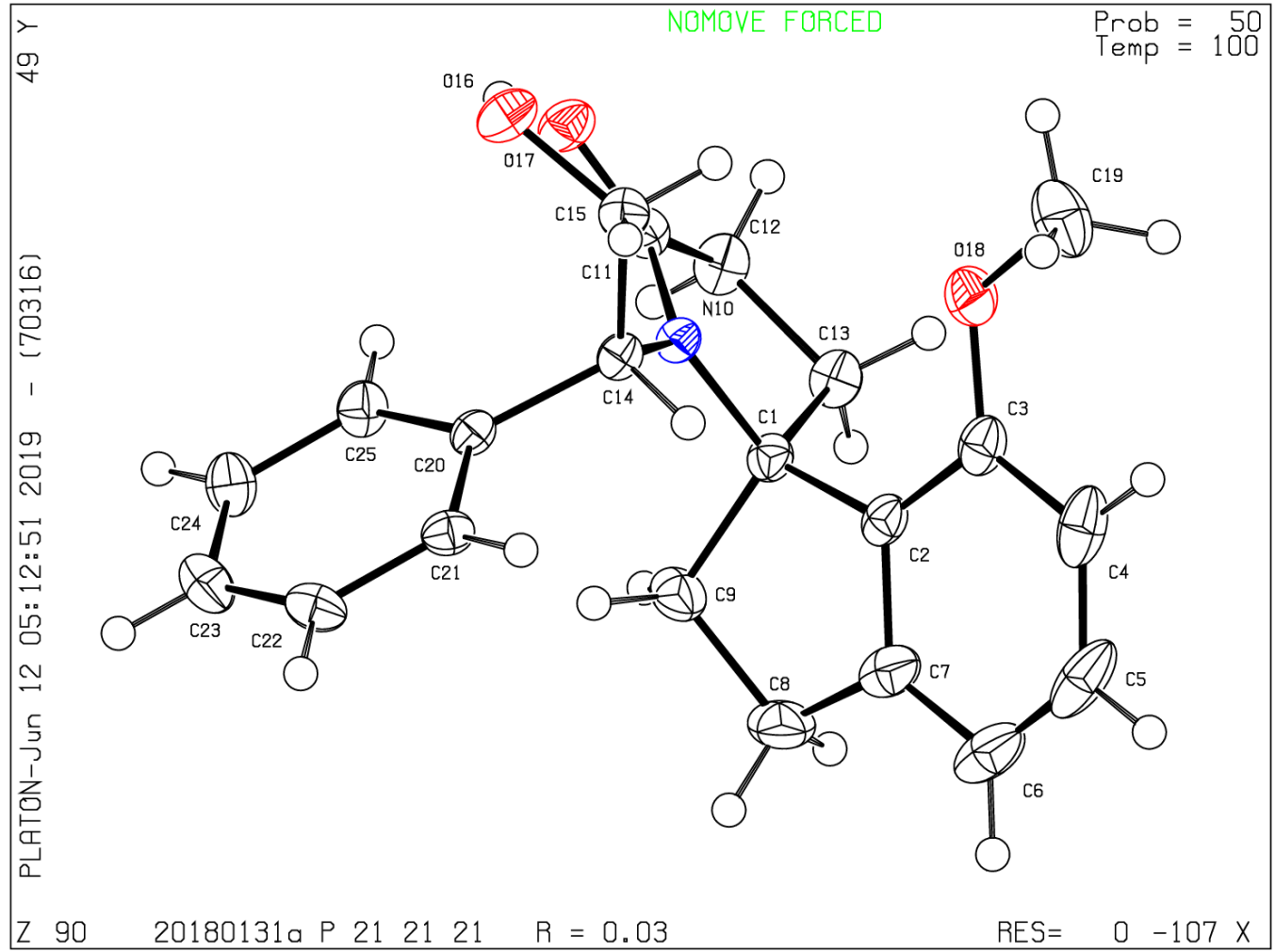

Bond precision:

Cell:

$$
\begin{aligned}
& a=7.6720(1) \\
& \text { alpha }=90
\end{aligned}
$$$$
\mathrm{C}-\mathrm{C}=0.0024 \mathrm{~A}
$$$$
\mathrm{b}=15.0220(2)
$$

beta $=90$

Calculated

1797.97(4)

P 212121

P 2ac 2ab

C21 H23 N O3

$\mathrm{C} 21 \mathrm{H} 23 \mathrm{~N} \mathrm{O} 3$

337.40

1.247

4

0.665

\section{Dx,g cm-3}

$\mathrm{Z}$

$\mathrm{Mu}(\mathrm{mm}-1)$
Wavelength $=1.54184$

$c=15.6008(2)$

gamma $=90$
Reported

1797.97(4)

P 212121

P 2ac 2ab

C21 H23 N O3

C21 H23 N O3

337.40

1.246

4

0.665 
F000'

h,k,lmax

Nref

Tmin,Tmax

Tmin'
722.14

$9,18,19$

$9,18,19$

3607[ 2075]

3507

$0.805,0.819$

$0.894,1.000$

0.730

Correction method $=$ \# Reported T Limits: Tmin $=0.894$ Tmax $=1.000$ AbsCorr $=$ MULTI-SCAN

Data completeness $=1.69 / 0.97$

$\mathrm{R}($ reflections $)=0.0302(3425)$

$\mathrm{S}=1.074$
$\operatorname{Theta}(\max )=73.185$

$w R 2($ reflections $)=0.0759(3507)$

Npar $=228$

Enantiomer of compound 5a (The ellipsoid contour percent probability level is 50\%.)

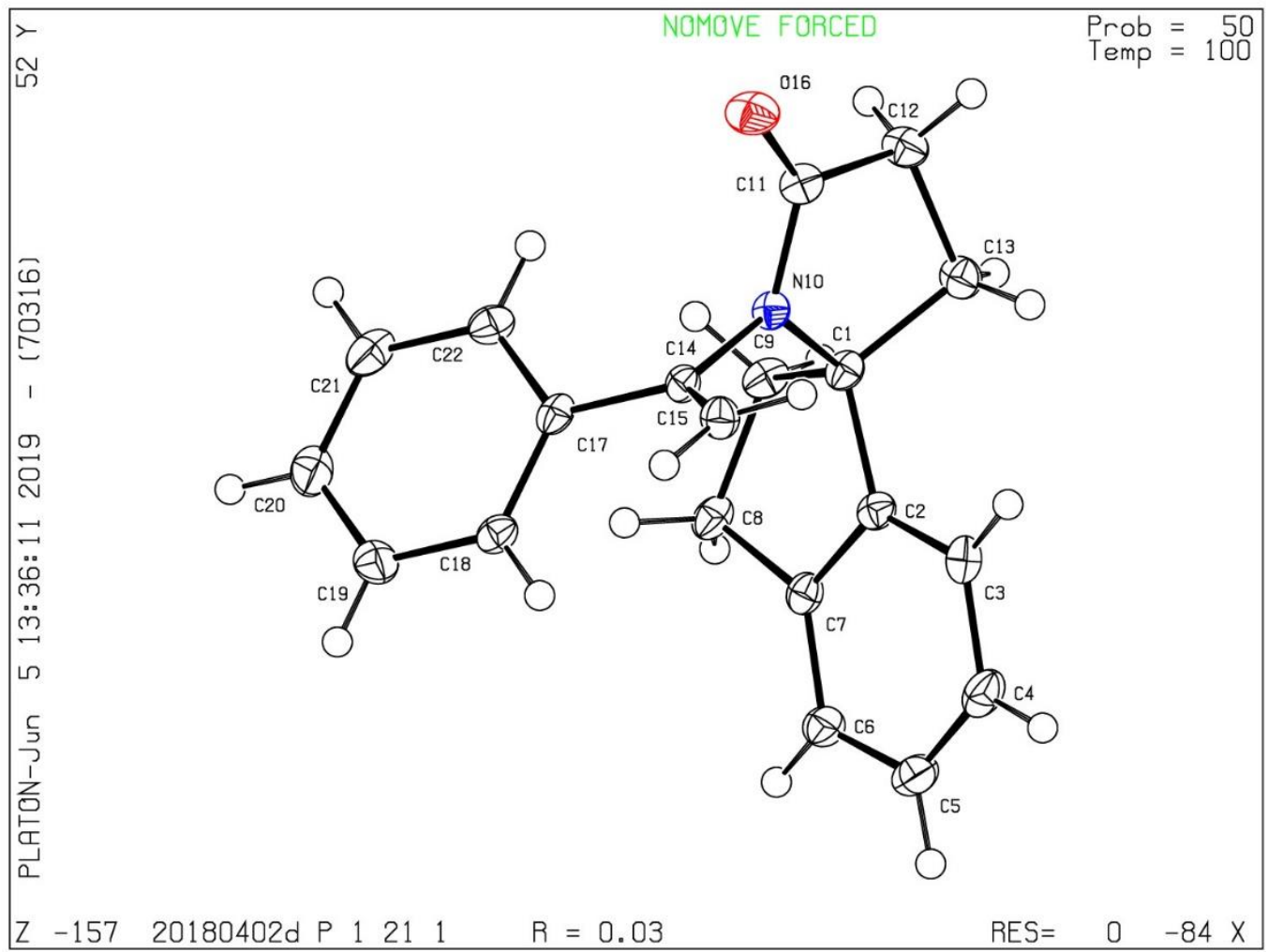

Bond precision:

$\mathrm{C}-\mathrm{C}=0.0045 \mathrm{~A}$

Wavelength $=1.54184$

Cell: $\quad \begin{aligned} & \mathrm{a}=7.0120(3) \\ & \mathrm{alpha}=90\end{aligned}$

$\mathrm{b}=8.2202(3)$

$c=13.1458(6)$

Temperature: $100 \mathrm{~K}$

beta $=90.185(4)$

gamma $=90$

Calculated

Volume

Space group

Hall group
757.72(5)

P 21

P 2yb
Reported

757.72(5)

P 1211

$\mathrm{P} 2 \mathrm{yb}$ 


\begin{tabular}{|c|c|c|}
\hline Moiety formula & C20 H19 N O & C20 H19 N O \\
\hline Sum formula & C20 H19 N O & C20 H19 N O \\
\hline $\mathrm{Mr}$ & 289.36 & 289.36 \\
\hline Dx,g cm-3 & 1.268 & 1.268 \\
\hline $\mathrm{Z}$ & 2 & 2 \\
\hline $\mathrm{Mu}(\mathrm{mm}-1)$ & 0.604 & 0.604 \\
\hline F000 & 308.0 & 308.0 \\
\hline F000' & 308.84 & \\
\hline $\mathrm{h}, \mathrm{k}, \operatorname{lmax}$ & $8,9,15$ & $8,9,15$ \\
\hline Nref & $2818[1515]$ & 2643 \\
\hline Tmin,Tmax & $0.865,0.886$ & $0.886,1.000$ \\
\hline Tmin' & 0.834 & \\
\hline \multicolumn{3}{|c|}{ Correction method $=$ \# Reported T Limits: Tmin $=0.886$ Tmax $=1.000$ AbsCorr $=$ MULTI-SCAN } \\
\hline \multicolumn{2}{|c|}{ Data completeness $=1.74 / 0.94$} & $\operatorname{Theta}(\max )=68.850$ \\
\hline \multicolumn{2}{|c|}{$\mathrm{R}($ reflections $)=0.0349(2629)$} & wR2(reflections) $=0.0943(2643)$ \\
\hline $\mathrm{S}=1.085$ & \multicolumn{2}{|l|}{ Npar $=200$} \\
\hline
\end{tabular}

Compound 6a (The ellipsoid contour percent probability level is 50\%.)

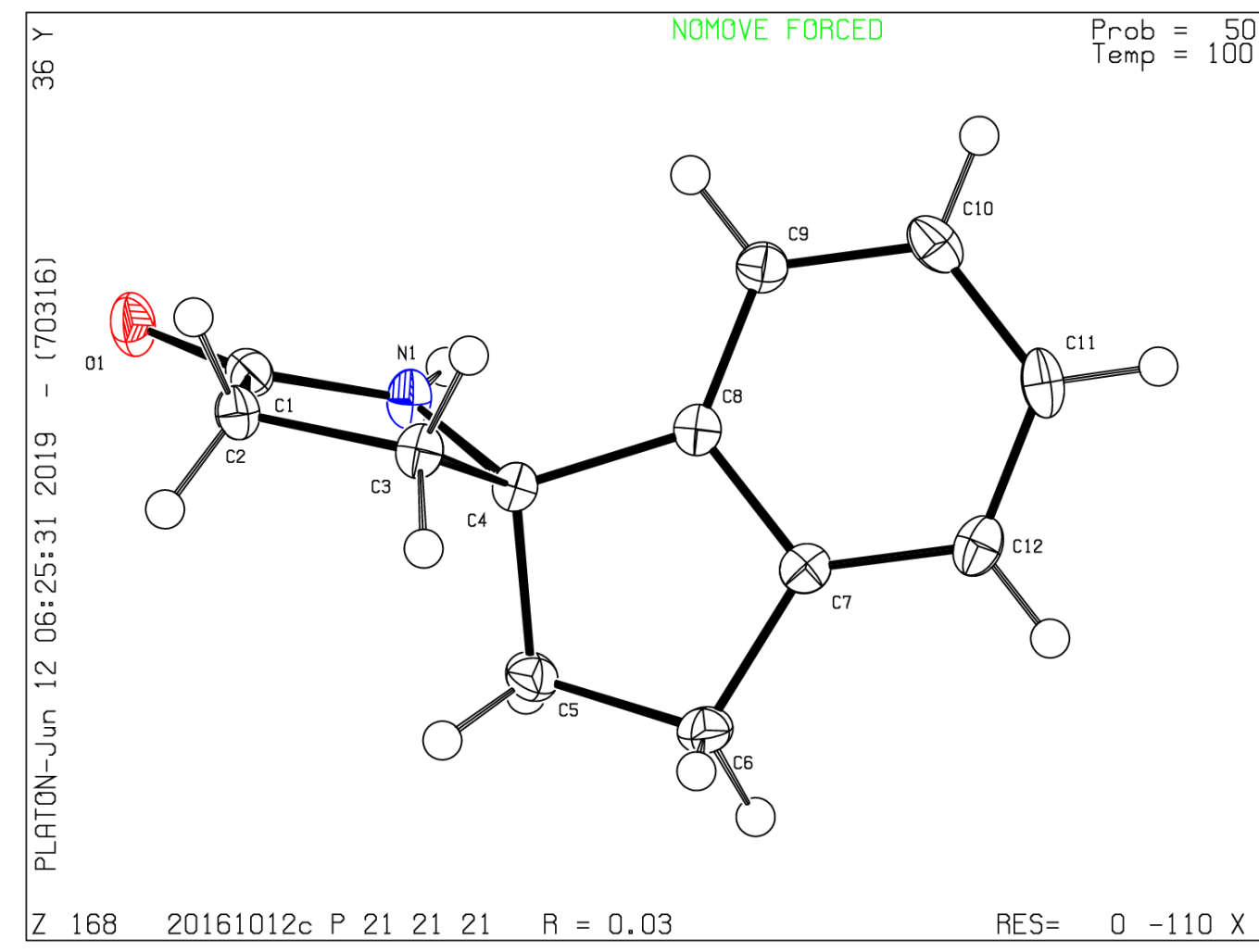

Bond precision:

$\mathrm{C}-\mathrm{C}=0.0021 \mathrm{~A}$

Wavelength $=1.54184$

Cell: $\quad \mathrm{a}=7.39581(12) \quad \mathrm{b}=9.46599(17) \quad \mathrm{c}=13.8475(2)$ 

alpha $=90$
beta $=90$
gamma $=90$

Temperature: $100 \mathrm{~K}$

\begin{tabular}{|c|c|c|}
\hline & Calculated & Reported \\
\hline Volume & $969.45(3)$ & $969.44(3)$ \\
\hline Space group & P 212121 & P 212121 \\
\hline Hall group & P $2 a c 2 a b$ & P $2 a c 2 a b$ \\
\hline Moiety formula & C12 H13 N O & C12 H13 N O \\
\hline Sum formula & C12 H13 N O & C12 H13 N O \\
\hline $\mathrm{Mr}$ & 187.23 & 187.23 \\
\hline Dx,g cm-3 & 1.283 & 1.283 \\
\hline $\mathrm{Z}$ & 4 & 4 \\
\hline $\mathrm{Mu}(\mathrm{mm}-1)$ & 0.645 & 0.645 \\
\hline F000 & 400.0 & 400.0 \\
\hline F000' & 401.13 & \\
\hline h,k, lmax & $9,11,17$ & $9,11,16$ \\
\hline Nref & 1946[ 1147] & 1884 \\
\hline Tmin,Tmax & $0.824,0.908$ & $0.894,1.000$ \\
\hline Tmin' & 0.824 & \\
\hline \multicolumn{3}{|c|}{ Correction method $=\#$ Reported T Limits: Tmin $=0.894$ Tmax=1.000 AbsCorr $=$ MULTI-SCAN } \\
\hline \multicolumn{2}{|c|}{ Data completeness $=1.64 / 0.97$} & $\operatorname{Theta}(\max )=73.011$ \\
\hline \multicolumn{2}{|c|}{$\mathrm{R}($ reflections $)=0.0312(1872)$} & wR2(reflections) $=0.0811(1884)$ \\
\hline$S=1.132$ & \multicolumn{2}{|l|}{ Npar $=127$} \\
\hline
\end{tabular}

Compound $\mathbf{6 b}$ (The ellipsoid contour percent probability level is $50 \%$.) 


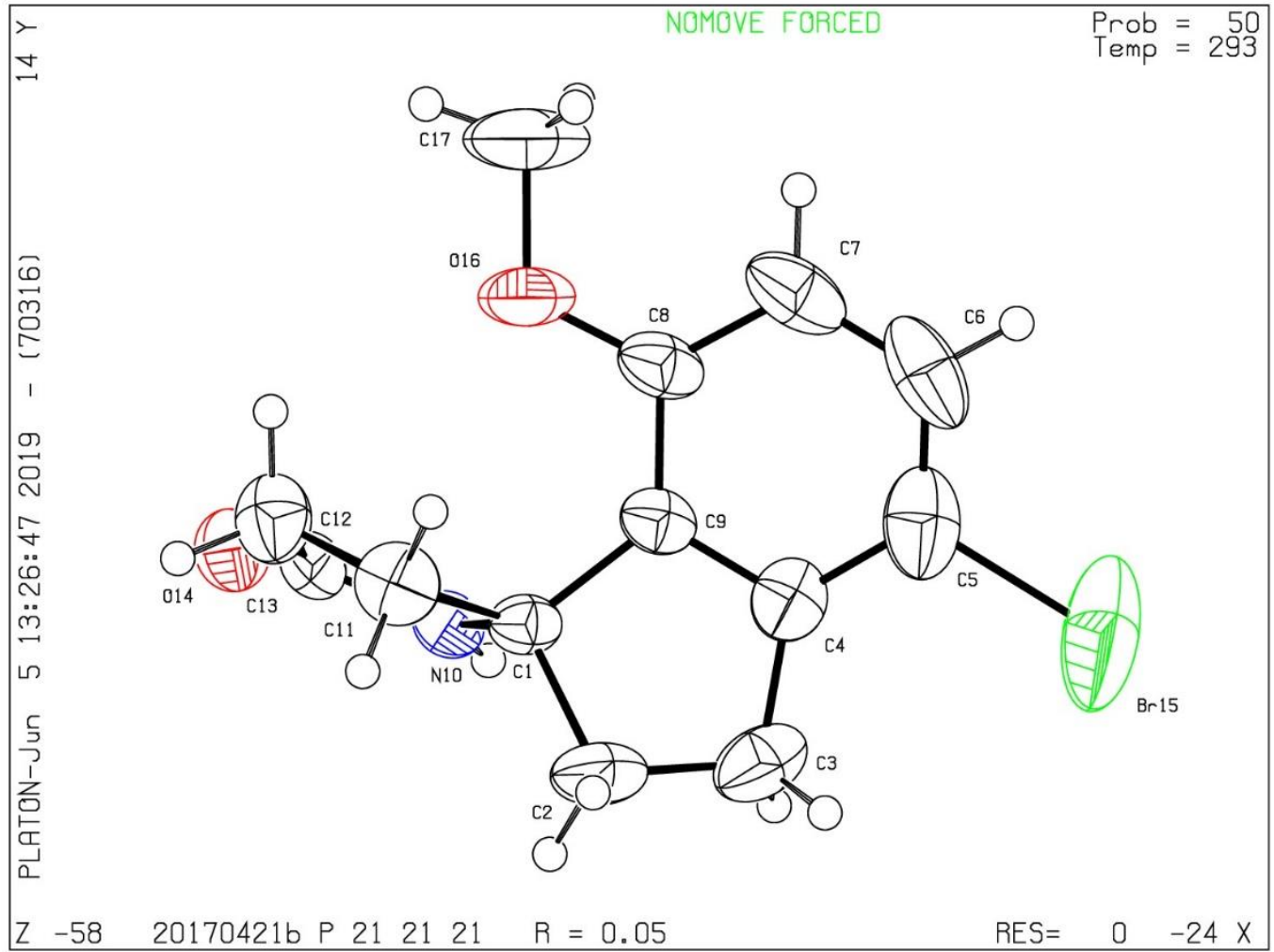

Bond precision:

$\mathrm{C}-\mathrm{C}=0.0086 \mathrm{~A}$

Wavelength $=1.54184$

Cell: $\quad a=8.0131(2)$

$b=8.5786(2)$

$c=18.3195(5)$

alpha $=90$

beta $=90$

gamma $=90$

Temperature: $293 \mathrm{~K}$

Calculated

Volume

Space group

Hall group

Moiety formula

Sum formula

$\mathrm{Mr}$

Dx, $\mathrm{g} \mathrm{cm}-3$

$\mathrm{Z}$

$\mathrm{Mu}(\mathrm{mm}-1)$

F000

F000'

h,k,lmax

Nref

Tmin,Tmax

Tmin'
1259.30(6)

P 212121

P 2ac 2ab

C13 H14 Br N O2

C13 H14 Br N O2

296.15

1.562

4

4.370

600.0

598.69

$9,10,22$

2520[ 1474]

$0.337,0.270$

0.216

\section{Reported}

1259.30(6)

P 212121

P 2ac 2ab

C13 H14 Br N O2

C13 H14 Br N O2

296.16

1.562

4

4.370

600.0

$9,10,22$

2176

$0.505,1.000$ 
Correction method $=$ \# Reported T Limits: Tmin=0.505 Tmax=1.000 AbsCorr $=$ MULTI-SCAN

Data completeness $=1.48 / 0.86$

$\mathrm{R}($ reflections $)=0.0535(2022)$

$\mathrm{S}=1.065$
$\operatorname{Theta}(\max )=73.214$

$w R 2($ reflections $)=0.1569(2176)$

Npar $=155$

Racemic 6b (The ellipsoid contour percent probability level is 50\%.)

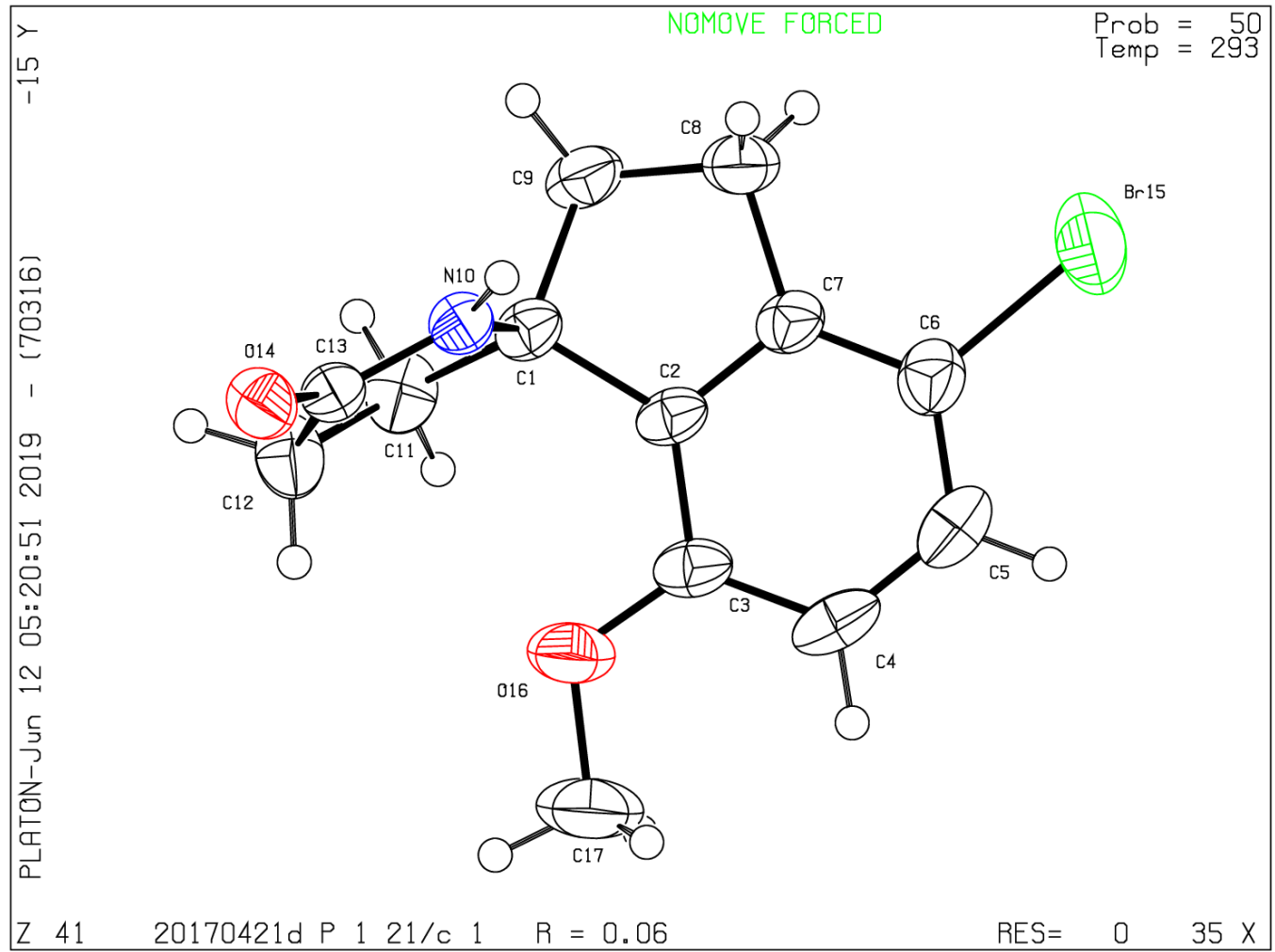

Bond precision:

Cell: $a=8.4333(2)$
alpha $=90$

alpha=90

Temperature: $293 \mathrm{~K}$

\begin{tabular}{lll} 
& Calculated & \multicolumn{1}{c}{ Reported } \\
Volume & $1259.67(4)$ & $1259.67(4)$ \\
Space group & $\mathrm{P} 21 / \mathrm{c}$ & $\mathrm{P} 121 / \mathrm{c} 1$ \\
Hall group & $-\mathrm{P} 2 \mathrm{ybc}$ & $-\mathrm{P} 2 \mathrm{ybc}$ \\
Moiety formula & $\mathrm{C} 13 \mathrm{H} 14 \mathrm{Br} \mathrm{N} \mathrm{O} 2$ & $\mathrm{C} 13 \mathrm{H} 14 \mathrm{Br} \mathrm{N} \mathrm{O2}$ \\
Sum formula & $\mathrm{C} 13 \mathrm{H} 14 \mathrm{Br} \mathrm{N} \mathrm{O} 2$ & $\mathrm{C} 13 \mathrm{H} 14 \mathrm{Br} \mathrm{N} \mathrm{O2}$ \\
$\mathrm{Mr}$ & 296.15 & 296.16 \\
$\mathrm{Dx}, \mathrm{g} \mathrm{cm}-3$ & 1.562 & 1.562 \\
$\mathrm{Z}$ & 4 & 4 \\
$\mathrm{Mu}(\mathrm{mm}-1)$ & 4.369 & 4.369
\end{tabular}


F000

600.0

600.0

F000'

598.69

$\mathrm{h}, \mathrm{k}, \mathrm{lmax}$

$10,18,13$

$10,17,13$

Nref

2539

2458

Tmin,Tmax

$0.403,0.646$

$0.537,1.000$

Tmin'

0.234

Correction method $=$ \# Reported T Limits: Tmin $=0.537$ Tmax $=1.000$ AbsCorr $=$ MULTI-SCAN

Data completeness $=0.968$

$\operatorname{Theta}(\max )=73.349$

$\mathrm{R}($ reflections $)=0.0570(2234)$

$w R 2$ (reflections) $=0.1566(2458)$

$\mathrm{S}=1.039$

Npar $=155$

Compound 8 (The ellipsoid contour percent probability level is 50\%.)

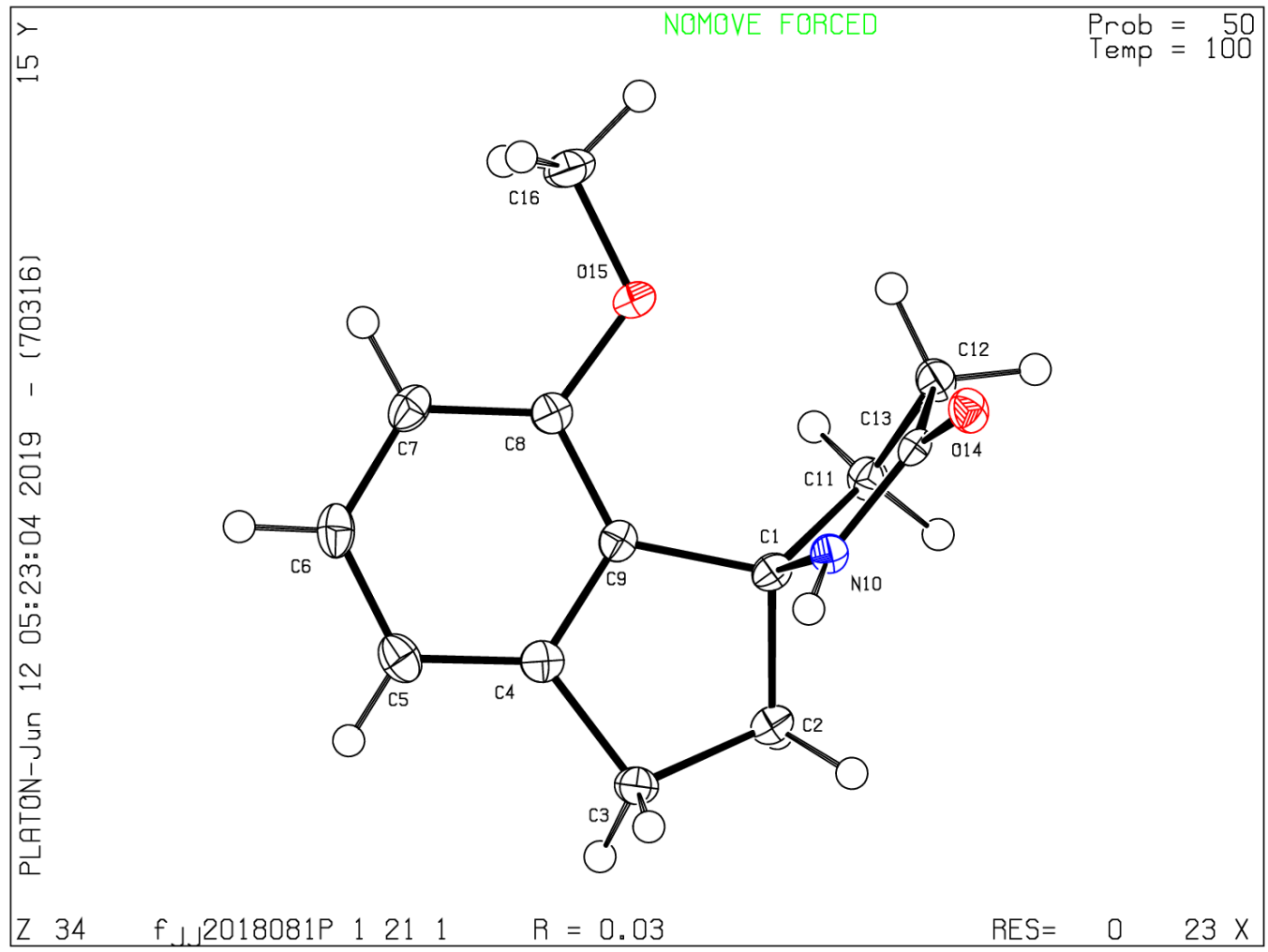

Bond precision:

$\mathrm{C}-\mathrm{C}=0.0021 \mathrm{~A}$

Wavelength $=1.54184$
Cell: $\quad \mathrm{a}=8.0054(1)$
alpha $=90$
$b=7.7314(1)$
beta $=95.249(1)$

$\mathrm{c}=8.6884(1)$

gamma $=90$

Temperature: $100 \mathrm{~K}$

$$
\text { Calculated }
$$

$535.496(11)$

P 21

$\mathrm{P} 2 \mathrm{yb}$
Reported

535.496(11)

P 1211

P 2yb 


$\begin{array}{lll}\text { Moiety formula } & \mathrm{C} 13 \mathrm{H} 15 \mathrm{~N} \mathrm{O} 2 & \mathrm{C} 13 \mathrm{H} 15 \mathrm{~N} \mathrm{O} 2 \\ \text { Sum formula } & \mathrm{C} 13 \mathrm{H} 15 \mathrm{~N} \mathrm{O} 2 & \mathrm{C} 13 \mathrm{H} 15 \mathrm{~N} \mathrm{O} 2 \\ \mathrm{Mr} & 217.26 & 217.26 \\ \mathrm{Dx}, \mathrm{g} \mathrm{cm}-3 & 1.347 & 1.347 \\ \mathrm{Z} & 2 & 2 \\ \text { Mu (mm-1) } & 0.732 & 0.732 \\ \text { F000 } & 232.0 & 232.0 \\ \text { F000' } & 232.69 & \\ \text { h,k,lmax } & 9,9,10 & 9,9,10 \\ \text { Nref } & 2039[1100] & 1979 \\ \text { Tmin,Tmax } & 0.803,0.864 & 0.624,1.000 \\ \text { Tmin' } & 0.746 & \\ \text { Correction method=\# Reported T Limits: Tmin=0.624 Tmax=1.000 AbsCorr = MULTI-SCAN } \\ \text { Data completeness=1.80/0.97 } \\ \text { R(reflections)=0.0269( 1961) } \\ \text { S = 1.103 }\end{array}$

Compound 8 and $\operatorname{HBr}$ (1:1) (The ellipsoid contour percent probability level is 50\%.)

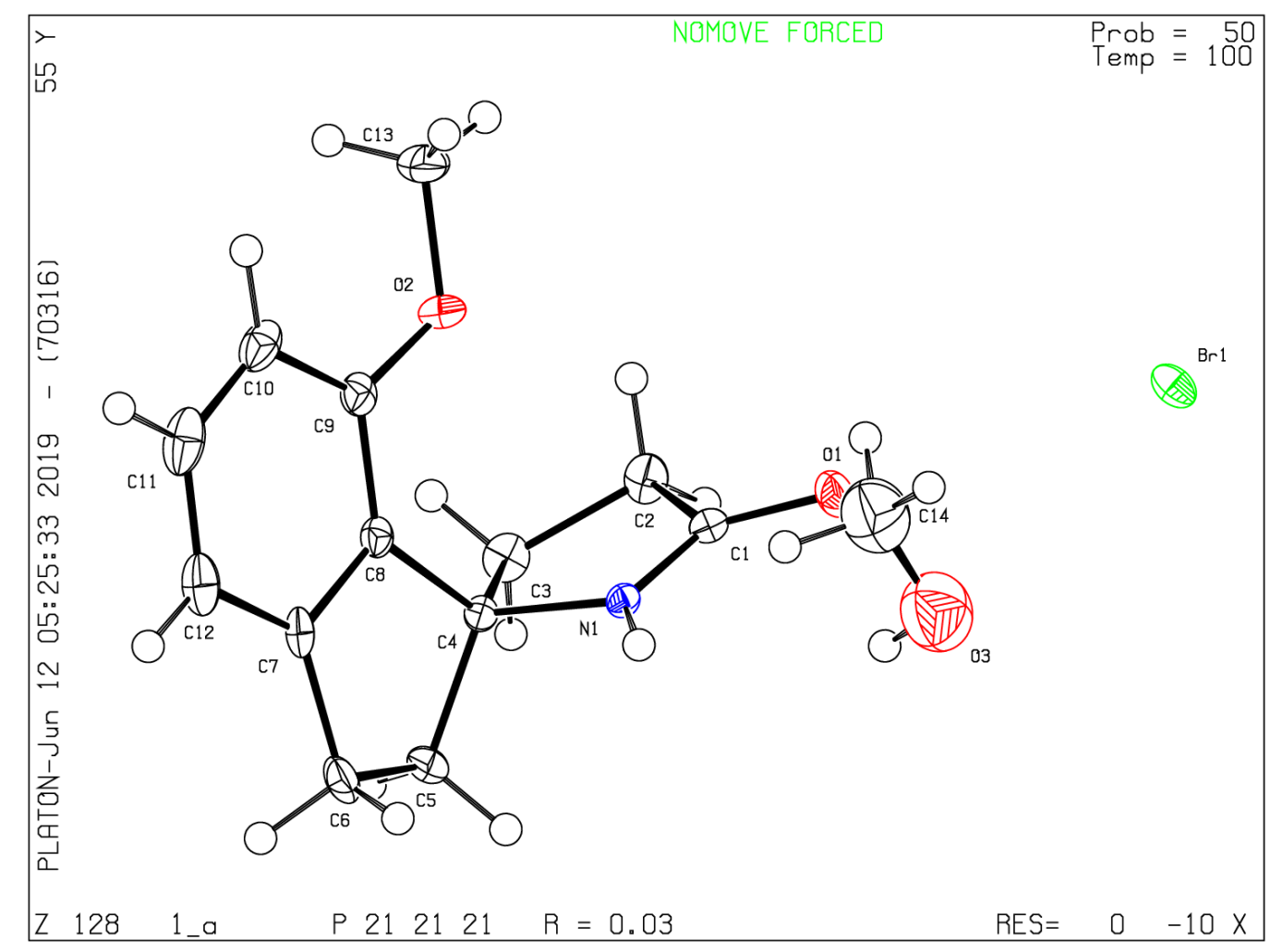

Bond precision:

$\mathrm{C}-\mathrm{C}=0.0069 \mathrm{~A}$

Wavelength $=1.54178$

Cell: $\quad \mathrm{a}=8.0696(1) \quad \mathrm{b}=10.1608(1) \quad \mathrm{c}=16.8229(2)$ 

alpha $=90$
beta $=90$
gamma $=90$

Temperature: $100 \mathrm{~K}$

\begin{tabular}{|c|c|c|}
\hline & Calculated & Reported \\
\hline Volume & $1379.37(3)$ & $1379.37(3)$ \\
\hline Space group & P 212121 & P 212121 \\
\hline Hall group & P $2 a c 2 a b$ & P $2 \mathrm{ac} 2 \mathrm{ab}$ \\
\hline Moiety formula & C13 H15 N O2, C H4 O, Br & $\mathrm{Br}, \mathrm{C} 13 \mathrm{H} 15 \mathrm{~N} \mathrm{O} 2, \mathrm{C} \mathrm{H} 4 \mathrm{O}$ \\
\hline Sum formula & C14 H19 Br N O3 & C14 H19 Br N O3 \\
\hline $\mathrm{Mr}$ & 329.20 & 329.21 \\
\hline Dx,g cm-3 & 1.585 & 1.585 \\
\hline $\mathrm{Z}$ & 4 & 4 \\
\hline $\mathrm{Mu}(\mathrm{mm}-1)$ & 4.105 & 4.105 \\
\hline F000 & 676.0 & 676.0 \\
\hline F000' & 674.95 & \\
\hline $\mathrm{h}, \mathrm{k}, \operatorname{lmax}$ & $9,12,20$ & $9,12,20$ \\
\hline Nref & $2706[1573]$ & 2656 \\
\hline Tmin,Tmax & $0.262,0.292$ & $0.410,1.000$ \\
\hline Tmin' & 0.168 & \\
\hline \multicolumn{3}{|c|}{ Correction method $=\#$ Reported T Limits: Tmin=0.410 Tmax=1.000 AbsCorr $=$ MULTI-SCAN } \\
\hline \multicolumn{2}{|l|}{ Data completeness $=1.69 / 0.98$} & \\
\hline \multicolumn{2}{|l|}{$\mathrm{R}($ reflections $)=0.0316(2647)$} & $2(2656)$ \\
\hline$S=1.114$ & Npar $=174$ & \\
\hline
\end{tabular}

Compound 9 (The ellipsoid contour percent probability level is $50 \%$.) 


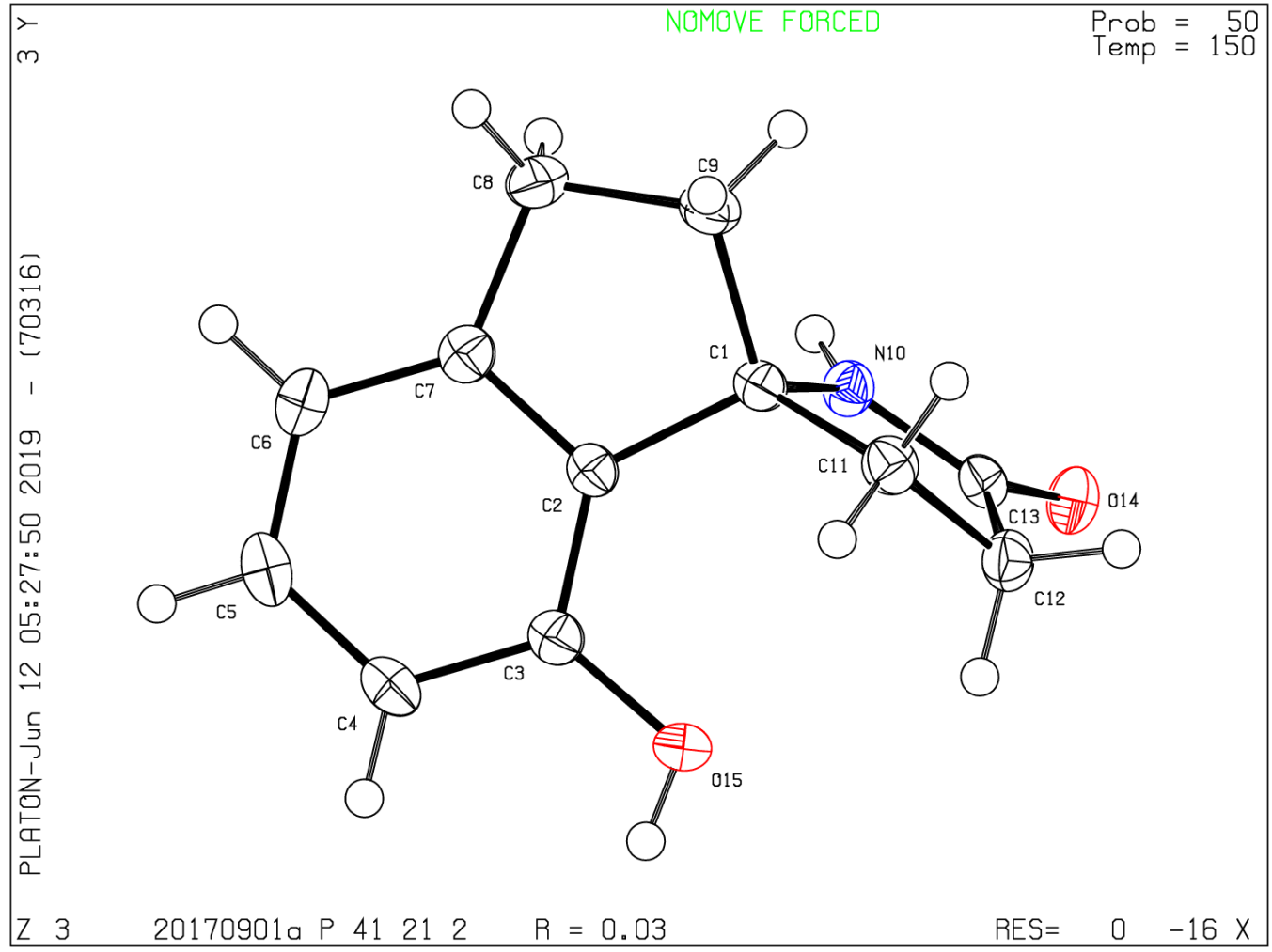

Bond precision:

$\mathrm{C}-\mathrm{C}=0.0021 \mathrm{~A}$

Wavelength $=1.54184$

Cell: $a=12.2380(1)$
alpha=90

$\mathrm{b}=12.2380(1)$

$c=13.7036(2)$

$150 \mathrm{~K}$

Temperature: $150 \mathrm{~K}$

\section{Calculated}

Volume

Space group

Hall group

Moiety formula

Sum formula

$\mathrm{Mr}$

Dx, $\mathrm{g} \mathrm{cm}-3$

$\mathrm{Z}$

$\mathrm{Mu}(\mathrm{mm}-1)$

F000

F000'

h,k,lmax

Nref

Tmin,Tmax

Tmin'
2052.37(4)

P 41212

P 4abw 2nw

C12 H13 N O2

C12 H13 N O2

203.23

1.316

8

0.728

864.0

866.64

$15,15,17$

2057[ 1236]

$0.769,0.865$

0.747
Reported

2052.37(4)

P 41212

P 4abw 2nw

C12 H13 N O2

C12 H13 N O2

203.23

1.315

8

0.728

864.0

$15,12,16$

2035

$0.659,1.000$ 
Correction method $=$ \# Reported T Limits: Tmin=0.659 Tmax=1.000 AbsCorr $=$ MULTI-SCAN

Data completeness $=1.65 / 0.99$

$\mathrm{R}($ reflections $)=0.0284(2002)$

$\mathrm{S}=1.078$
$\operatorname{Theta}(\max )=73.238$

$w R 2($ reflections $)=0.0708(2035)$

Npar $=137$

Compound 10 and PhNHTf (1:1) (The ellipsoid contour percent probability level is 50\%.)

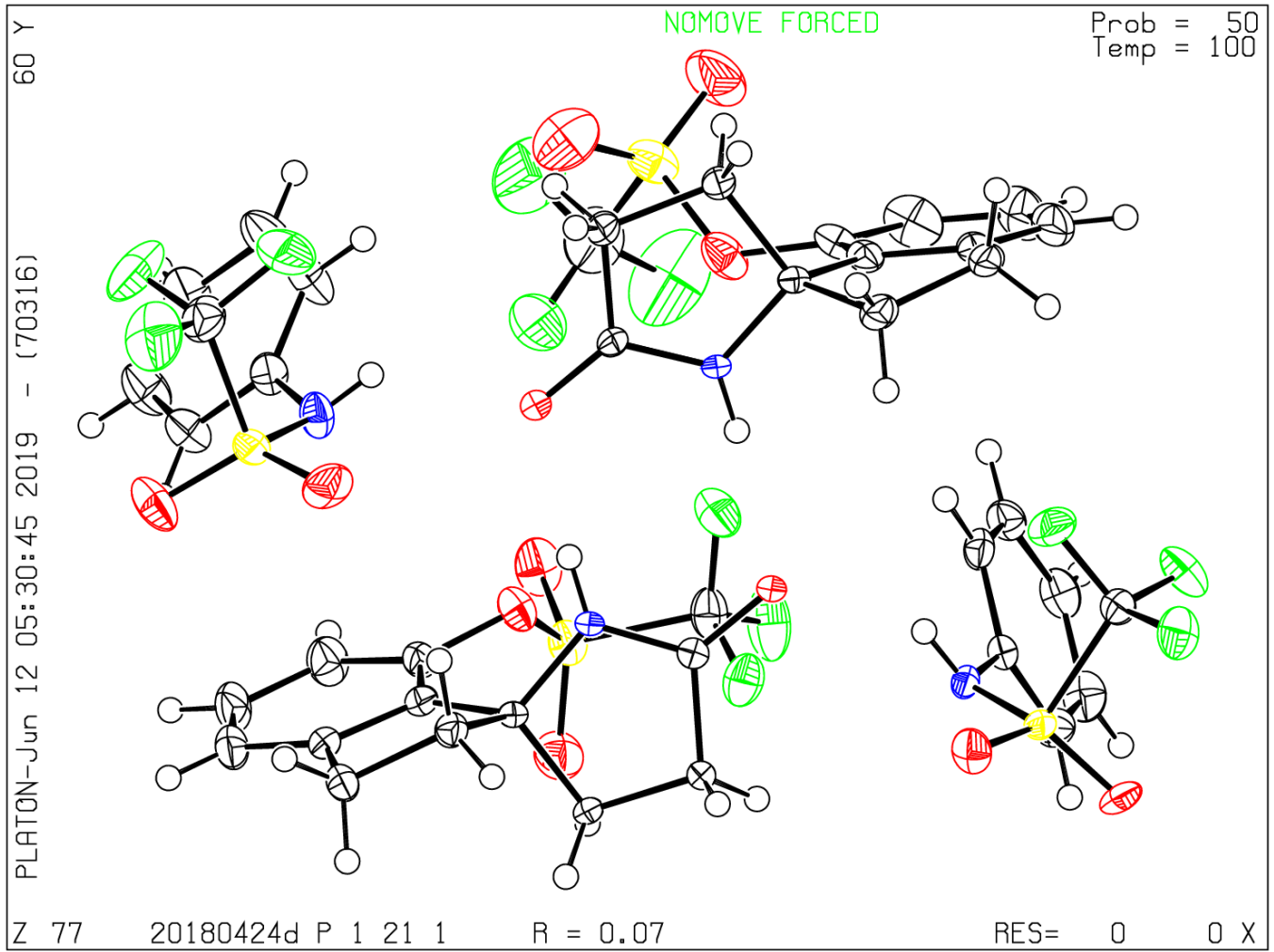

Bond precision:

Cell: $\mathrm{a}=9.2626(1)$
alpha $=90$

Temperature: $100 \mathrm{~K}$

$\begin{array}{ll}\text { Volume } & \begin{array}{l}\text { Calculated } \\ 2363.69(4)\end{array} \\ \text { Space group } & \text { P 21 } \\ \text { Hall group } & \text { P 2yb } \\ \text { Moiety formula } & \text { C13 H12 F3 N O4 S, C7 H6 F3 N O2 S } \\ \text { Sum formula } & \text { C20 H18 F6 N2 O6 S2 } \\ \text { Mr } & 560.48 \\ \text { Dx,g cm-3 } & 1.575 \\ \mathrm{Z} & 4\end{array}$

$\mathrm{C}-\mathrm{C}=0.0088 \mathrm{~A}$

$b=26.9440(2)$

beta $=112.980(1)$
Wavelength $=1.54184$

$\mathrm{c}=10.2874(1)$

gamma $=90$
Reported

2363.69(4)

P 1211

$\mathrm{P} 2 \mathrm{yb}$

2(C7 H6 F3 N O2 S),

2(C13 H12 F3 N O4 S)

C38 H36 F13 N6 O11 S4

1127.97

1.585

2 


\begin{tabular}{|c|c|c|}
\hline $\mathrm{Mu}(\mathrm{mm}-1)$ & 2.861 & 2.892 \\
\hline F000 & 1144.0 & 1150.0 \\
\hline F000' & 1151.17 & \\
\hline h,k,lmax & $11,33,12$ & $11,33,12$ \\
\hline Nref & 9505[ 4859] & 9336 \\
\hline Tmin,Tmax & $0.525,0.561$ & $0.101,1.000$ \\
\hline Tmin' & 0.400 & \\
\hline \multicolumn{3}{|c|}{ Correction method $=\#$ Reported T Limits: Tmin $=0.101$ Tmax $=1.000$ AbsCorr $=$ MULTI-SCAN } \\
\hline Data completeness $=1.92 / 0.98$ & \multicolumn{2}{|c|}{$\operatorname{Theta}(\max )=73.314$} \\
\hline $\mathrm{R}($ reflections $)=0.0655(9156)$ & \multicolumn{2}{|c|}{$w R 2($ reflections $)=0.1750(9336)$} \\
\hline$S=1.078$ & Npar $=6$ & \\
\hline
\end{tabular}

Compound 11a (The ellipsoid contour percent probability level is 50\%.)

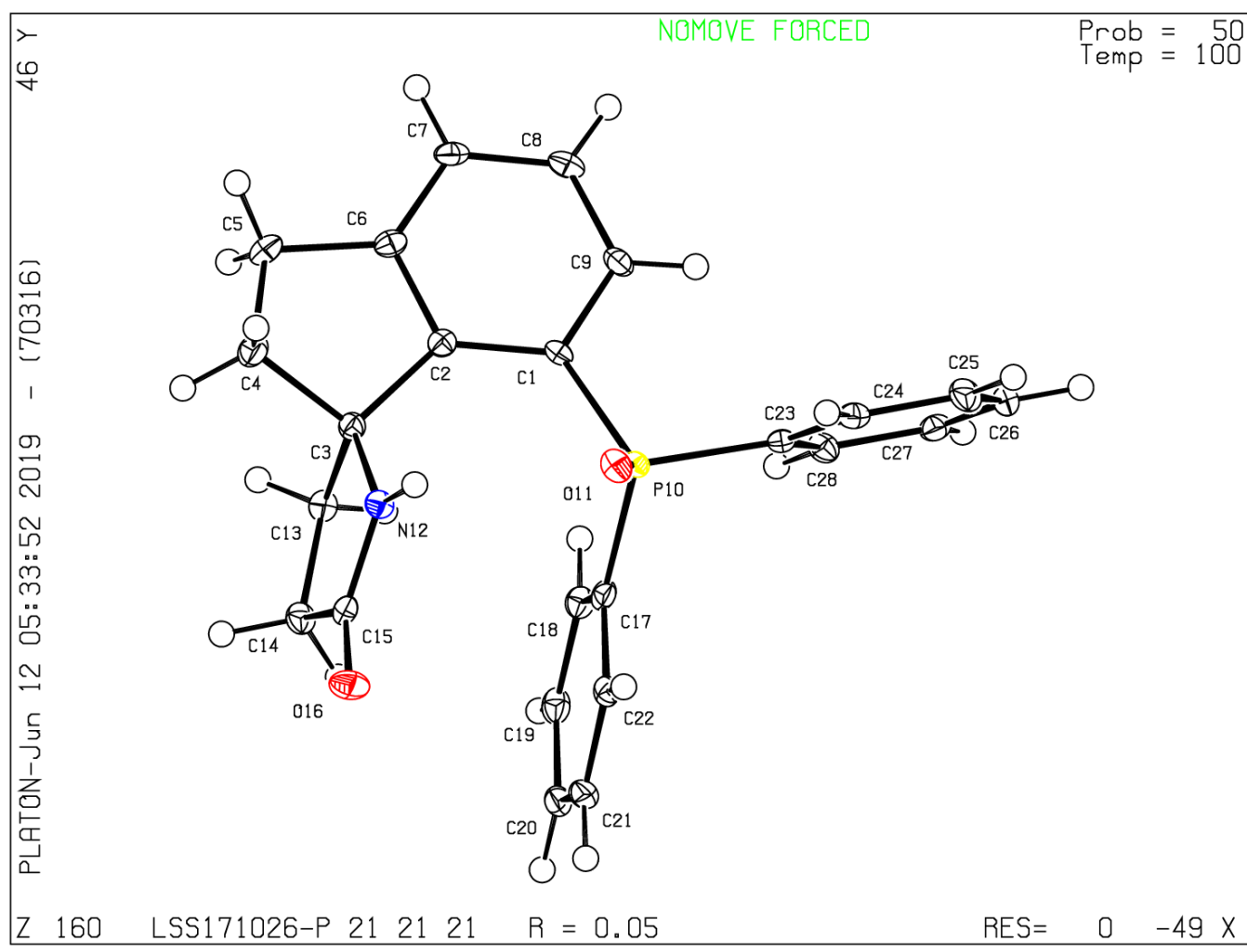

$\begin{array}{llll}\text { Bond precision: } & \mathrm{C}-\mathrm{C}= & & \\ \text { Cell: } & \mathrm{a}=8.3691(2) & \mathrm{b}=9.9458(2) & \mathrm{A}=22.7743(5) \\ & \text { alpha }=90 & \text { beta }=90 & \text { gamma }=90\end{array}$

Temperature: $100 \mathrm{~K}$

$\begin{array}{lll} & \text { Calculated } & \text { Reported } \\ \text { Volume } & 1895.67(7) & 1895.67(7) \\ \text { Space group } & \text { P } 212121 & \text { P } 212121\end{array}$




$\begin{array}{lll}\text { Hall group } & \text { P 2ac 2ab } & \text { P 2ac 2ab } \\ \text { Moiety formula } & \text { C24 H22 N O2 P } & \text { C24 H22 N O2 P } \\ \text { Sum formula } & \text { C24 H22 N O2 P } & \text { C24 H22 N O2 P } \\ \text { Mr } & 387.40 & 387.42 \\ \text { Dx,g cm-3 } & 1.357 & 1.357 \\ \text { Z } & 4 & 4 \\ \text { Mu (mm-1) } & 1.442 & 1.442 \\ \text { F000 } & 816.0 & 819.5 \\ \text { F000' } & 819.33 & 10,12,28 \\ \text { h,k,lmax } & 10,12,28 & 3755 \\ \text { Nref } & 3818[2201] & 0.715,1.000 \\ \text { Tmin,Tmax } & 0.625,0.749 & \\ \text { Tmin' } & 0.535 & \text { Theta(max)=73.350 } \\ \text { Correction method= \# Reported T Limits: Tmin=0.715 Tmax=1.000 AbsCorr = MULTI-SCAN } \\ \text { Data completeness=1.71/0.98 } \\ \text { R(reflections)=0.0498( 3371) } \\ \text { S = 1.007 }\end{array}$

Compound 11d (The ellipsoid contour percent probability level is 50\%.)

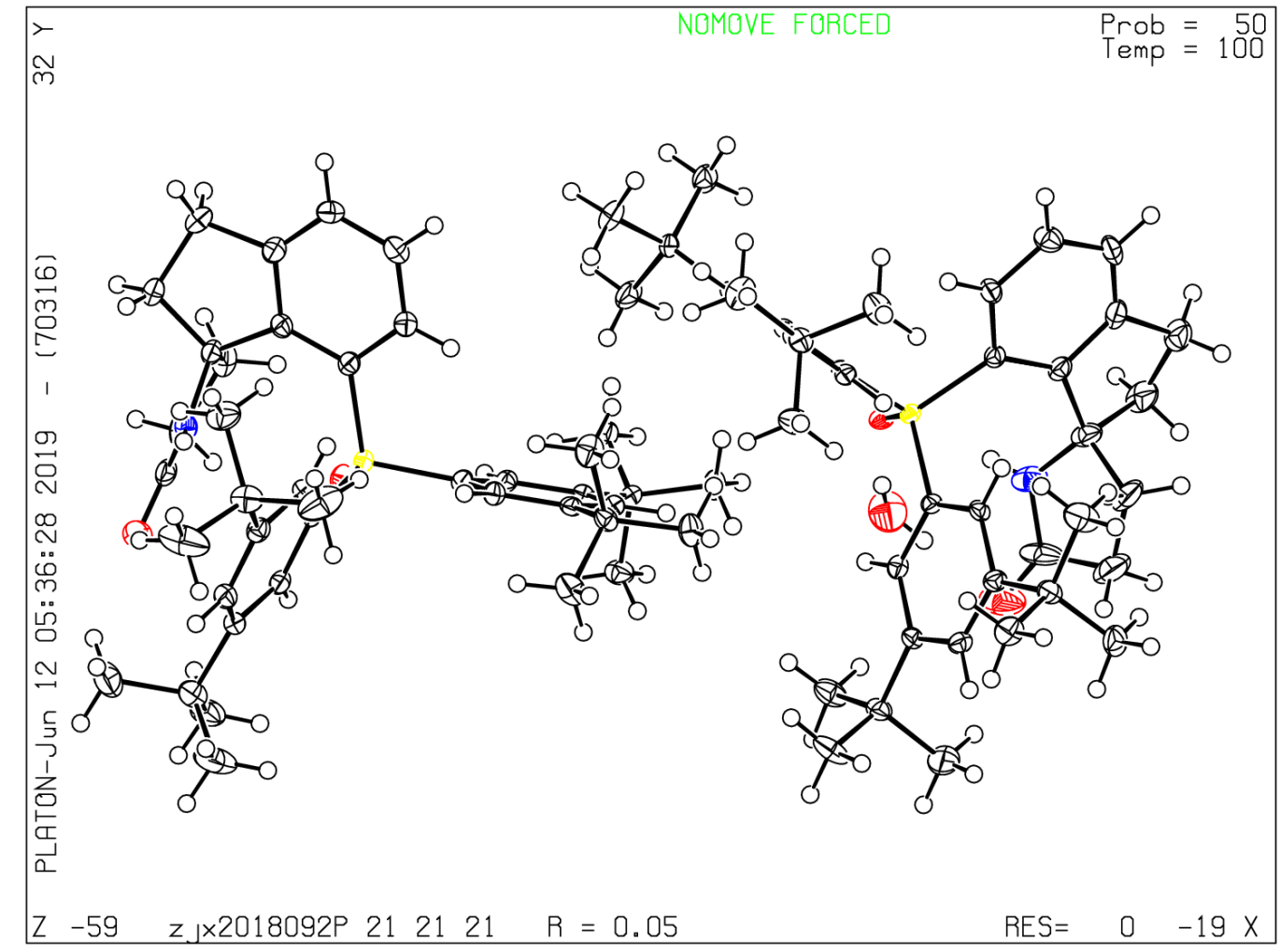




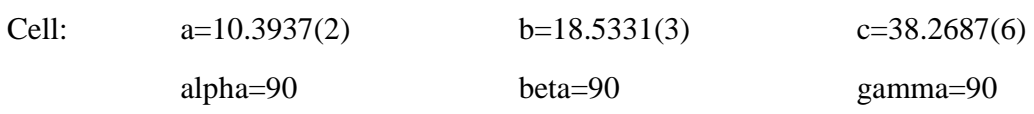

Temperature: $100 \mathrm{~K}$

\begin{tabular}{|c|c|c|}
\hline & Calculated & Reported \\
\hline Volume & $7371.6(2)$ & $7371.6(2)$ \\
\hline Space group & P 212121 & P 212121 \\
\hline Hall group & P 2ac 2ab & P $2 a c 2 a b$ \\
\hline Moiety formula & 2(C40 H54 N O2 P), H2 O & 2(C40 H54 N O2 P), H2 O \\
\hline Sum formula & C80 H110 N2 O5 P2 & C80 H110 N2 O5 P2 \\
\hline $\mathrm{Mr}$ & 1241.64 & 1241.63 \\
\hline Dx,g cm-3 & 1.119 & 1.119 \\
\hline $\mathrm{Z}$ & 4 & 4 \\
\hline $\mathrm{Mu}(\mathrm{mm}-1)$ & 0.916 & 0.916 \\
\hline F000 & 2696.0 & 2696.0 \\
\hline F000' & 2705.06 & \\
\hline $\mathrm{h}, \mathrm{k}, \operatorname{lmax}$ & $12,22,47$ & $12,22,46$ \\
\hline Nref & $14508[8008]$ & 14040 \\
\hline Tmin,Tmax & $0.755,0.912$ & $0.814,1.000$ \\
\hline Tmin' & 0.660 & \\
\hline \multicolumn{3}{|c|}{ Correction method $=\#$ Reported T Limits: Tmin $=0.814$ Tmax=1.000 AbsCorr $=$ MULTI-SCAN } \\
\hline \multicolumn{2}{|c|}{ Data completeness $=1.75 / 0.97$} & \\
\hline \multicolumn{2}{|c|}{$\mathrm{R}($ reflections $)=0.0480(13276)$} & $(14040)$ \\
\hline$S=1.064$ & Npar $=829$ & \\
\hline
\end{tabular}

$N$-[(S,E)-1,3-Diphenylallyl]-(R)-phenylglycinol (The ellipsoid contour percent probability level is $50 \%$.) 


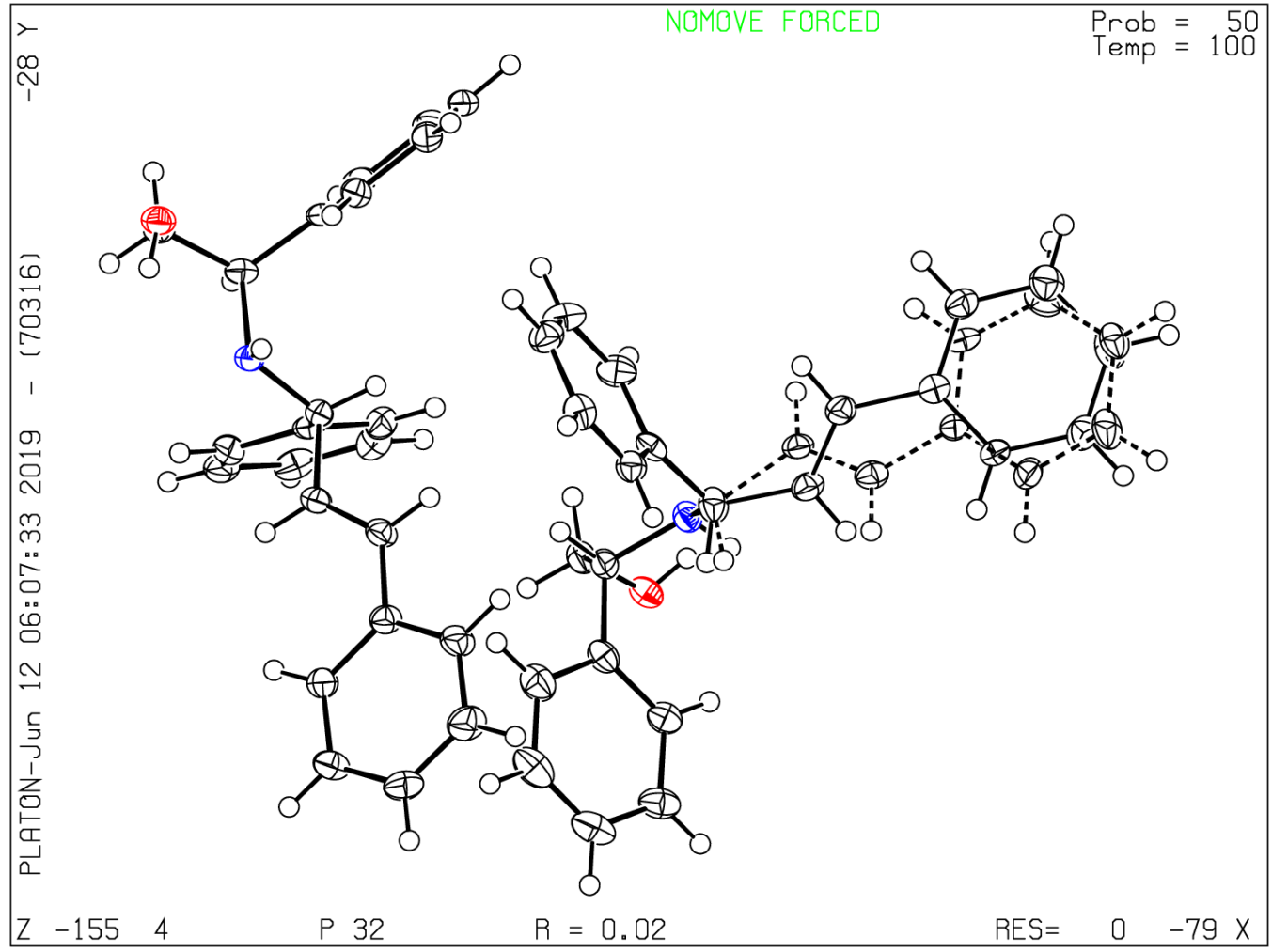

Bond precision:

$\mathrm{C}-\mathrm{C}=0.0037 \mathrm{~A}$

$b=10.2745(1)$

beta $=90$
Wavelength $=1.54178$

$c=30.2876(2)$

gamma $=120$

Temperature: $100 \mathrm{~K}$

Calculated

Volume

Space group

Hall group

Moiety formula

Sum formula

$\mathrm{Mr}$

Dx, $g$ cm-3

$\mathrm{Z}$

$\mathrm{Mu}(\mathrm{mm}-1)$

F000

F000'

h,k,lmax

Nref

Tmin,Tmax

Tmin'
2768.96(7)

P 32

P 32

C23 H23 N O

C23 H23 N O

329.42

1.185

6

0.555

1056.0

1058.83

$12,12,36$

6760[ 3380]

$0.819,0.847$

0.801
Reported

2768.96(6)

P 32

P 32

C23 H23 N O

C23 H23 N O

329.42

1.185

6

0.555

1056.0

$12,12,36$

6768 
Correction method $=$ Not given

Data completeness $=2.00 / 1.00$

$\operatorname{Theta}(\max )=68.240$

$\mathrm{R}($ reflections $)=0.0234(6753)$

$\mathrm{wR} 2$ (reflections) $=0.0595(6768)$

$\mathrm{S}=1.023$

Npar $=527$

$\left[\mathbf{P d}(\mathbf{I I})\left(\boldsymbol{\eta}^{3}-\mathbf{P h C H C H C H P h}\right)(\mathbf{1 d})\right] \mathbf{P F}_{\mathbf{6}}$ (The ellipsoid contour percent probability level is $50 \%$.)

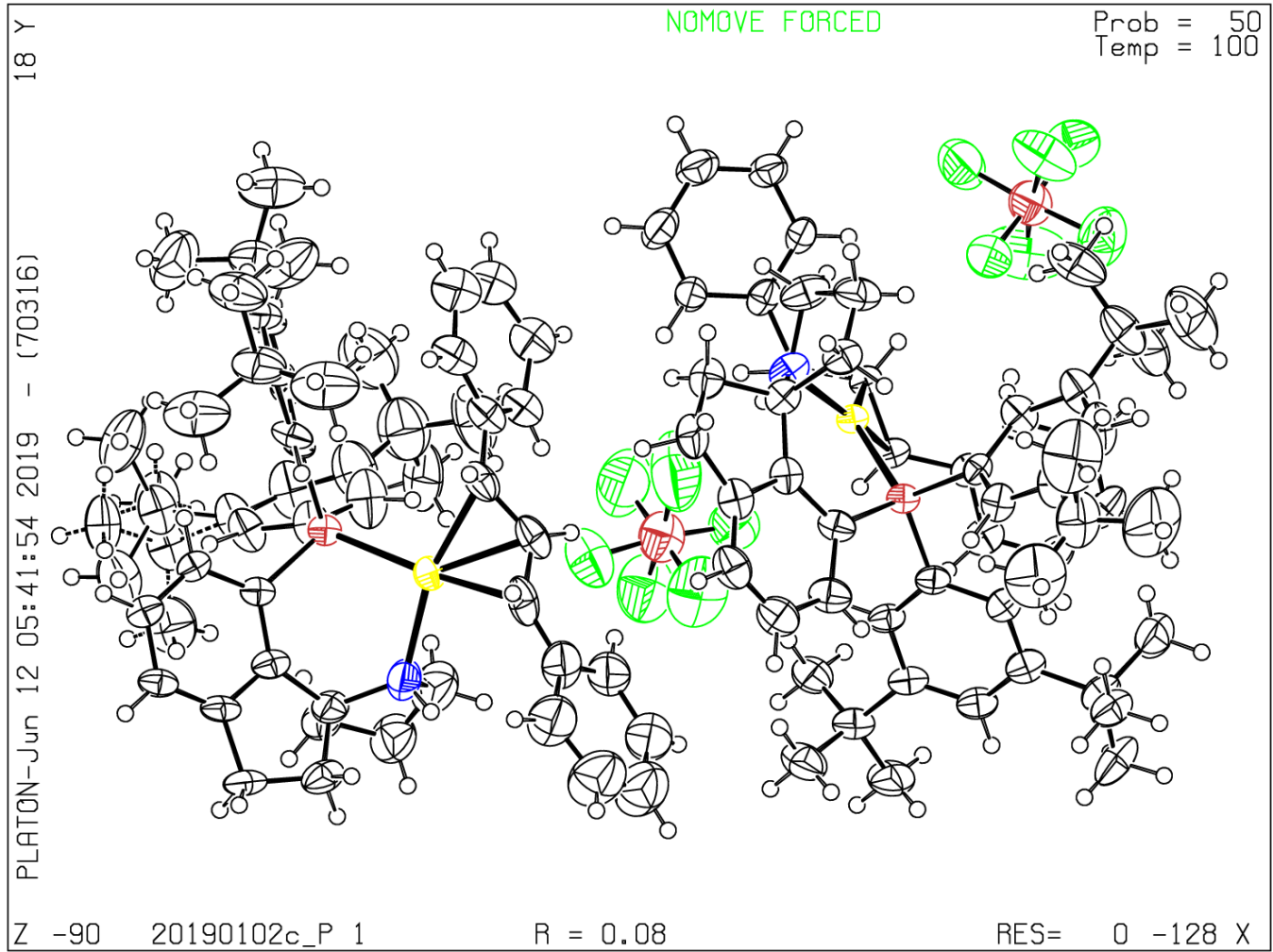

Bond precision:

Cell:

$$
\begin{aligned}
& a=13.3837(5) \\
& \text { alpha }=88.693(2)
\end{aligned}
$$

$\mathrm{C}-\mathrm{C}=0.0220 \mathrm{~A}$

$\mathrm{b}=13.4989(4)$

beta $=74.851(3)$
Wavelength $=0.71073$

$c=17.7272(6)$

gamma $=74.191(3)$

Temperature: $100 \mathrm{~K}$

\section{Calculated}

Volume

Space group

Hall group

Moiety formula

Sum formula

$\mathrm{Mr}$

Dx, $g$ cm-3

Z

$\mathrm{Mu}(\mathrm{mm}-1)$
2970.42(19)

P 1

P 1

C55 H69 N P Pd, F6 P

C55 H69 F6 N P2 Pd

1026.45

1.148

2

0.416
Reported

2970.42(18)

P 1

P 1

C55 H69 N P Pd, F6 P

C55 H69 F6 N P2 Pd

1026.45

1.148

2

0.416 
F000

1072.0

1072.0

F000

1070.88

h,k,lmax

$16,16,22$

$16,16,22$

Nref

24604[ 12302]

24352

Tmin,Tmax

$0.861,0.920$

$0.758,1.000$

Tmin'

0.747

Correction method $=$ \# Reported $\mathrm{T}$ Limits: $\mathrm{Tmin}=0.758 \mathrm{Tmax}=1.000$ AbsCorr $=$ MULTI-SCAN

Data completeness $=1.98 / 0.99$

Theta $(\max )=26.500$

$\mathrm{R}($ reflections $)=0.0809(18428)$

$\mathrm{wR} 2$ (reflections) $=0.2321(24352)$

$\mathrm{S}=1.050$

Npar $=1208$ 


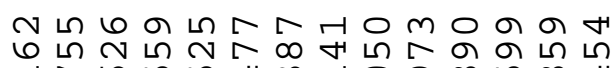

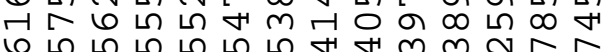
ón 0

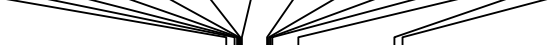<smiles>CCOC(=O)CCC(=O)/C=C/c1ccccc1</smiles>

구율유 舟开卉 $\dot{\nabla} \dot{+} \dot{\theta}$ I m MN N

0 $\infty \bullet$ NN 4iti
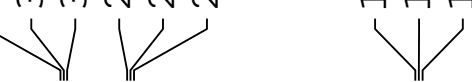

NAME PROCNO Date

Time

INSTRUM

PROBHD

PULPROG

TD

SOLVENT

DS

FWH

$A Q$

$\mathrm{RG}$

DW

DE

D1

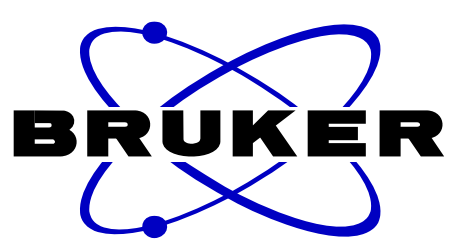

CWG160111-pure

20160112

17.08

$5 \mathrm{~mm}$ PABBO BB-

$\mathrm{zg} 30$

65536

$\mathrm{CDCl} 3$

16

$8223.685 \mathrm{~Hz}$

$0.125483 \mathrm{~Hz}$

$3.9846387 \mathrm{sec}$ 161

60.800 usec

6.50 usec

$289.2 \mathrm{~K}$

$1.00000000 \mathrm{sec}$
1

$=======$ CHANNEL

$\mathrm{NUC1}$

P1

PL1W

SF01

SI
WF
WDW

WDW

LB

GB

$P C$

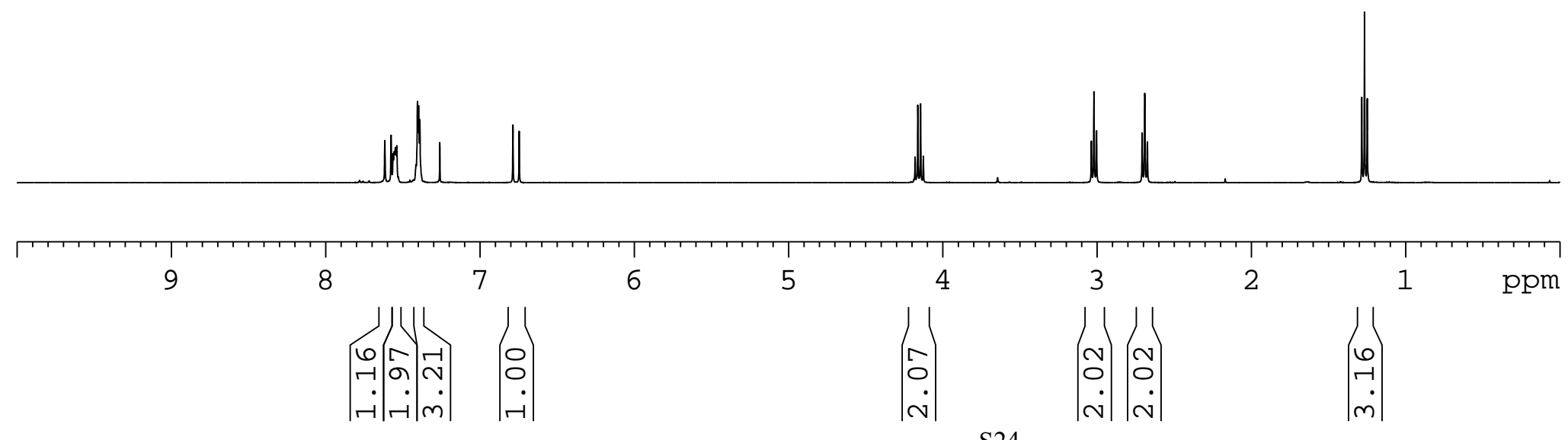

13.80 usec

$1.00 \mathrm{~dB}$

$13.18669796 \mathrm{~W}$

400.1724712 MHz

32768
400.1700033

$0033 \mathrm{MHZ}$

EM

$\odot .30 \mathrm{~Hz}$

1. $\odot$ 


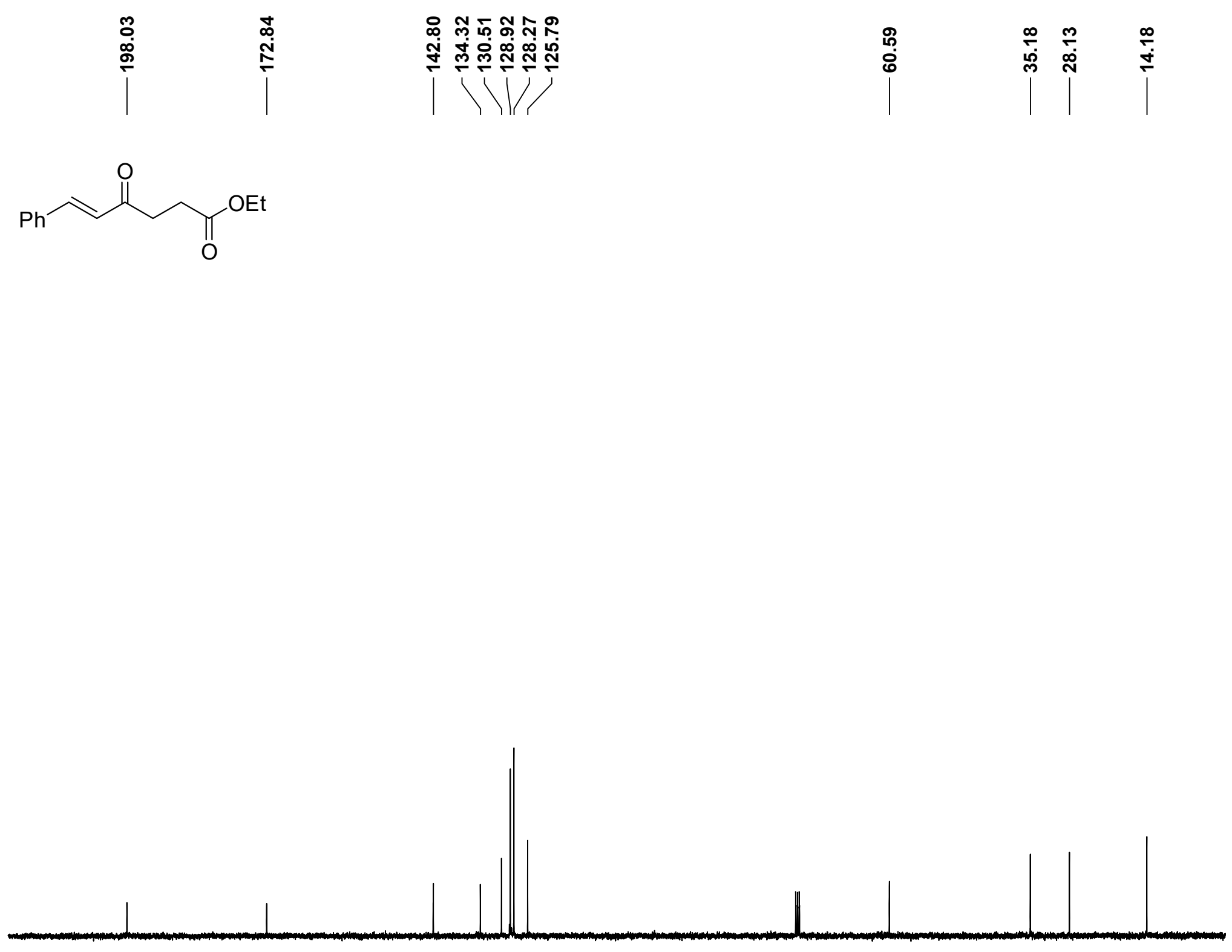

$210200 \quad 190$ 
<smiles>CCOC(=O)CCC(=O)CCc1ccccc1</smiles>

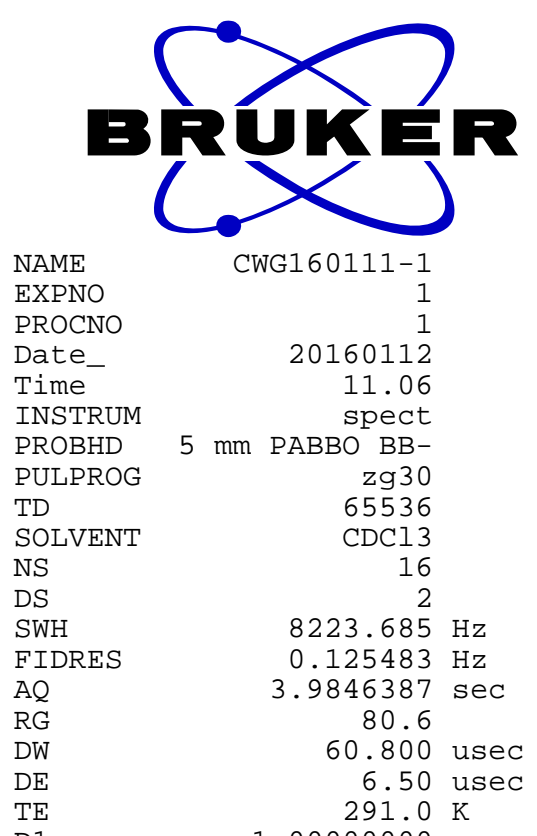

D1 TDO

$1.00000000 \mathrm{sec}$

==ニ= NUC1

PL1

PL1W

SF01

SF

WDW

SSB

GB

PC

BRUMER

CWG160111-1

60112 $23.685 \mathrm{~Hz}$ $125483 \mathrm{~Hz}$ 80.6

$=$ CHANNEL $=======$ $1 \mathrm{H}$ 13.80 use $13.18669796 \mathrm{~W}$ 400.1724712 MHZ 32768 $400.1700034 \mathrm{MHz}$

$\mathrm{EM}$
0
$30 \mathrm{~Hz}$
0
0

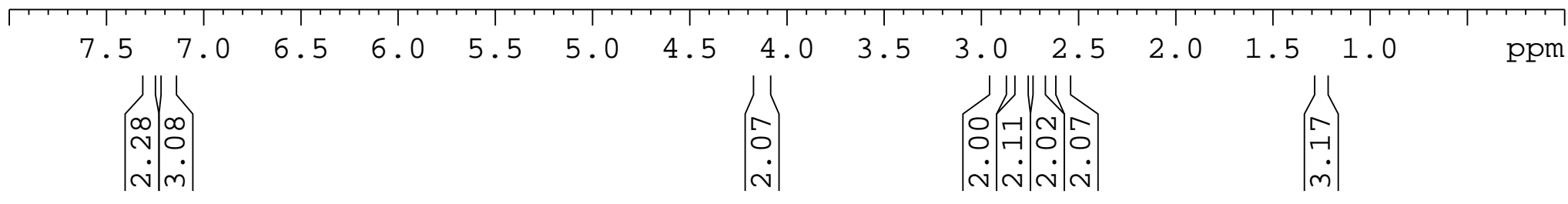




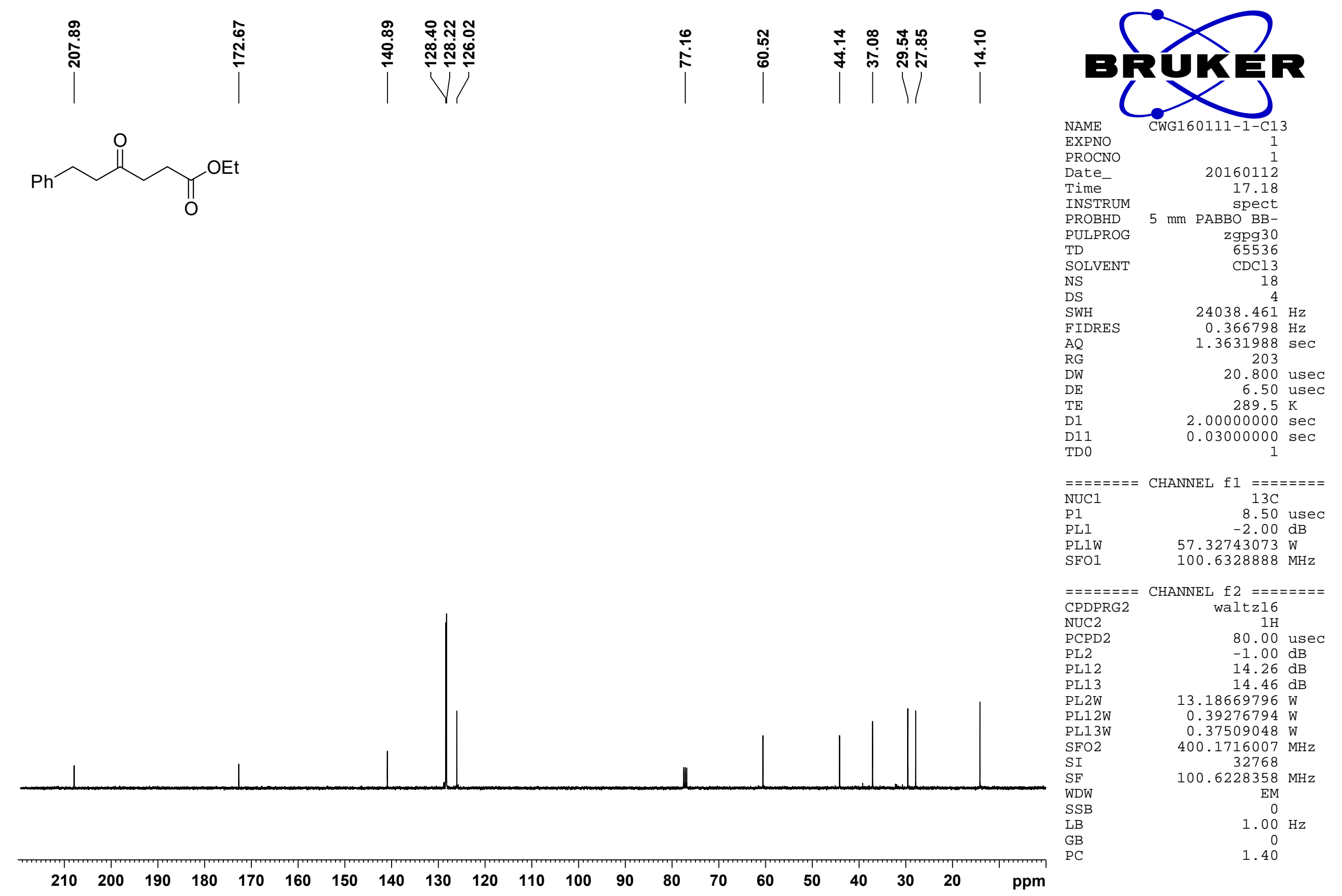


$m \infty$ 하 $m \infty$ in $m \sim \infty \sim ⿻ 上$ hrh h h h $\rightarrow \infty$

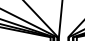<smiles>O=C(O)CCC(=O)CCc1ccccc1</smiles>

$2 \mathrm{a}$

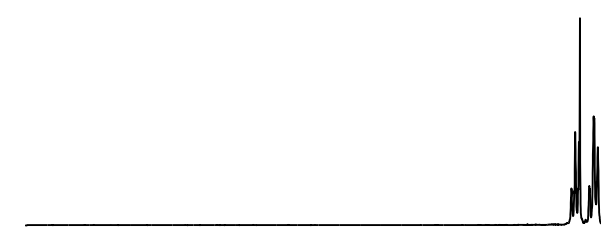

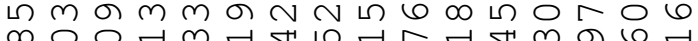

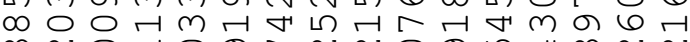

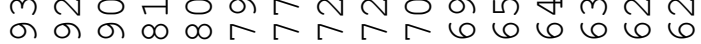
$\dot{\sim} \dot{\sim} \dot{\sim} \dot{\sim} \dot{\sim} \dot{\sim} \dot{\sim} \dot{\sim} \dot{\sim} \dot{\sim} \dot{\sim} \dot{\sim} \dot{\sim}$

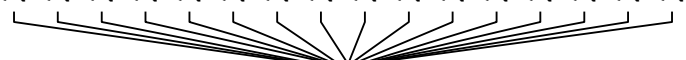
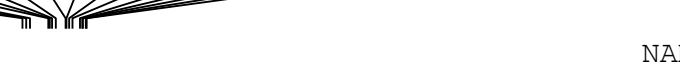

NAME

EXPNO

PROCNO

Date

Time

INSTRUM

PROBHD

PULPROG

TD

SOLVENT

NS

DS

FIDRES

RG

DW

$\mathrm{DE}$

D1

TDO

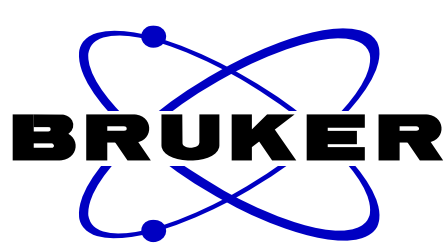

CWG160114-PURE

20160116

11.18

$5 \mathrm{~mm}$ РАBBO BB-

zg30
65536
$\mathrm{CDCl}$

2
$8223.685 \mathrm{~Hz}$

$0.125483 \mathrm{~Hz}$

$3.9846387 \mathrm{sec}$

203

60.800 usec

6.50 usec

$1.00000000 \mathrm{sec}$

$========$ CHANNEL f $1========$ NUC1

P1

PL1

SEO1

SI

SF

WDW

LB

GB

$===$

13.80 usec

$3.18669796 \mathrm{WT}$

$400.1724712 \mathrm{MHz}$

32768
$400.1700027 \mathrm{MHz}$

EM
0

$0.30 \mathrm{~Hz}$

1.00

Hill

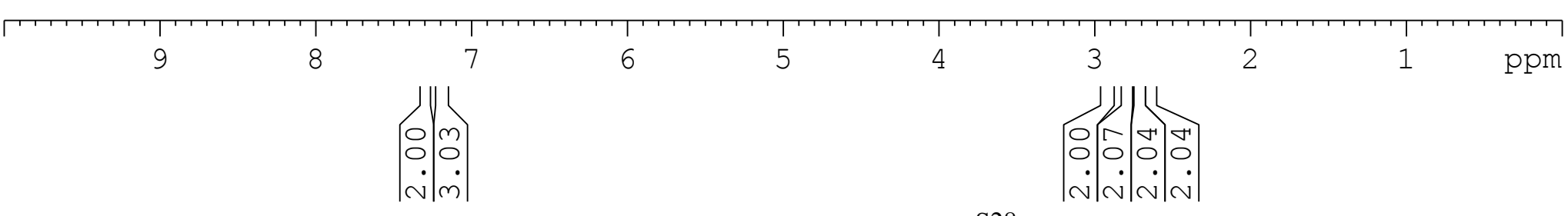




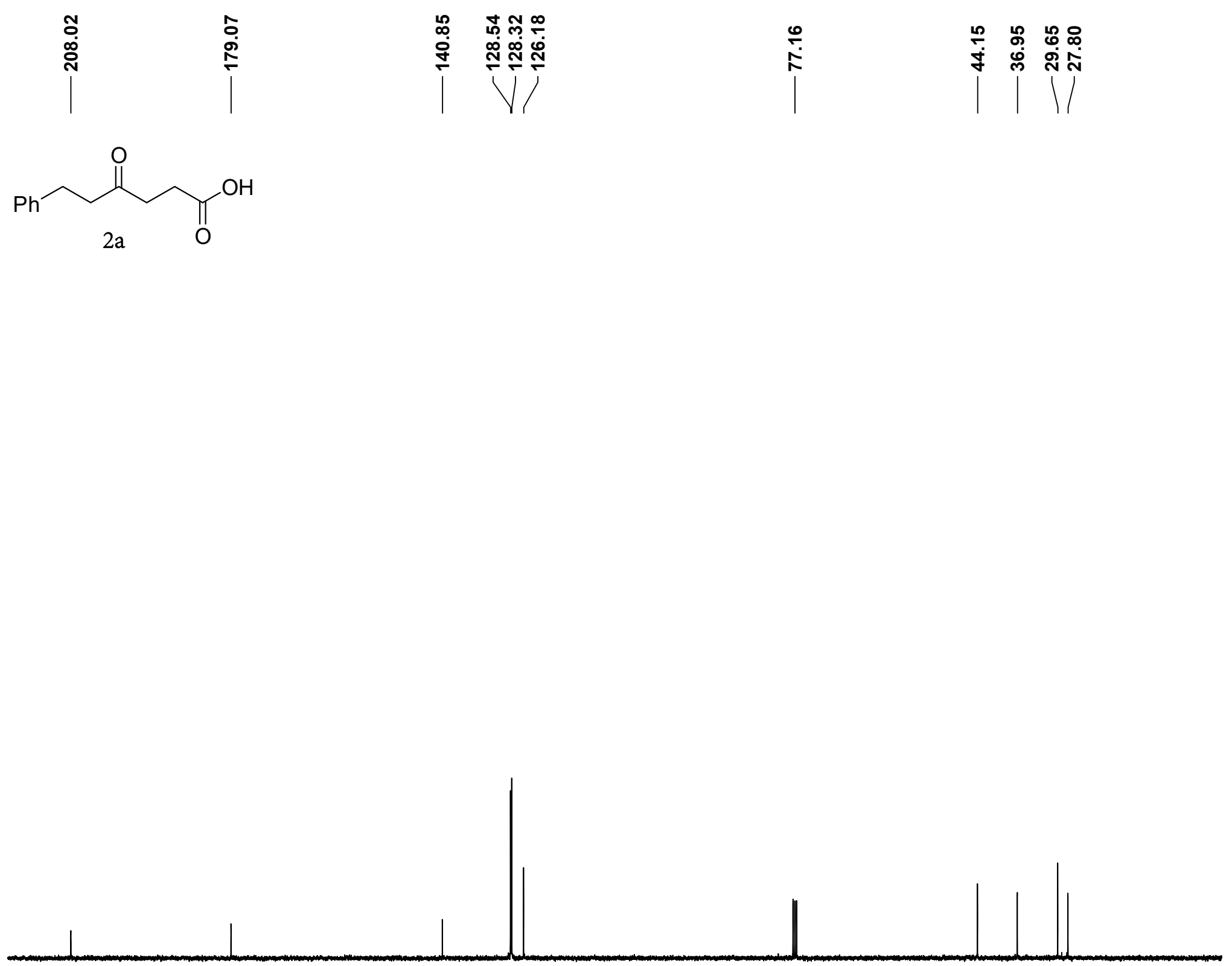

$===$
NUC1 


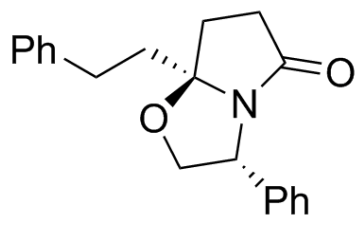

$3 a$ r

NAME

PROCNO

Date

Time

INSTRUM

PROBHD

PULPROG

TD

SOLVENT

NS

DS

SWH
FIDRES

$\mathrm{AQ}$
$\mathrm{RG}$

RG

DW

TE

D1

CWG160112

20160114

16.28

$5 \mathrm{~mm}$ PABBO BB-

zg30

65536

CDCl3

16
2

$8223.685 \mathrm{~Hz}$

$\odot .125483 \mathrm{~Hz}$

$3.9846387 \mathrm{sec}$ 203

60.800 usec

6.50 use

$1.00000000 \mathrm{sec}$

$====$

P1

PL1

SF01

SF

WDW

B

CHANNEL

1

13.80 usec
$-1.00 \mathrm{~dB}$
$13.18669796 \mathrm{~W}$
$400.1724712 \mathrm{MHz}$
$32768 \mathrm{MH}$
$400.1700033 \mathrm{MHz}$
$\mathrm{EM}$
$\odot$
$0.30 \mathrm{~Hz}$
$\odot$
1.00

1.00 
<smiles>O=C1CC[C@]2(CCc3ccccc3)OC[C@H](c3ccccc3)N12</smiles>

$\mathrm{Ph}$

$3 a$

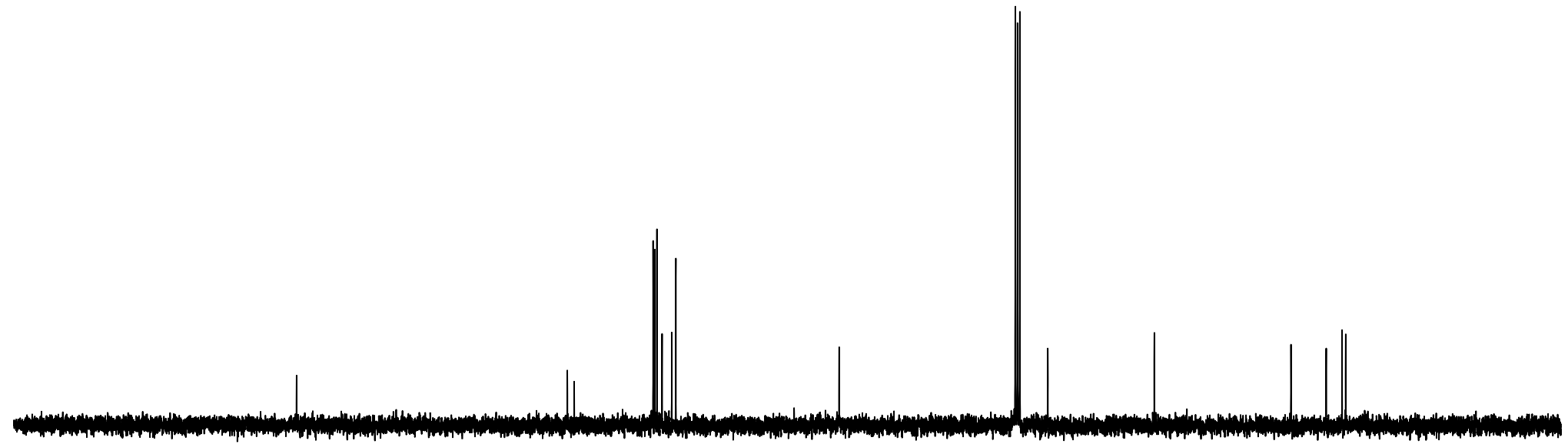

NUC1

$$
\begin{aligned}
& \text { PL1 } \\
& \text { PL1W } \\
& \text { SF01 }
\end{aligned}
$$

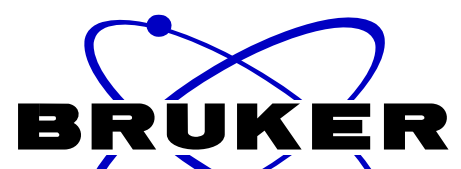

NAME

EXPNO

Date

$\mathrm{FLF}_{\text {FL } 16216-3-\mathrm{C}_{13}}$

1

(1)

PROBHD

PULPROG

TD

NS

SWH

FIDRES

AQ
RG
DW

DE

D1

D11

20160930

16.22

$5 \mathrm{~mm}$ РАВBO BВ-

zgpg 30

gpg30
65536

$\mathrm{CDCl3}$

4
24038.461

$24038.461 \mathrm{~Hz}$
$0.366798 \mathrm{~Hz}$

$1.3631988 \mathrm{sec}$ 203

20.800 usec

6.50 usec $299.5 \mathrm{~K}$

$2.00000000 \mathrm{sec}$ $0.03000000 \mathrm{sec}$

$==$ 1

\section{$=======$ CHANNEL}

f2 =-=-=-=

CPDPRG2

NCC2

-1
-12

PL12

PL2W

PL2W

PL13W

PL13W

SI

SF

WDW

LB

LB
GB
$P C$

$==$ CHANNEL $\mathrm{f} 1 \mathrm{=}=====$ 8.50 use $2.0 \odot \mathrm{dB}$ $57.32743073 \mathrm{~W}$

$100.6328888 \mathrm{MHz}$

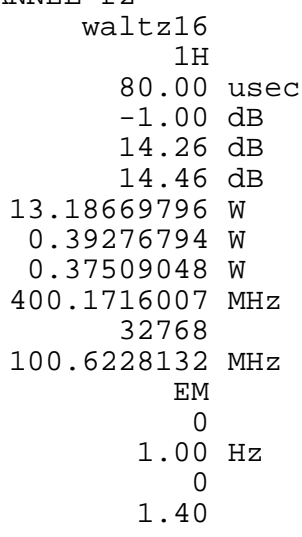


거요에

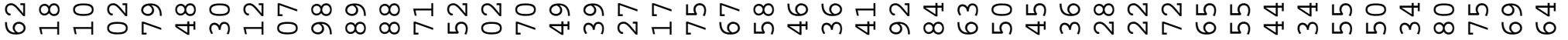
N NָN

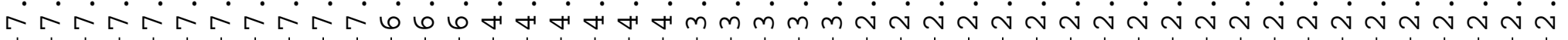
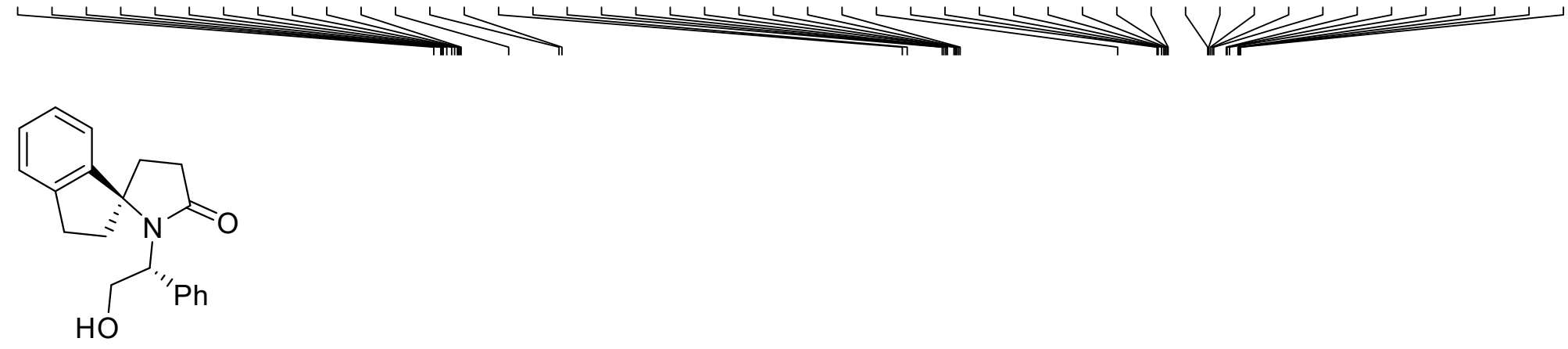

$4 \mathrm{a}$
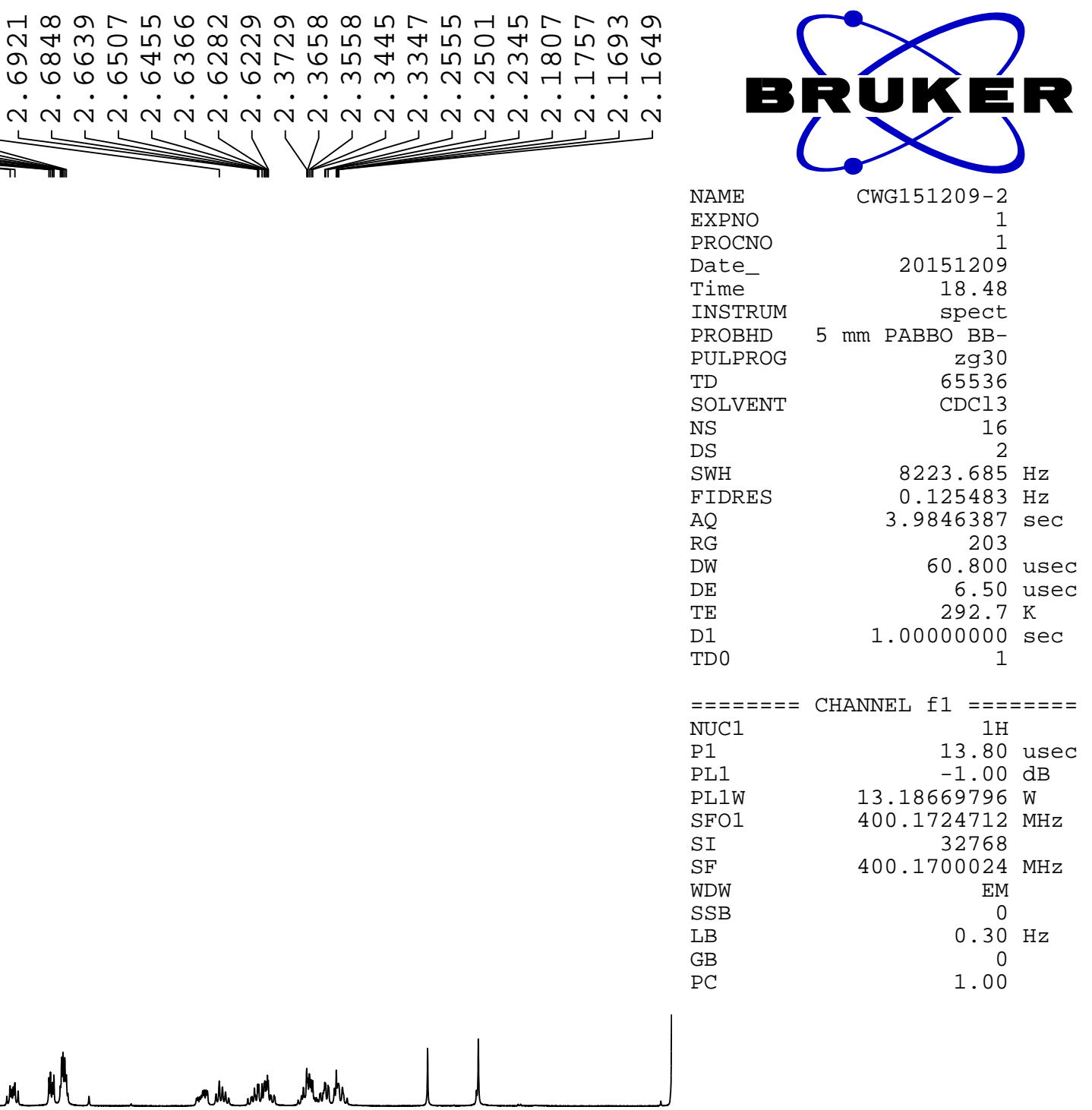

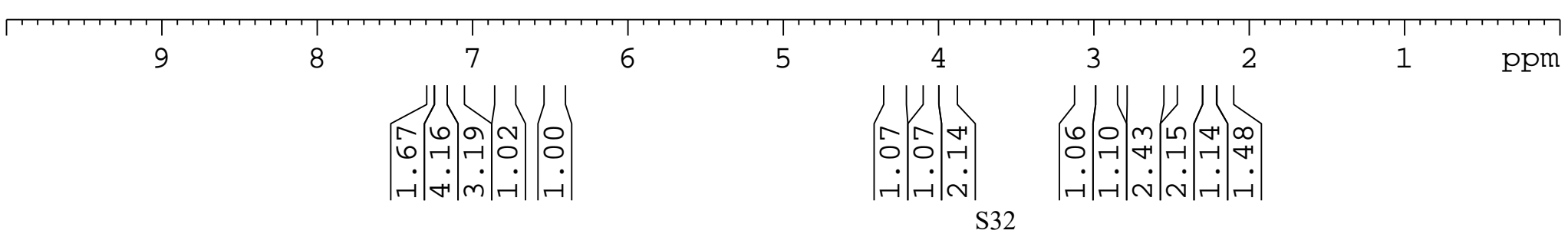




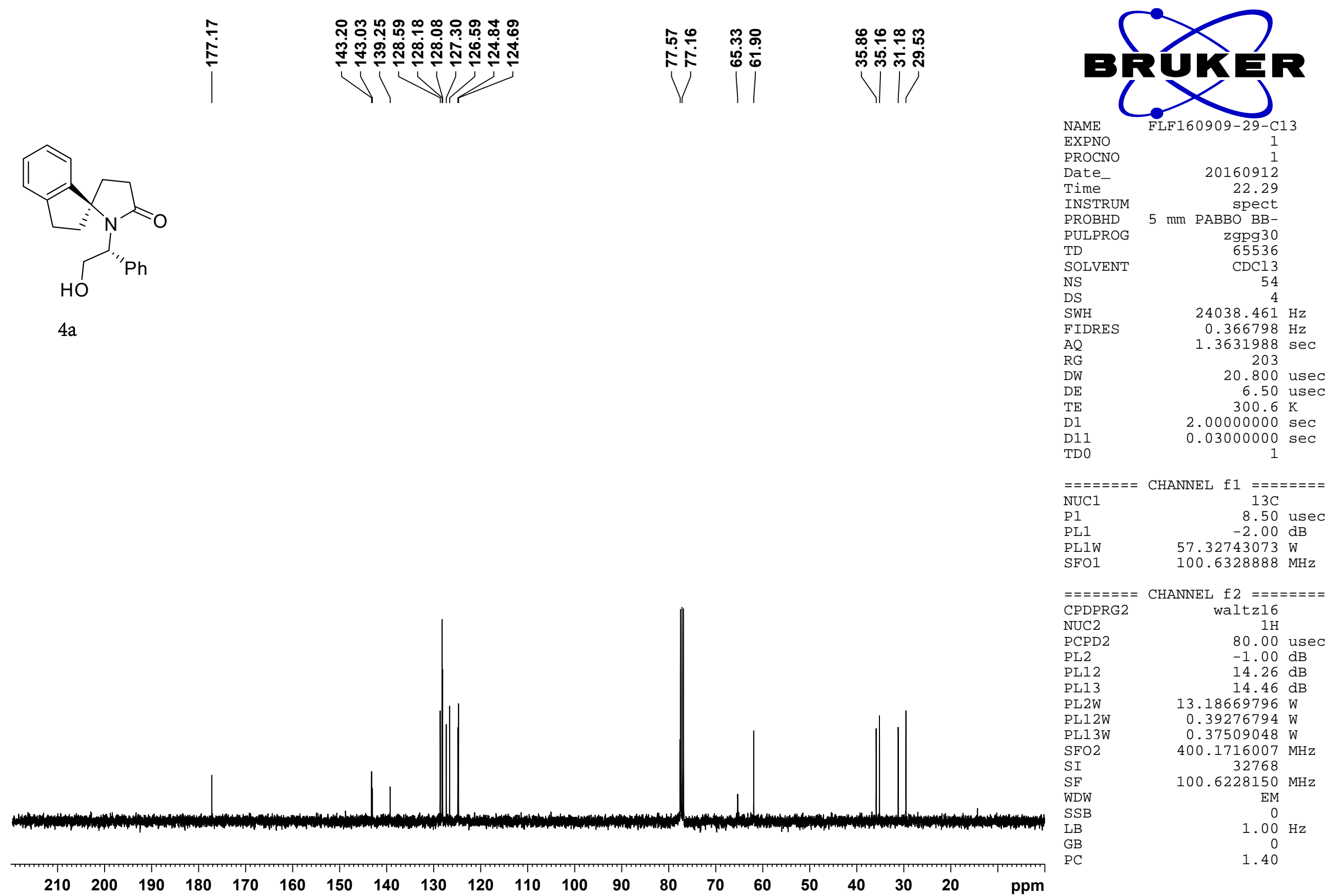




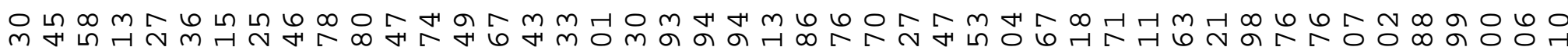

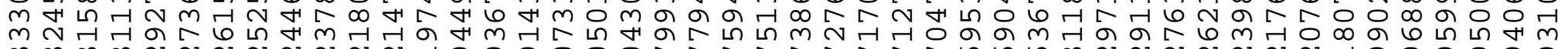

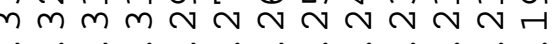

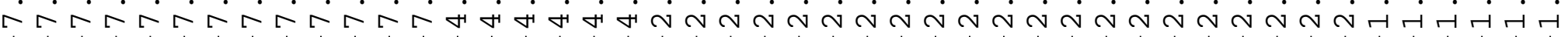
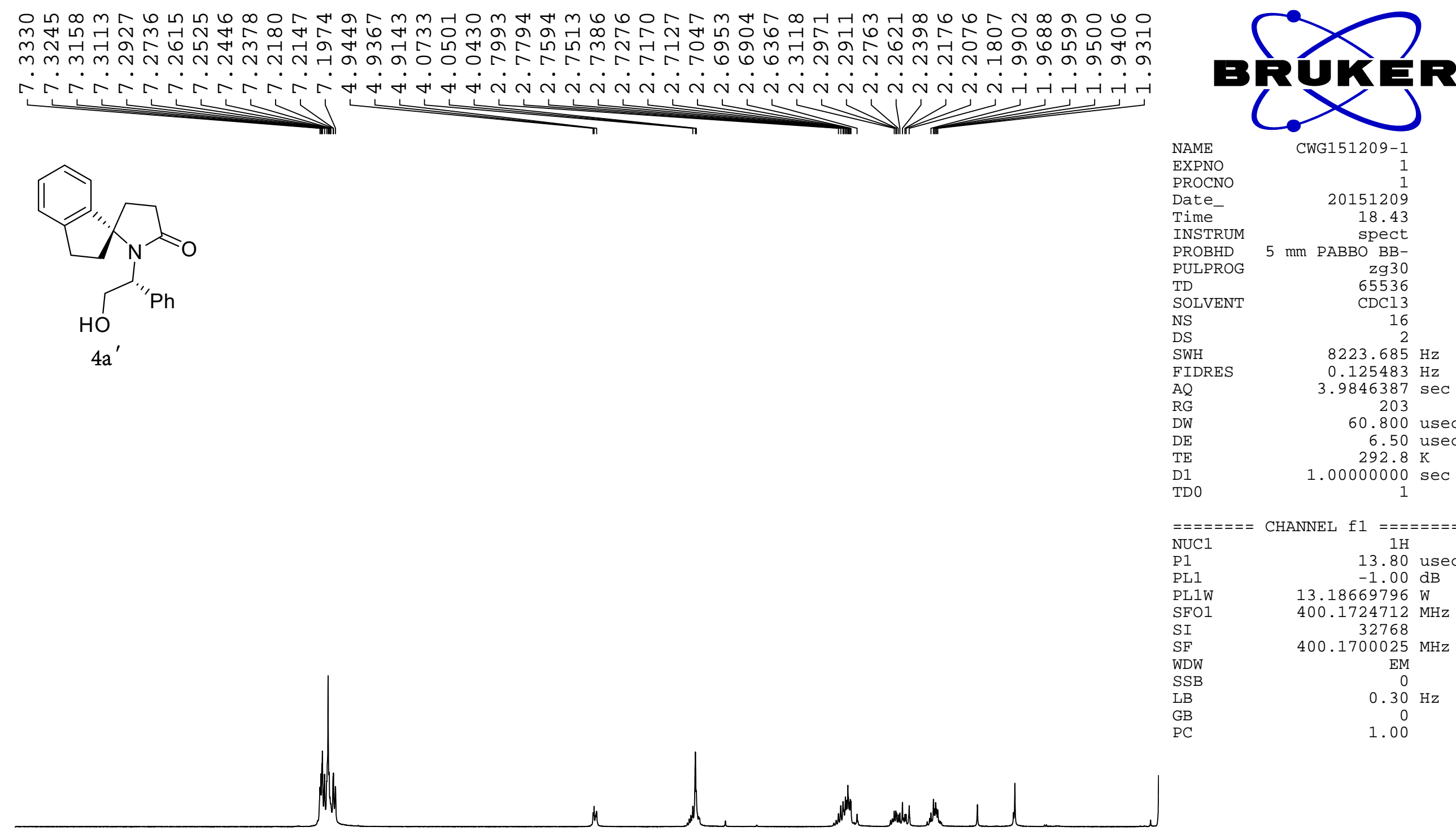

NAME

EXPNO

PROCNO

Date-

TimSTRUM

PROBHD

PULPROG

TD

SOLVENT

NS

DS

SWH
FIDRES

$A Q$
$R G$

$\mathrm{AQ}$
$\mathrm{RG}$
$\mathrm{DW}$
$\mathrm{DE}$

TE

D1

CWG151209-1

20151209

$\begin{array}{r}18.43 \\ \hline\end{array}$

$5 \mathrm{~mm}$ PABBO BB-

zg30
65536

CDCl3

16
2

$8223.685 \mathrm{~Hz}$

$0.125483 \mathrm{~Hz}$

$3.9846387 \mathrm{sec}$ 203

60.800 usec

6.50 use

$1.00000000 \mathrm{sec}$

$=======$ CHANNEL

NUC1

P1

PL1

SF01

SI

SF

WDW

SSB

$B$

GB

1

CHANNEL 1 =======

13.80 usec

$-1.00 \mathrm{~dB}$
$13.18669796 \mathrm{~W}$

$400.1724712 \mathrm{MHz}$

32768

$400.1700025 \mathrm{MHz}$

EM

$\odot .30 \mathrm{~Hz}$

$1.0 \odot$

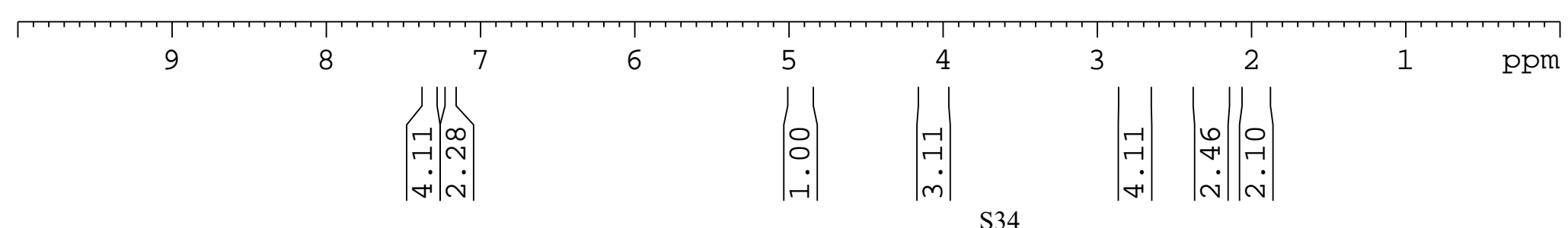




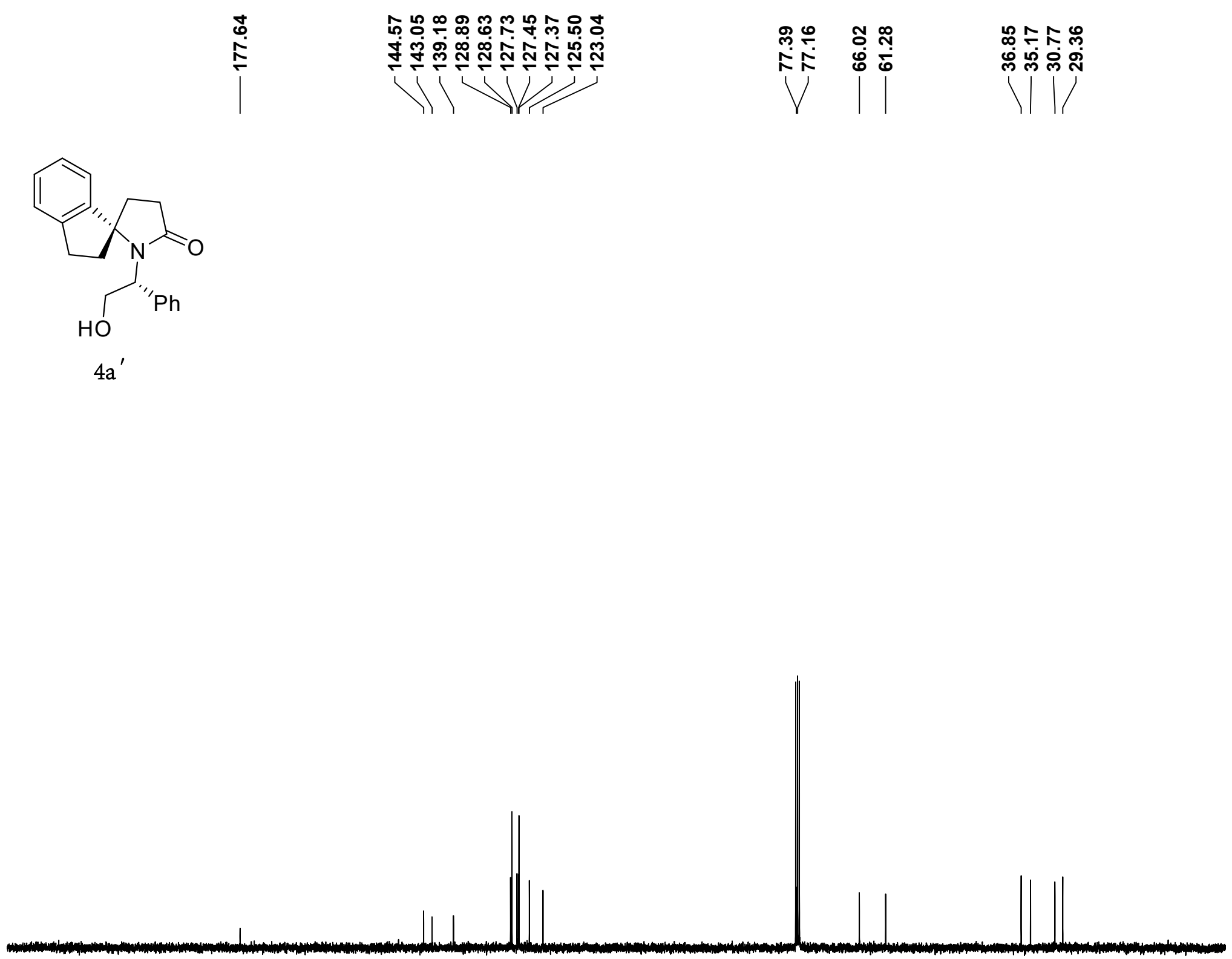
Bर $\underbrace{}_{\text {FLF160909-16-C13 }}$ NAME $\quad$ FLF160909-16-C13 EXPNO
PROCNO
Date
20160912
23.08
INSTRUM
PROBHD
$5 \mathrm{~mm}$ РАВBO BB-
Zgpg30
TD 65536
SOLVENT
CDCl3
DS
SWH
FIDRES
$A Q$
RG
DW
DE
D1
D11
86
4
$24038.461 \mathrm{~Hz}$
$0.366798 \mathrm{~Hz}$
$1.3631988 \mathrm{sec}$ 203
20.800 usec
6.50 usec $300.3 \mathrm{~K}$
$2.00000000 \mathrm{sec}$
$0.03000000 \mathrm{sec}$

$==$

NUC1

P1

PL1

$$
\text { SF01 }
$$

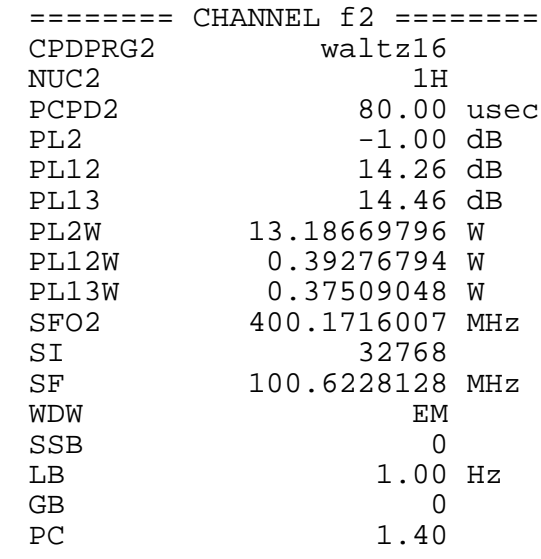




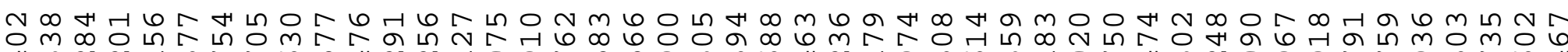

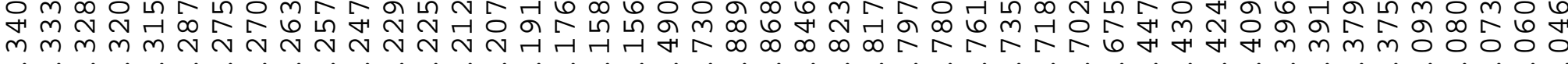

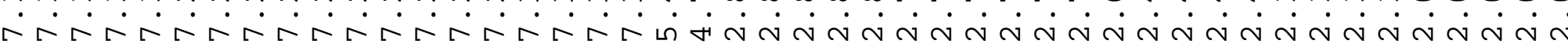
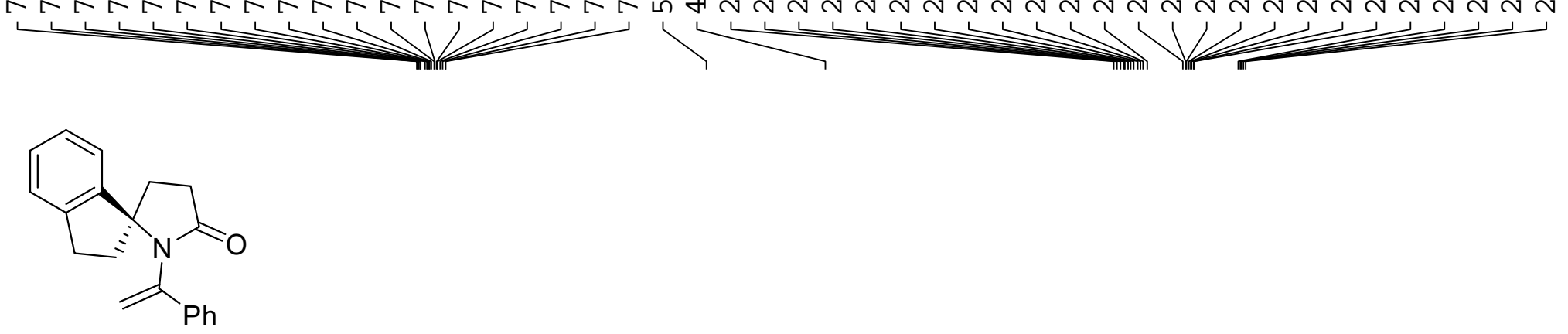

$5 a$

$\begin{array}{lr} & \\ & \end{array}$
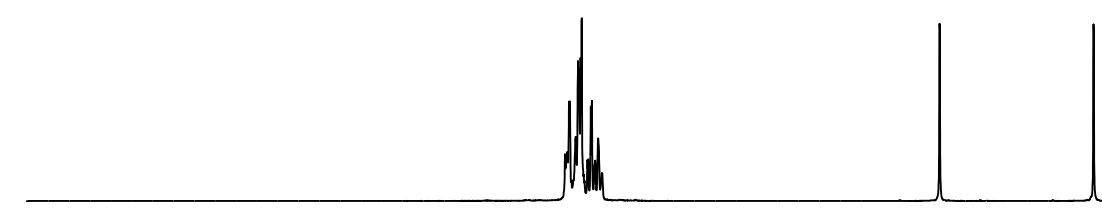

1.00

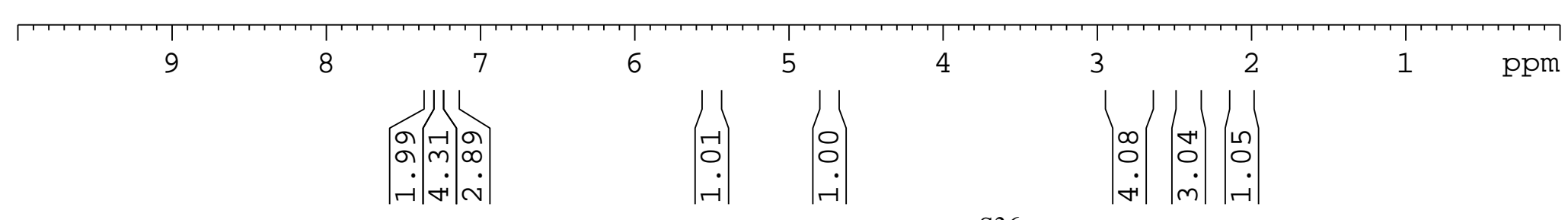




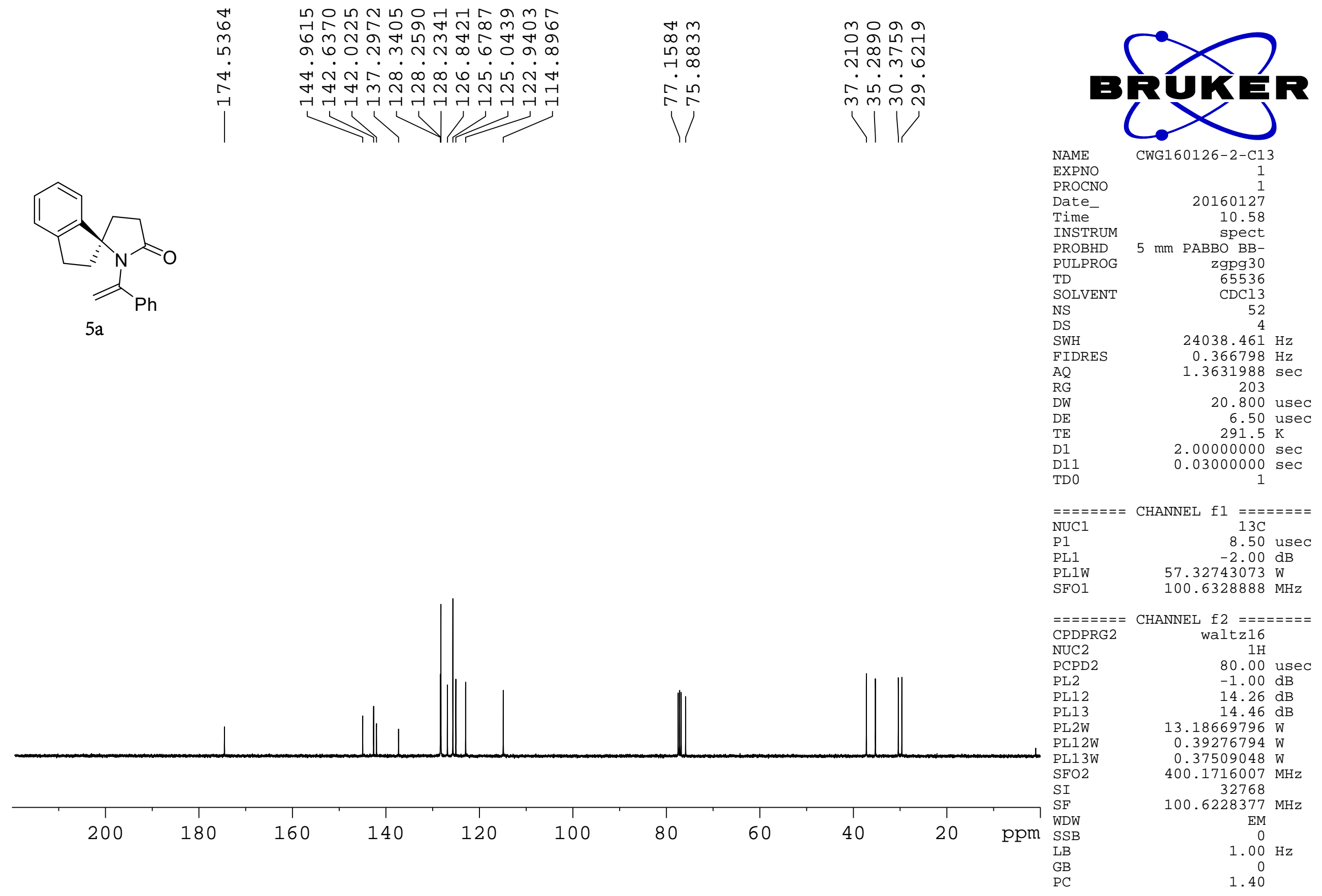




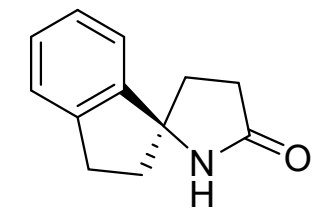

$6 a$
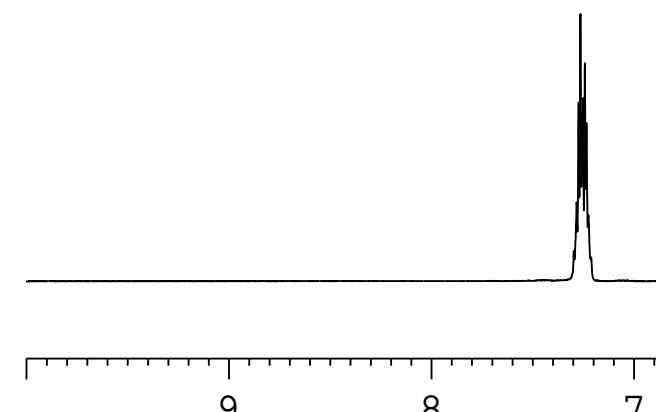

9

8

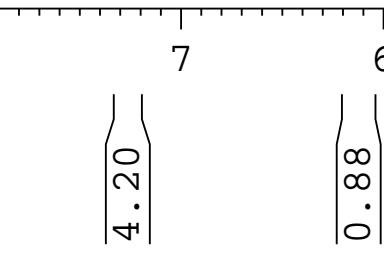
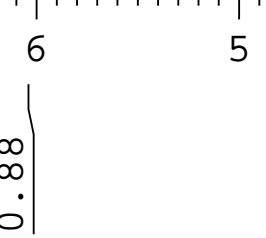

5

$\infty$

๑ं।
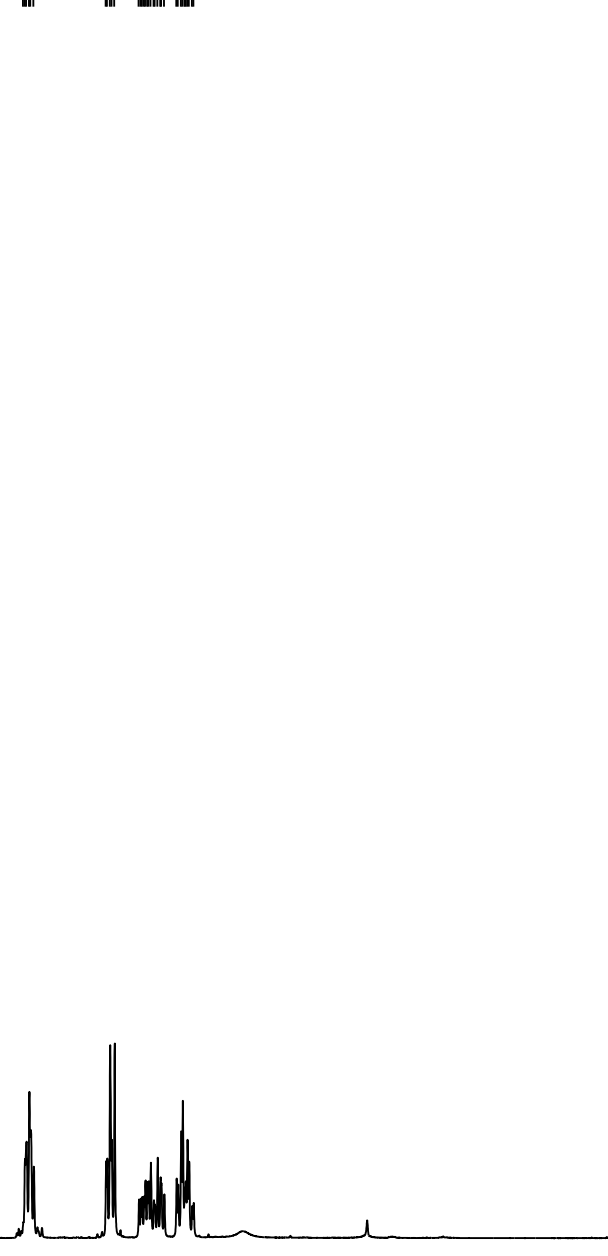

4

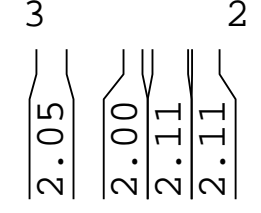

\section{NAME}

EXPNO

PROCNO

Date

INSTRUM

PROBHD

PULPROG

TD

SOLVENT

NS

SWH

FIDRES

$\mathrm{AQ}$
$\mathrm{RG}$
$\mathrm{DW}$

DE

TE

D1 0

Bivutar

$\underbrace{}_{F L F 160919-2-10}$

20160921

8.04

$5 \mathrm{~mm}$ РАBBO BB-

zg30

65536

$\mathrm{CDCl3}$

16
2

$8223.685 \mathrm{~Hz}$

$0.125483 \mathrm{~Hz}$

$3.9846387 \mathrm{sec}$

203

60.800 usec
6.50 usec

$298.1 \mathrm{~K}$

1. $00000000 \mathrm{sec}$

$====$

P1

PL1

SF01

SI

SI
SF
WDW

WDW

SSB

LB

GB

CHANNEL

$======$

13. 80 usec

$1.00 \mathrm{~dB}$

$13.18669796 \mathrm{~W}$

$400.1724712 \mathrm{MHz}$

32768

40๑. $1700 \odot 21 \mathrm{MHZ}$

EM

$0.30 \mathrm{~Hz}$

$1.0 \odot$ 


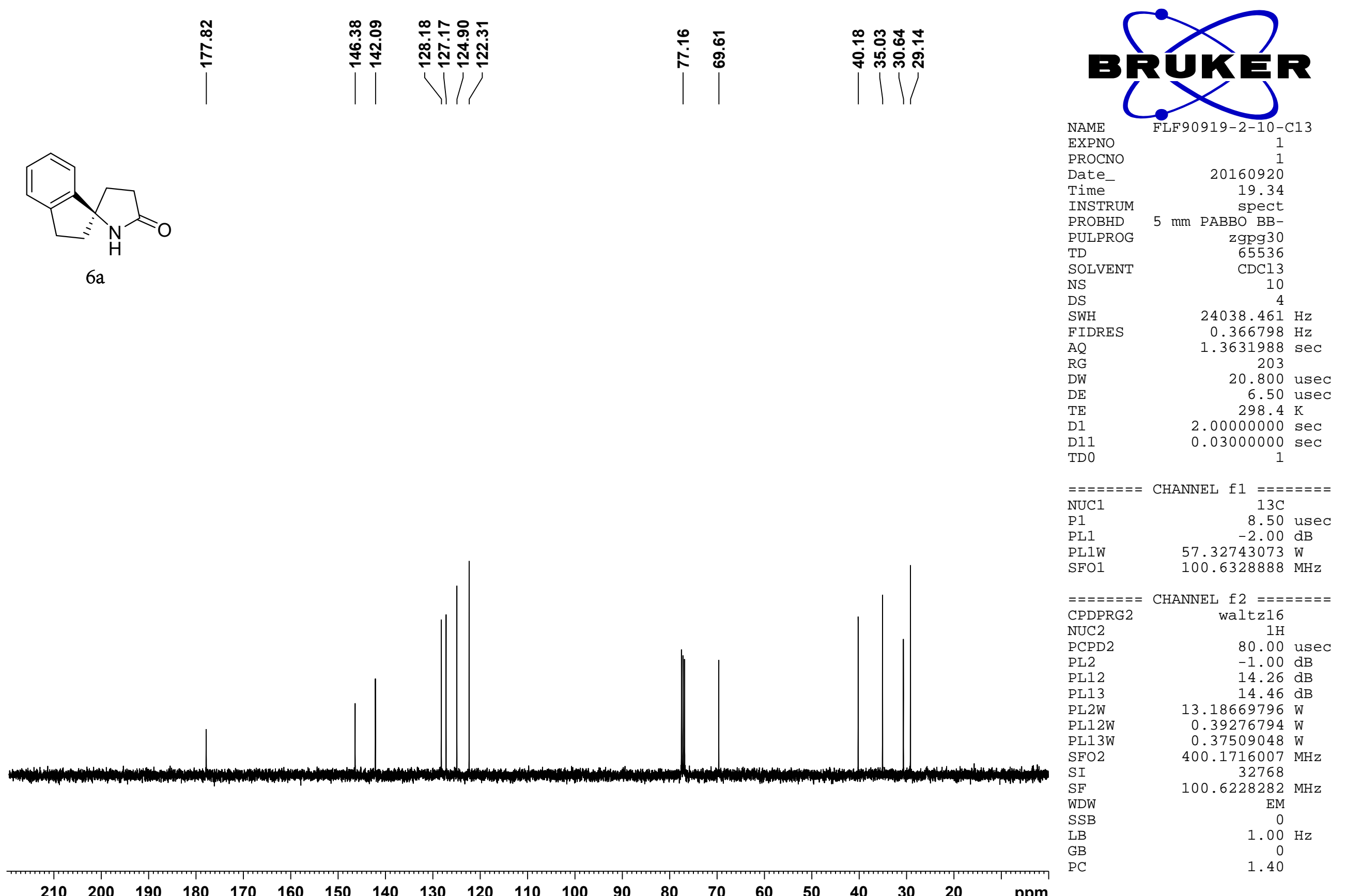



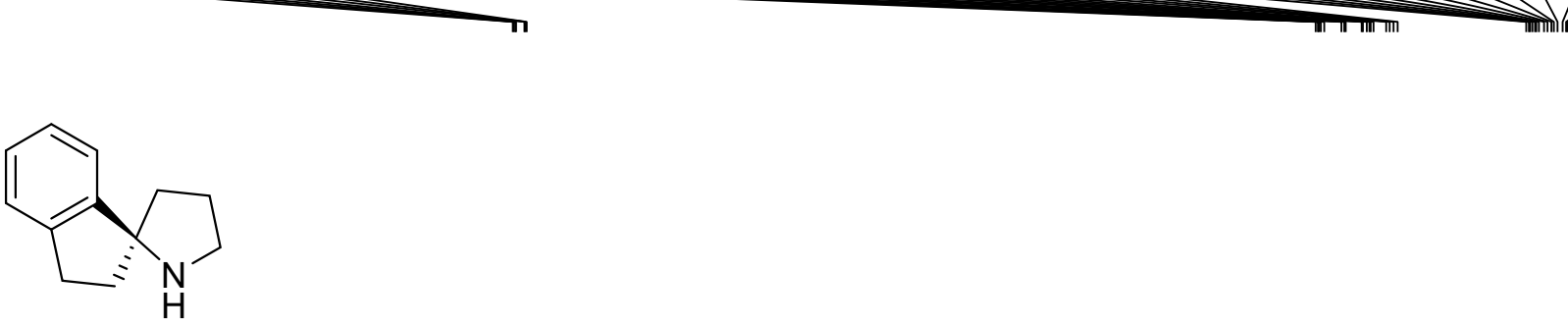

7
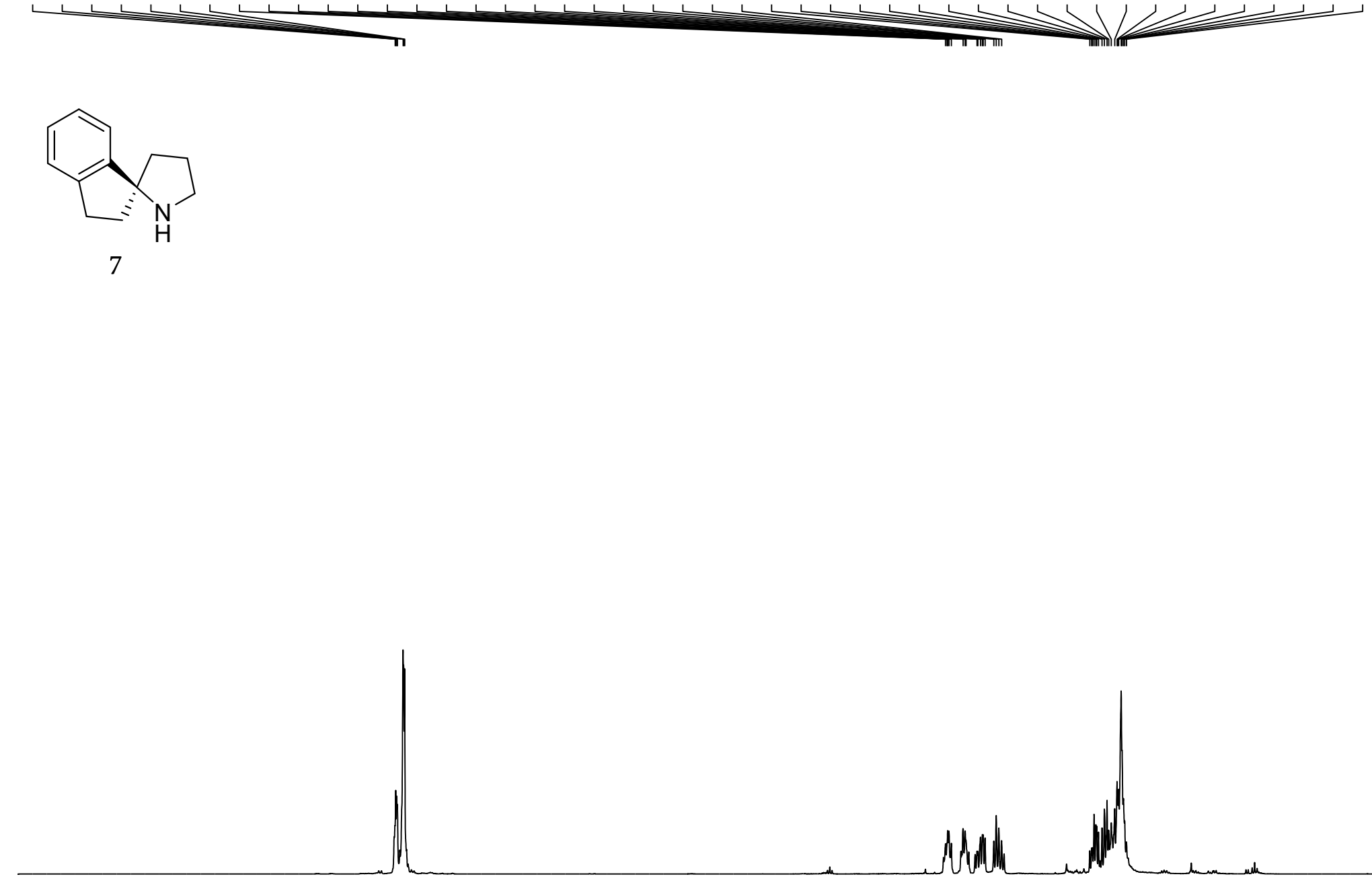

NAME

EXPNO

PROCNO

Date

Time

INSTRUM
PROBHD

PROBHD

PULPRO
TD

TD

NS
DS
SWH

SWH
FIDRES

$\mathrm{AQ}$
$\mathrm{RG}$
$\mathrm{DW}$

DW

TE

D1

Biver

$C_{0}$

FLF161026 - 1-2

20161027 21.45

$5 \mathrm{~mm}$ PABBO BB-

zg30
z 3530

$\mathrm{Zg} 30$
65536

CDC13

16
2

$8223.685 \mathrm{~Hz}$

$0.125483 \mathrm{~Hz}$

$3.9846387 \mathrm{sec}$

25.4

60.800 usec

6.50 usec

$1.00000000 \mathrm{sec}$

$===$
NUC1

P1

PL1

PLIW

SF01

SF

WDW

SSB

B

GB

1

CHANNEL

$======$

13.80 usec

$-1.00 \mathrm{~dB}$

$13.18669796 \mathrm{~W}$

400.1724712 MHz

32768

400.1700181 MHZ

EM
$\Theta$
$0.30 \mathrm{~Hz}$

0.30
1.09

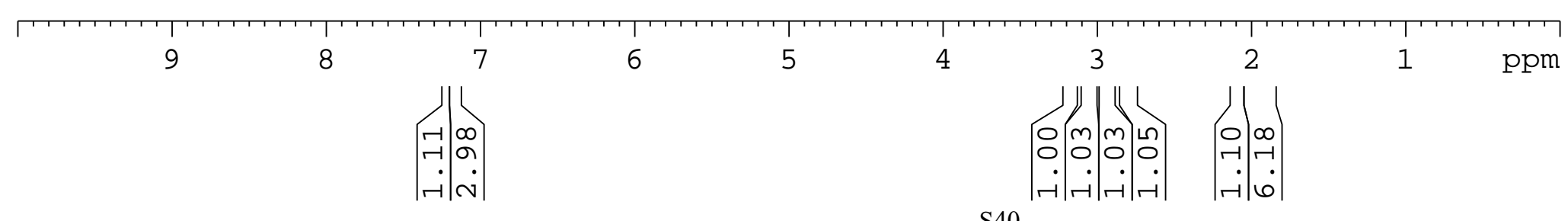




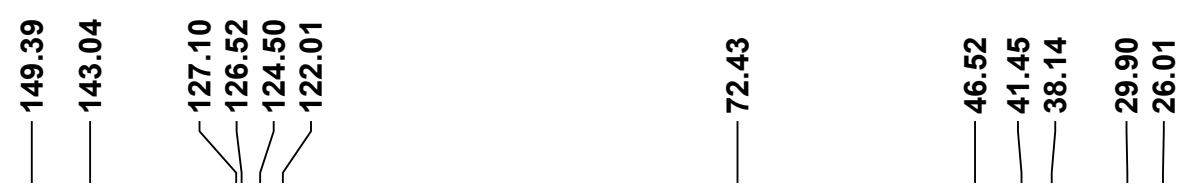

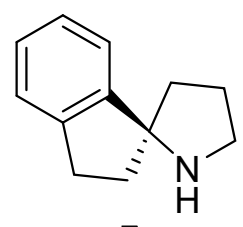

7

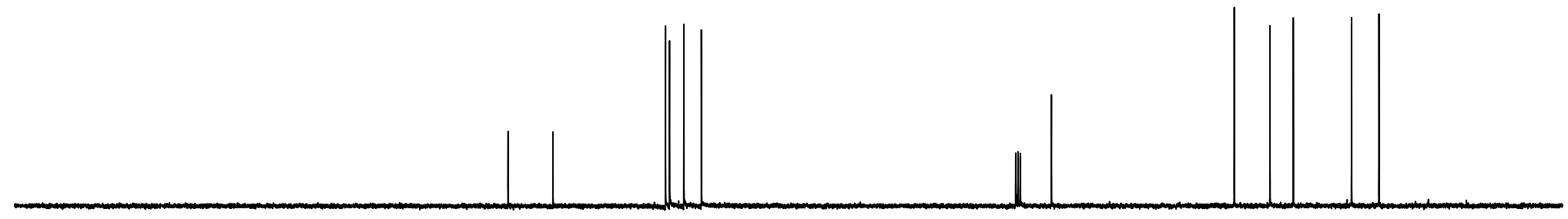

$\begin{array}{lllllllllllllllllllll}210 & 200 & 190 & 180 & 170 & 160 & 150 & 140 & 130 & 120 & 110 & 100 & 90 & 80 & 70 & 60 & 50 & 40 & 30 & 20 & \text { ppm }\end{array}$ 


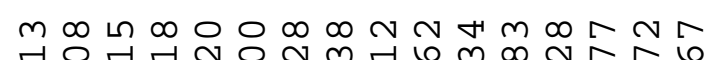

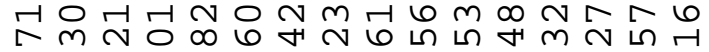
เ ल ल N N ن (<smiles>CCOC(=O)CCC(=O)/C=C/c1cccc(OC)c1</smiles>

Nำ ㅅํ ติ 대패 $\dot{\nabla} \dot{\nabla} \dot{\nabla} \dot{\varnothing} \dot{m} \dot{m} \dot{\sim} \dot{\sim} \dot{N}$ $1 /$

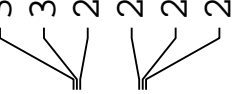

$\infty$ o

N N

में

$1 /$

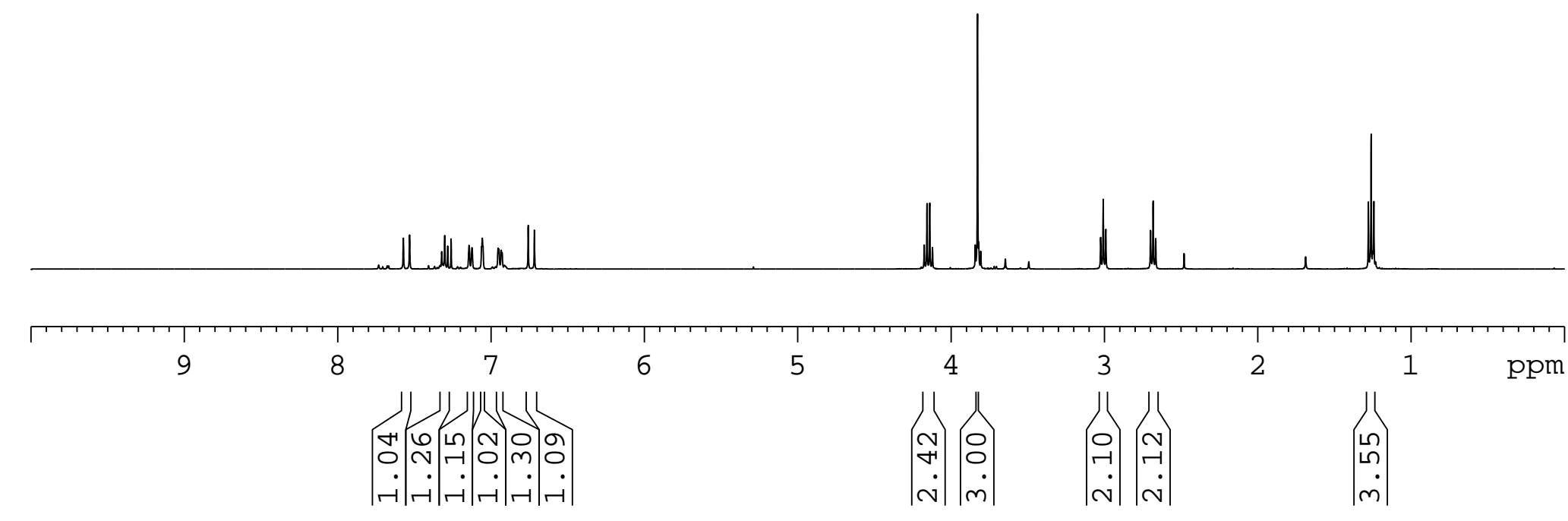

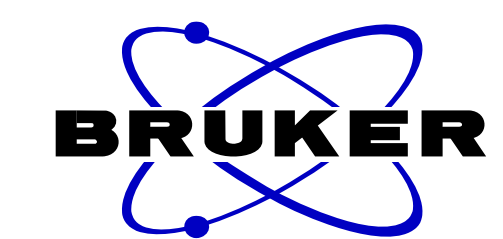

NAME

EXPNO

PROCNO

Date

Time

INSTRUM

PROBHD

PULPROG

TD

SOLVENT

NS
DS
SWH

SWH

$\mathrm{AQ}$
$\mathrm{RG}$

RG

DW

TE

D1

FLF160521

17.42

$5 \mathrm{~mm}$ PABBO BB-

zg30

65536

$\mathrm{CDCl} 13$

16

$8223.685 \mathrm{~Hz}$

$0.125483 \mathrm{~Hz}$

$3.9846387 \mathrm{sec}$ 114

60.800 usec

6.50 use $295.1 \mathrm{~K}$

$1.00000000 \mathrm{sec}$

$=======$ CHANNEL

$\mathrm{NUC1}$

PI

PLIW

SF01

SI

SF

WDW

LB

GB
1

$1 \mathrm{H}$
$13.80 \mathrm{usec}$
$-1.00 \mathrm{~dB}$
$13.18669796 \mathrm{~W}$
$400.1724712 \mathrm{MHz}$
$32768 \mathrm{M}$
$400.1700033 \mathrm{MHz}$
$\mathrm{EM}$
0
$0.30 \mathrm{~Hz}$
0
1.00



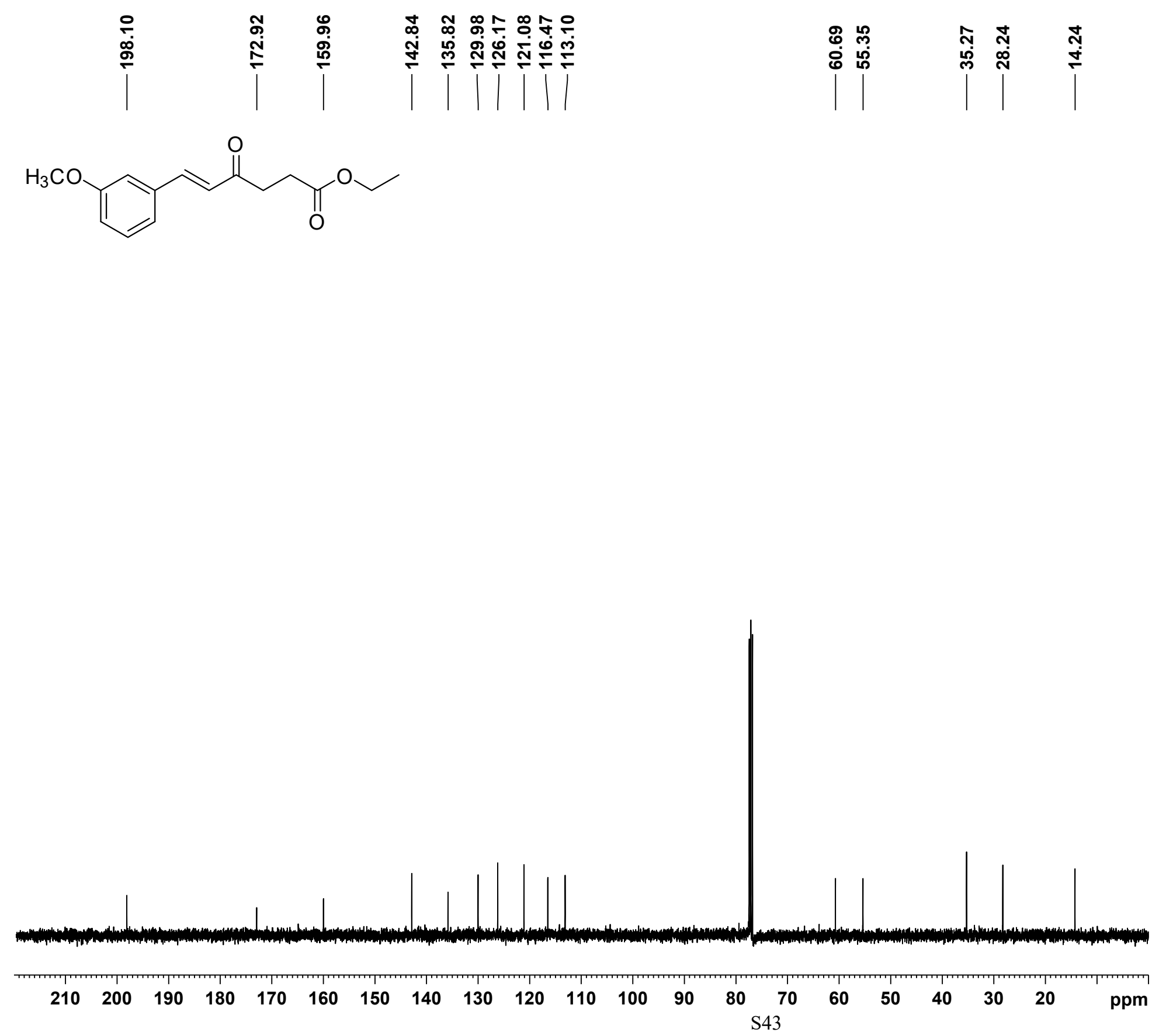
๑ 당

O

……ㄱ.

iriría

3

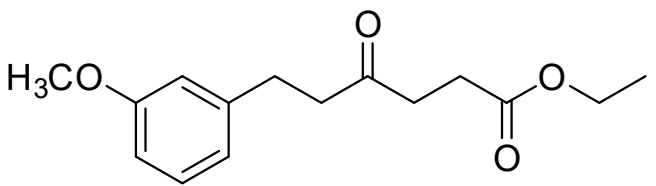

거요

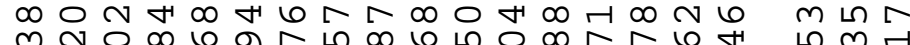

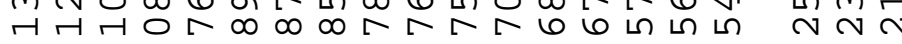
$\dot{\forall} \dot{\forall} \dot{\nabla} \dot{\sim} \dot{N} \dot{N} \dot{N} \dot{N} \dot{N} \sim \vec{N}$
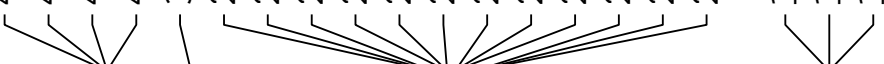

NAME

PROCNO

Date

Time

INSTRUM

PROBHD

PULPROG

TD

SOLVENT

NS

SWH

FIDRES

$\mathrm{AQ}$
$\mathrm{RG}$

RG

DE

TE

D1

$====$ SF01

NUC1

SI

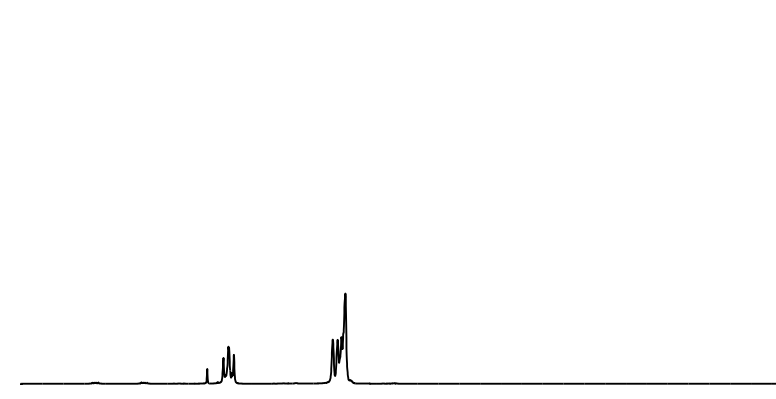

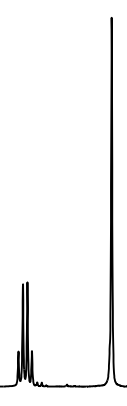
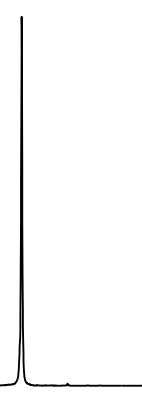

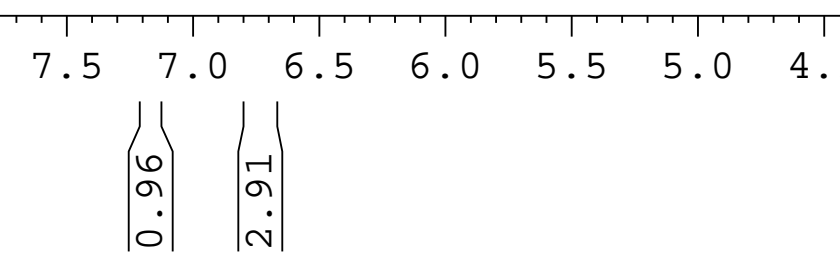

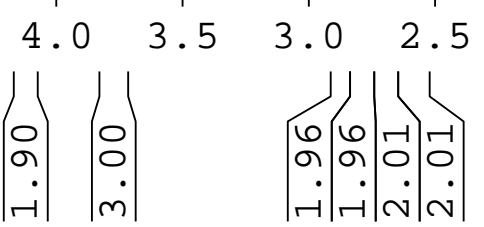

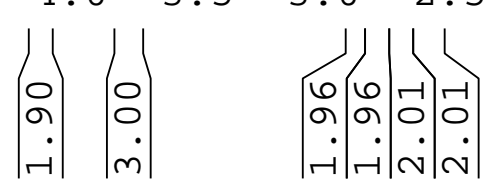

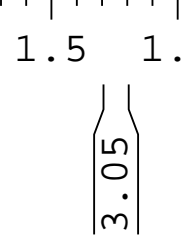

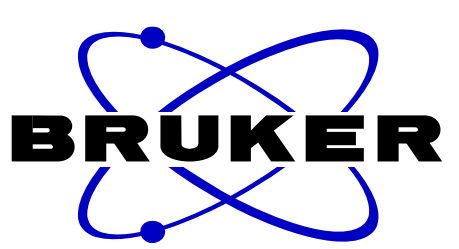

FLF160310-2-pure 1 8.47 $5 \mathrm{~mm}$ PABBO BB$\mathrm{zg} 30$ 65536 $\mathrm{CDCl3}$ 16
2

$8223.685 \mathrm{~Hz}$ $0.125483 \mathrm{~Hz}$ $3.9846387 \mathrm{sec}$ 45.2 60.800 usec $6.50 \mathrm{usec}$

1. $00000000 \mathrm{sec}$

$400.1724712 \mathrm{MHz}$
4. $1 \mathrm{H}$ 13.80 usec 400.1700025 $5 \mathrm{MH}$ EM $\odot .30 \mathrm{~Hz}$ $1.0 \odot$ 

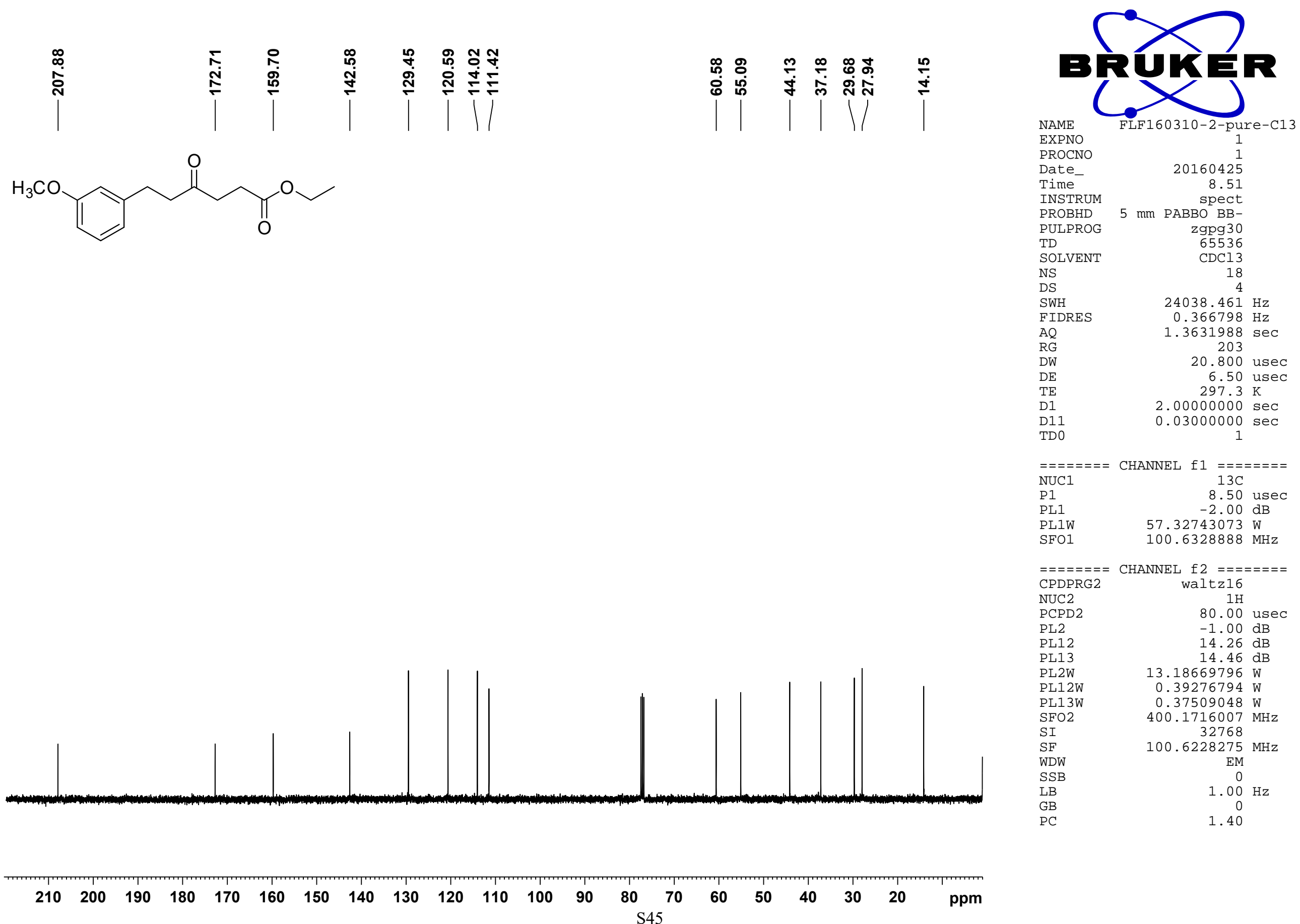
๓

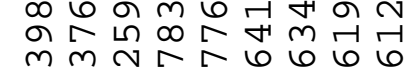
rítio
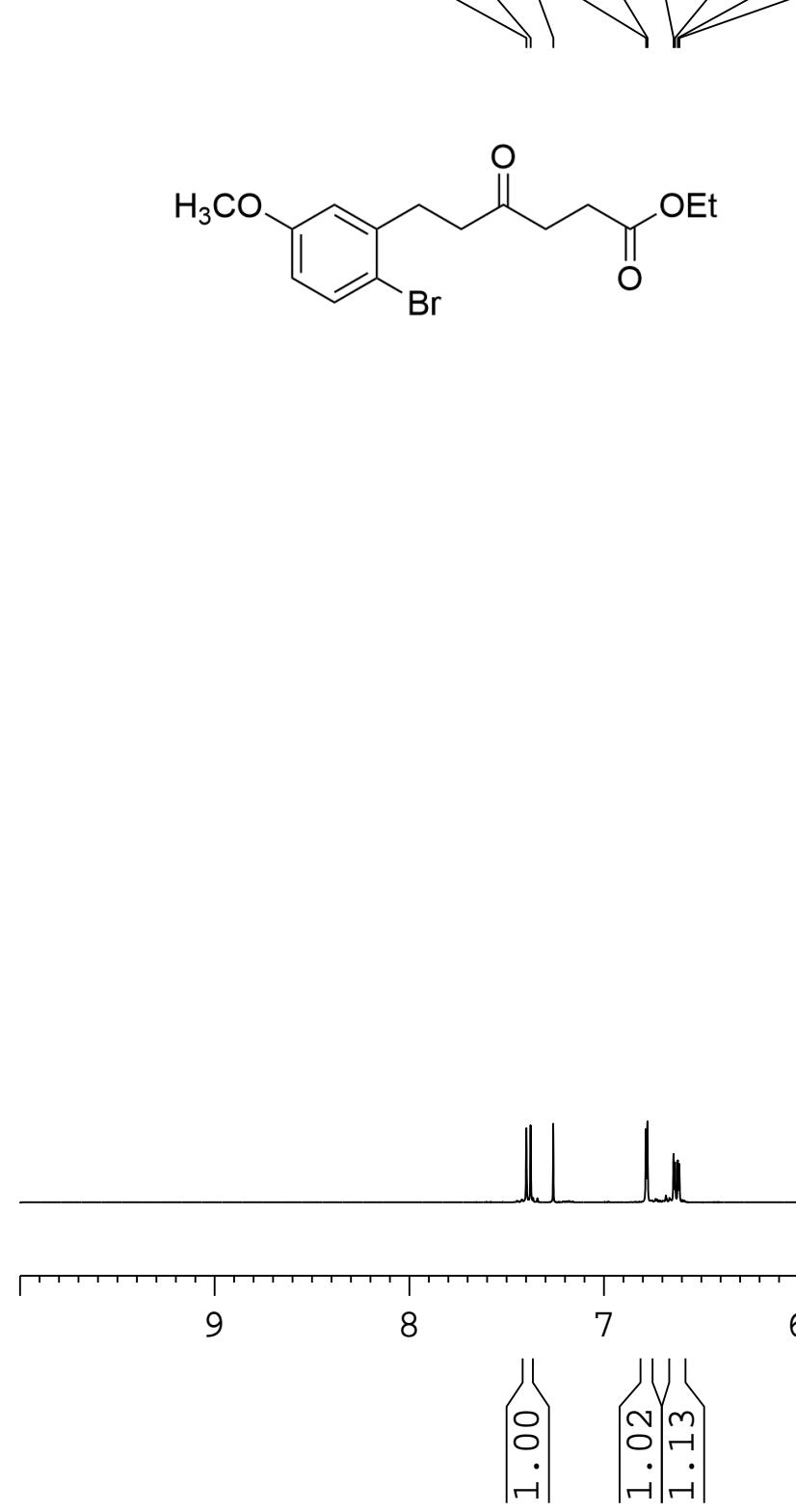

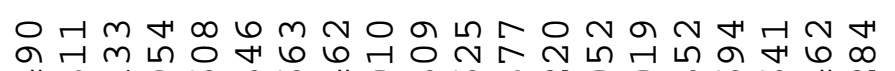
म

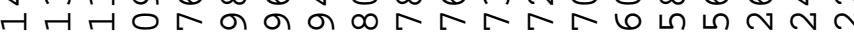

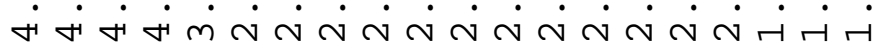
$\longrightarrow$

NAME

EXPNO

PROCNO

Dime

INSTRUM

PROBHD

PULPROG

TD

SOLVENT

NS

SWH

FIDRES

AQ

RG

DE

TE

D1

$====$

NUC1

P1

PL1W

SF01

SI

WDW

SSB

LB

GB

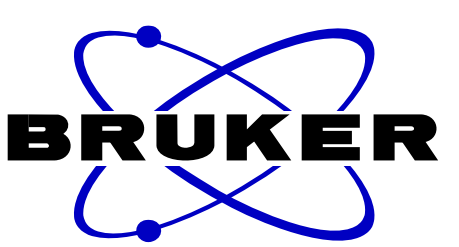

CWG160119-1-PURE

20160121

8.10

$5 \mathrm{~mm}$ PABBO BB-

$\mathrm{zg} 30$

65536

$\mathrm{CDCl}$

16
2

$8223.685 \mathrm{~Hz}$

$0.125483 \mathrm{~Hz}$

$3.9846387 \mathrm{sec}$ 101

60.800 usec

6.50 use

$1.00000000 \mathrm{sec}$

$=$ CHANNEL $f$

1

$======$

13.80 usec

$1.00 \mathrm{~dB}$

$13.18669796 \mathrm{~W}$

$400.1724712 \mathrm{MHz}$

32768

400.1700033 MHZ

EM

$\odot .30 \mathrm{~Hz}$

1. 0 


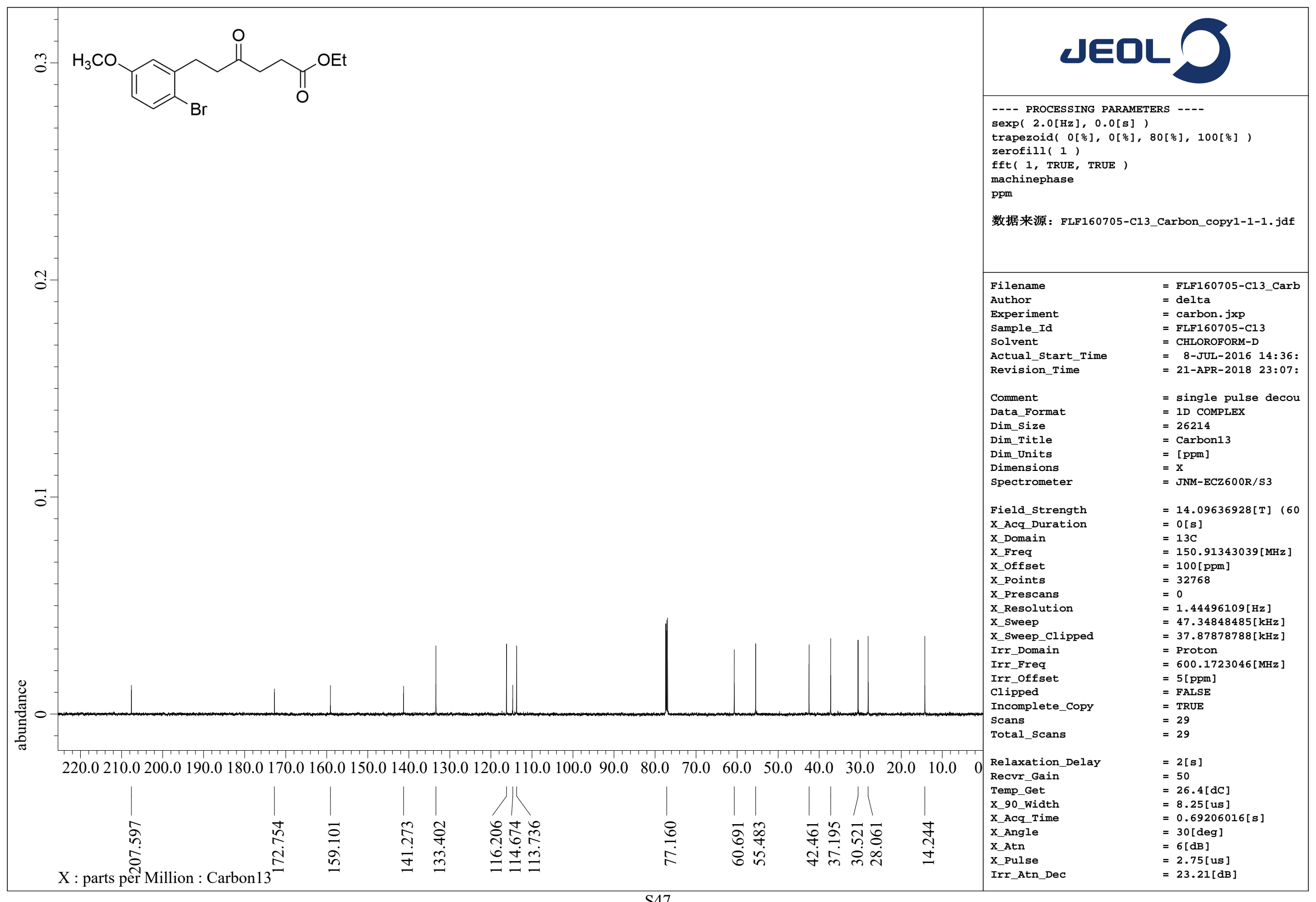




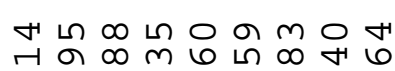

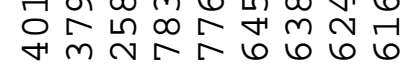

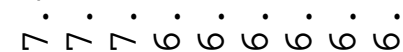

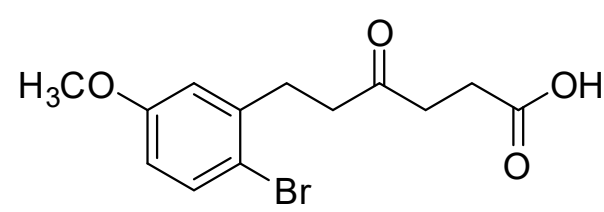

$2 \mathrm{~b}$
นำㅇำ $\infty 000000$

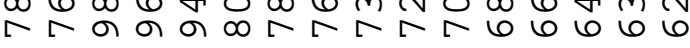

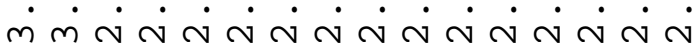

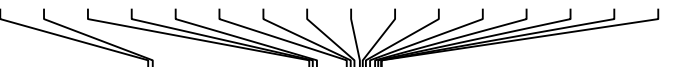

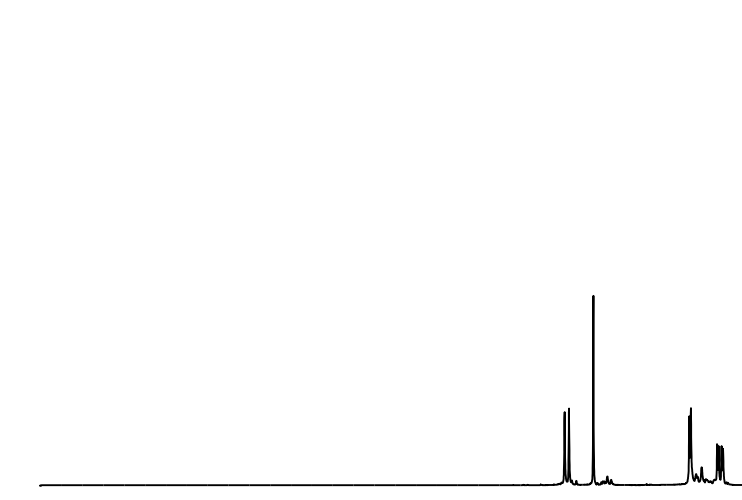

9
7

年
6

5

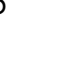

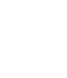

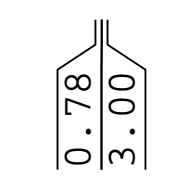

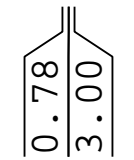

Wh

2

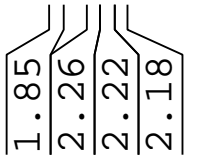

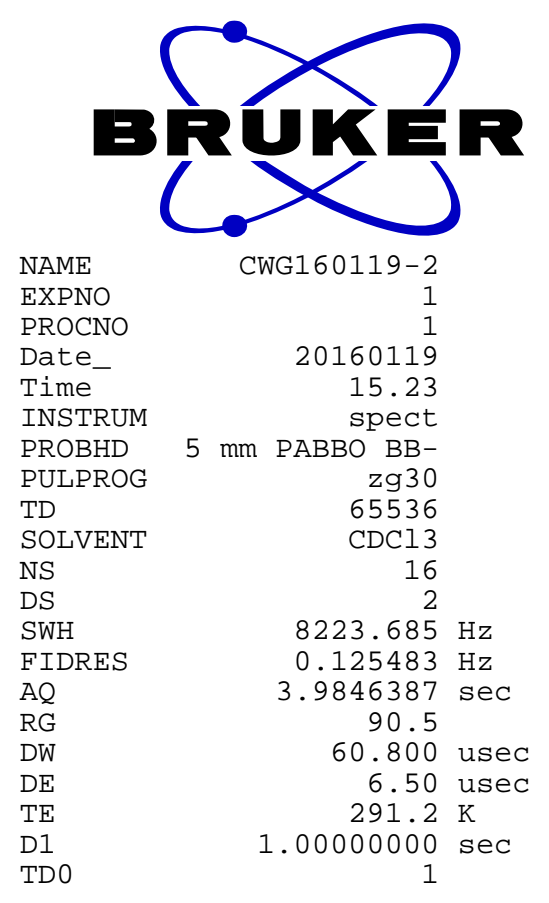

$=======$ CHANNEL NUC1

$=======$

13. 80 usec $-1.00 \mathrm{~dB}$ $13.18669796 \mathrm{~W}$ $400.1724712 \mathrm{MHz}$ 32768 $400.1700037 \mathrm{MHz}$ EM $0.30 \mathrm{~Hz}$ 1. 00 


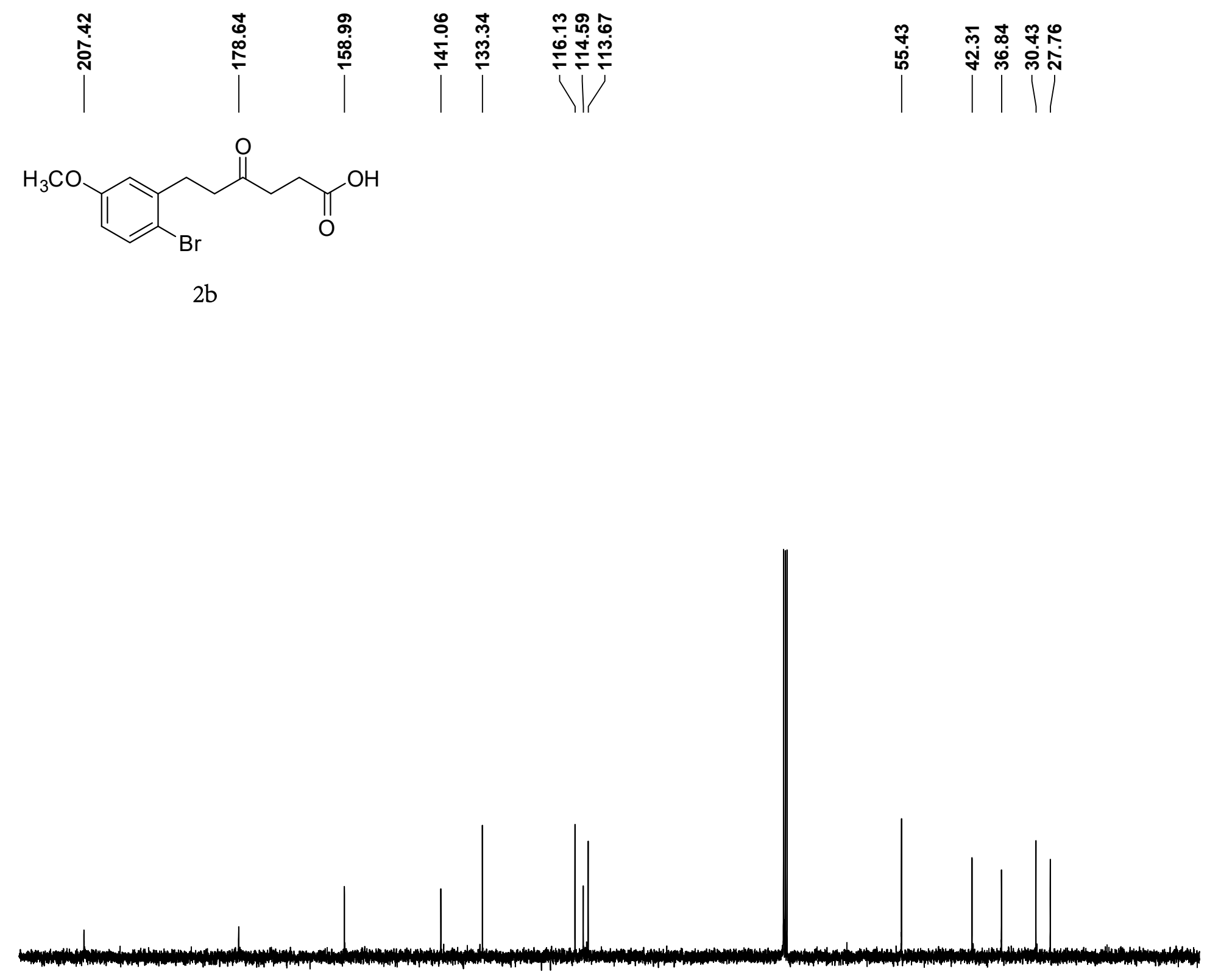 BR
NAME FLF160414-2-C13 EXPNO PROCNO Date
Time
INSTRUM
PROBHD
PULPROG
TD
NS
DS
SWH
FIDRES
$A Q$
RG
DW
DE
TE
D1
D11
TD
20160414
20.59
$5 \mathrm{~mm}$ РАВВO BВ-
zgpg30
$-9 p g 30$
65536
CDC13
4
$24038.461 \mathrm{~Hz}$
$0.366798 \mathrm{~Hz}$
$1.3631988 \mathrm{sec}$ 203
20.800 usec
6.50 usec $296.4 \mathrm{~K}$
2. $00000000 \mathrm{se}$ $0.03000000 \mathrm{sec}$ 1

$===$ NUC1

PL1W

\section{SF01}

CHANNEL $f 1$

(1)

CPDPRG2
CPCI

NUC2

PCPD2

PL2
PL12

PL13

PL2W

PL12W

PL13W

SI

SF

WDW

LB

LB

CHANNEL $f$

$====$

.50 usec

$57.32743073 \mathrm{~d}$

$100.6328888 \mathrm{MHz}$

f2 ========

waltz16

80.0० usec

$-1.00 \mathrm{~dB}$

$14.26 \mathrm{~dB}$

$14.26 \mathrm{~dB}$
$14.46 \mathrm{~dB}$

$13.18669796 \mathrm{~W}$

13.18669796
0.39276794

$0.37509048 \mathrm{~W}$

$400.1716007 \mathrm{MHz}$

42768

$100.6228275 \mathrm{MHz}$

EM

$\odot$
$1 . \odot$
$\odot$
$\ominus$

1.40 
の N

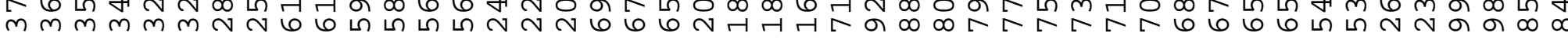

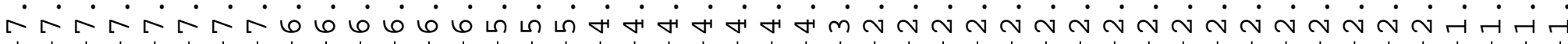

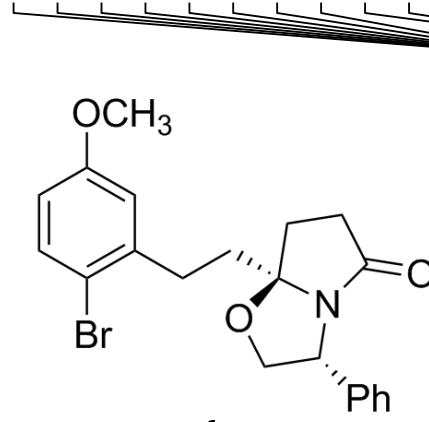

$3 \mathrm{~b}$

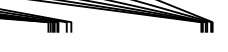

NAME

EXPNO

PROCNO

Date

Time

INSTRUM

PROBHD

PULPROG

TD

SOLVENT

NS
DS
SWH

SWH
FIDRES

$\mathrm{AQ}$
$\mathrm{RG}$
$\mathrm{DW}$

DW

TE

D1

Bitiker

$\underbrace{}_{F L F 160312-X-3}$

20160315 11.08

$5 \mathrm{~mm}$ PABBO BB-

zg30

65536

$\mathrm{CDCl} 3$

16
2

$8223.685 \mathrm{~Hz}$

$0.125483 \mathrm{~Hz}$

$3.9846387 \mathrm{sec}$ 203

60.800 usec

6.50 use

$1.0 \odot \odot \odot \odot \odot \odot \odot \mathrm{sec}$

$====$

P1

PL1

PL1W

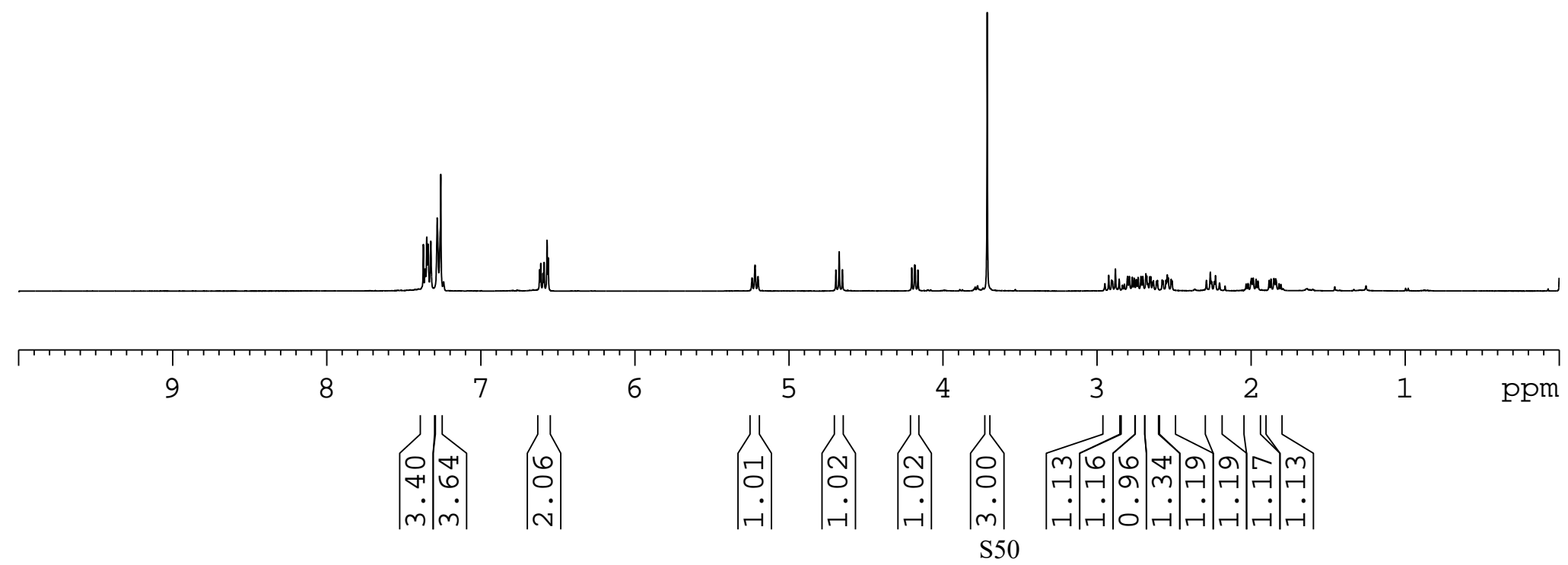

13.80 usec

$-1.00 \mathrm{~dB}$
$13.18669796 \mathrm{~W}$

$400.1724712 \mathrm{MHz}$

32768

400.1700036 MHz

EM

$\odot .30 \mathrm{~Hz}$

1.00 


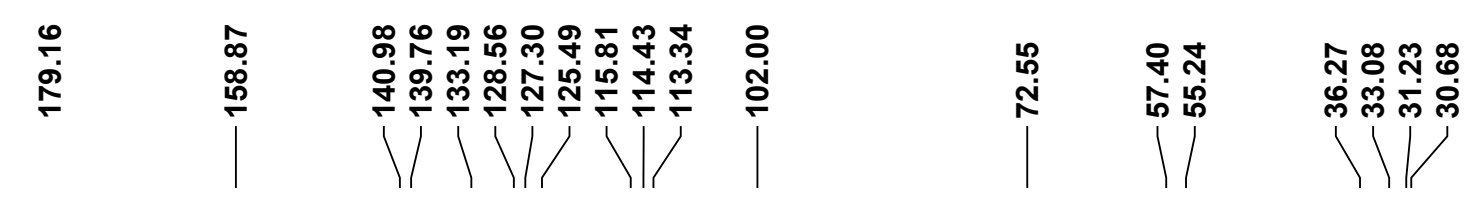

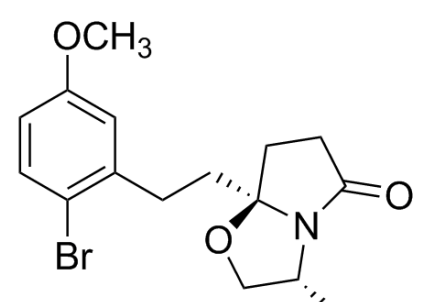

"Ph

$3 b$

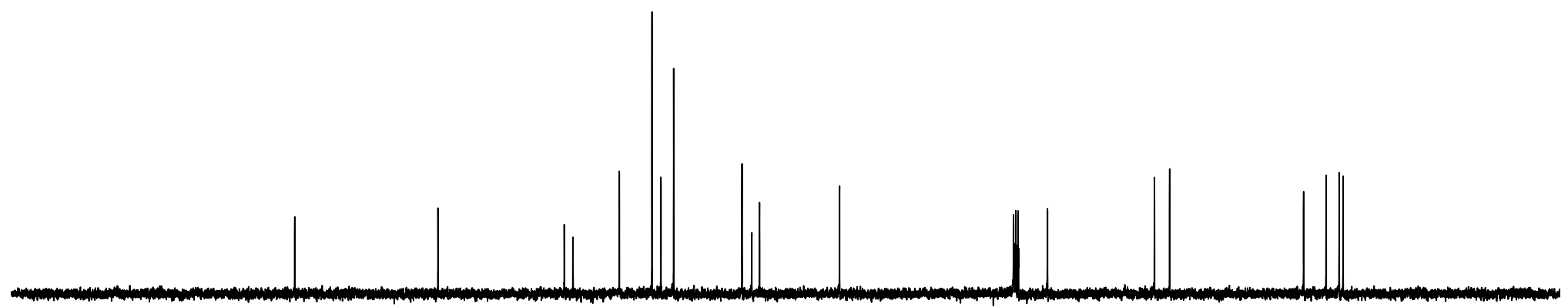

CPDPRG2
NUC2
PCPD2
PL2
PL12
PL13
PL2W
PL12W
PL13W
SF02
SI
SF
WDW
SSB
LB
GB
PC

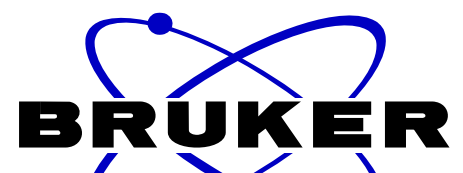
NAME FLF160414-3-1P-C13
EXPNO
PROCNO
Date
INSTRUM
PROBHD
PULPROG
TD
NS
DS
SWH
FIDRES
$A Q$
RG
DW
TE
D1
TDO
2016042
21.54
$\mathrm{mm}$ PABBOct
spect
$B O$ BB-

zgpg30
65536
CDC13
14
4
$24038.461 \mathrm{~Hz}$
$0.366798 \mathrm{~Hz}$
$1.3631988 \mathrm{sec}$
203
.800 usec
6.50 usec
$296.9 \mathrm{~K}$
$2.00000000 \mathrm{sec}$
$0.03000000 \mathrm{sec}$

NUC1

P1

PL1

SF01

1 =======

$13 \mathrm{C}$

.

$57.32743073 \mathrm{~W}$

$100.6328888 \mathrm{MHz}$

$==$ CHANNEL f2 =======

PDPRG

SF

GC

waltz16
wall

8๑. $1 \mathrm{H}$ usec

80.00 usec

$-1.00 \mathrm{~dB}$

$14.26 \mathrm{~dB}$

$13.18669796 \mathrm{~W}$

13. $186276794 \mathrm{~W}$

$0.37509048 \mathrm{~W}$

$400.1716007 \mathrm{MHz}$

32768

$100.6228437 \mathrm{MHz}$

EM

$\Theta$
$1.0 \ominus \mathrm{Hz}$
$\Theta$
$1.4 \Theta$ 


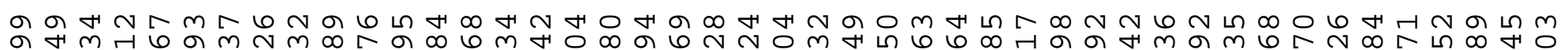
出

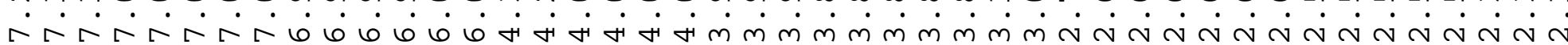

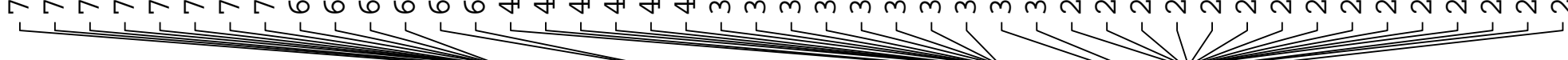<smiles>COc1ccc(Br)c2c1[C@@]1(CCC(=O)N1[C@H](CO)c1ccccc1)CC2</smiles>
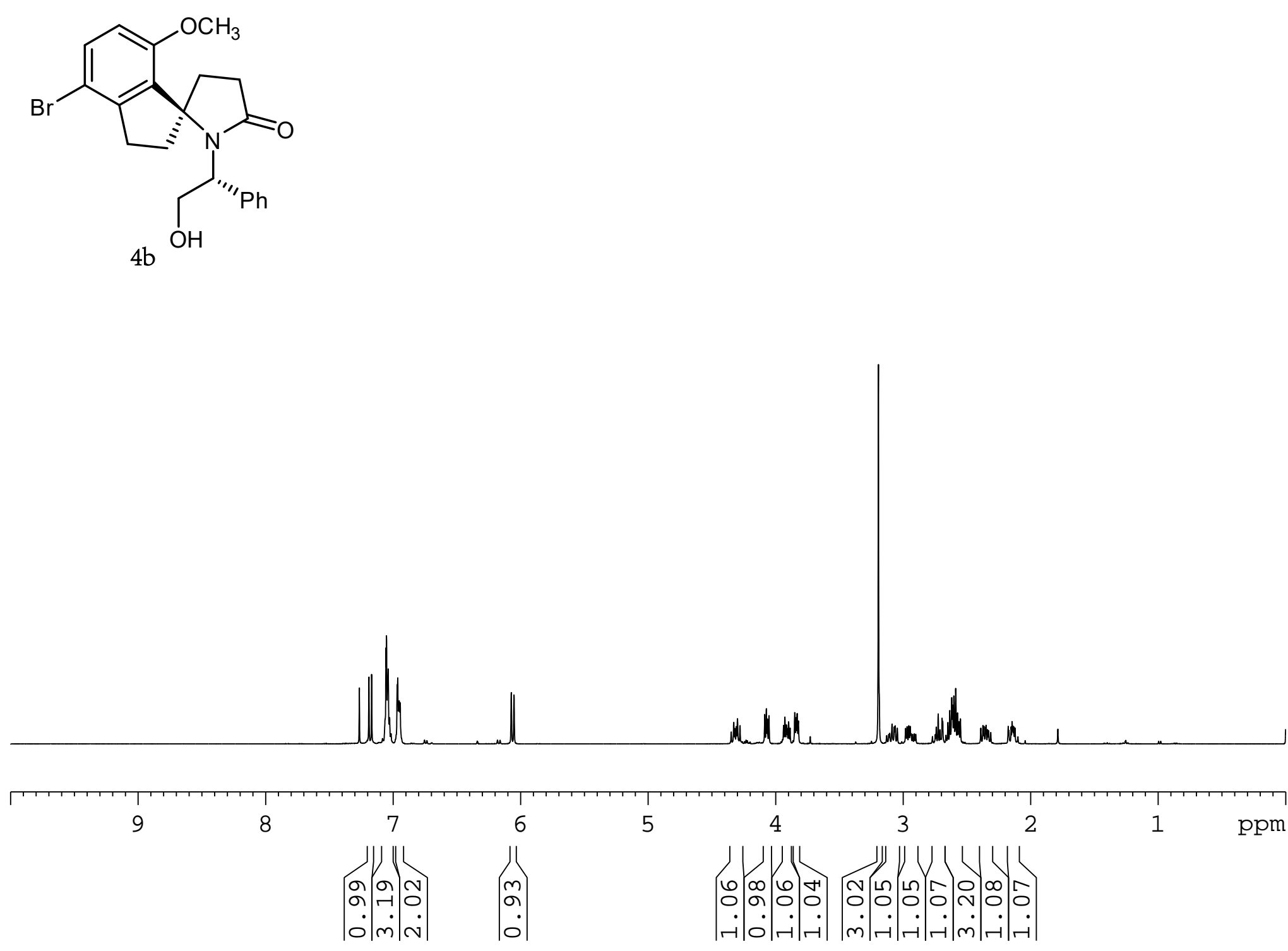

NAME

EXPNO

PROCNO

Time

INSTRUM

PROBHD

PULPROG

TD

SOLVENT

DS

SWH

FIDRES

AQ

$\mathrm{DW}$

DE

D1

TDO

NUC1

P1

PL1

SF01

SI

SF

WSW

LB

PC

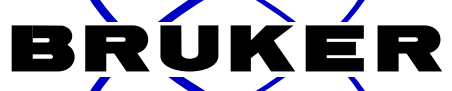

$\underbrace{}_{\text {FLF160312-X-1-PURE }}$ 1

20160316 21.53 $5 \mathrm{~mm}$ PABBO BBzg30 65536 $\mathrm{CDCl} 3$ 16
2

$8223.685 \mathrm{~Hz}$ $0.125483 \mathrm{HZ}$ $3.9846387 \mathrm{sec}$ 114

60.800 usec 6.50 use (95.5 K

$1.00000000 \mathrm{sec}$

1

$\begin{aligned} & 13.8 \odot \text { usec } \\ &-1.0 \odot \mathrm{dB} \\ & 13.18669796 \mathrm{~W} \\ & 400.1724712 \mathrm{MHz} \\ & 32768 \mathrm{MHz} \\ & 400.1700011 \mathrm{MHz} \\ & \mathrm{EM} \\ & \odot \\ & \odot .3 \odot \mathrm{Hz} \\ & \odot \\ & 1.0 \odot\end{aligned}$



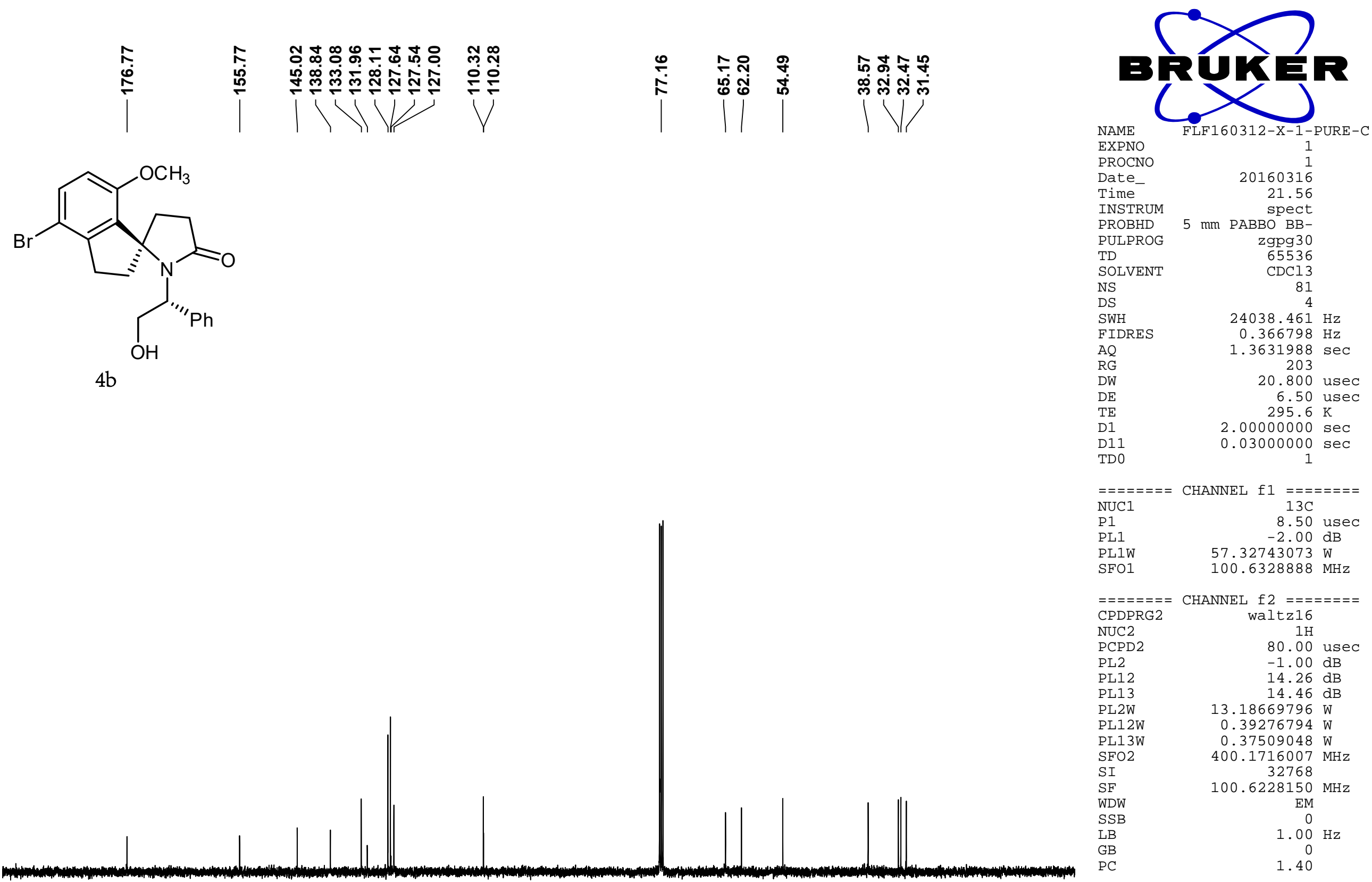

$190 \quad 180$ 


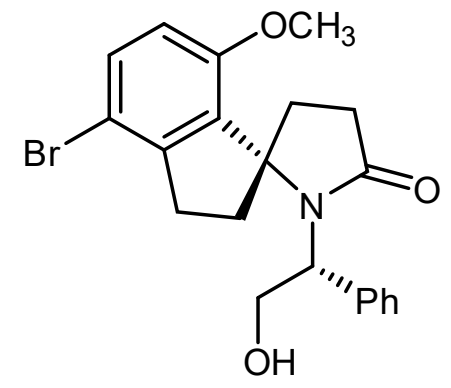

$4 \mathrm{~b}$
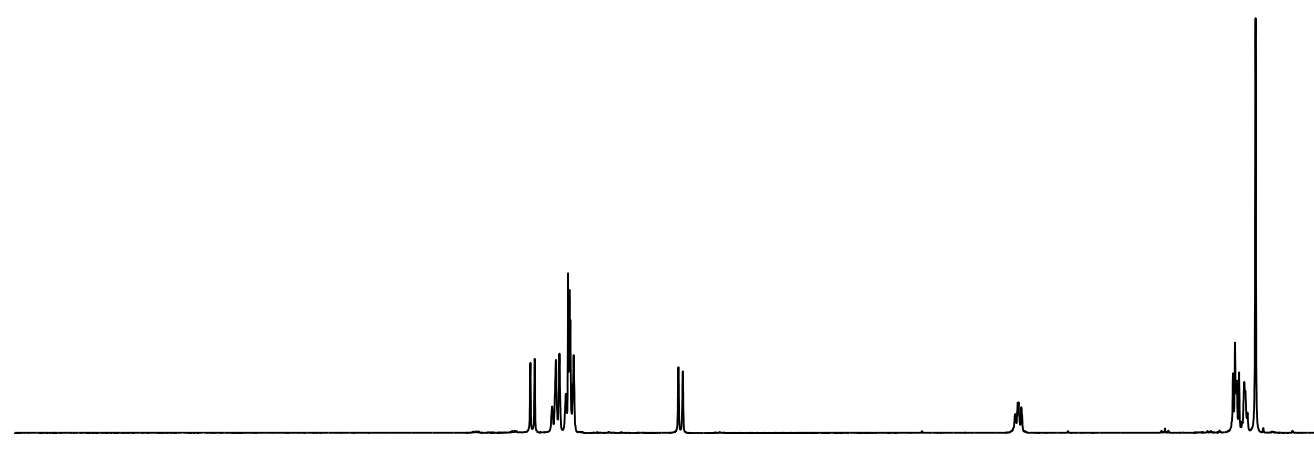

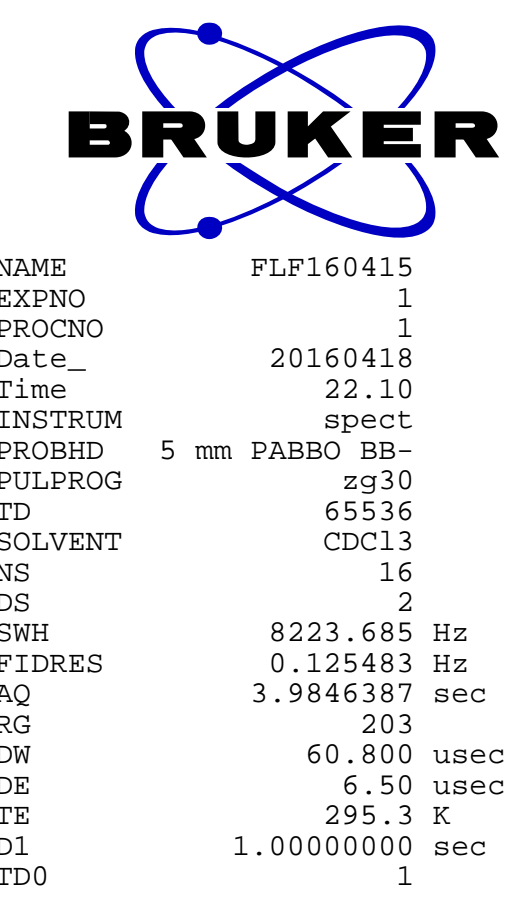

$===$

NUC1

P1

PL1W

SF01

SFO
SF

SF

WDW

LB

GB

PC
13.80 usec $13.18669796 \mathrm{~W}$ 32768 $400.1700033 \mathrm{MHz}$ EM $1.0 \odot$ $400.1724712 \mathrm{MHz}$ $\odot .30 \mathrm{~Hz}$ Mon aud 
<smiles>COc1ccc(Br)c2c1[C@]1(CCC(=O)N1[C@H](CO)c1ccccc1)CC2</smiles>

$4 b^{\prime}$

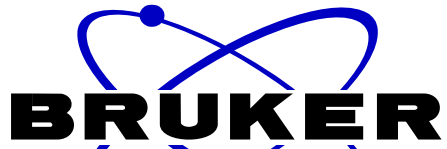 $\underbrace{}_{\text {FLF160312-X-1-PURE-C }}$} EXPNO EXPNO

Date

Time-

INSTRUM

PROBHD

PLPRO

TD

SOLVENT

NS

SWH

FIDRES

AQ

RG

$\mathrm{DW}$
$\mathrm{DE}$
$\mathrm{TE}$

D1

D11

20160316 21.56

$5 \mathrm{~mm}$ spect

zgpg30

$\operatorname{CDCl} 3$

81

\section{$24038.461 \mathrm{~Hz}$}

$24038.461 \mathrm{~Hz}$

$1.3631988 \mathrm{~Hz}$

203

20.800 usec

6.50 usec

$2.00000000 \mathrm{sec}$

$0.03000000 \mathrm{sec}$

$=======$ CHANNEL $\mathrm{f} 1$

P1

PL1

SF01

$$
13 \mathrm{C}
$$

$13 \mathrm{C}$ usec

$-2.00 \mathrm{~dB}$
$57.32743073 \mathrm{~W}$

$100.6328888 \mathrm{MHz}$

$========$ CHANNEL f2 ========

CPDPRG2

PCPD2

PCPD2

PL12

PL13

PL2W

PL12W

PL13W

SF02

SI

SF

SSB

LB
GB
PC

waltz16
$1 \mathrm{H}$
$80.00 \mathrm{usec}$
$-1.00 \mathrm{~dB}$
$14.26 \mathrm{~dB}$
$14.46 \mathrm{~dB}$
$13.18669796 \mathrm{~W}$
$0.39276794 \mathrm{~W}$
$0.37509048 \mathrm{~W}$
$400.1716007 \mathrm{MHz}$
$32768 \mathrm{H}$
$100.6228150 \mathrm{MHz}$
$\mathrm{EM}$
$\odot$
$1.00 \mathrm{~Hz}$
$\odot$
1.40




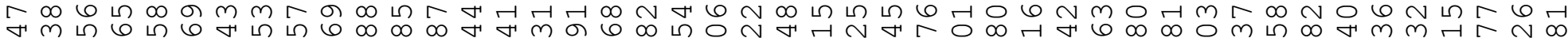

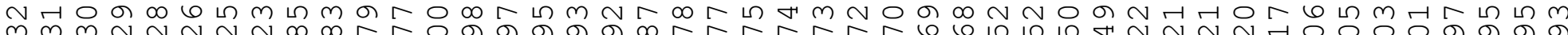

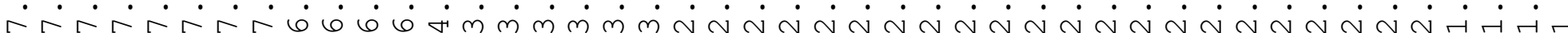
$\longrightarrow$
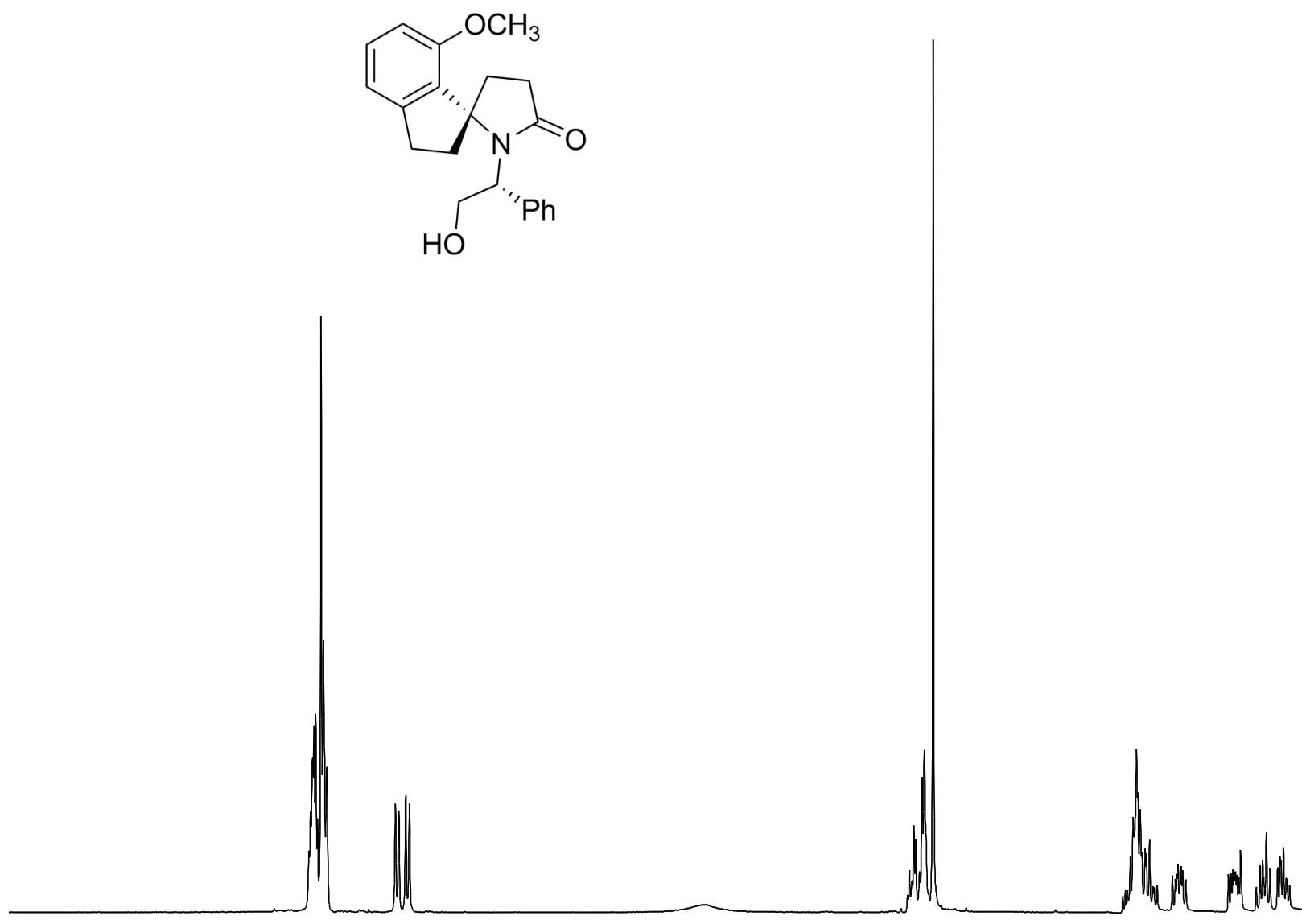

\section{NAME}

PROCNO

Date

Time-

INSTRUM

PROBHD

PULPROG

SOLVENT

NS

DS

SWH
FIDRES

AQ

$\mathrm{RG}$

DW

$\mathrm{DE}$

TE

D1 0

$=======$ CHANNEL $\mathrm{f} 1$
NUC1

P1
PL1
PL1W

SFO

SI

WDW

SSB

GB

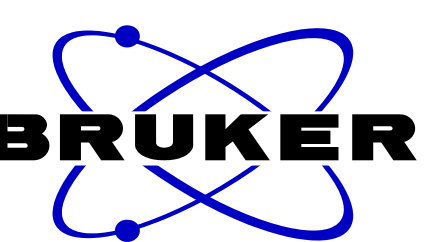

LHJ171204-H

20180328

10.22

$\mathrm{mm}$ PABBO $\mathrm{BB}$

zg30

65536

$\mathrm{CDCl} 3$

$16163.793 \mathrm{~Hz}$

$0.246640 \mathrm{~Hz}$

$2.0272970 \mathrm{sec}$

30.933 use

6.50 usec

$300.5 \mathrm{~K}$

$1.00000000 \mathrm{sec}$

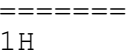

15.50 usec

$-1.00 \mathrm{~dB}$

$13.18669796 \mathrm{~W}$

$400.1728012 \mathrm{MHz}$

$400.1700000 \mathrm{MHz}$

$$
\begin{array}{r}
0 \\
0.30 \mathrm{~Hz} \\
0 \\
1.00
\end{array}
$$$$
\text { EM }
$$

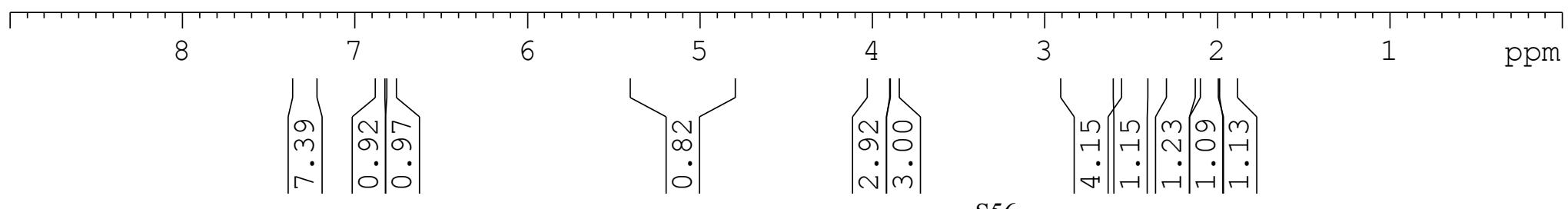



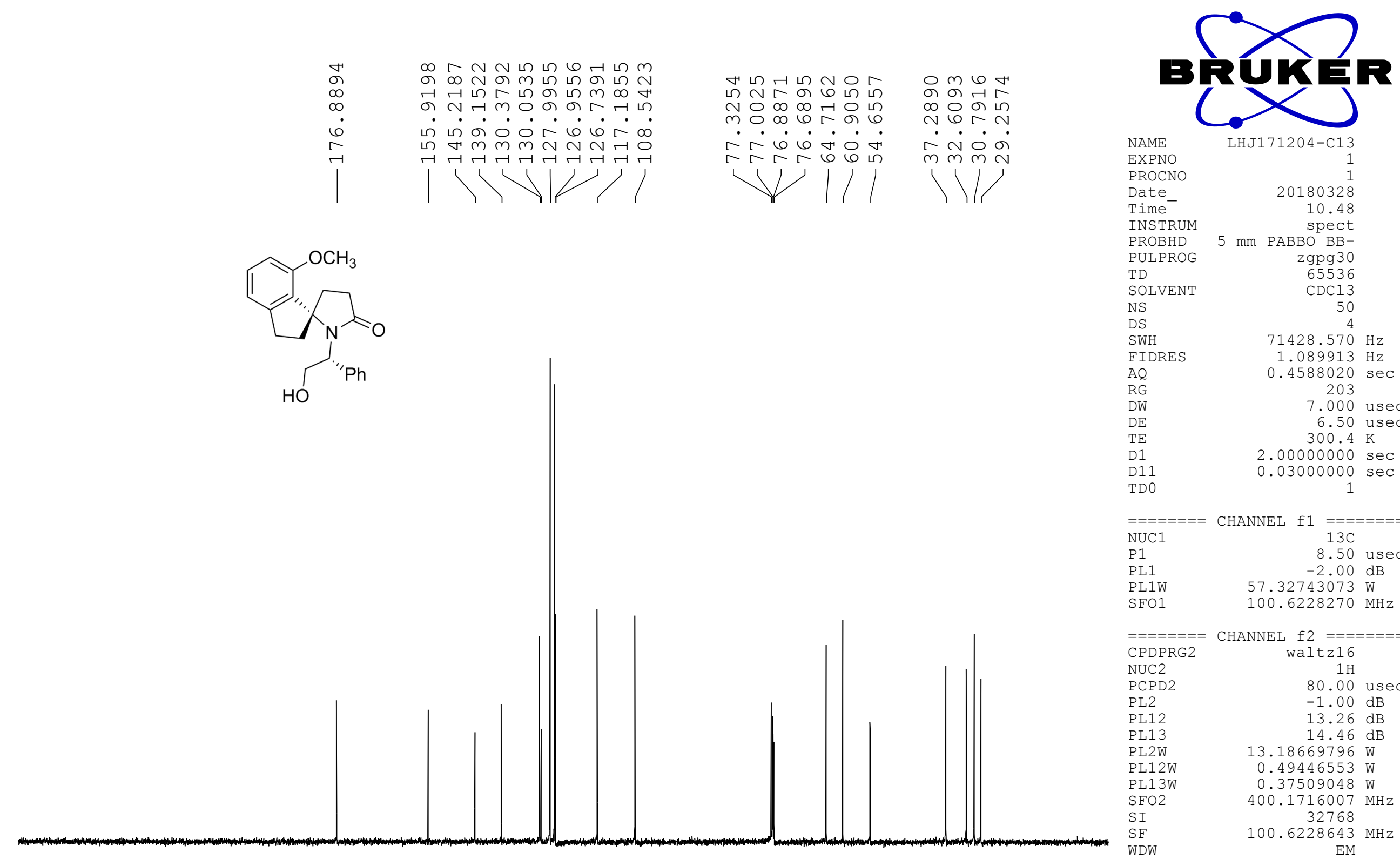

NAME EXPNO

PROCNO

Date

INSTRUM

PROBHD

PULPROG

SOLVENT

NS

DS

SWH
FIDRES

FIDRES

$\mathrm{AQ}$
$\mathrm{RG}$

RG

$\mathrm{DE}$

TE

D1

D1 1
TD 0

LHJ1 $71204-C 13$

20180328 10.48

$========$ CHANNEL f 1 $========$ $\mathrm{NUC1}$

PL1

PL1W

zgpg30
65536
CDC13

4
$71428.570 \mathrm{~Hz}$

$1.089913 \mathrm{~Hz}$

$0.4588020 \mathrm{sec}$

203

7.000 usec

6.50 usec

$300.4 \mathrm{~K}$

$2.00000000 \mathrm{sec}$

$0.03000000 \mathrm{sec}$

CPDPRG

NUC2

PCPD2

PL2

PL12

PL13

PL $12 \mathrm{~W}$

PL13W

SI

SI

WDW
.50 usec
$-2.00 \mathrm{~dB}$
$57.32743073 \mathrm{~W}$

$100.6228270 \mathrm{MHz}$

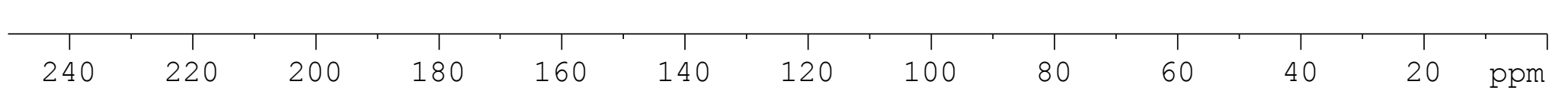

LB
GB

HANNLL $2=======$
waltz16
$1 \mathrm{H}$
$80.00 \mathrm{usec}$
$-1.00 \mathrm{~dB}$
$13.26 \mathrm{~dB}$
$14.46 \mathrm{~dB}$
$13.1866996 \mathrm{~W}$
$0.49446553 \mathrm{~W}$
$0.37509048 \mathrm{~W}$
$400.1716007 \mathrm{MHz}$
32768
$100.6228643 \mathrm{MHz}$
$\mathrm{EM}$
0
$1.00 \mathrm{~Hz}$
0
1.40



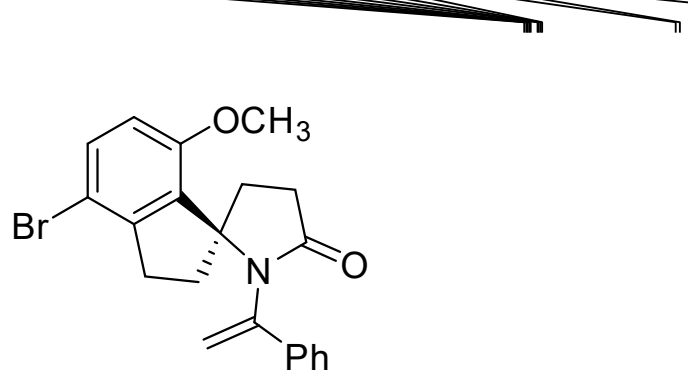

NAME

EXPNO

PROCNO

Date

Time

INSTRUM

PROBHD

PULPROG

$5 b$

SOLVENT

NS

DS

SWH

FIDRES

$\mathrm{AQ}$
$\mathrm{RG}$
$\mathrm{DW}$

DW

TE

D1

apiner

$\underbrace{}_{\text {FLF160822-23-1 }}$

20160824

9.03

$5 \mathrm{~mm}$ РАBBO BB-

$\mathrm{zg} 30$
65536

CDCl3

16

$8223.685 \mathrm{~Hz}$

$0.125483 \mathrm{~Hz}$

$3.9846387 \mathrm{sec}$ 203

60.800 usec $6.50 \mathrm{usec}$

$1.00000000 \mathrm{sec}$

$=======$ CHANNEL NUC1

1

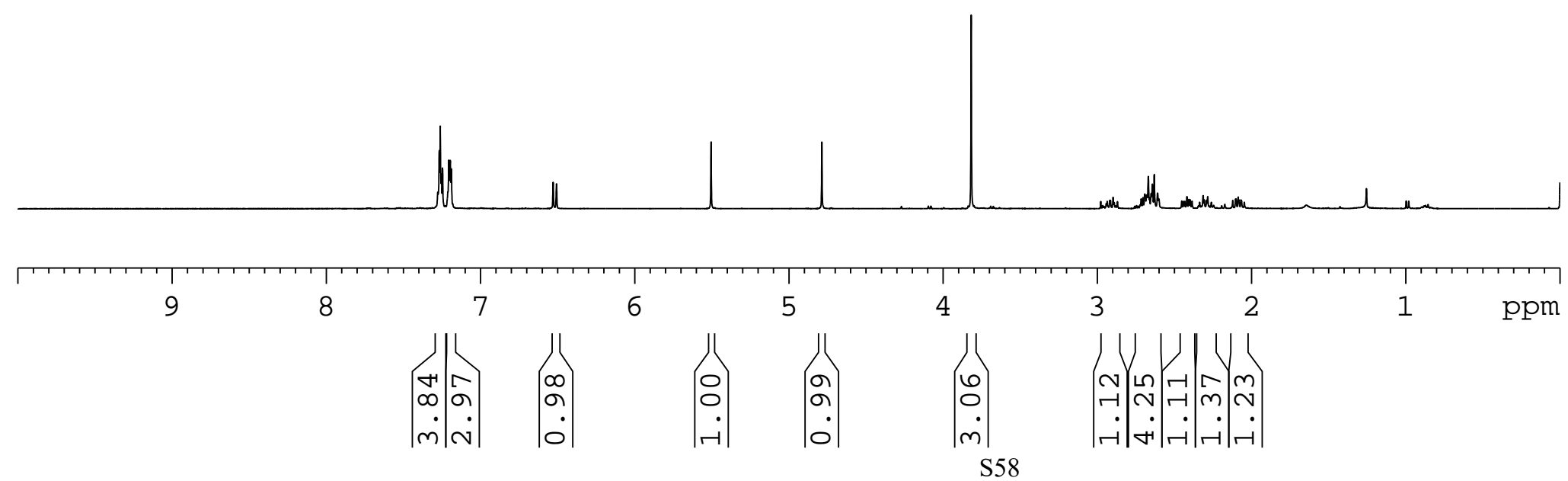

$13.18669796 \mathrm{~W}$

$400.1724712 \mathrm{MHz}$

32768
400.1700028

$\mathrm{EM}$
0
$\odot .30 \mathrm{~Hz}$
0
1.00




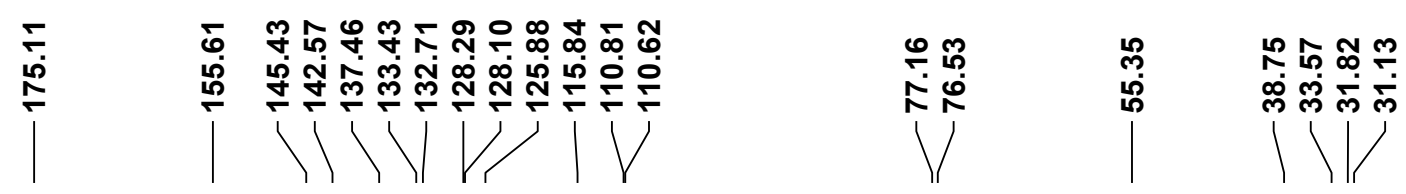

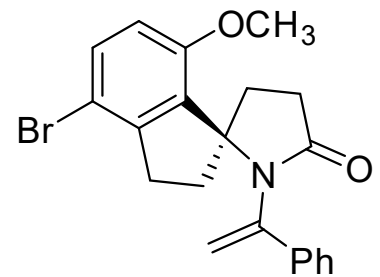

$5 \mathrm{~b}$

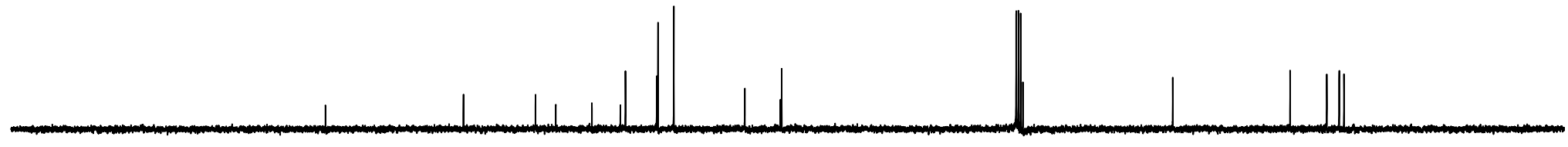

210200
क्रिए NAME EXPNO PROCNO Date
Time
INSTRUM
PROBHD
PULPROG
TD
NS
DS
SWH
FIDRES
$A Q$
RG
DW
TE
D1
TD
LF160822-23-C13
20160823
21.22
$5 \mathrm{~mm}$ РАВBO BB-
zgpg30
$\mathrm{gpg} 30$
65536
$\mathrm{CDCl} 3$
32
$24038.461 \mathrm{~Hz}$
$0.366798 \mathrm{~Hz}$
$1.3631988 \mathrm{sec}$ 203
20.800 usec
6.50 usec
$299.8 \mathrm{~K}$
$2.00000000 \mathrm{sec}$
$0.03000000 \mathrm{sec}$

$========$ CHANNEL f1 ======== NUC1

PL1

PL1W

$13 \mathrm{C}$ usec

$2.00 \mathrm{~dB}$

$========$ CHANNEL f2 =======

CPDPRG2 waltz16

NUC2

NUC2

PL2

PL12

PL13

PL2W
PL12W

PL12W

PL13W

$\mathrm{SI}$

SF

WDW

$S S B$

LB
GB
PC

8๑. $\stackrel{1 \mathrm{H}}{ }$ usec

$80.0 \odot$ usec
$-1.00 \mathrm{~dB}$

$14.26 \mathrm{~dB}$

$13.18669796 \mathrm{~W}$

$13.18669796 \mathrm{~W}$

$\odot .37509048 \mathrm{~W}$

$400.1716007 \mathrm{MHz}$

32768

$100.6228187 \mathrm{MHz}$

EM

$\odot$
$1 . \odot$
$\odot$
$\ominus$

1.40 
걱

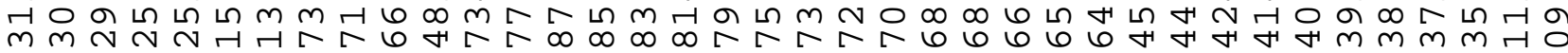
NR
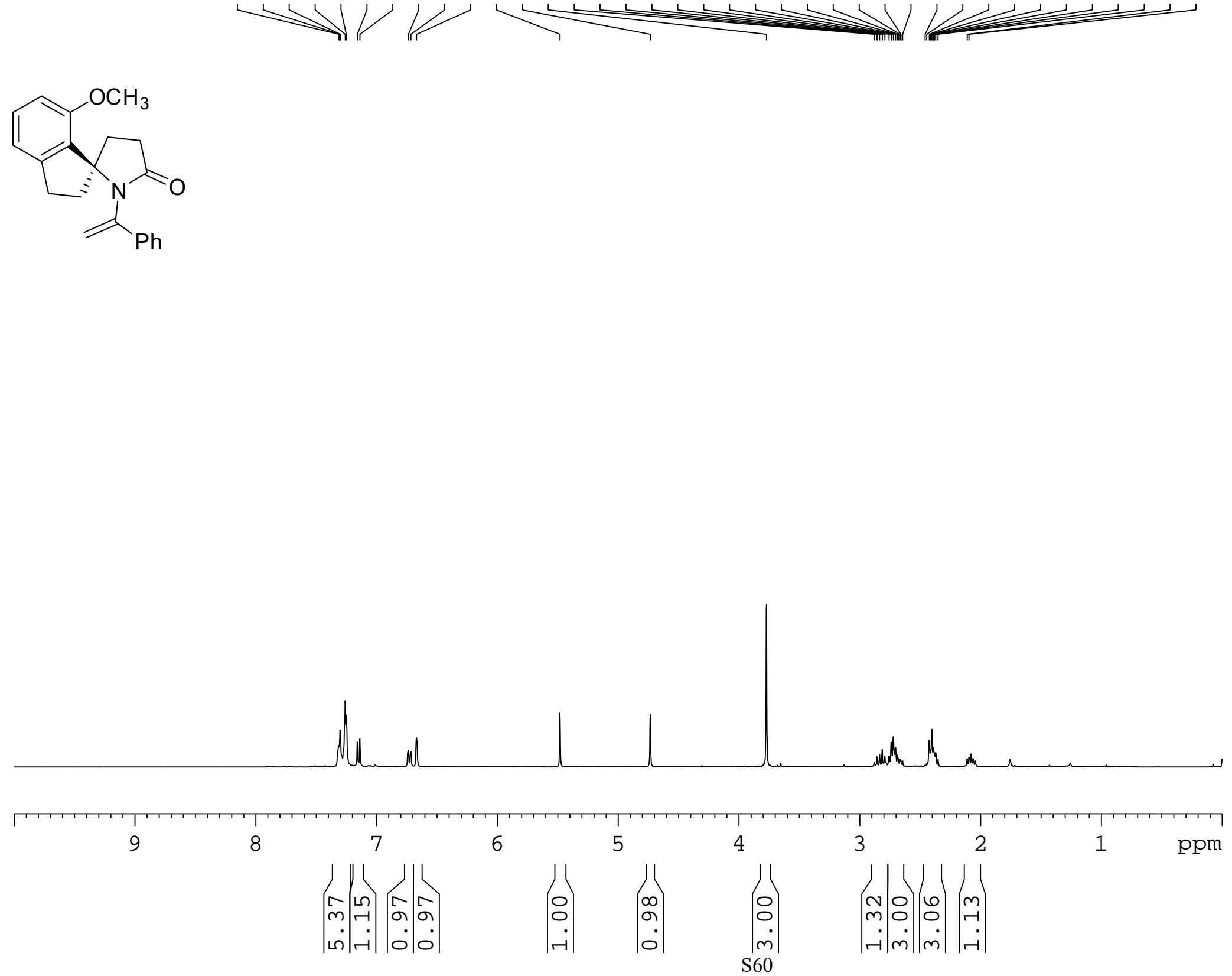

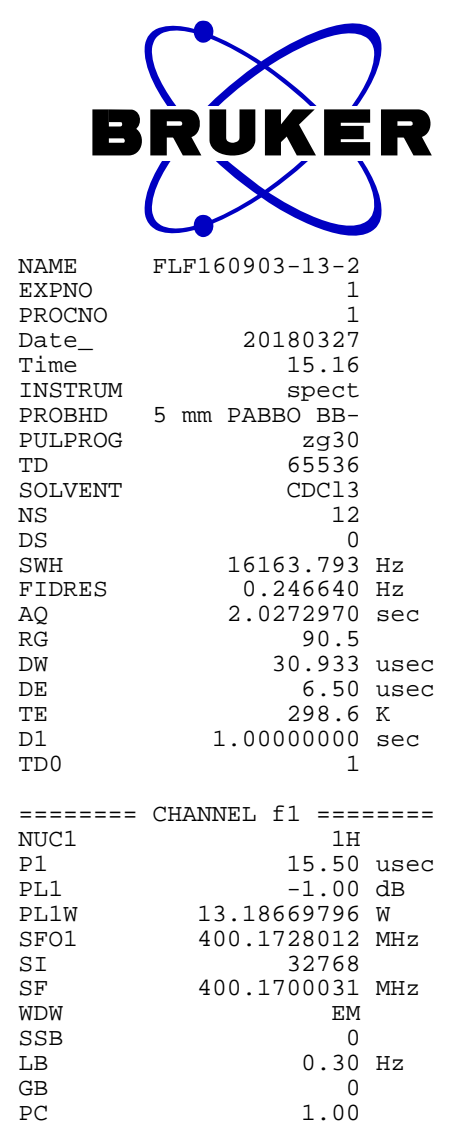




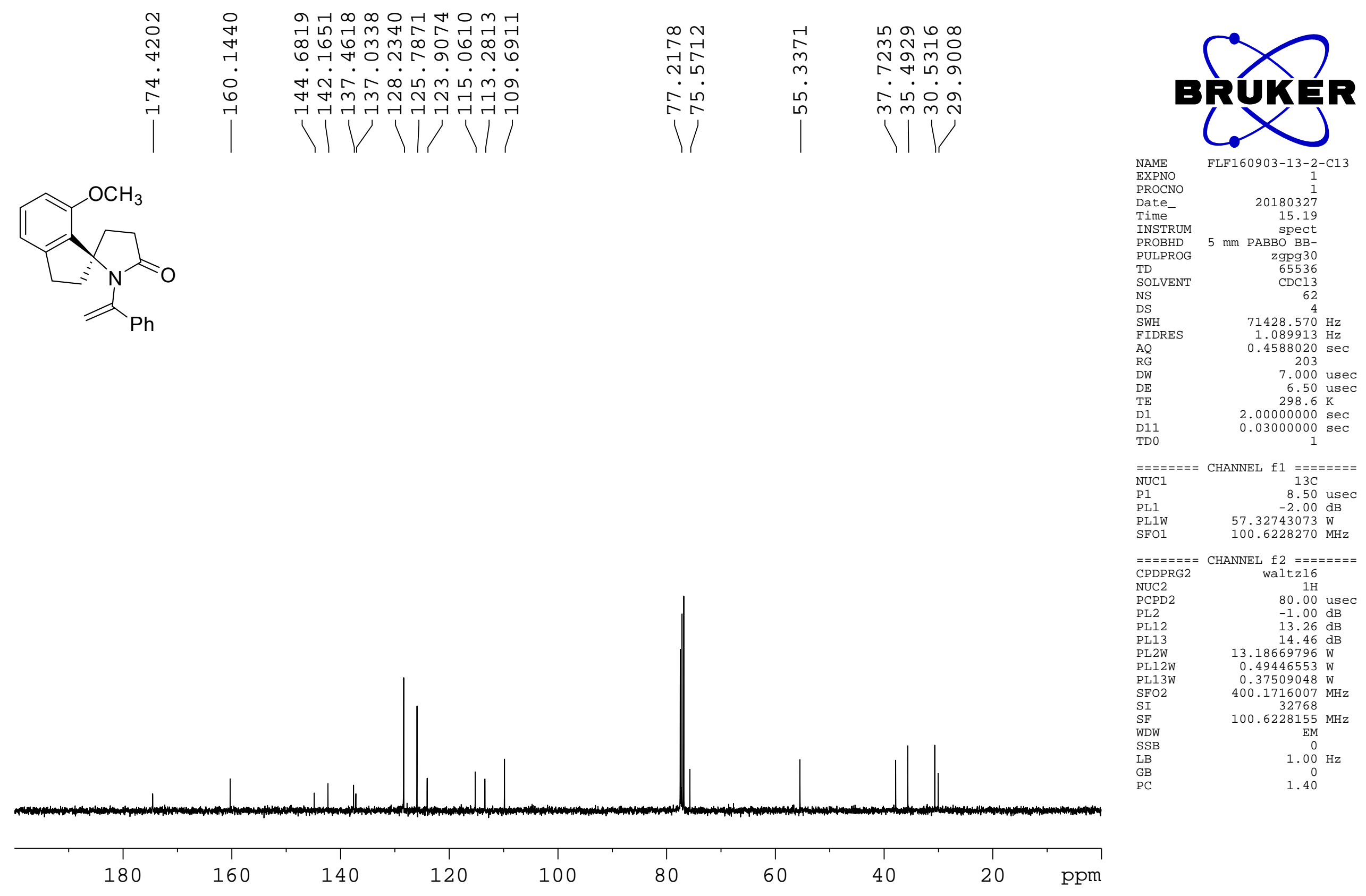




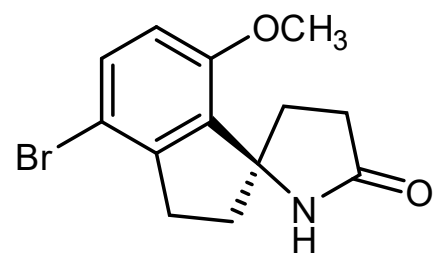

$6 \mathrm{~b}$
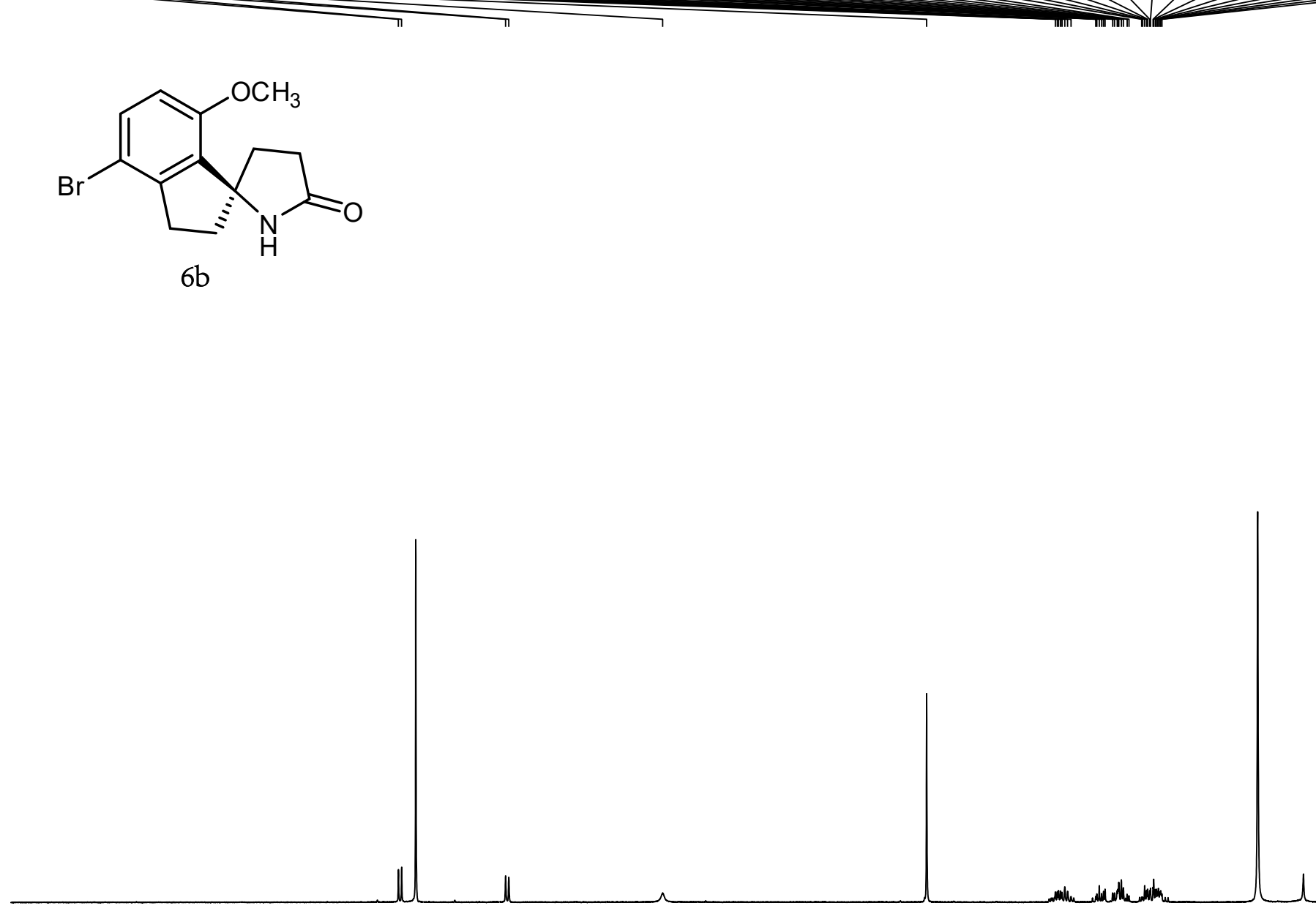
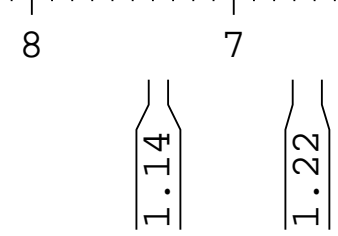

6

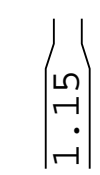

5

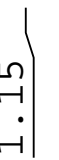

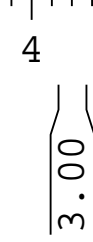

2

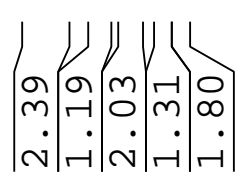

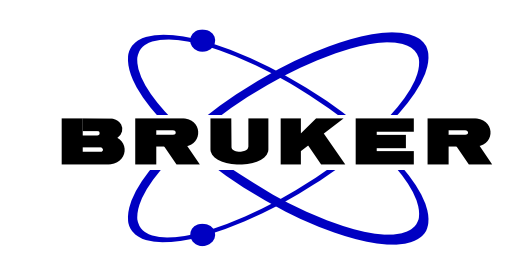

NAME

EXPNO

PROCNO

Date

INIme

PROBHD

PULPROG

TD

SOLVENT

NS
DS
SWH

FIDRES

$\mathrm{AQ}$
$\mathrm{RG}$

RG

DE

TE

D1

FLF160521-ZC -

20161006

21.00

$5 \mathrm{~mm}$ PABBO BB-

$\mathrm{zg} 30$

65536

$\mathrm{CDCl3}$

16
2

$8223.685 \mathrm{~Hz}$

$0.125483 \mathrm{~Hz}$

$3.9846387 \mathrm{sec}$ 203

60.800 usec

6.50 use $298.8 \mathrm{~K}$

1. $000 \odot \odot \odot \odot \odot \mathrm{sec}$

$====$

P1

PL1

PL1W

SF01

SF

WDW

WDW

LB

GB

CHANNEL

1

13.80 usec
$-1.00 \mathrm{~dB}$
$13.18669796 \mathrm{~W}$
$400.1724712 \mathrm{MHz}$
$32768 \mathrm{MHz}$
$400.1700026 \mathrm{MHz}$
$\mathrm{EM}$
$\Theta$
$0.30 \mathrm{~Hz}$
$\odot$
1.00



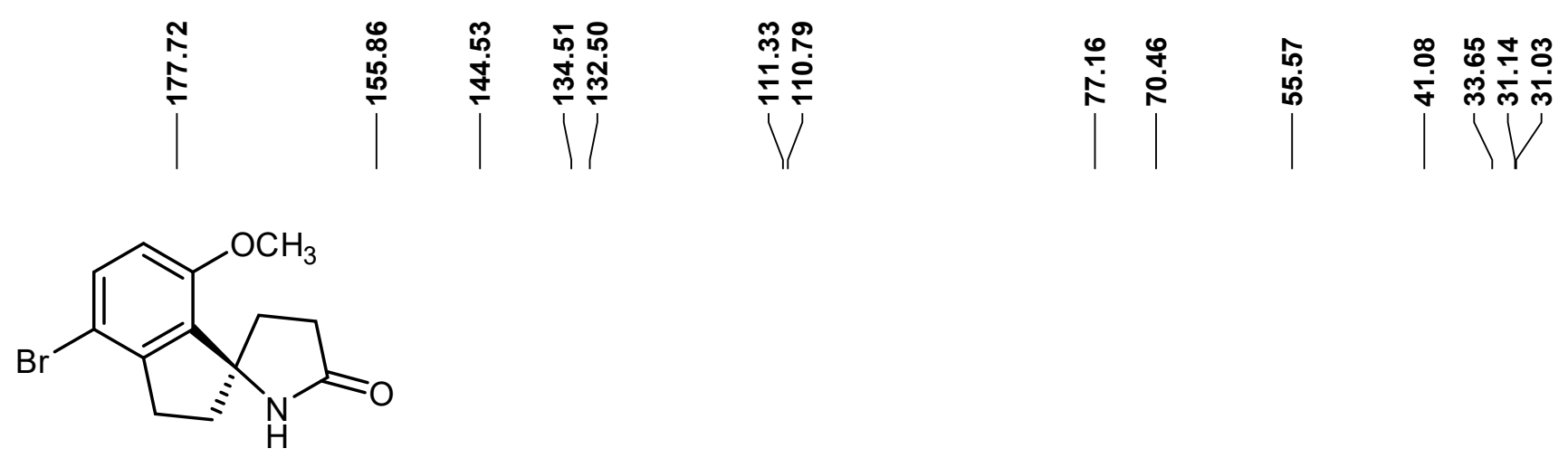

$6 b$

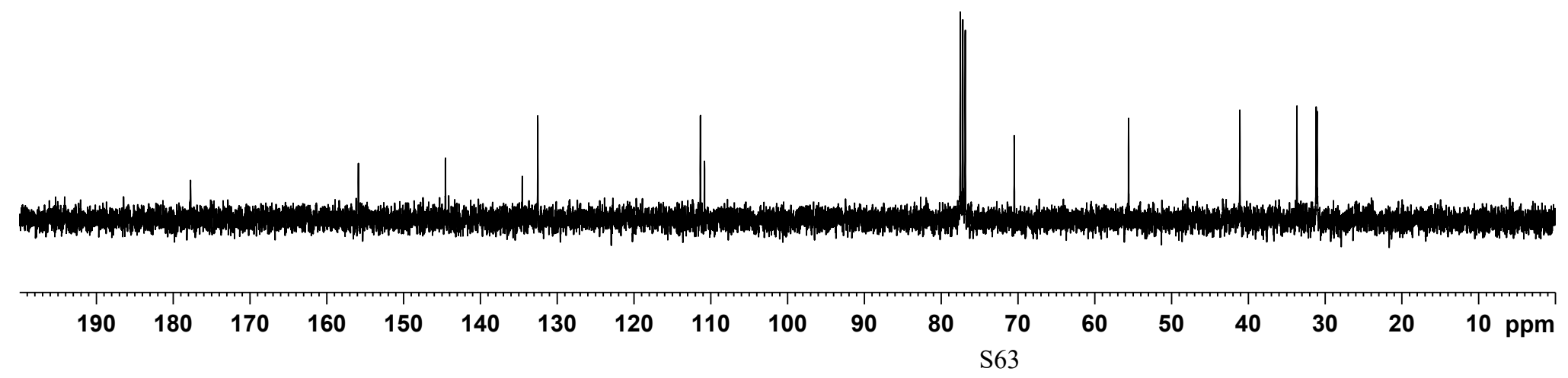

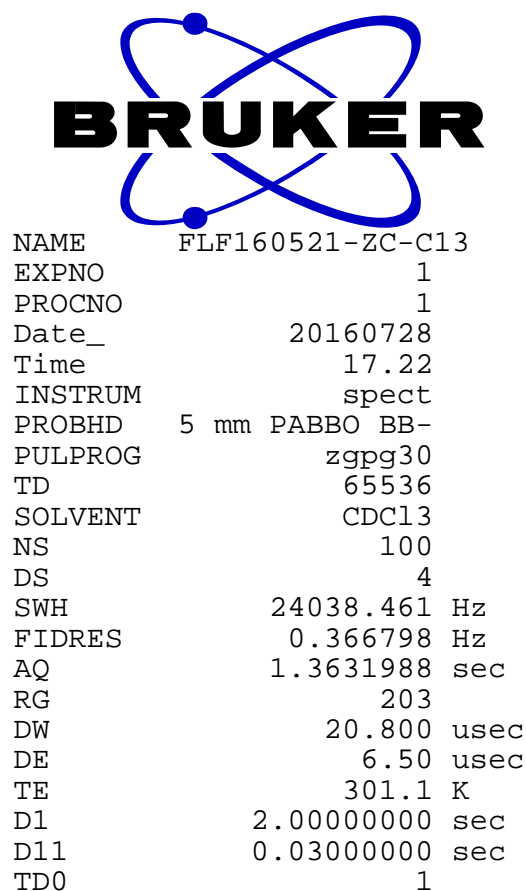

$=======$ CHANNEL $f$ NUC1

PI 1

PL1W

$======$ $13 \mathrm{C}$

$.50 \mathrm{use}$
$.00 \mathrm{~dB}$

SF01

$57.32743073 \mathrm{~W}$

$100.6328888 \mathrm{MHz}$

= ==ニ=== CHANNEL f2 =======

CPDPRG2

$\mathrm{NUC2}$

PCPD2

PL2

PL12

PL2W

PL12W

PL13W

SI

WDW

SSB

LB

PC

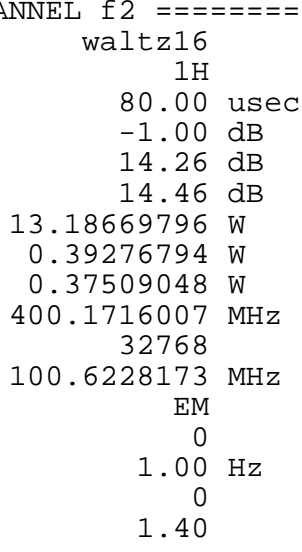




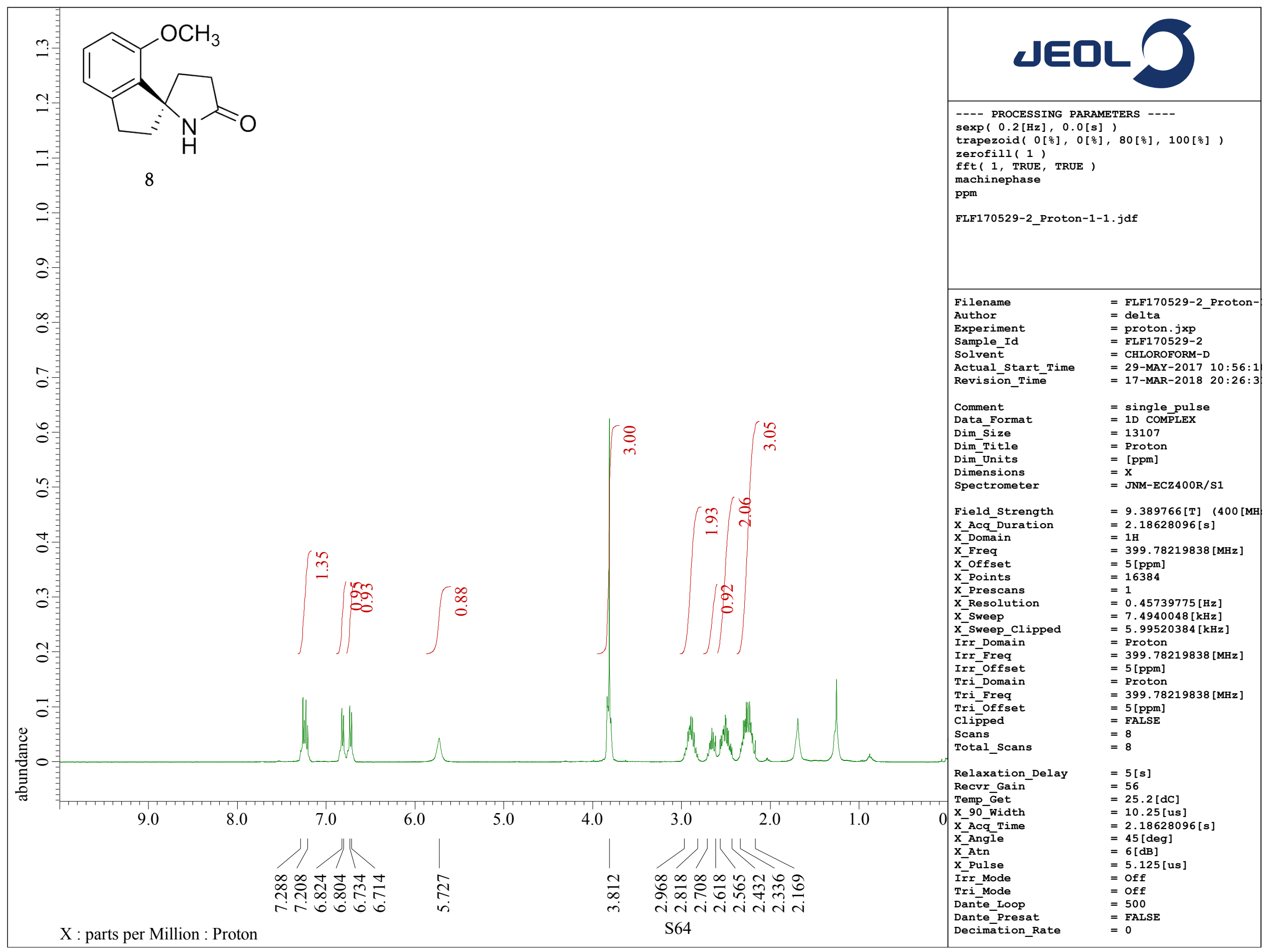




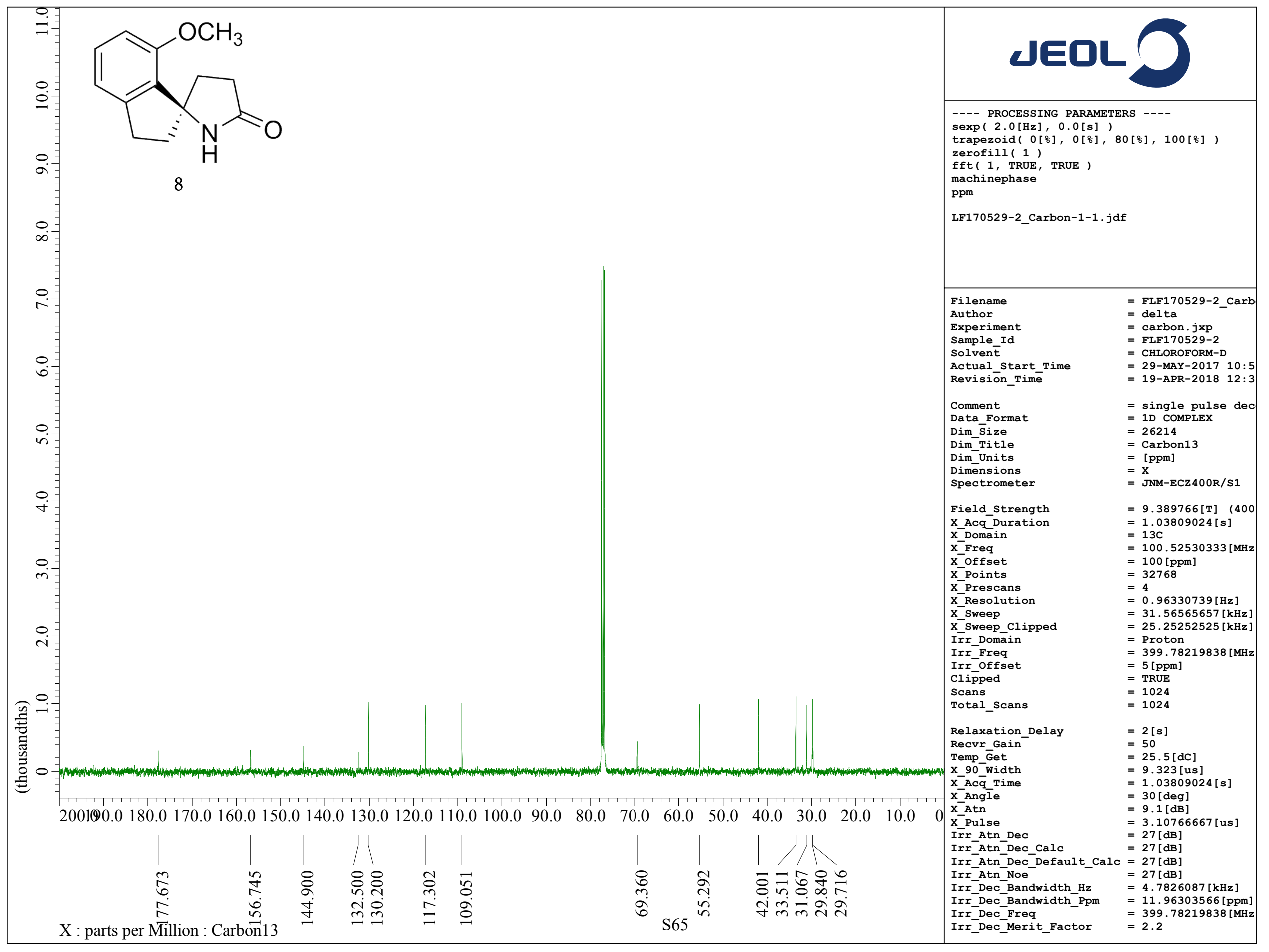




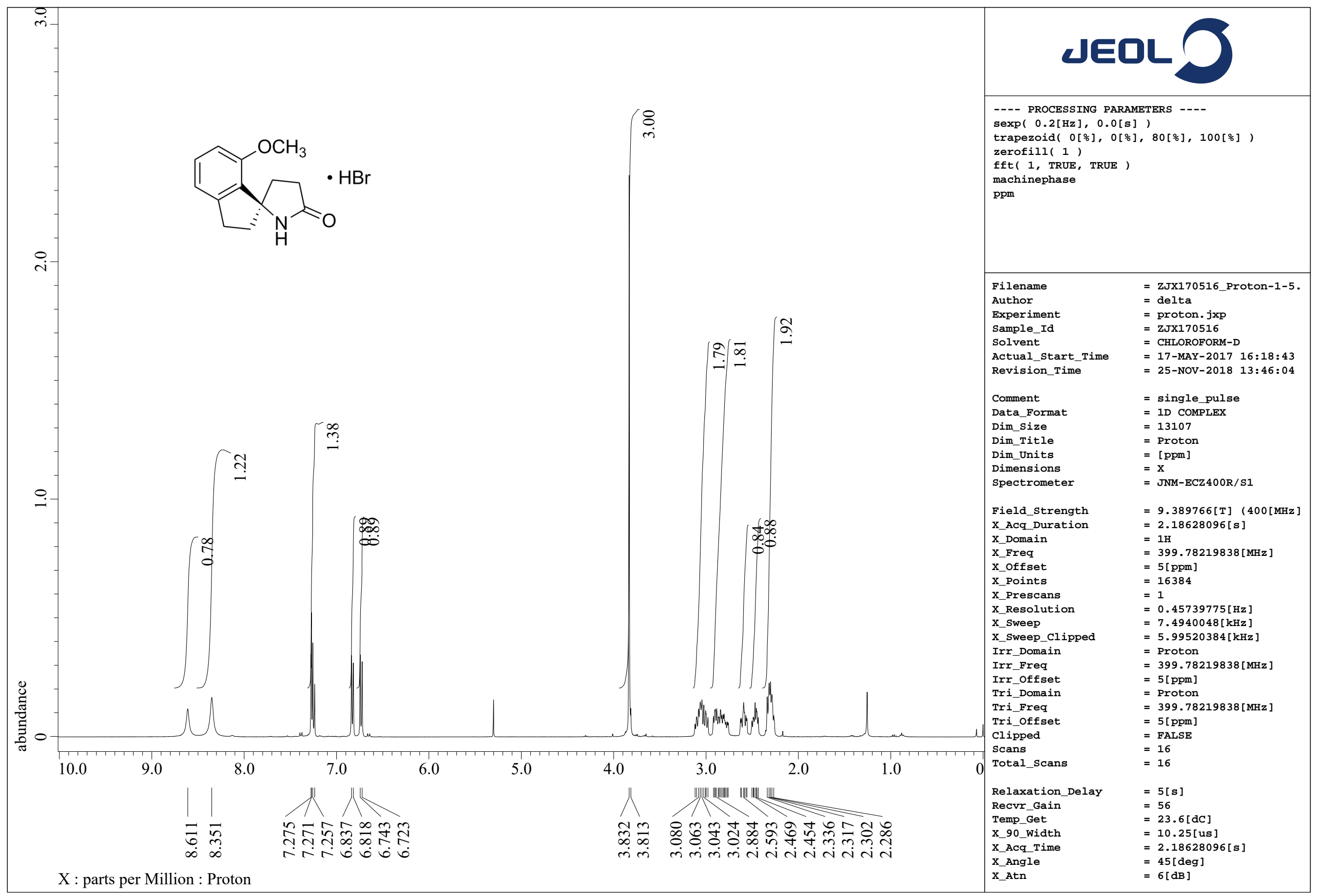




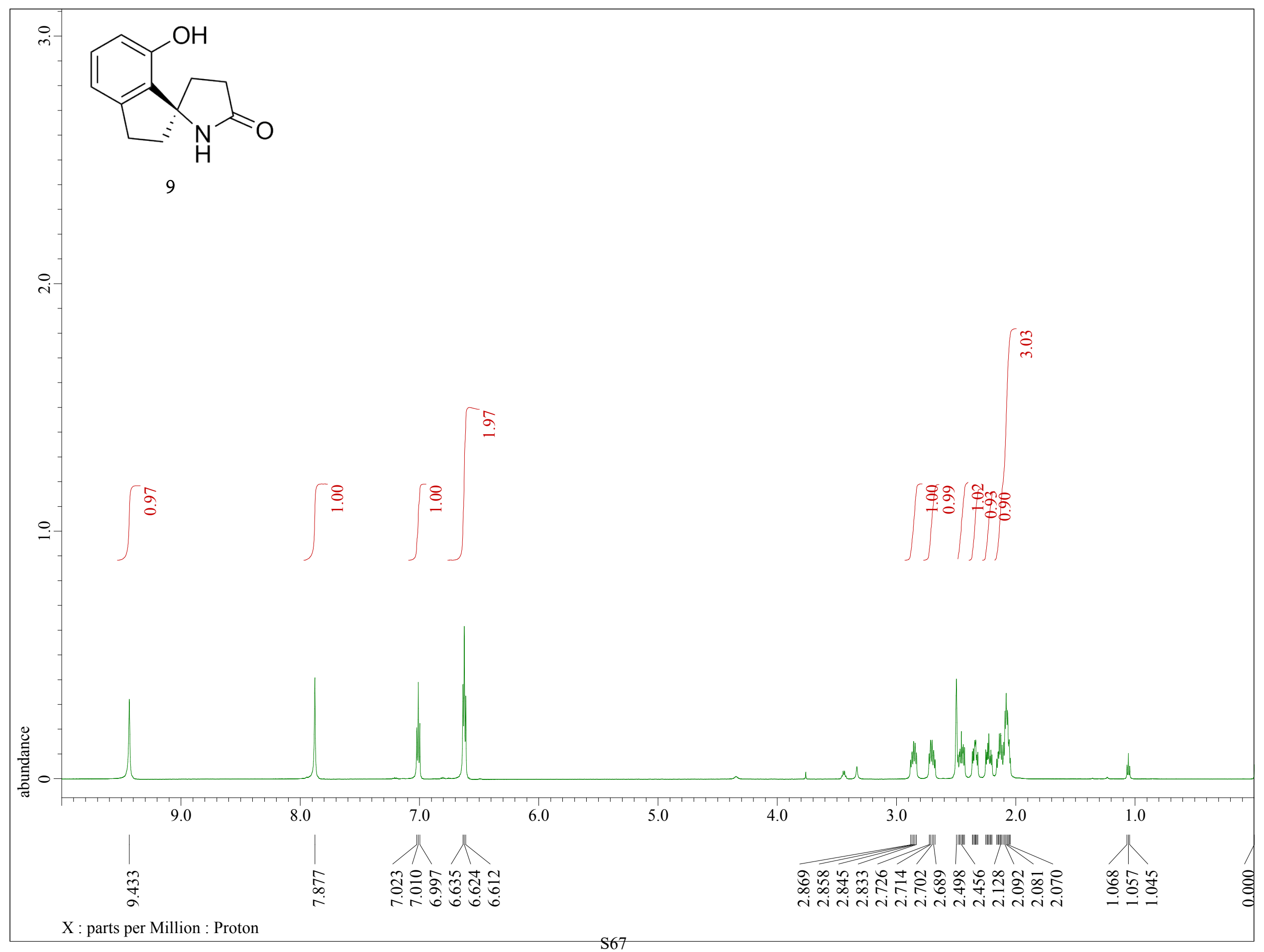




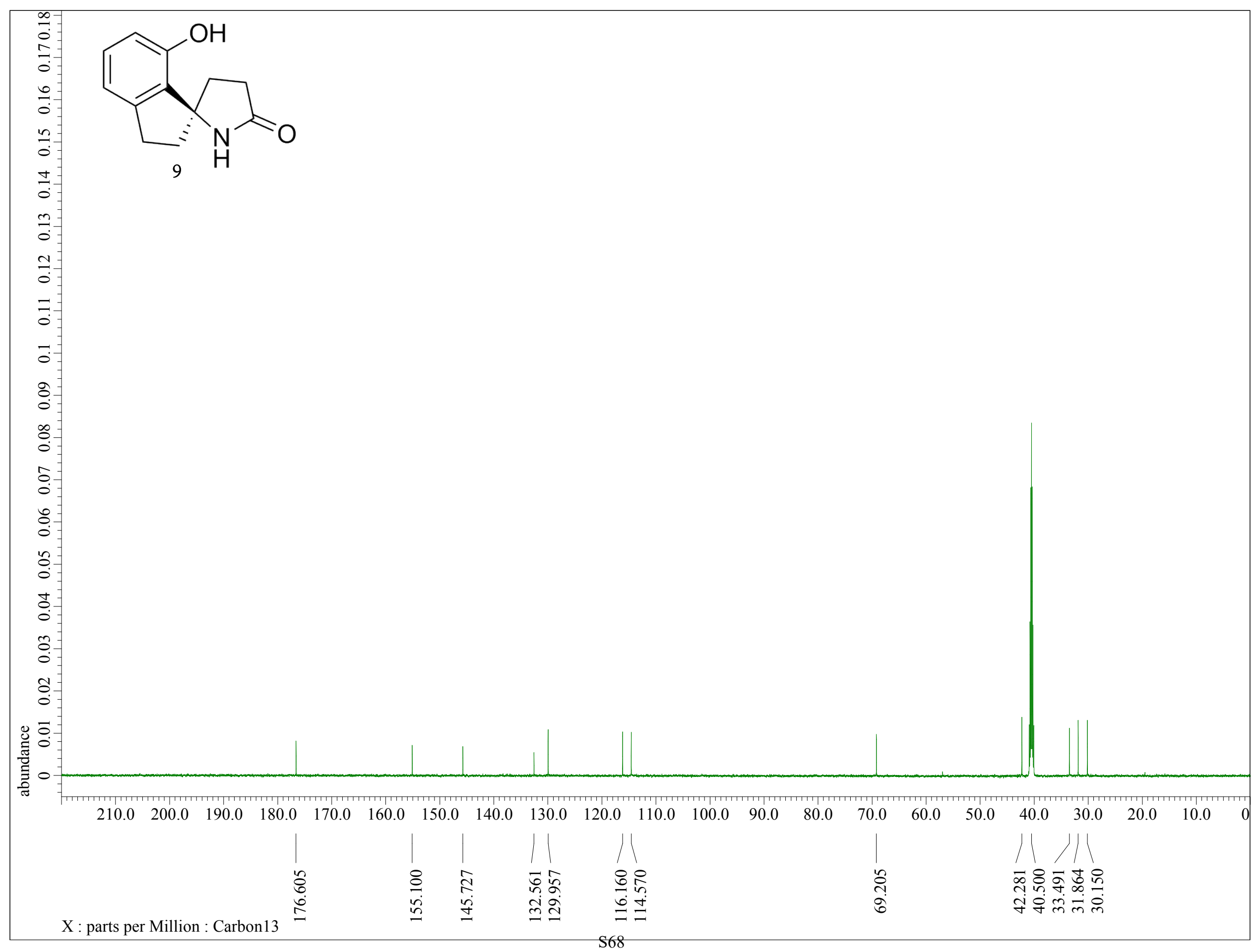




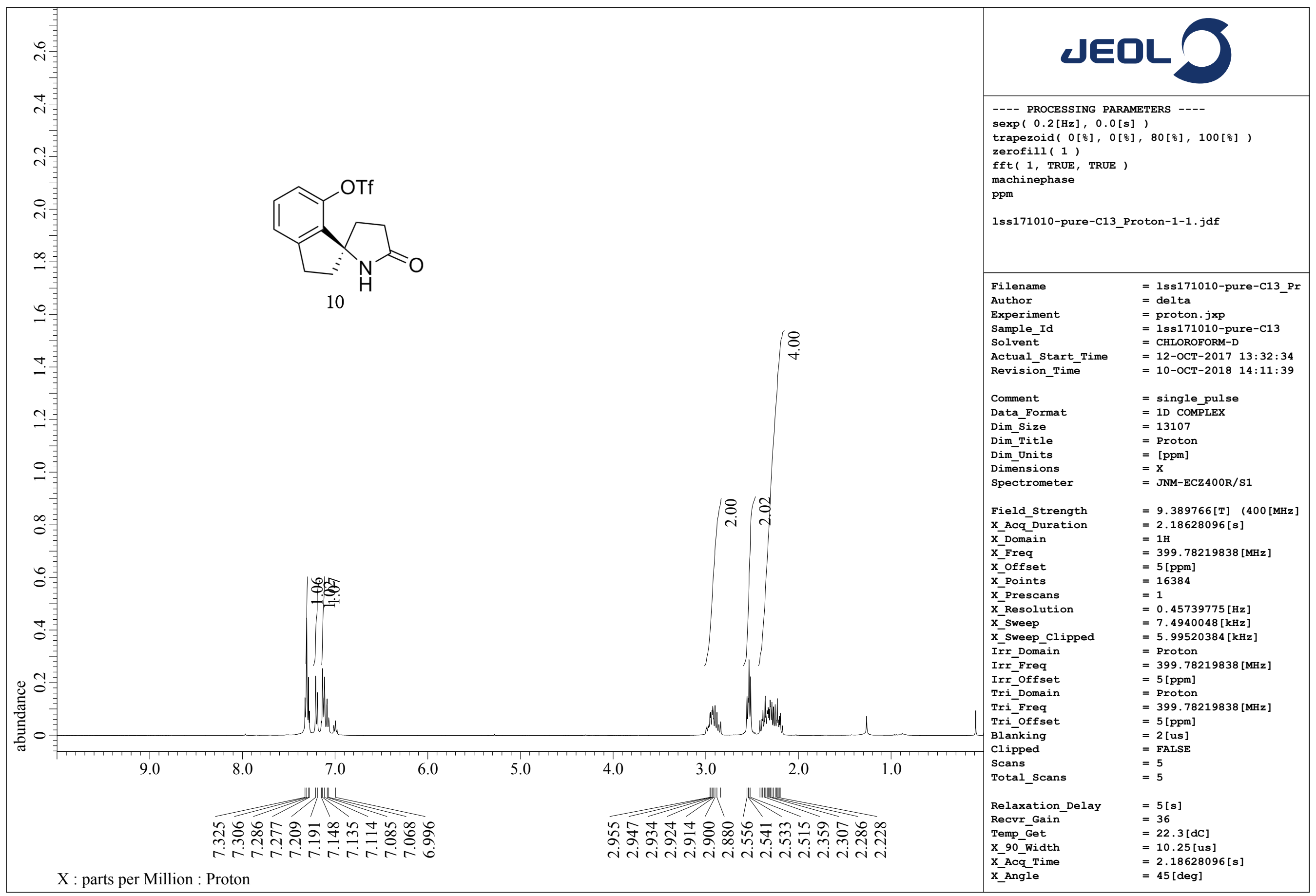




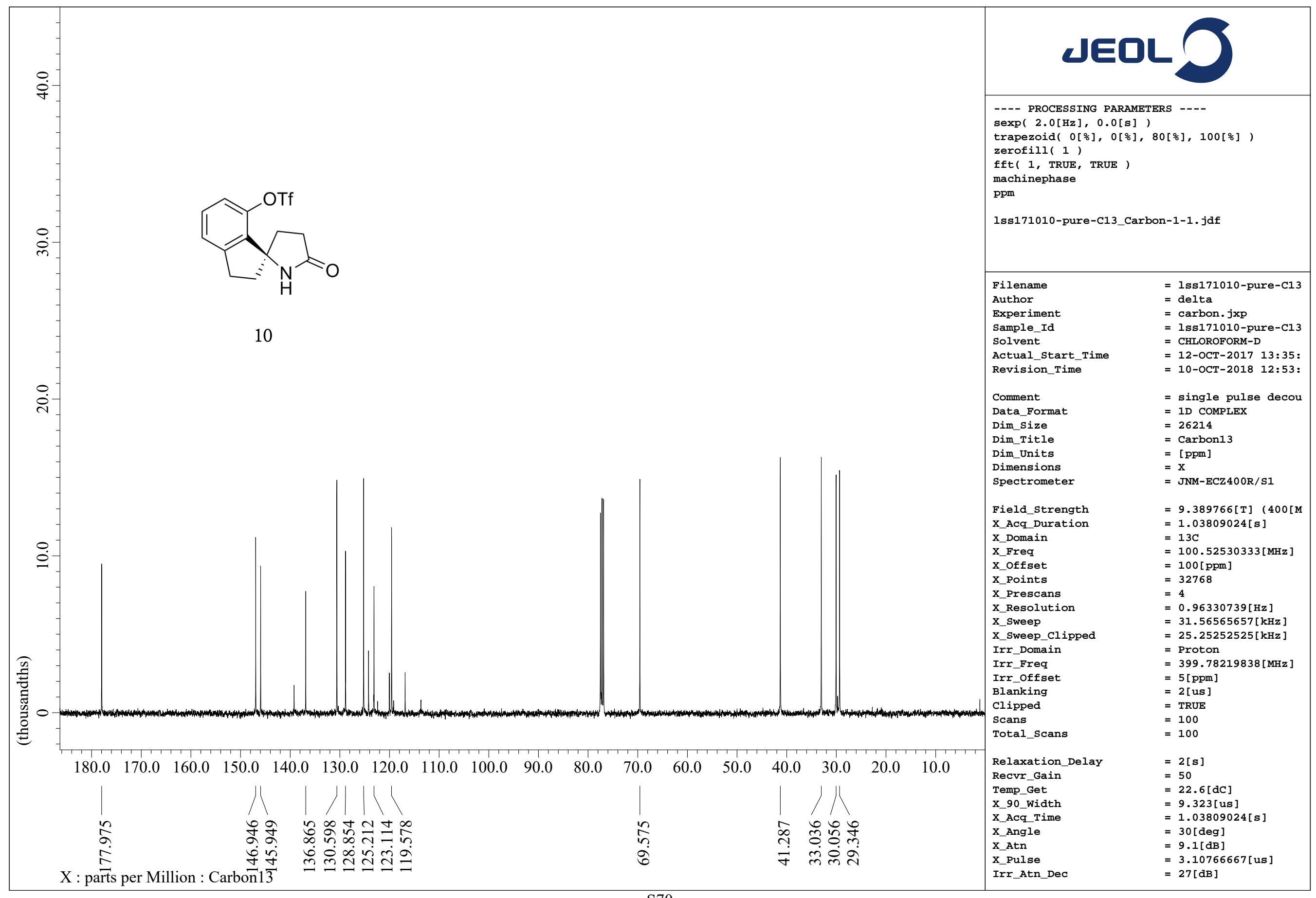



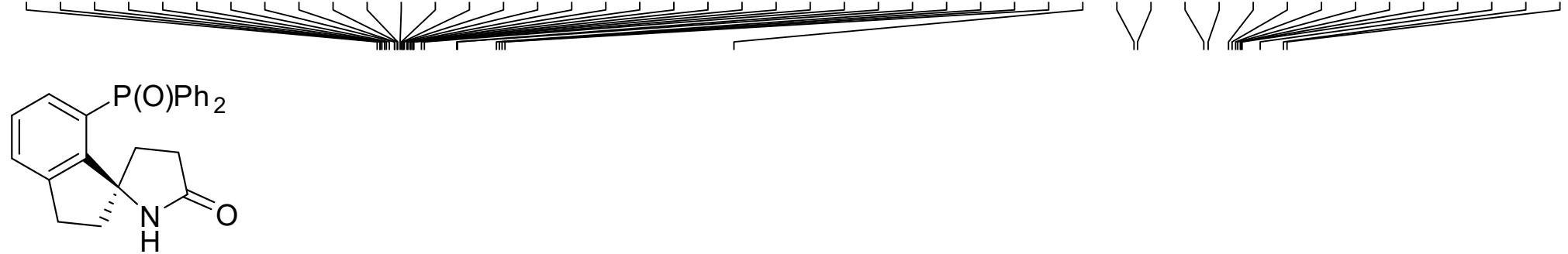

$11 \mathrm{a}$
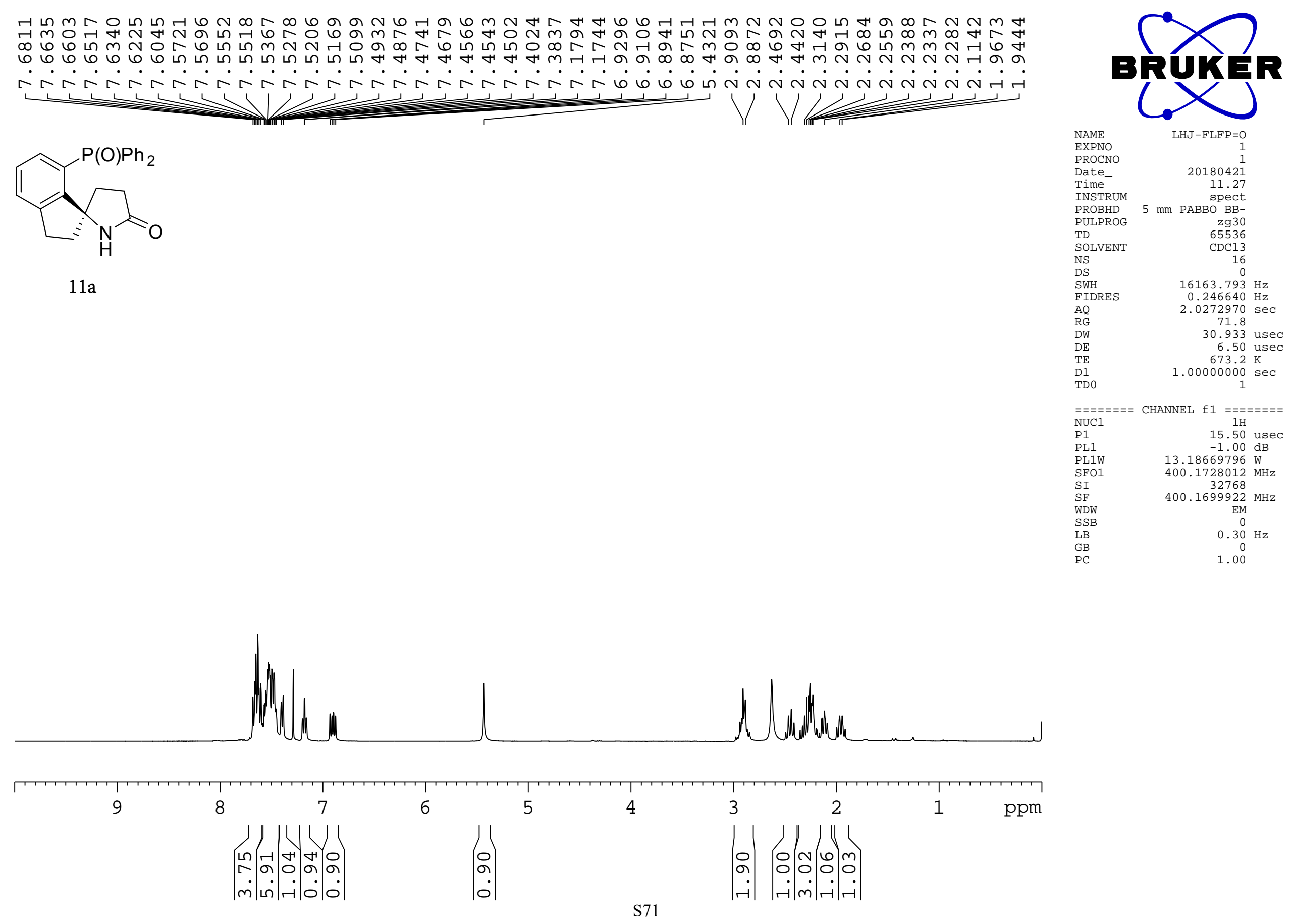


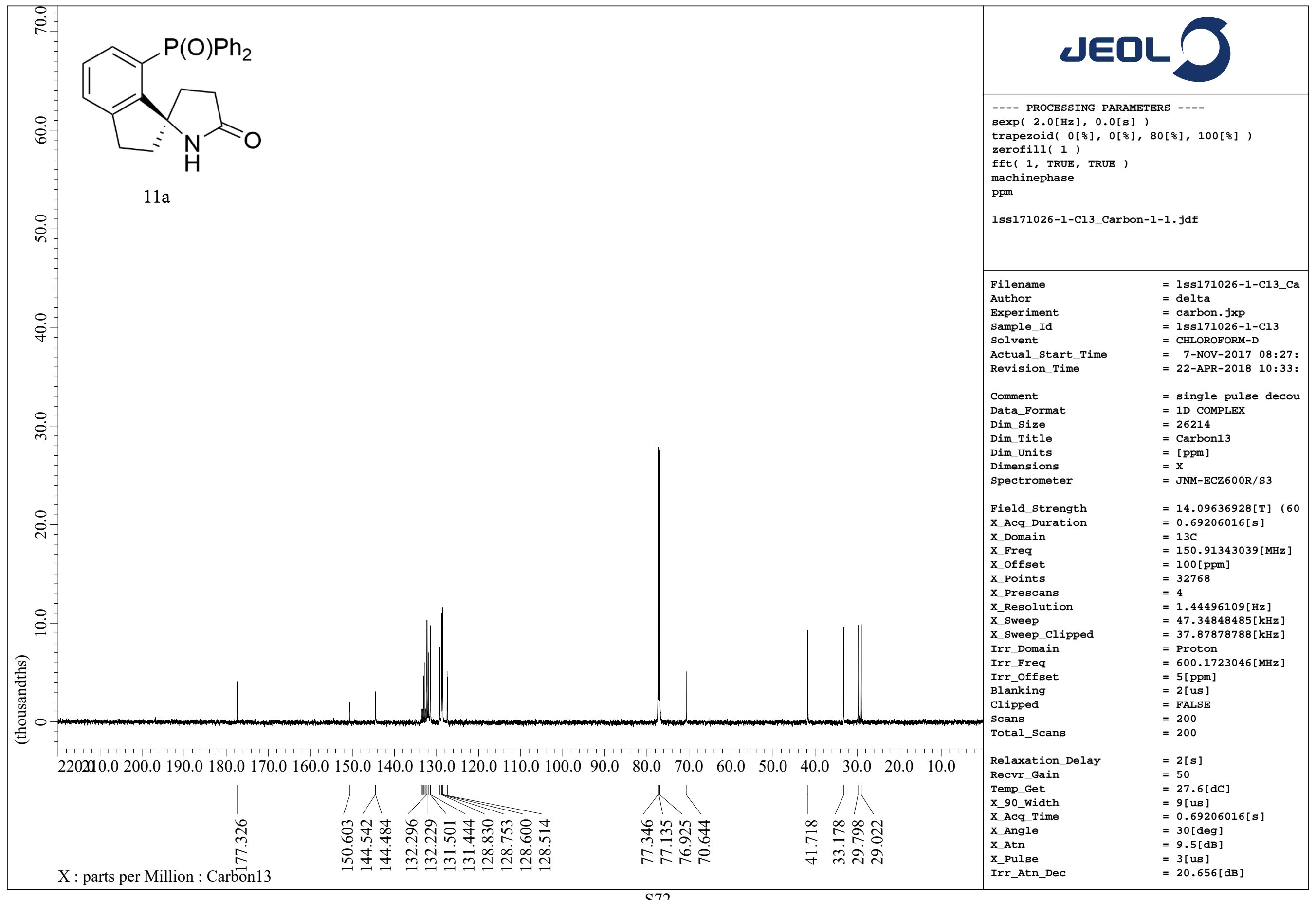




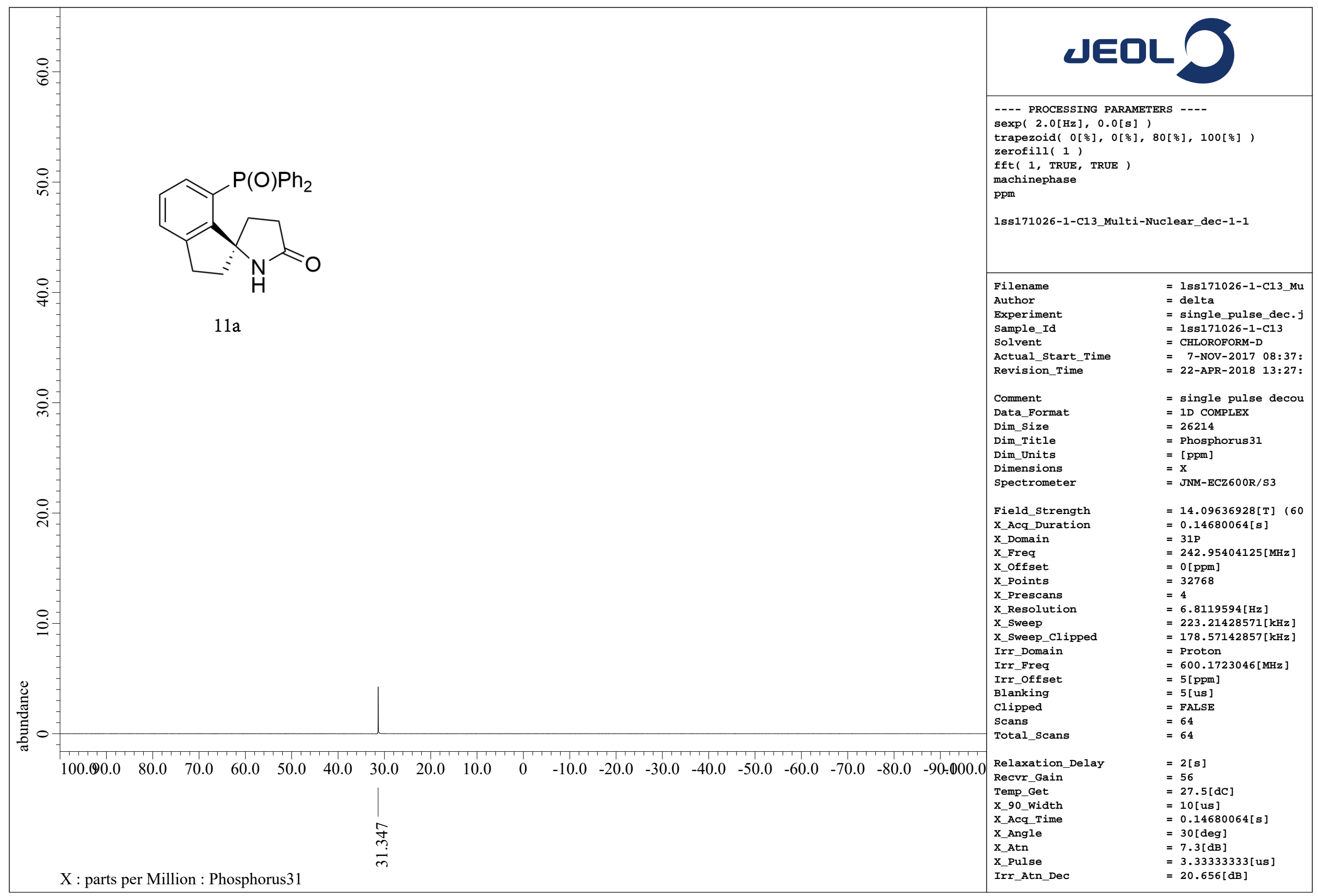




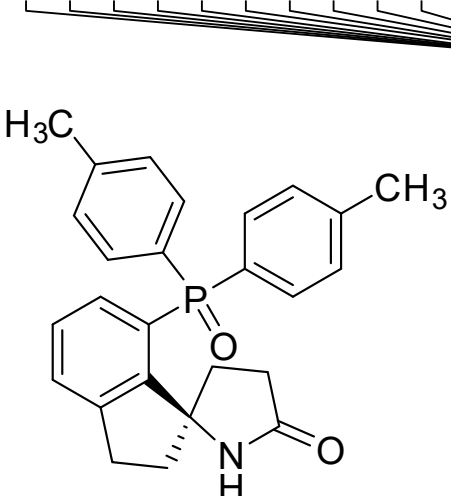

$11 \mathrm{~b}$

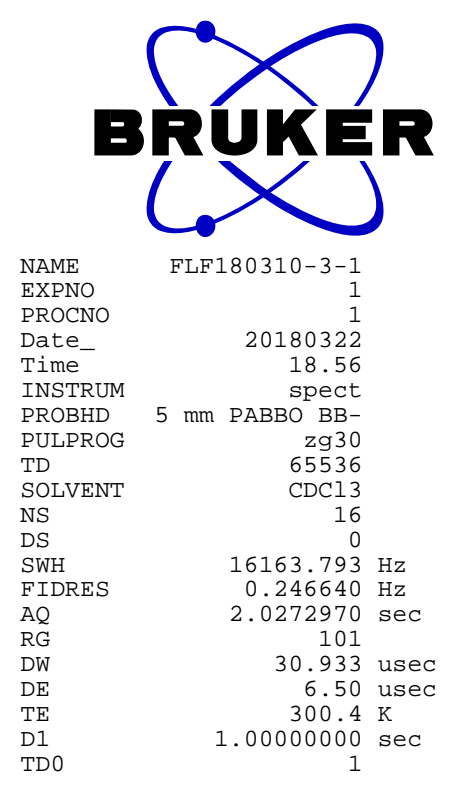

\section{$====$}

NUC1

P1 1
PL1
PLO1

SI

$\mathrm{SF}$

WDW

LB

$\mathrm{PC}$

BRUKER

FLF180310-3-1

ANNEL $f 1$$$
15.50 \text { usec }
$$

$13.18669700 \mathrm{~dB}$

400.1728012 MHZ

$400.1699976 \mathrm{MHZ}$

$\mathrm{EM}$
0
$0.30 \mathrm{~Hz}$

1.00

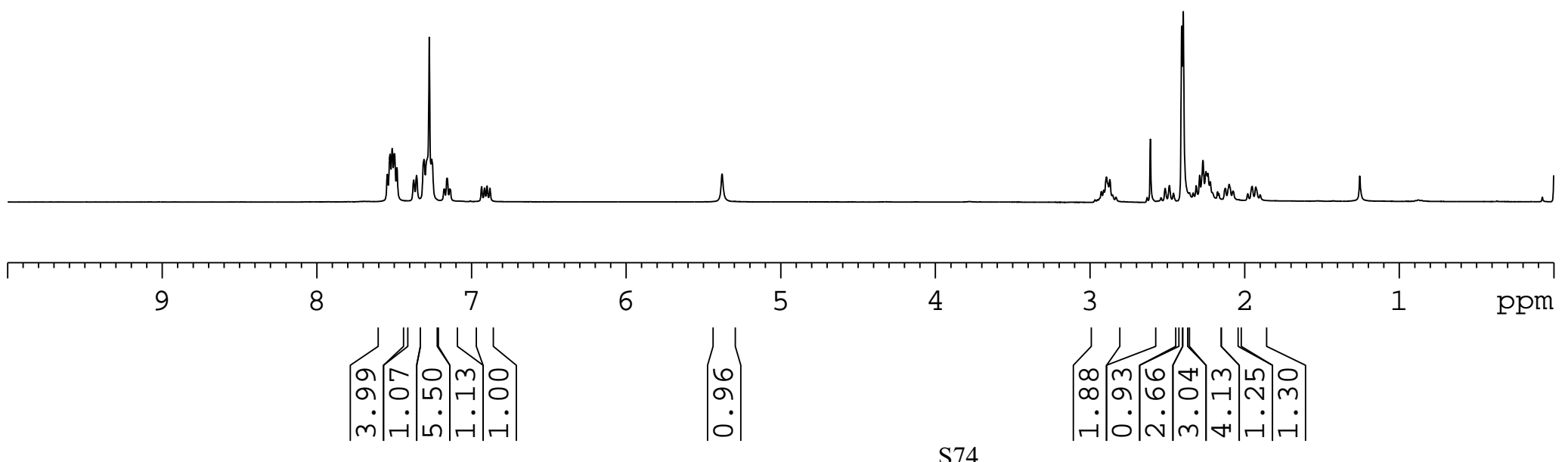


건ㄷำ

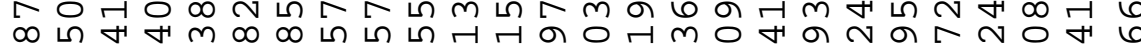
ㄱ.

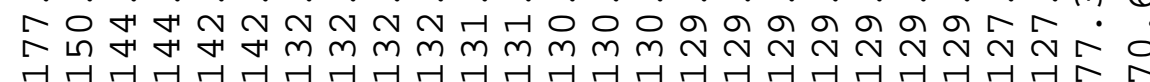
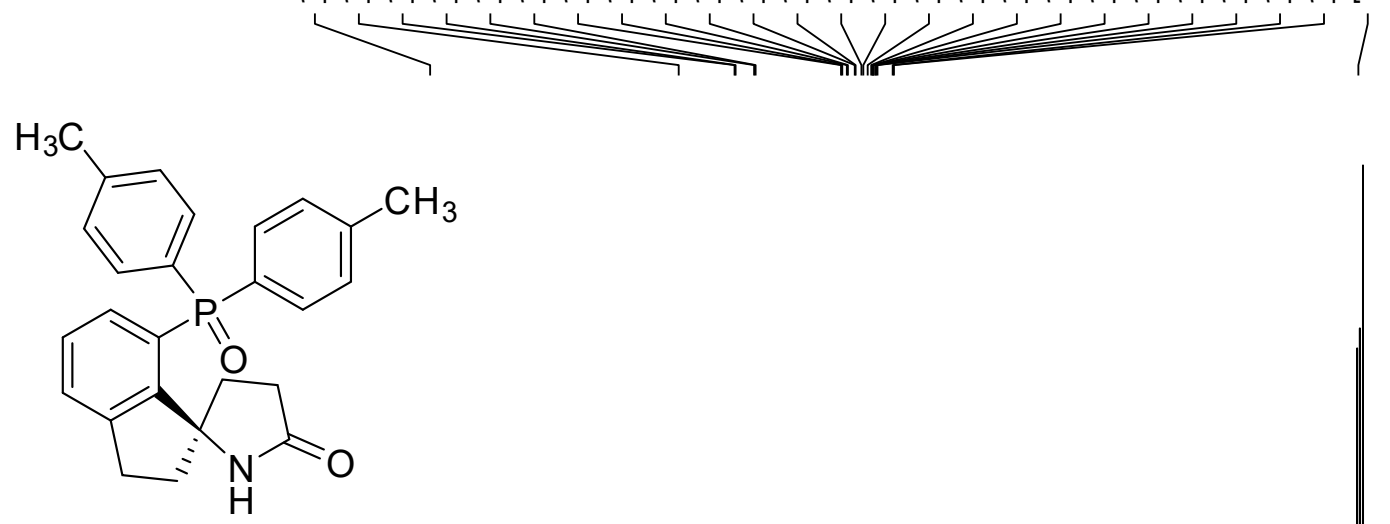

$11 \mathrm{~b}$

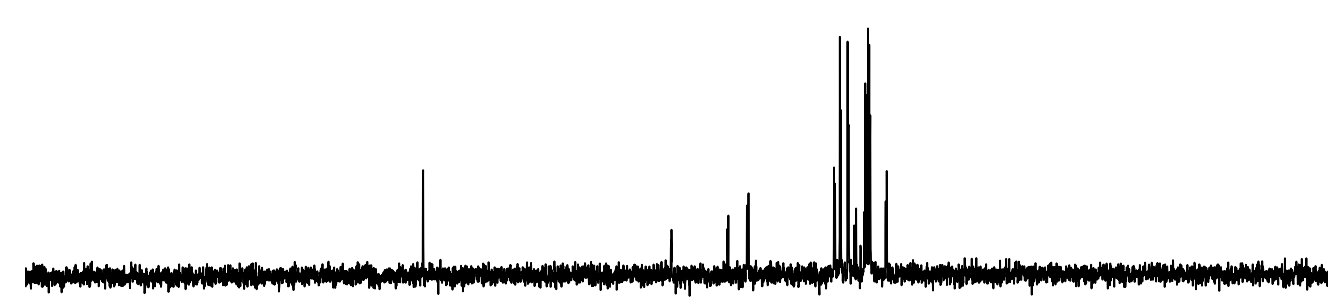

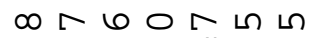
m๑m

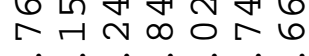

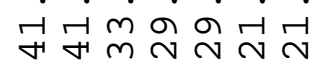
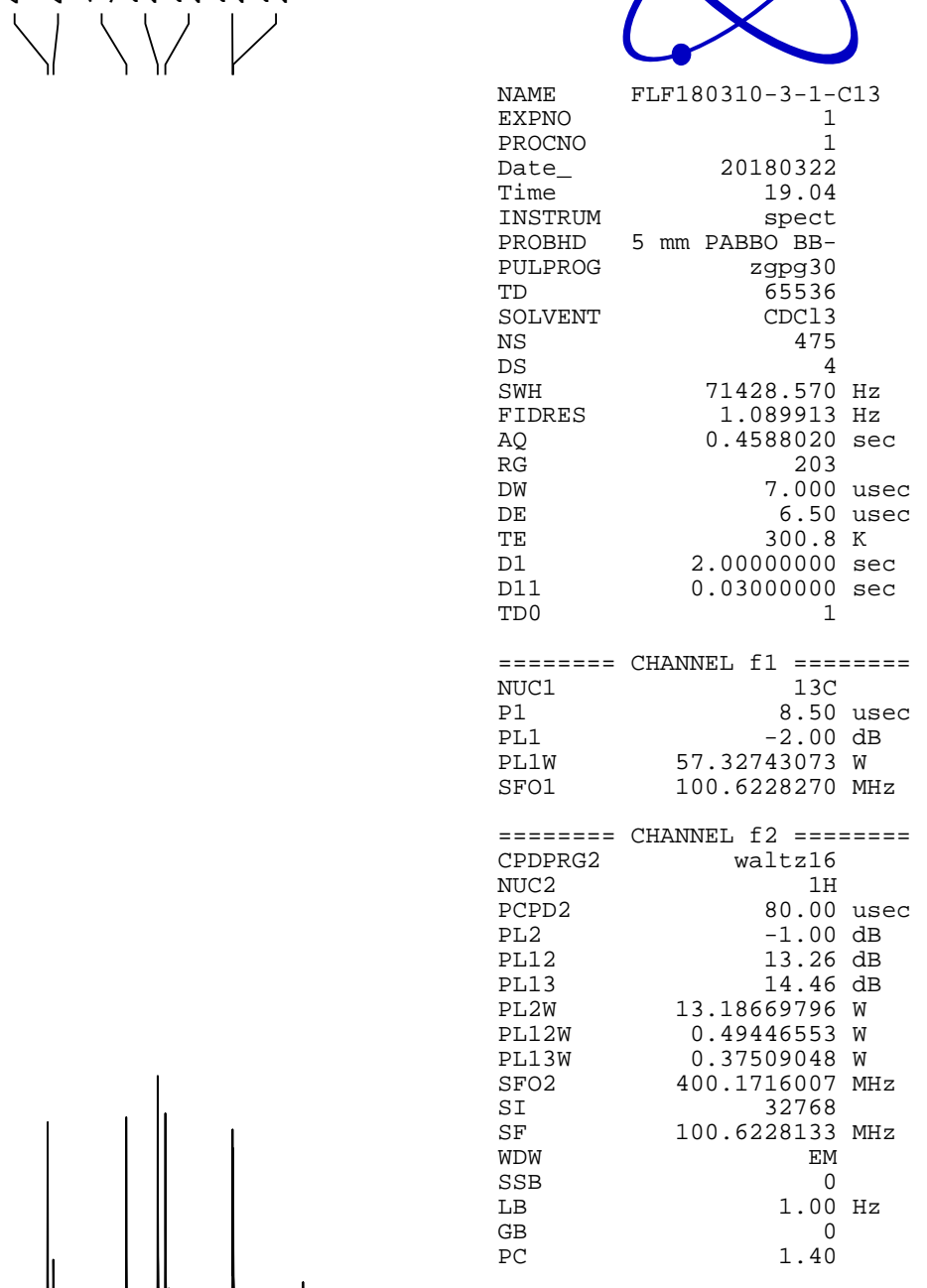

BRUKER $(><)$

SOLVENT

DS

SWH

AQ

DE

D1

TDO

$====$

PL1

SF01

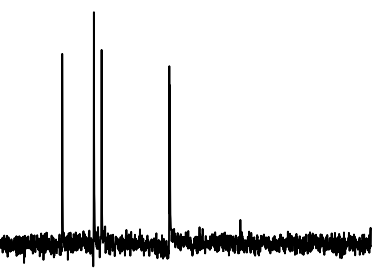

1.40 


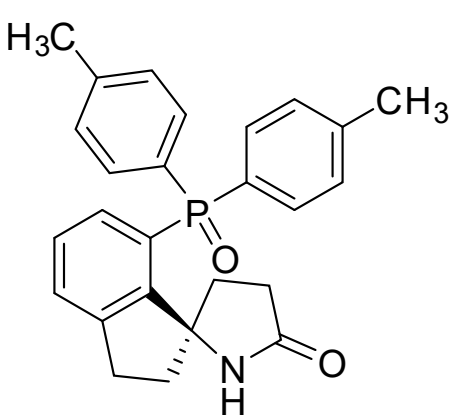

$11 \mathrm{~b}$

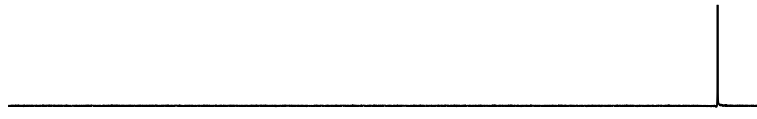

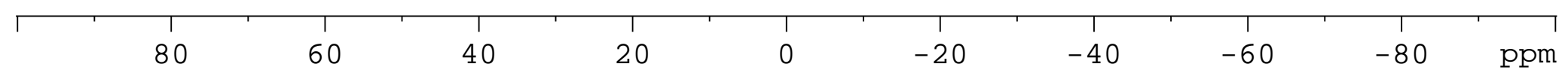



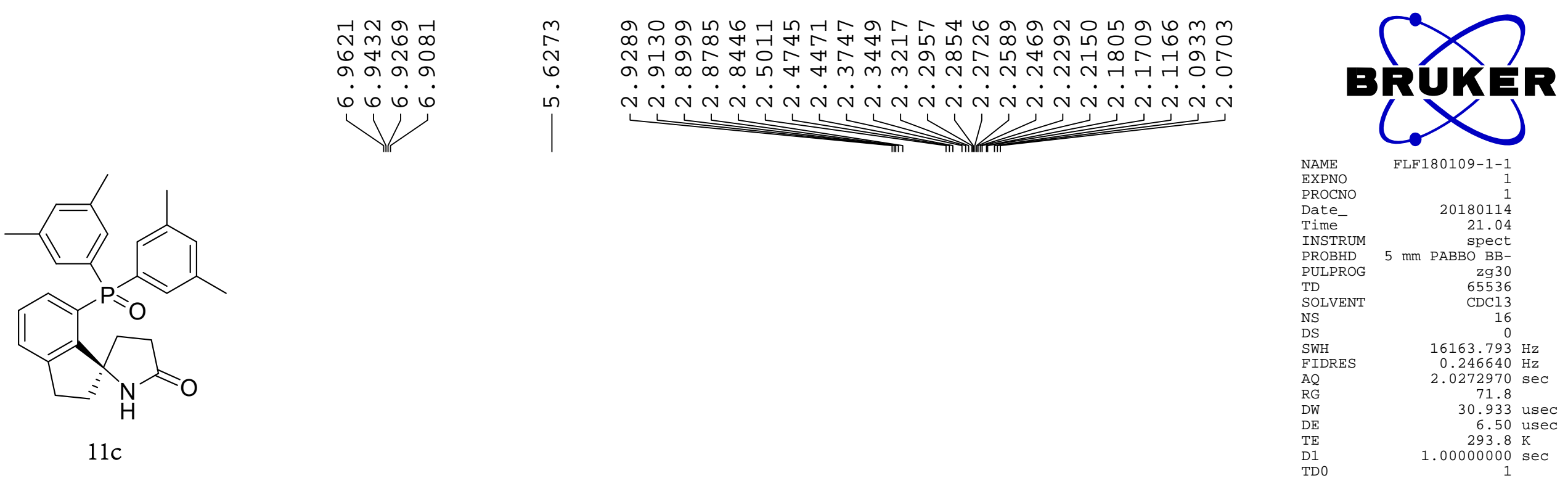

11c

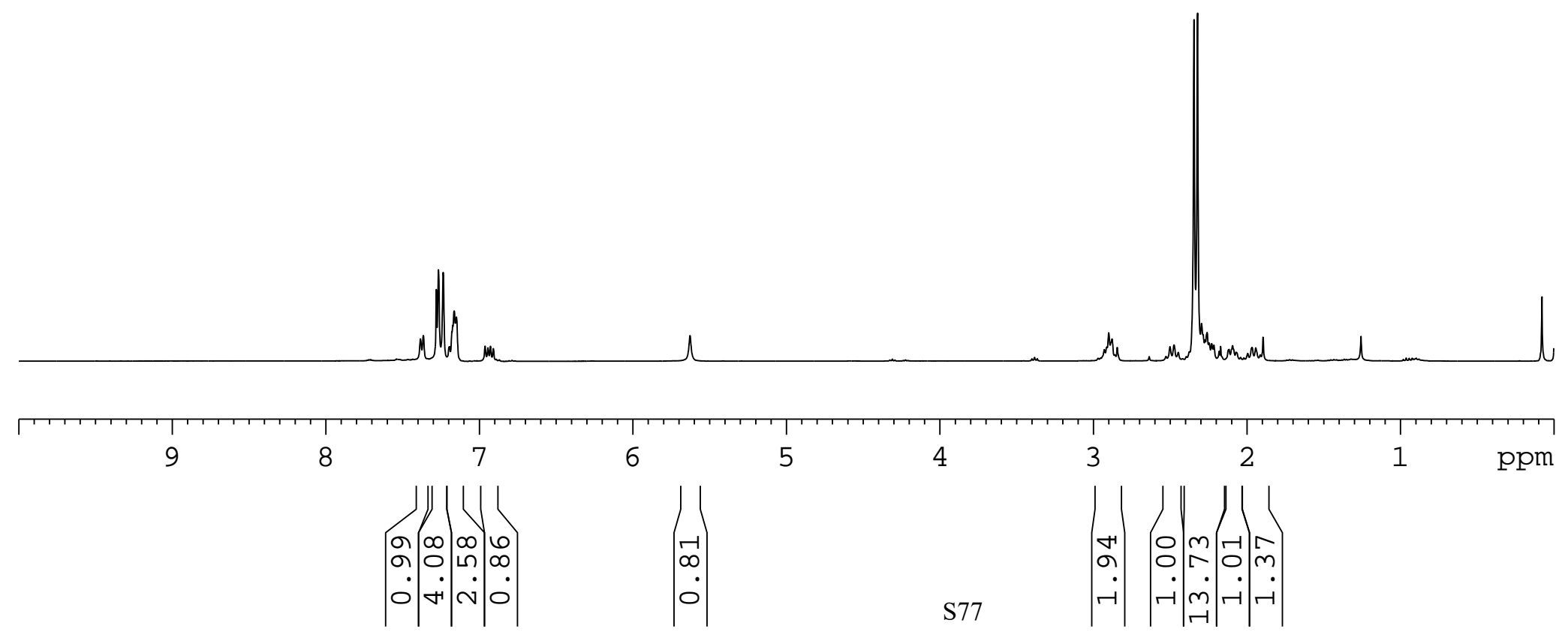


№

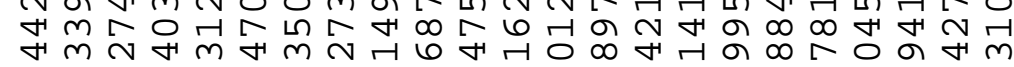

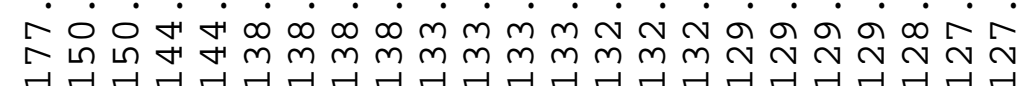

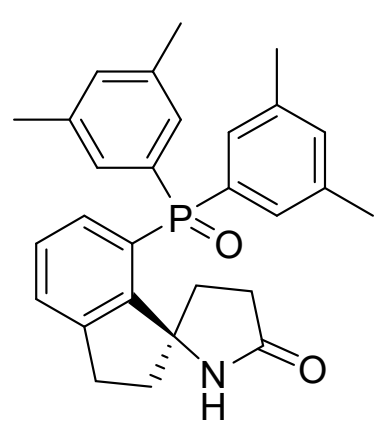

$11 \mathrm{c}$
๑

ヘ のֻ

글
-RUKER $C \times$

NAME

EXPNO

Time-

INSTRUM

PROBHD

TD

SOLVENT

NS

SWH

FIDR

$\mathrm{AQ}$
$\mathrm{RG}$

DW

TE

D1
D11
TDQ

$====$
NUC1

P1
PL1

SF01

$======$

CPDPR
NUC2

PCPD2

PL2

PL13

PL12W

PL13W

SI

$\mathrm{SF}$

WDW

LB

$\mathrm{PB}$
FLF180109-1-1-C13

20180114

21.14
spect

$\mathrm{mm}$ PABBO BB

zgpg30

$\mathrm{CDCl} 3$

4
71428.570 $1.089913 \mathrm{~Hz}$

7. .000 usec

6.50 usec

$2.00004 .4 \mathrm{k}$

$2.00000000 \mathrm{sec}$
$0.03000000 \mathrm{sec}$

$=$ CHANNEL

$======$

$-2.00$ $57.32743073 \mathrm{~W}$ 100.6228270 MHz

CHANNEL f2 ======== waltz16

80.00 usec

$-1.00 \mathrm{~dB}$
$13.26 \mathrm{~dB}$

$14.46 \mathrm{~dB}$

$13.18669796 \mathrm{~W}$

. $49446553 \mathrm{~W}$

$400.1716007 \mathrm{MHz}$

$100.6228177 \mathrm{MHZ}$ EM

1. $00 \mathrm{~Hz}$

$1.4 \odot$ 


NAME




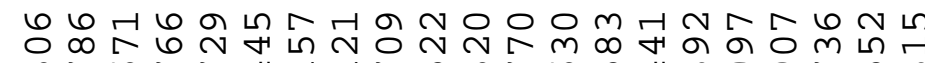
œ ס

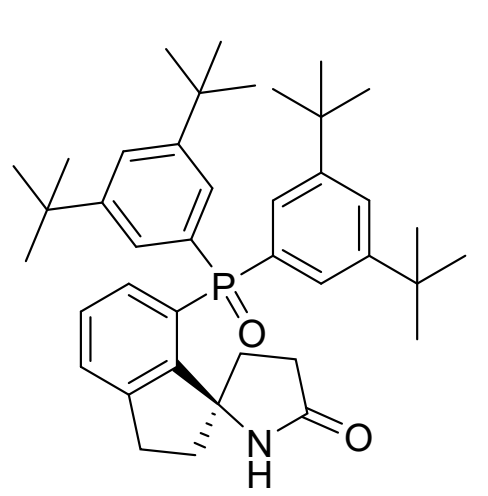

$11 \mathrm{~d}$ $\infty \omega \odot \sim m \infty m \nabla N \omega \forall m \odot \varphi m \infty m \sim N \omega \sim$ №

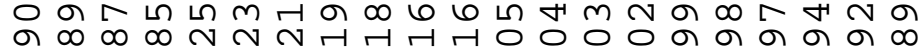
N

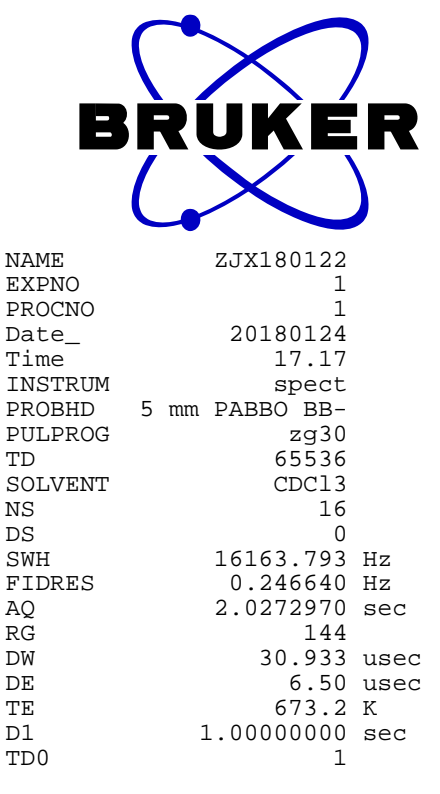

.

NUC1

P1 1

PL1W

SF01

SF

SSB

GB

BRUKER

ZJX180122

0124

pect

DCl3

$6163.793 \mathrm{~Hz}$ $0272970 \mathrm{sec}$

.933 usec $73.2 \mathrm{~K}$

1

$$
\begin{array}{r}
1 \mathrm{H} \\
15.50 \text { usec } \\
-1.00 \mathrm{~dB} \\
13.18669796 \mathrm{~W} \\
400.1728012 \mathrm{MHz} \\
32768 \\
400.1700055 \mathrm{MHz} \\
\mathrm{EM} \\
0 \\
0.30 \mathrm{~Hz} \\
0 \\
1.00
\end{array}
$$

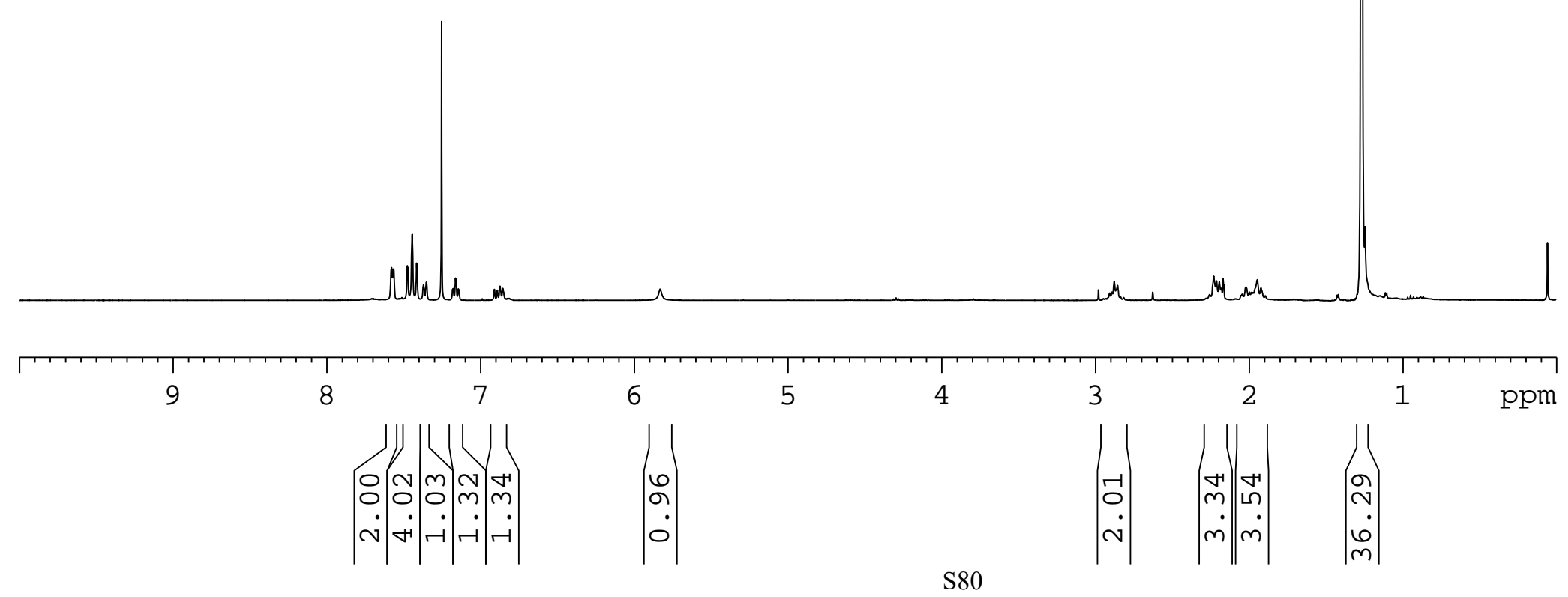


ஓ NNm N

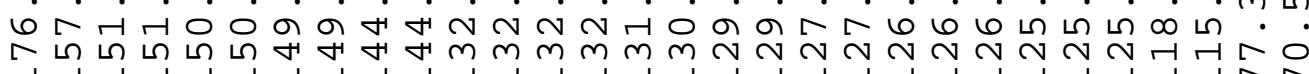

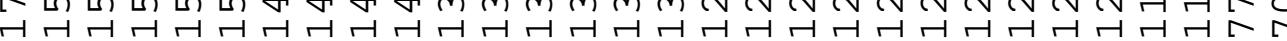

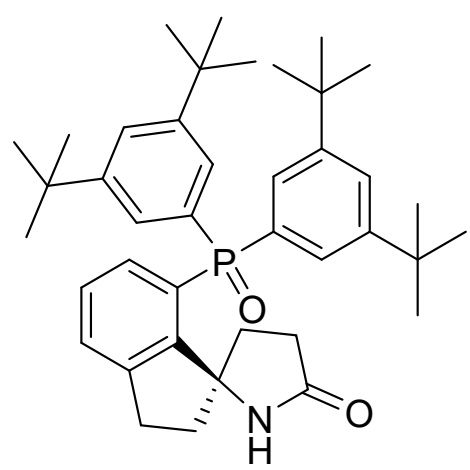

$11 \mathrm{~d}$ $\infty$ H $0, \pi m+1$

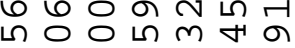
귕

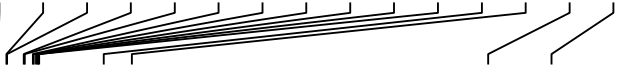

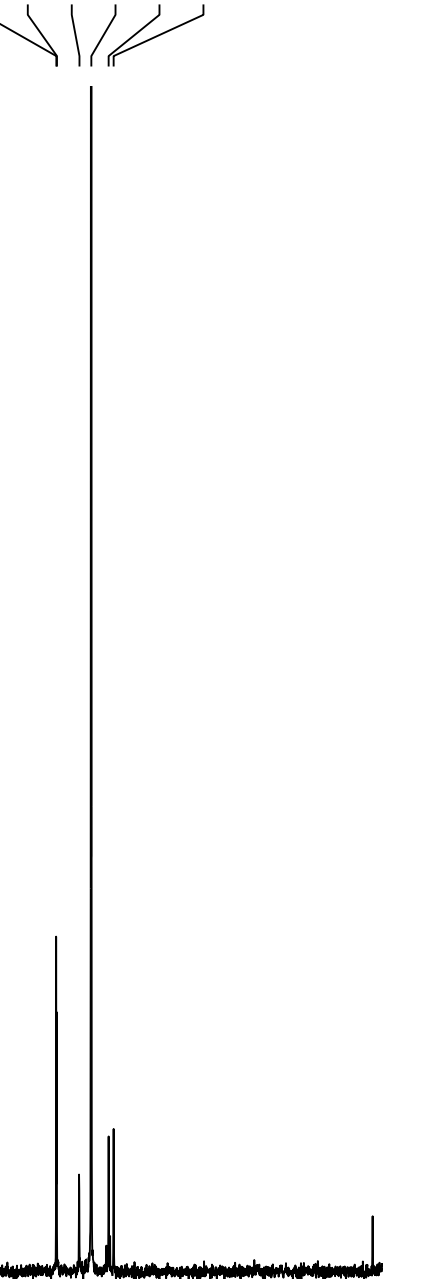




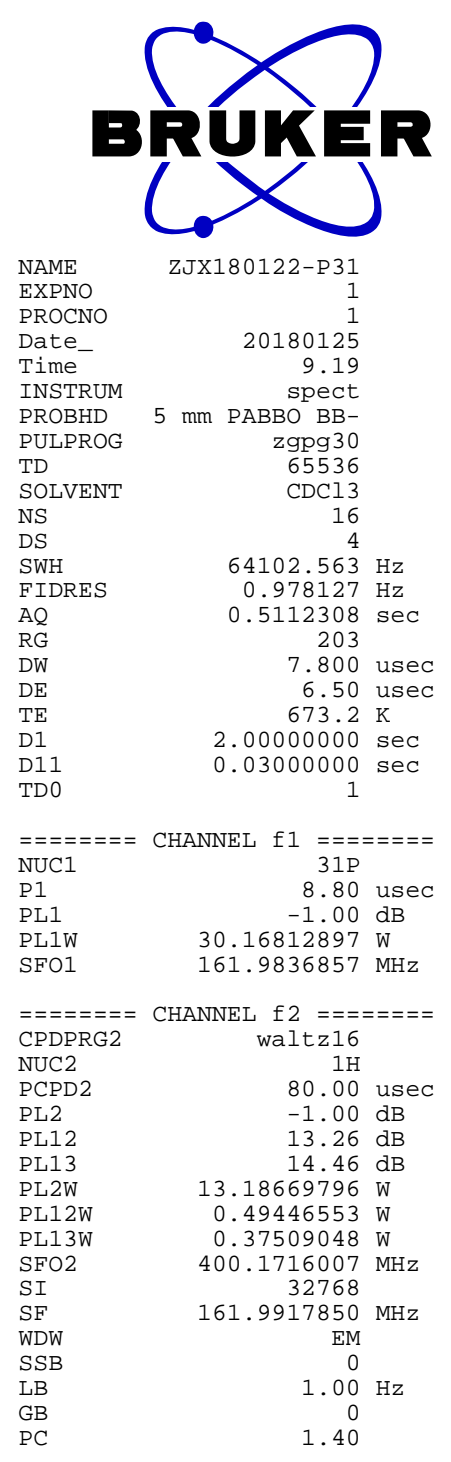




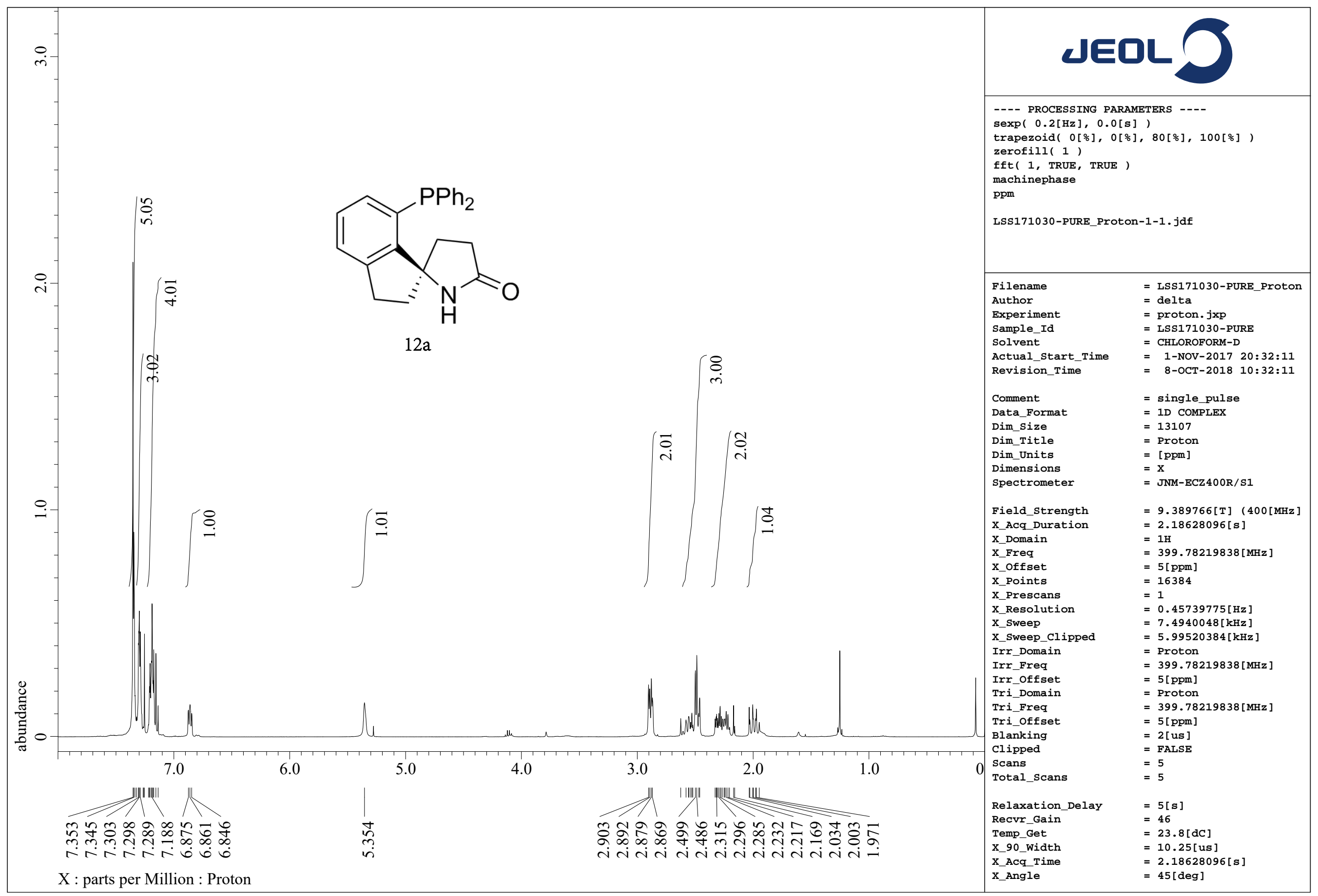




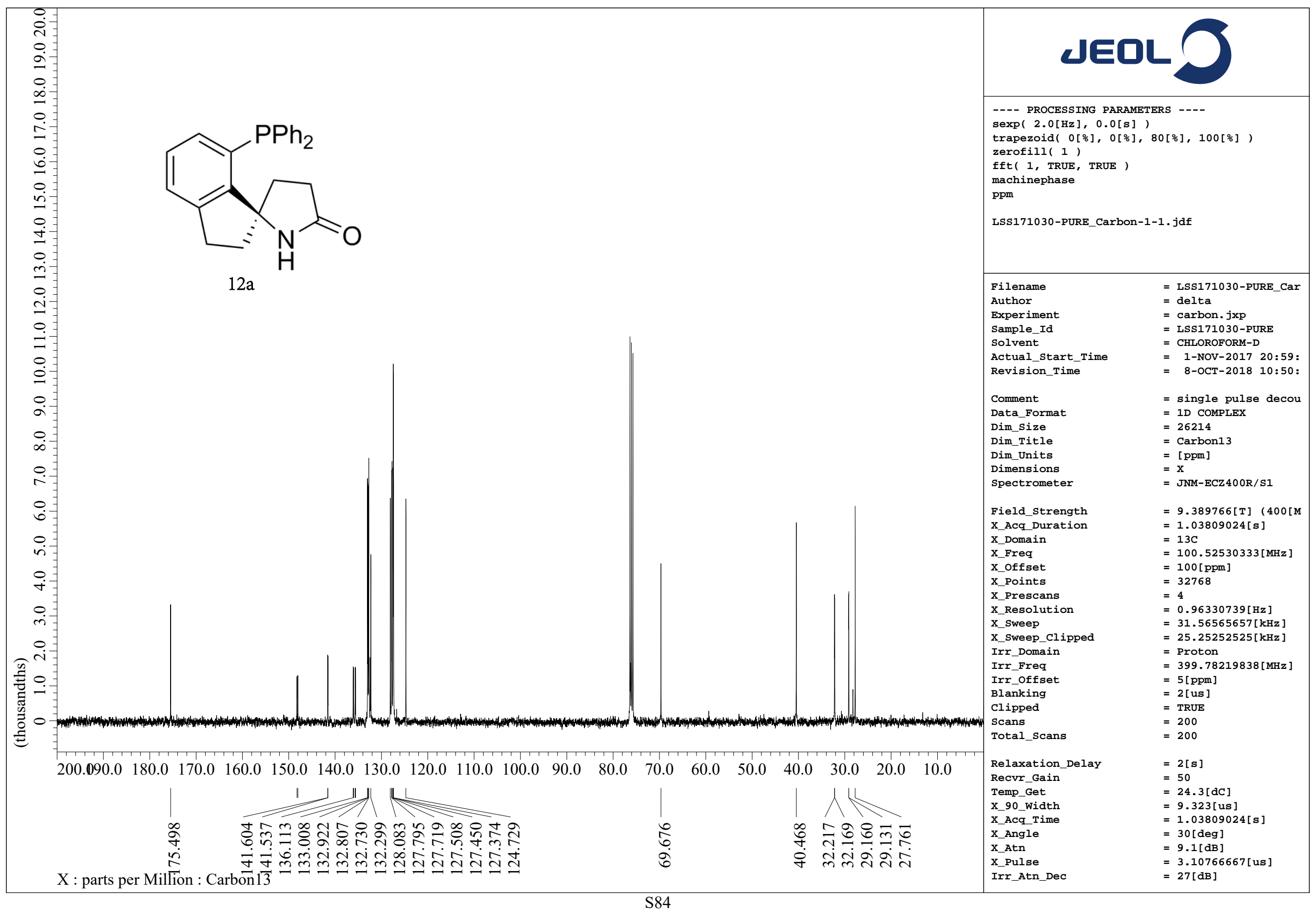




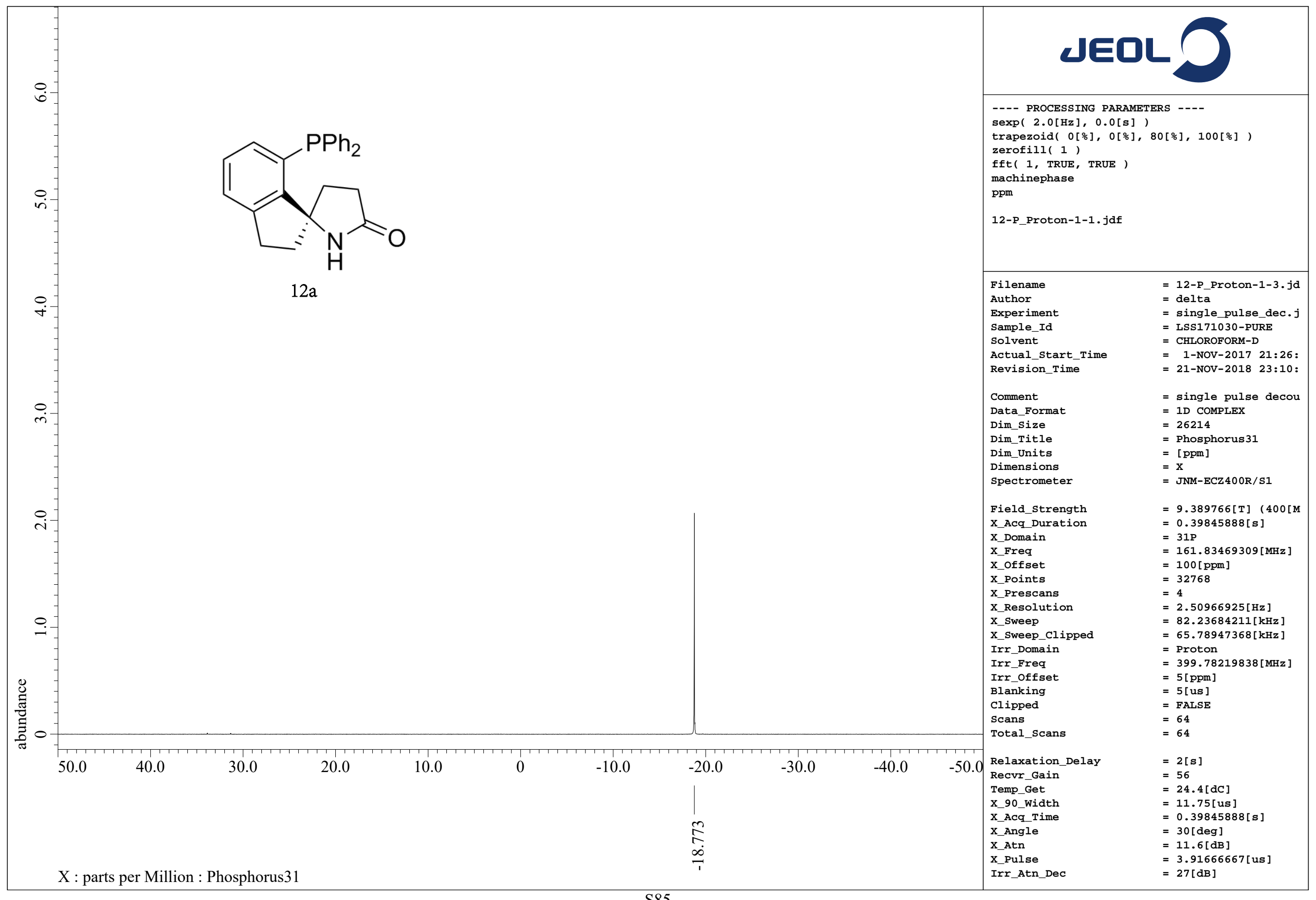




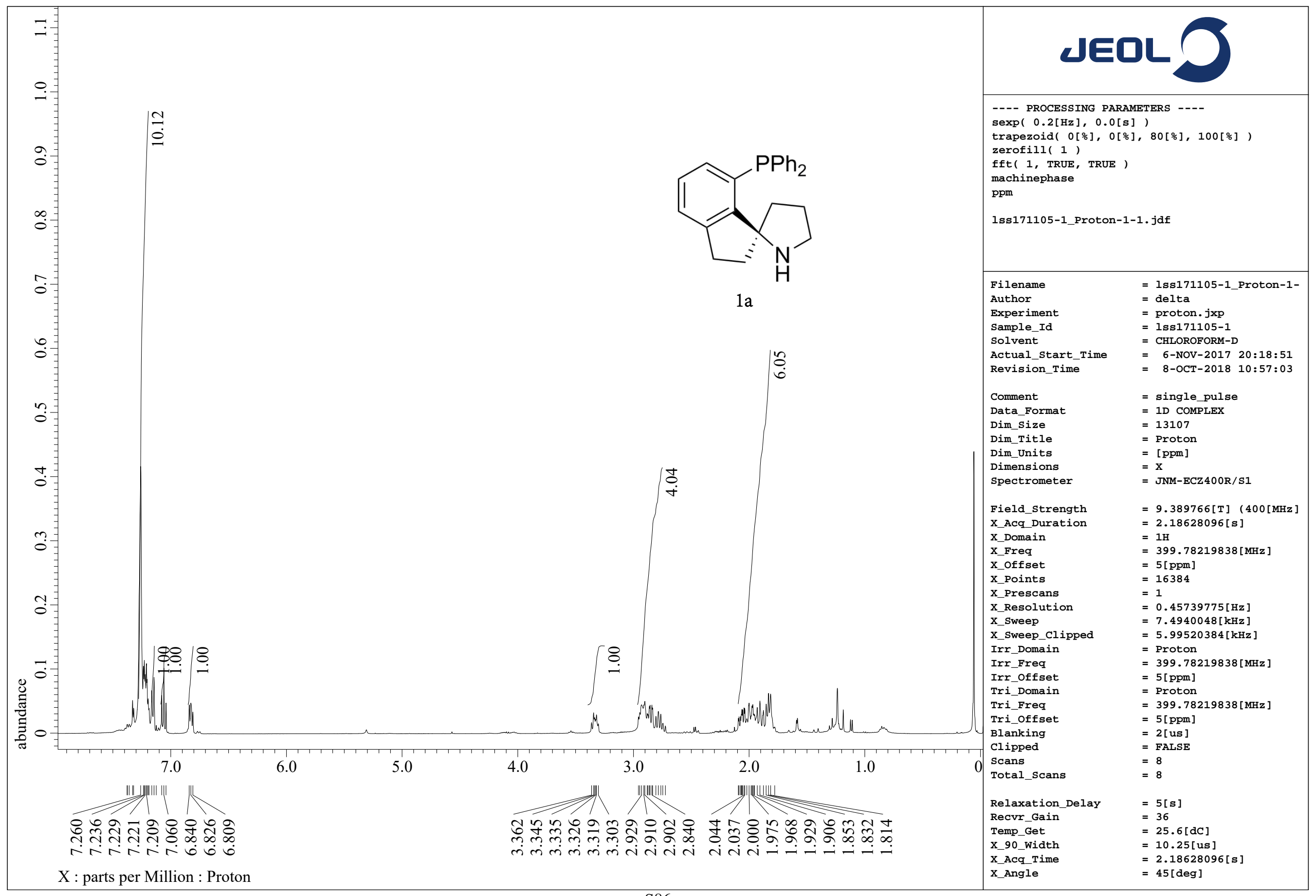




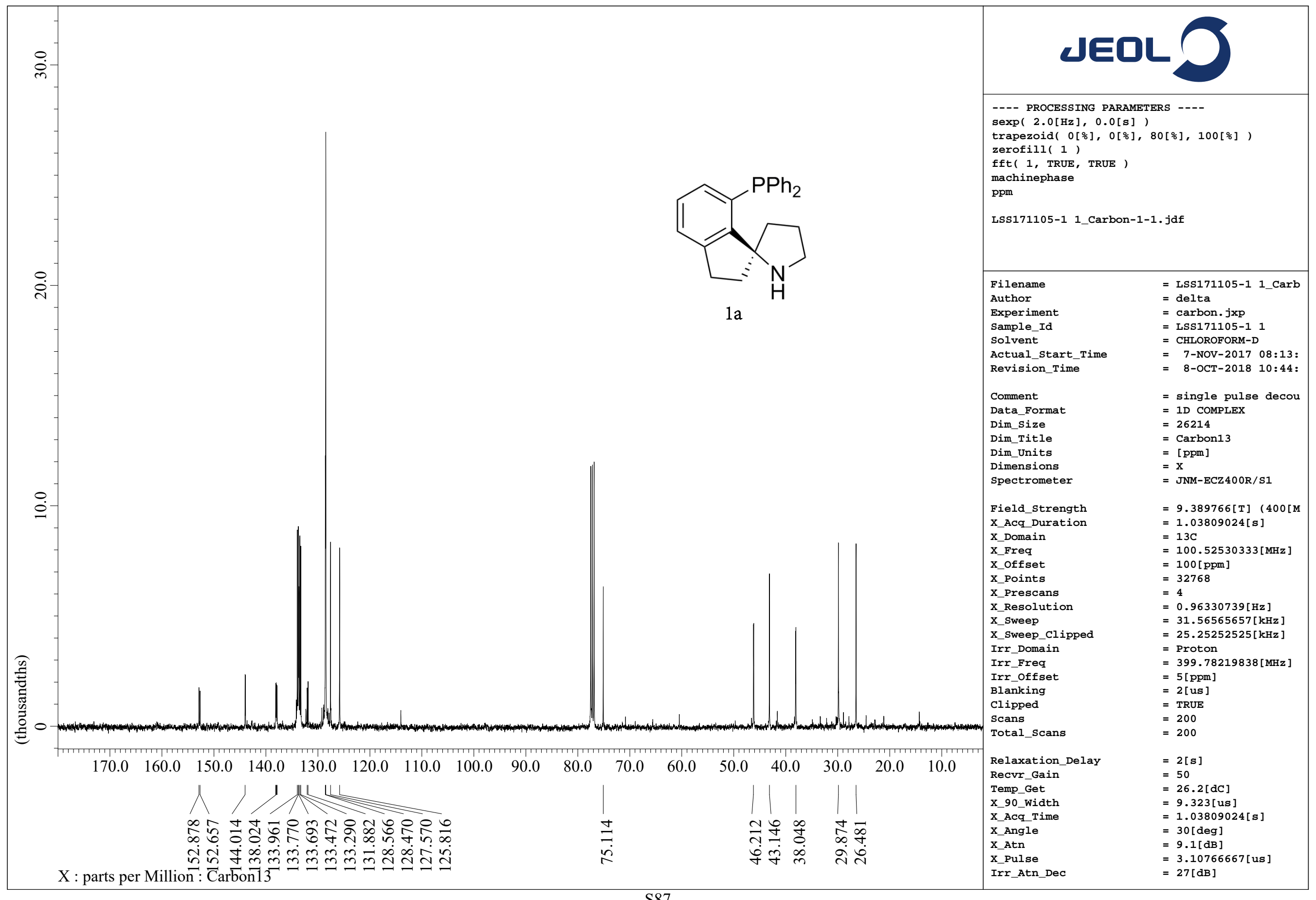




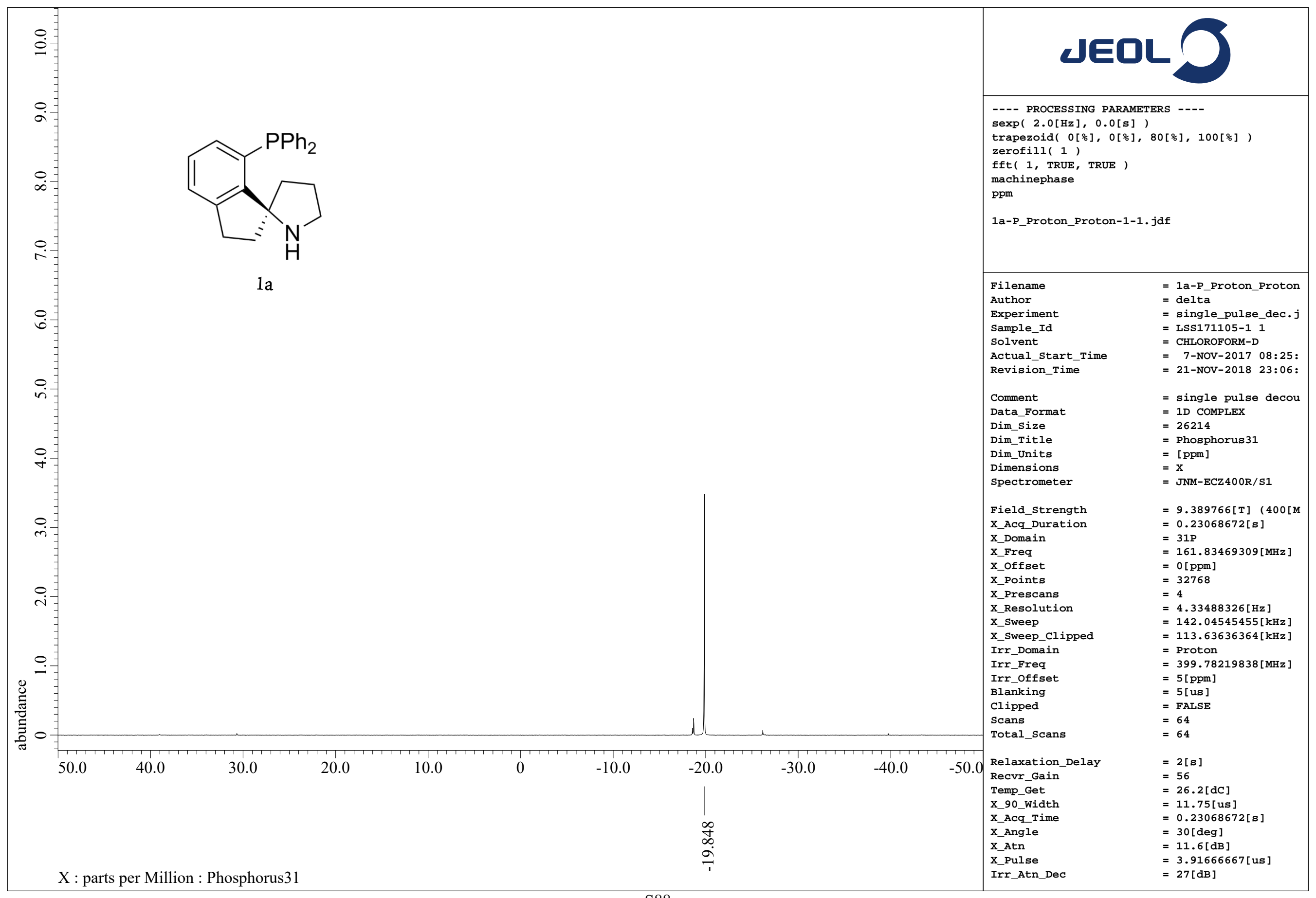




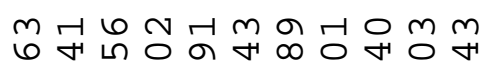
市 H ríñ $\longrightarrow$

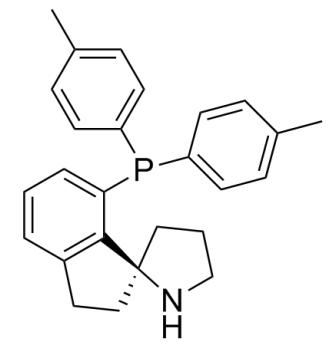

$1 \mathrm{~b}$
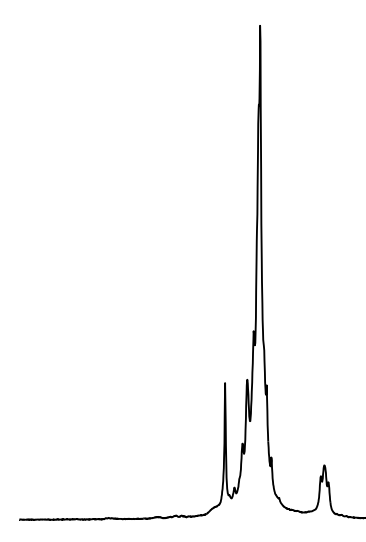

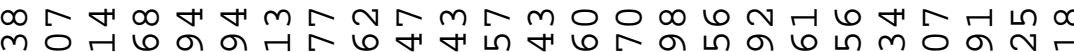
웅 ๑ m க்

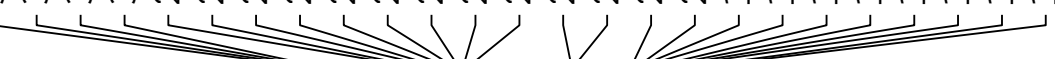

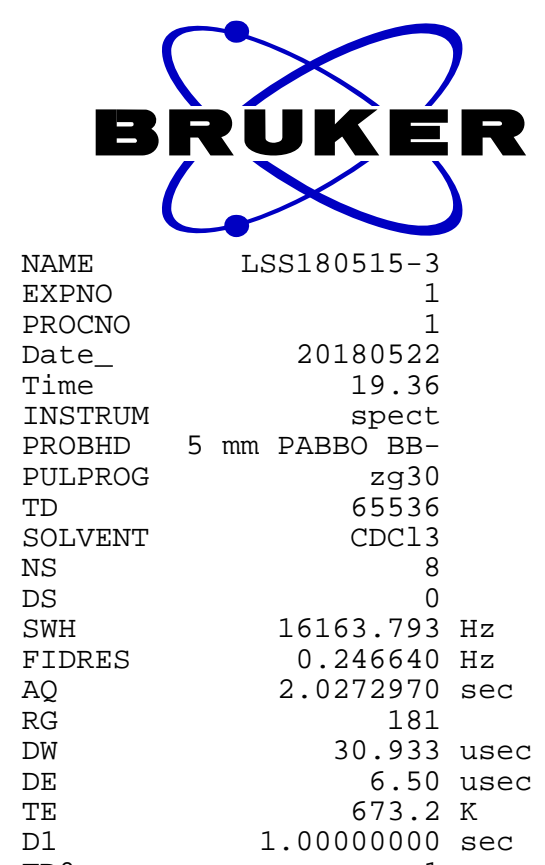

DO

$=======$ CHANNEL f1 ======== NUC1

P1

PL1

SF01

SF

WDW

SSB

GB

BRUMER

LS180515-3

0180522

$5 \mathrm{~mm}$ PABBO BB

$16163.793 \mathrm{~Hz}$ $\odot .24664 \odot \mathrm{Hz}$

181

$1.000000000 \mathrm{sec}$

15.50 usec

$-1.00 \mathrm{~dB}$

$13.18669796 \mathrm{~W}$

$400.1728012 \mathrm{MHz}$

32768

$400.1700337 \mathrm{MHz}$

$\mathrm{EM}$
0
$30 \mathrm{~Hz}$
0
0

$\begin{array}{llllllllllllllll}7.5 & 7.0 & 6.5 & 6.0 & 5.5 & 5.0 & 4.5 & 4.0 & 3.5 & 3.0 & 2.5 & 2.0 & 1.5 & 1.0 & \mathrm{ppm}\end{array}$

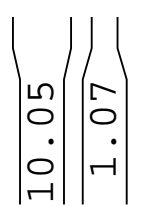




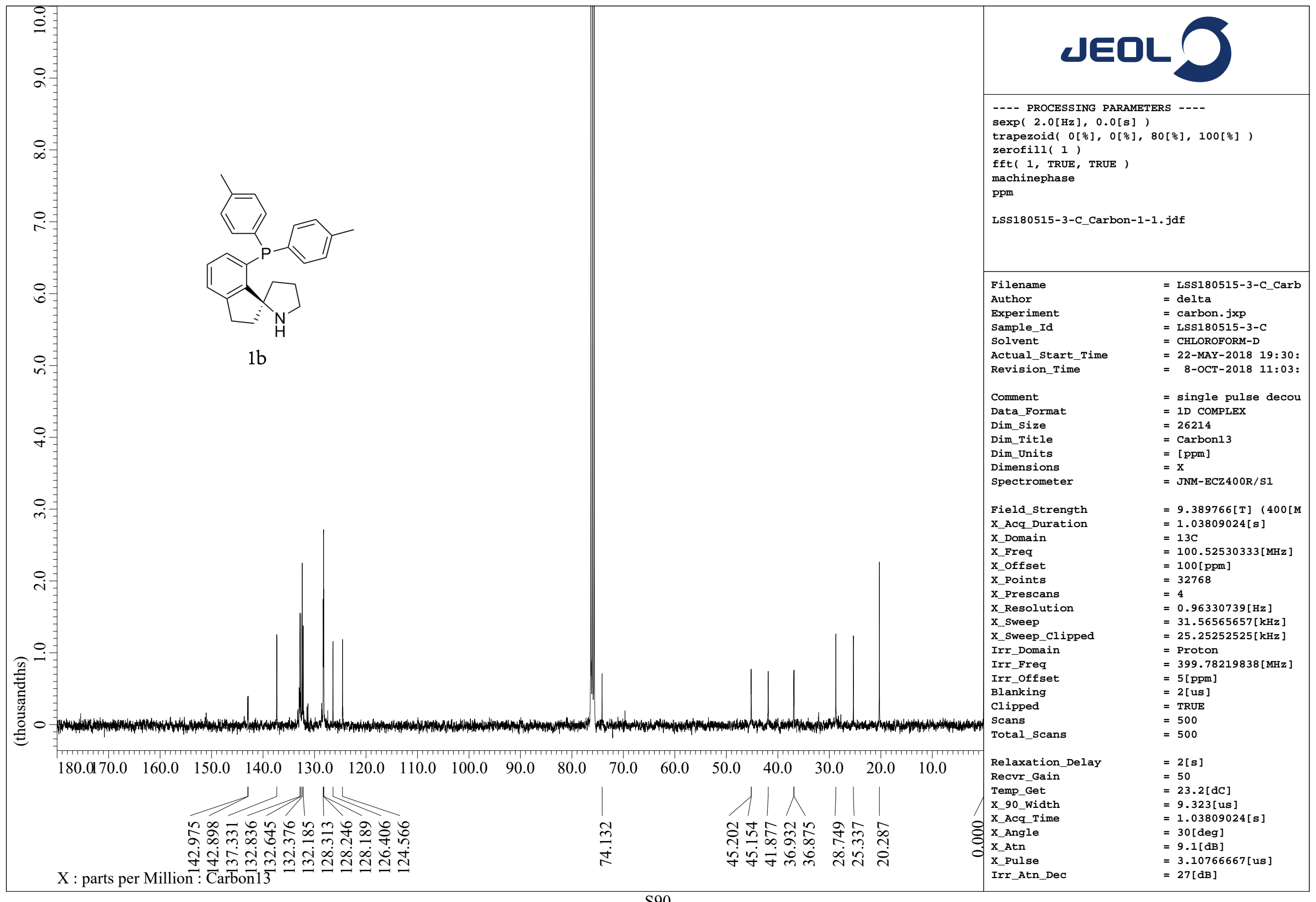




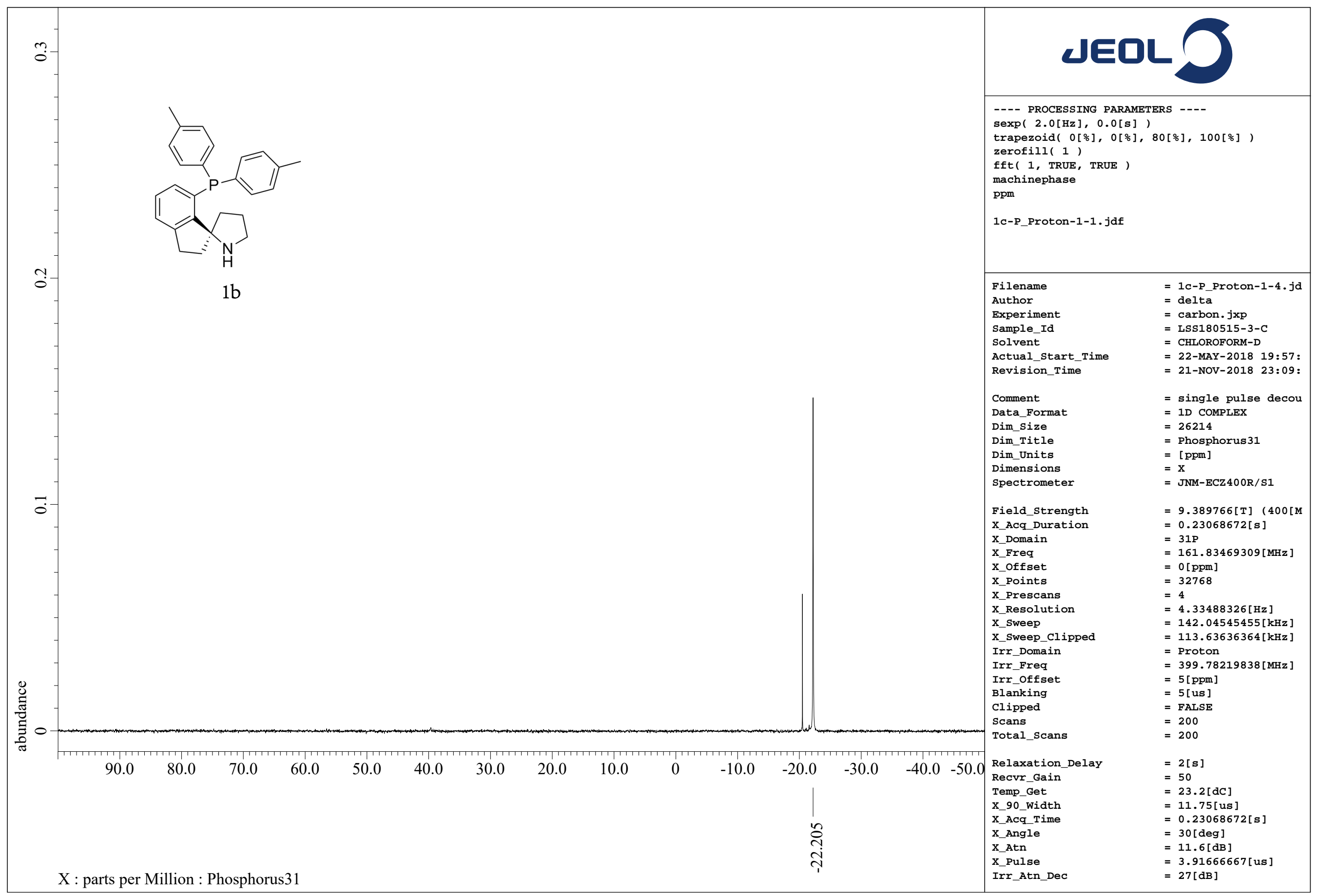


ט

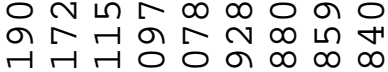
ה

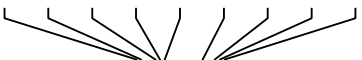

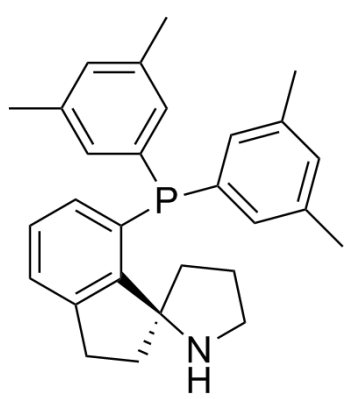

$1 c$ m

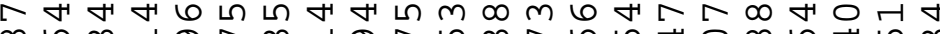
m m ब তু

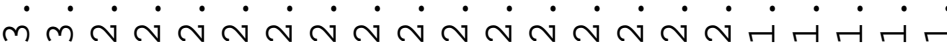

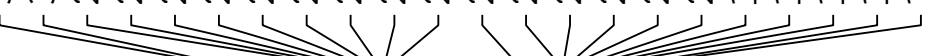

NAME

EXPNO

PROCN

Date

INSTRUM

PROBHD

PULPROG

TD

SOLVENT

DS

SWH

FIDRES

$A Q$

RG

DW

DE

TE

TDO

$=======$ CHANNEL

NUC1

PL1

PL1W

SF01

SF

WDW

SSB

LB

$\mathrm{GB}$
Bitiker

$C_{0}$

LSS180516-1

20180522 20.37

mm

zg30
65536

CDCl3

$16163.793 \mathrm{~Hz}$

$0.246640 \mathrm{~Hz}$

$2.0272970 \mathrm{sec}$

128

30.933 usec 6.50 usec $673.2 \mathrm{~K}$

$1.00000000 \mathrm{sec}$
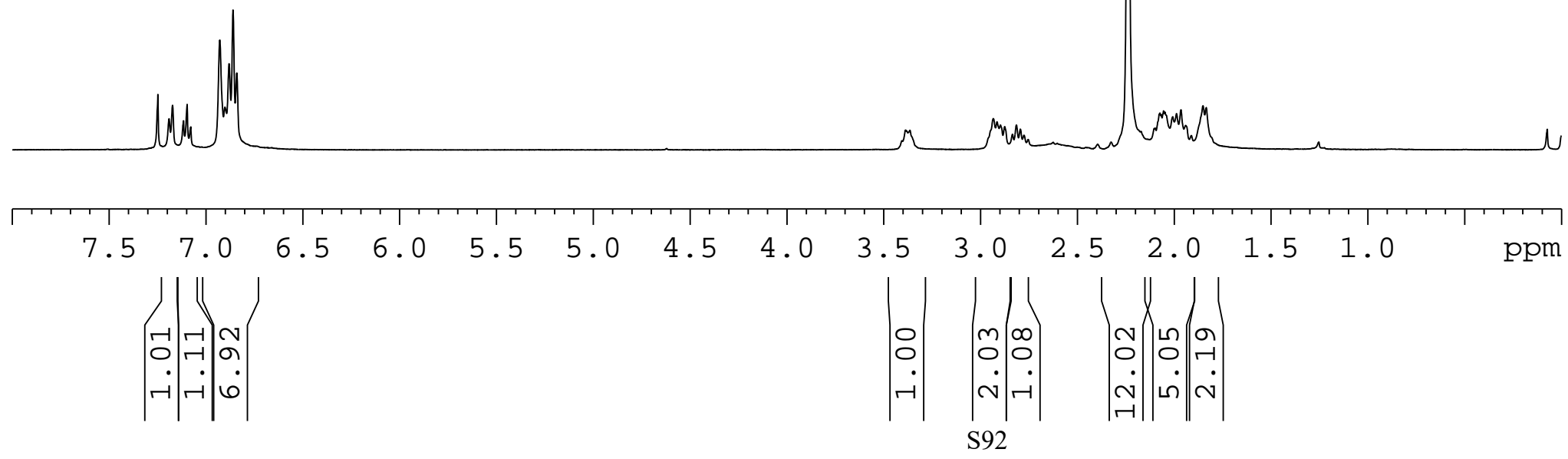

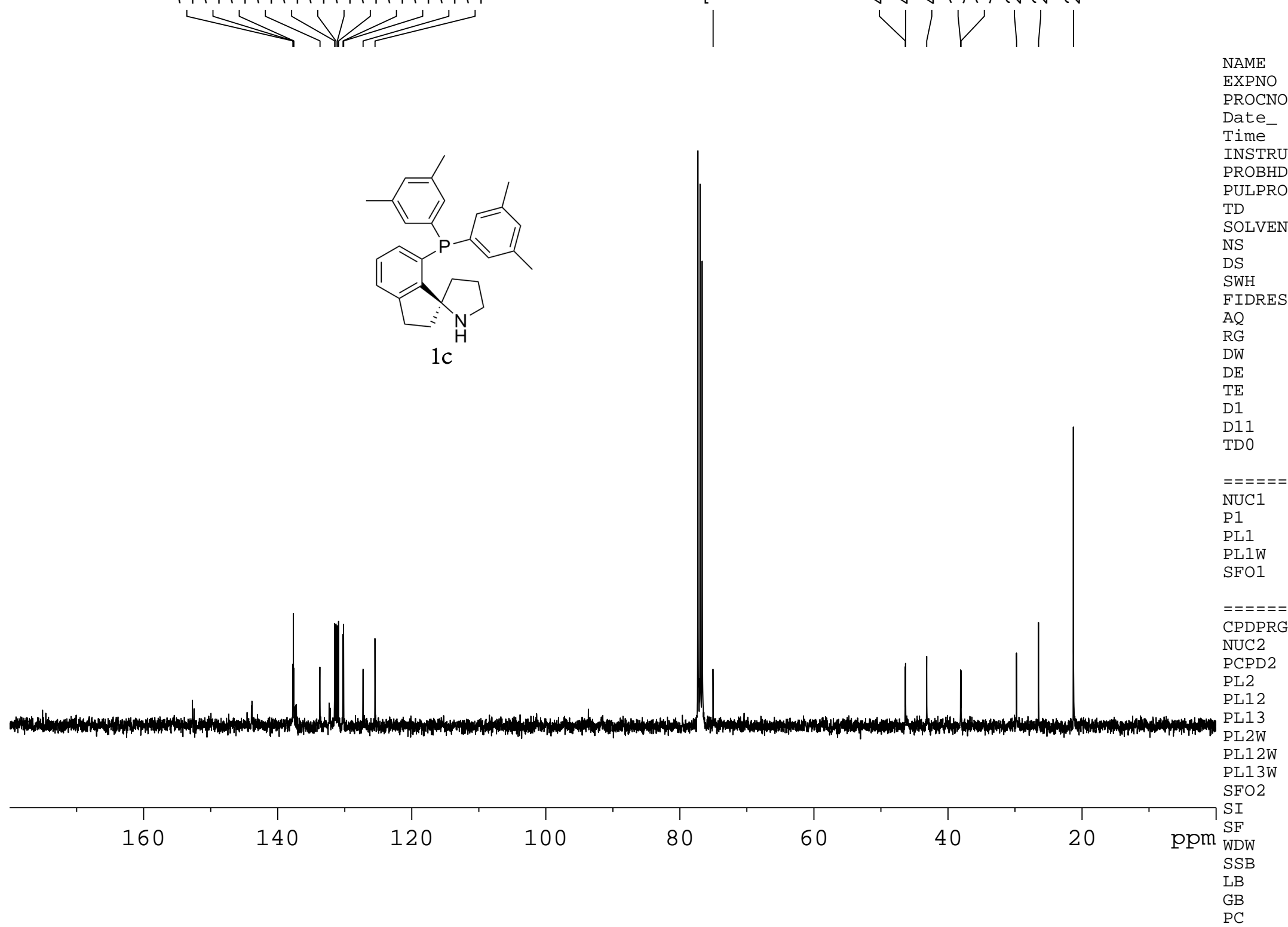

NAME

EXPNO

PROCNO

Date

ime

政

PULPROG

SWH

IDRES

RG

$\mathrm{DW}$

TE

D1

D11

$======$ CHANNEL

SF1W

$5 \mathrm{~mm}$ PABBO $\mathrm{spect}$

A

$\mathrm{zgpg} 30$
65536

CDCl3

200

$71428.570 \mathrm{~Hz}$

$1.089913 \mathrm{~Hz}$

$0.4588020 \mathrm{sec}$

203

7. 000 usec

6.50 usec

$673.2 \mathrm{~K}$

$2.00000000 \mathrm{Sec}$

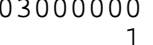

$====$

PRG

WDW

LB
$======-$

8.50 usec

$100.6228270 \mathrm{MHz}$

CHANNEL f2 ======== waltz16

80.00 usec $-1.00 \mathrm{~dB}$ $-1.00 \mathrm{~dB}$ $14.46 \mathrm{~dB}$ $13.18669796 \mathrm{~W}$ $0.49446553 \mathrm{~W}$ $0.37509048 \mathrm{~W}$ $400.1716007 \mathrm{MHz}$ 32768 $100.6228294 \mathrm{MHz}$ EM 1. $0 \odot \mathrm{Hz}$ $1.00 \mathrm{~Hz}$
$57.32743073 \mathrm{~W}$ 


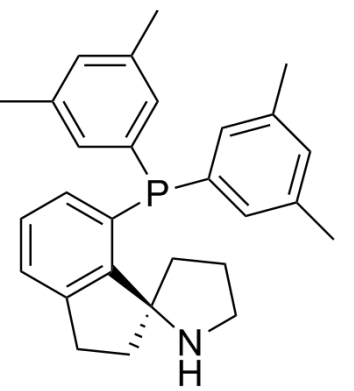

$1 c$


NAME

EXPNO

PROCNO

Date

Time

INSTRUM

PULPROG

PULP

TD

NS

DS

SWH
FIDRES

AQ

$\mathrm{RG}$

DW

$\mathrm{DE}$
$\mathrm{TE}$
$\mathrm{D} 1$

D1 1

TDO

BRUKER

$\mathrm{C}_{1}$

1ss180516-1-P

20180522

20.54

$5 \mathrm{~mm}$ spect

zgpg30

65536

$\mathrm{CDCl3}$

$98684.211 \mathrm{~Hz}$

$1.505802 \mathrm{~Hz}$

$0.3320991 \mathrm{sec}$

203

5.067 usec

6.50 usec

$673.2 \mathrm{~K}$

$2.00000000 \mathrm{sec}$

$0.03000000 \mathrm{~s}$

======== CHANNEL f1 ======= NUC1

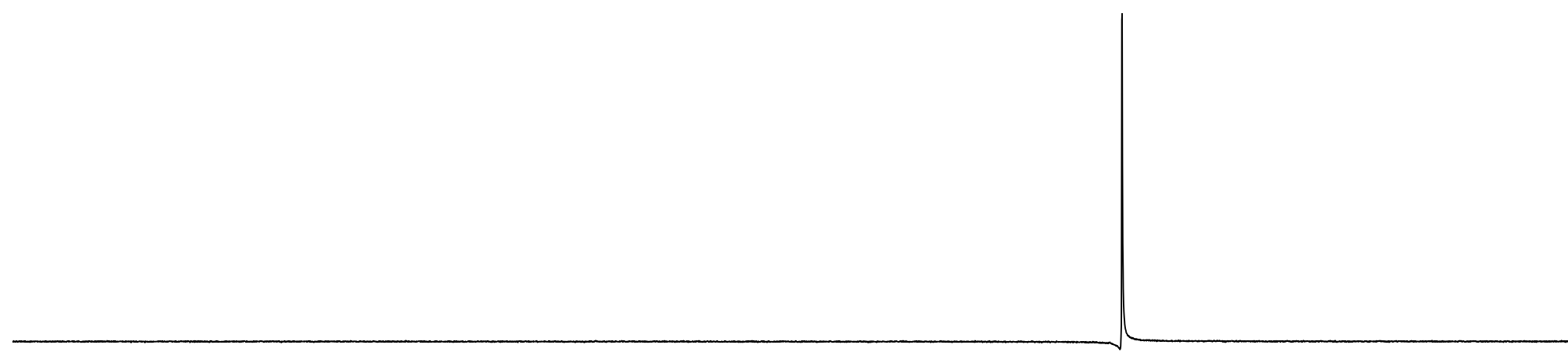

PL1W

8.80 usec

SF01

$30.16812897 \mathrm{~W}$

$161.9917850 \mathrm{MHz}$

$========$ CHANNEL f2 ========

CPDPRG2

NUC2

PCPD

PL2

PL13

PL2W

SF02

$1+1+1+1+1+190$

40

30

20

10

0

$-10$

$-20$

$-30$

$-40$

ppm

$f 2======$
waltz16
$1 \mathrm{H}$
$8 \odot .0 \odot \mathrm{usec}$
$-1.0 \odot \mathrm{dB}$
$13.26 \mathrm{~dB}$
$14.46 \mathrm{~dB}$
$13.18669796 \mathrm{~W}$
$0.49446553 \mathrm{~W}$
$0.37509048 \mathrm{~W}$
$40 \odot .1716007 \mathrm{MHz}$
$32768 \mathrm{MH}$
$161.991785 \odot \mathrm{MHz}$
$\mathrm{EM}$
$\odot$
$1.00 \mathrm{~Hz}$
$\ominus$
$1.4 \odot$




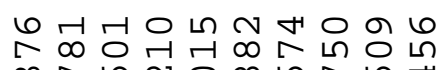
m sis

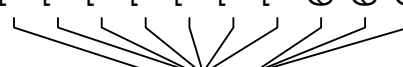

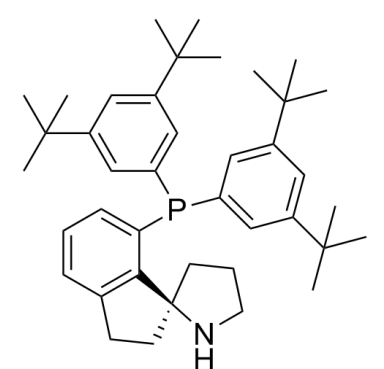

$1 d$

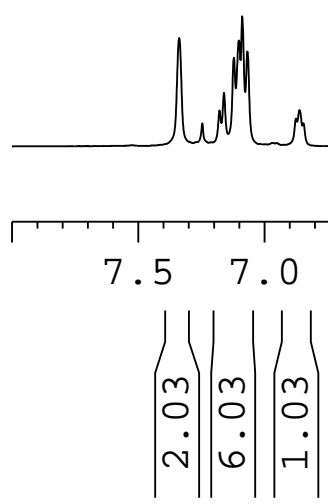

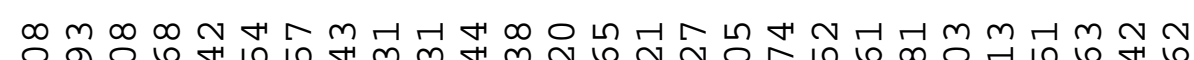

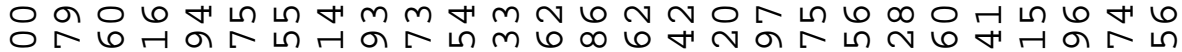

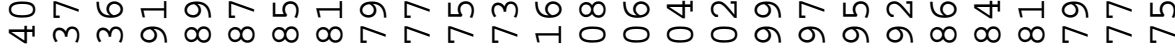

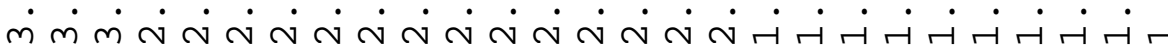

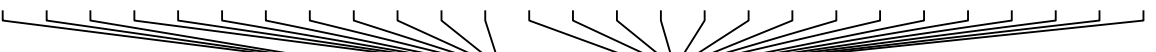

NAME

EXPNO

PROCNO

Date_

TIMSTRUM

PROBHD

PULPROG

TD

SOLVENT

NS

SWH

FIDRES

$\mathrm{AQ}$
$\mathrm{RG}$
$\mathrm{DW}$

DE

TE

TDO

$====$

NUC1

P1

PL1W

SFO1

SI

SF

WDW

LB

GB r ringer

LSS180907 -

20180907 11.25 $5 \mathrm{~mm}$ РABBO BB$\mathrm{zg} 30$ 65536 CDCl3 0

$16163.793 \mathrm{~Hz}$ $0.246640 \mathrm{~Hz}$ $2.0272970 \mathrm{sec}$ 50.8

30.933 usec $96.9 \mathrm{~K}$

$1.00000000 \mathrm{sec}$

$=$ CHANNEL $f 1$ 1 $==$ $-1.00 \mathrm{~dB}$ $13.18669796 \mathrm{~W}$ $400.1728012 \mathrm{MHz}$ 32768 $400.1700087 \mathrm{MHz}$ EM $0.30 \mathrm{~Hz}$ 1. 0 

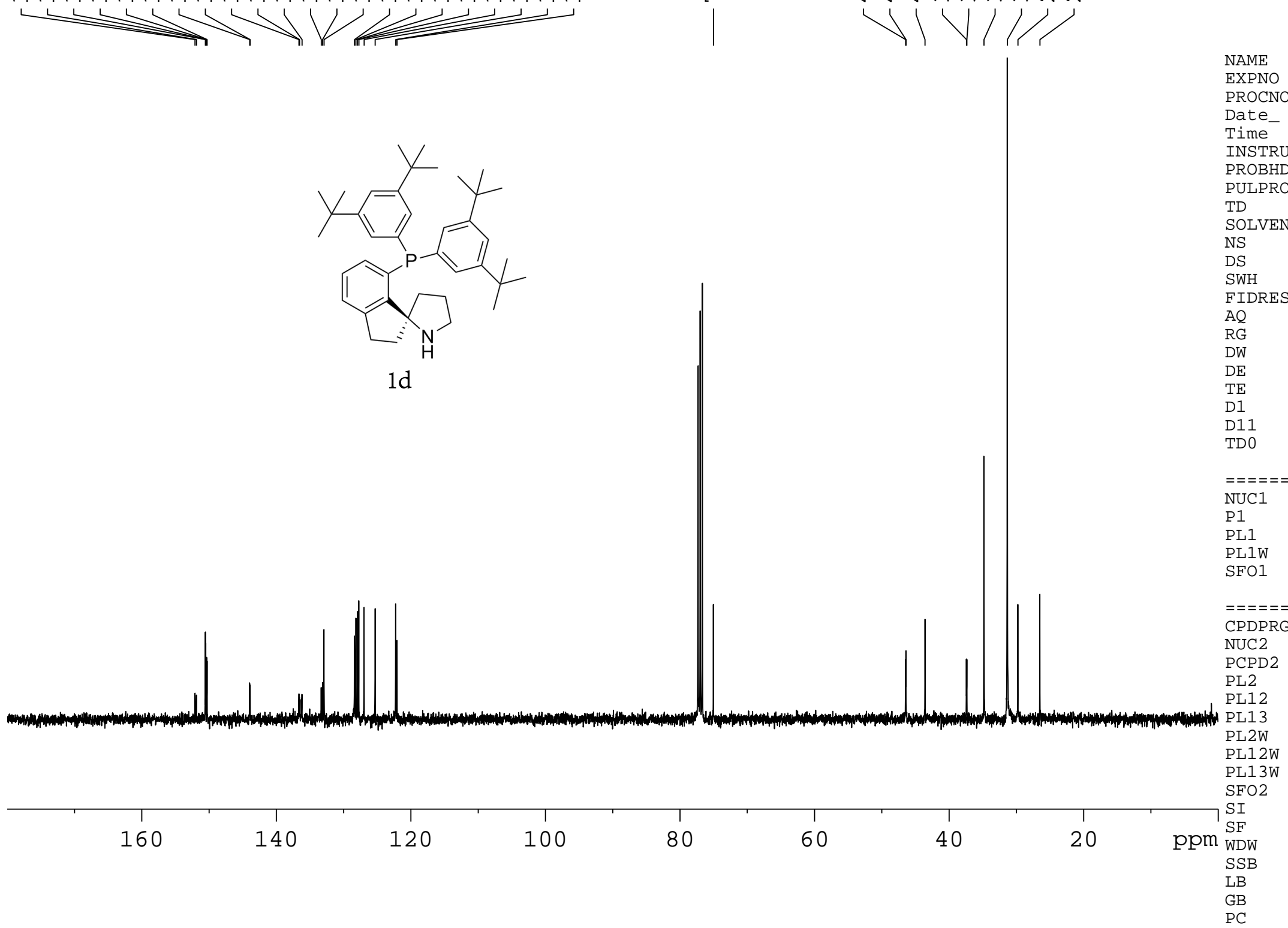

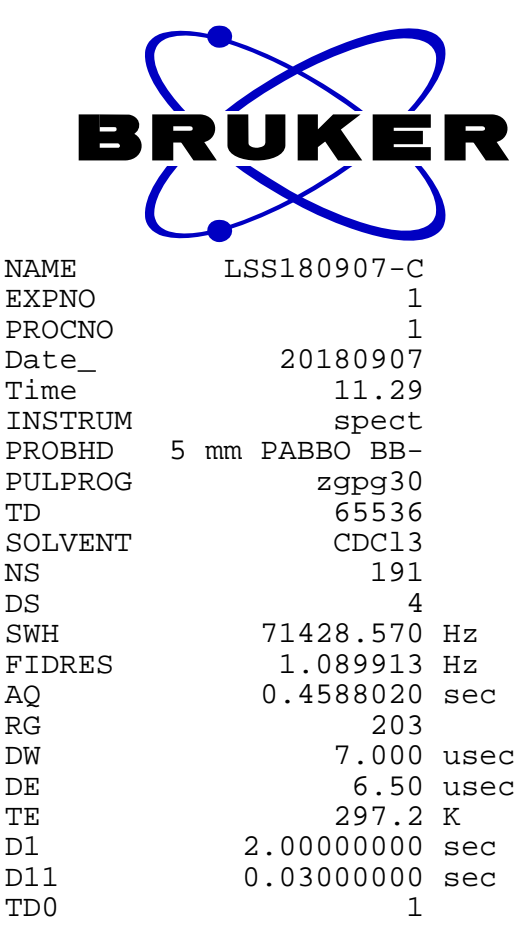

$=======$ CHANNEL NUC1

P1

PL1W

SF01

$======$

8.50 use

$2.00 \mathrm{~dB}$

$57.32743073 \mathrm{~W}$

$100.6228270 \mathrm{MHz}$

$========$ CHANNEL f2 ======== CPDPRG2

NUC2

PCPD

PL13

PL2W

PL13W

SF

SSB

$P C$

CHANNEL $f 2=======$
waltz16
$1 \mathrm{H}$
$80.00 \mathrm{usec}$
$-1.00 \mathrm{~dB}$
$13.26 \mathrm{~dB}$
$14.46 \mathrm{~dB}$
$13.18669796 \mathrm{~W}$
$0.49446553 \mathrm{~W}$
$0.37509048 \mathrm{~W}$
$400.1716007 \mathrm{MHz}$
$32768 \mathrm{MH}$
$100.6228294 \mathrm{MHz}$
$\mathrm{EM}$
$\odot$
$1.00 \mathrm{~Hz}$
$\ominus$
1.40




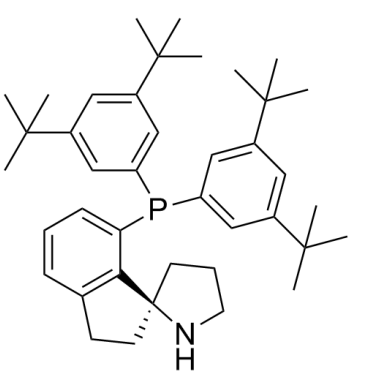

$1 d$

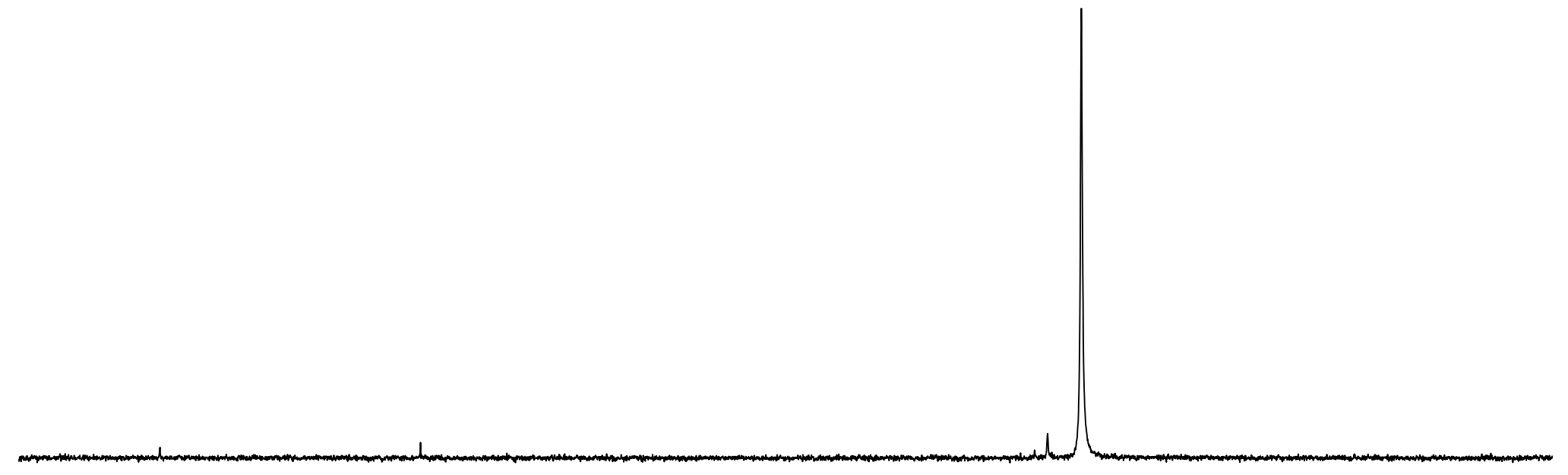

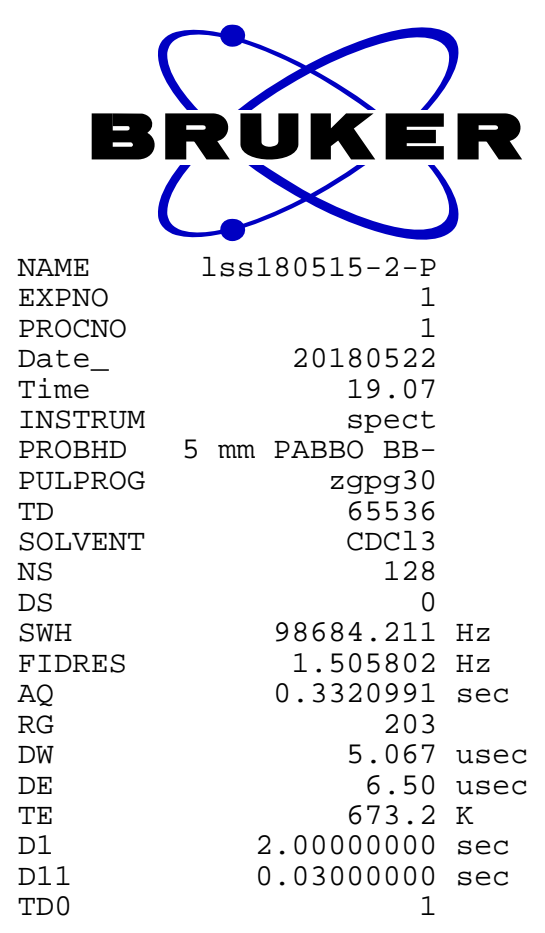

$=======$ CHANNEL NUC1

PL1W 8.80 use $1.00 \mathrm{~dB}$

$========$ CHANNEL f2 ======== CPDPRG2

NUC2

PCPD2

PL2

PL12

PL2W

PL12W

PL13W

SI

WDW

SSB

GB

$\mathrm{GB}$

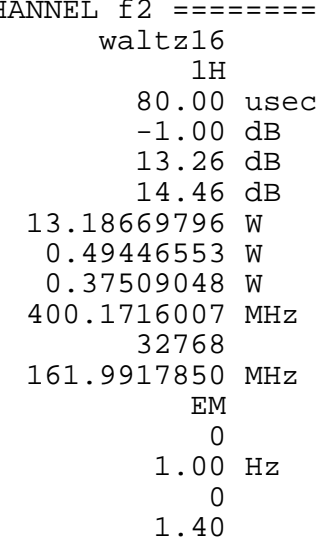




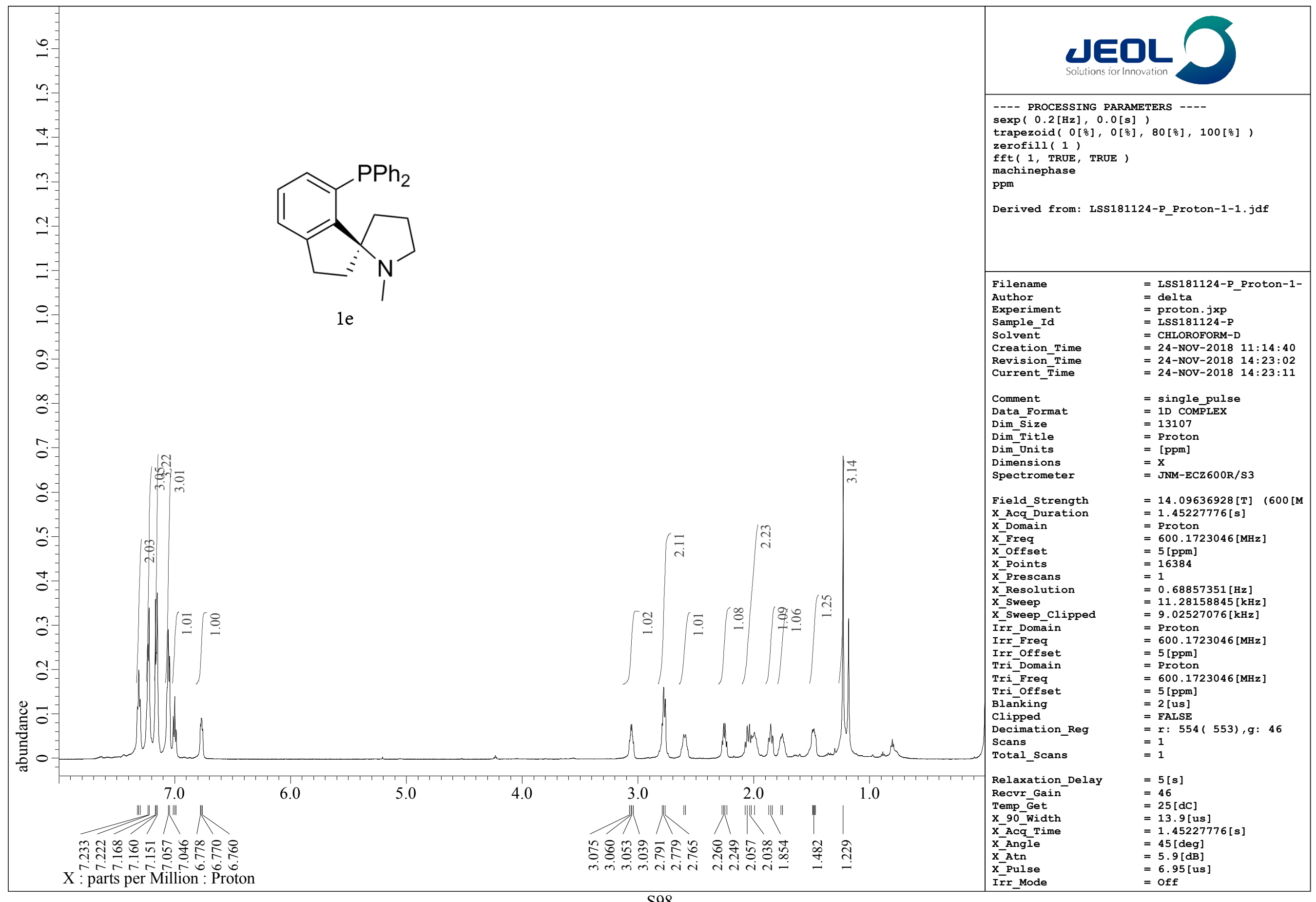



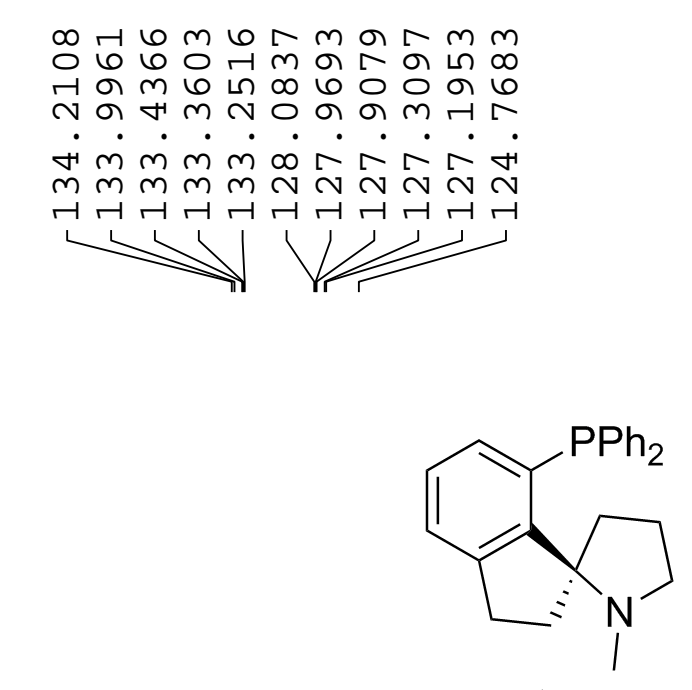

1e
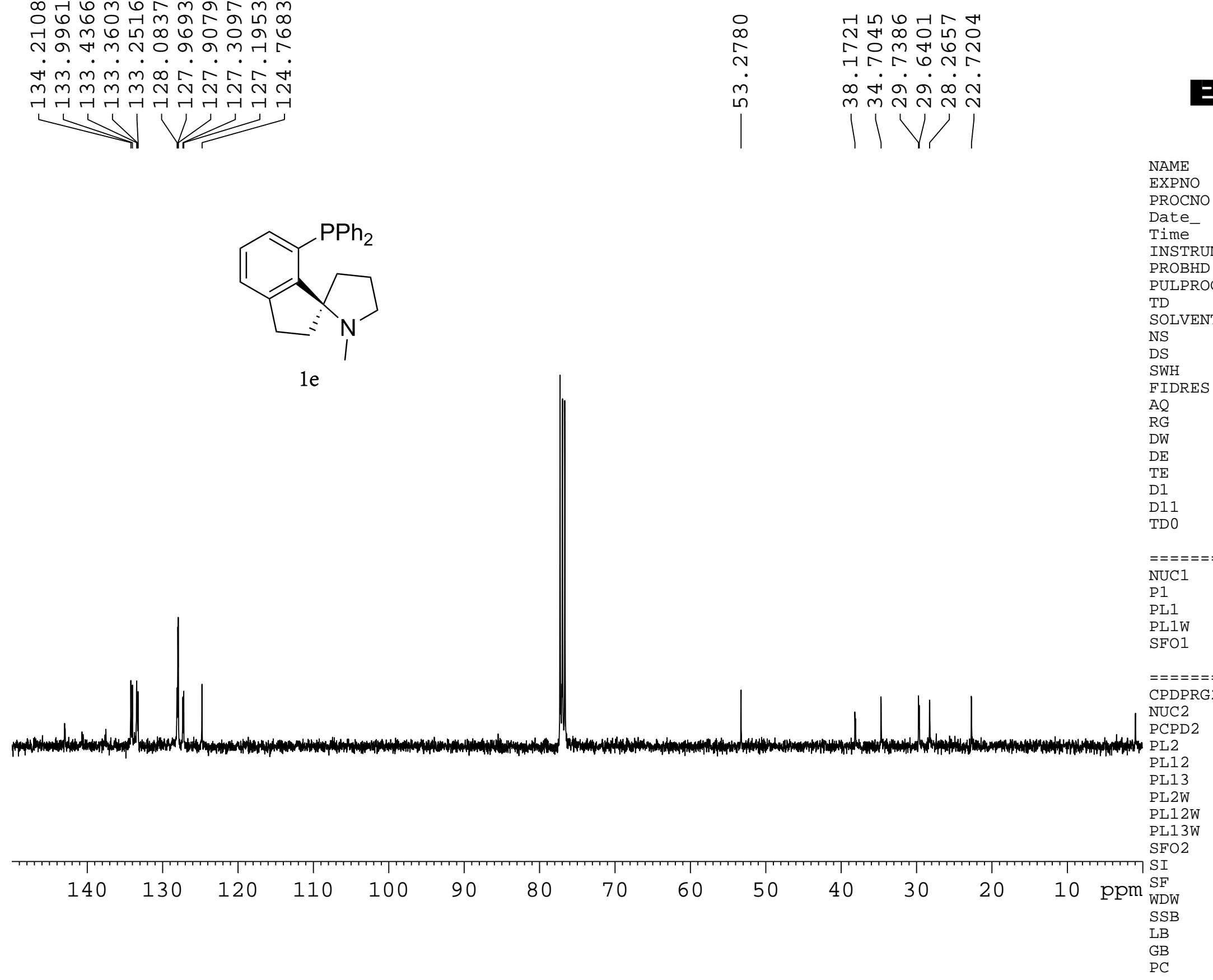

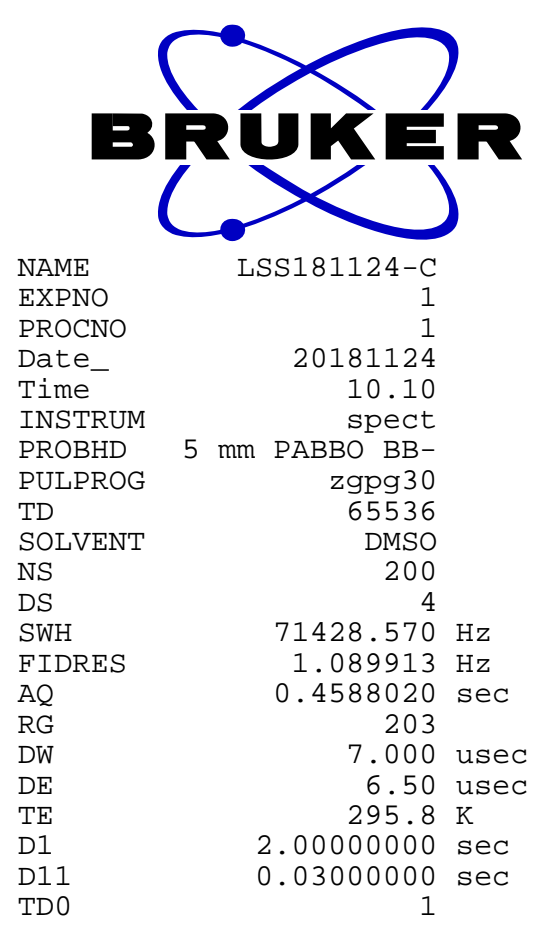

= $====$ CHANNEL N1 1 $100.6228270 \mathrm{MHz}$

(HANNEL f2 ======== PDPRG

PL

SF

LB

$P C$

$f 2======$
waltz16
$1 \mathrm{H}$
$80.0 \odot \mathrm{usec}$
$-1.00 \mathrm{~dB}$
$13.26 \mathrm{~dB}$
$14.46 \mathrm{~dB}$
$13.18669796 \mathrm{~W}$
$0.49446553 \mathrm{~W}$
$0.37509048 \mathrm{~W}$
$40 \odot .1716007 \mathrm{MHz}$
$32768 \mathrm{MH}$
$100.6223545 \mathrm{MHz}$
$\mathrm{EM}$
$\odot$
$1.0 \odot \mathrm{Hz}$
$\ominus$
1.40




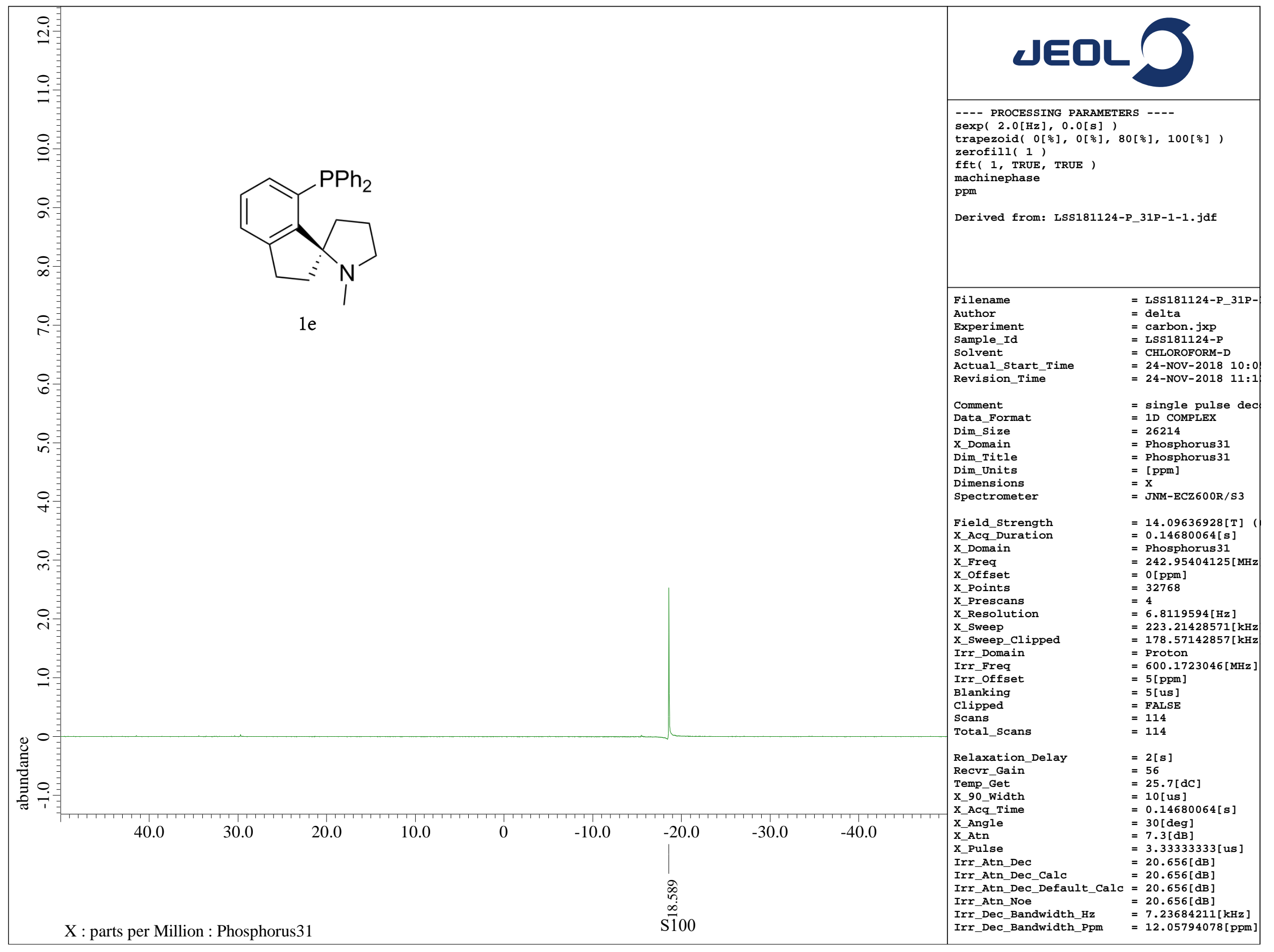




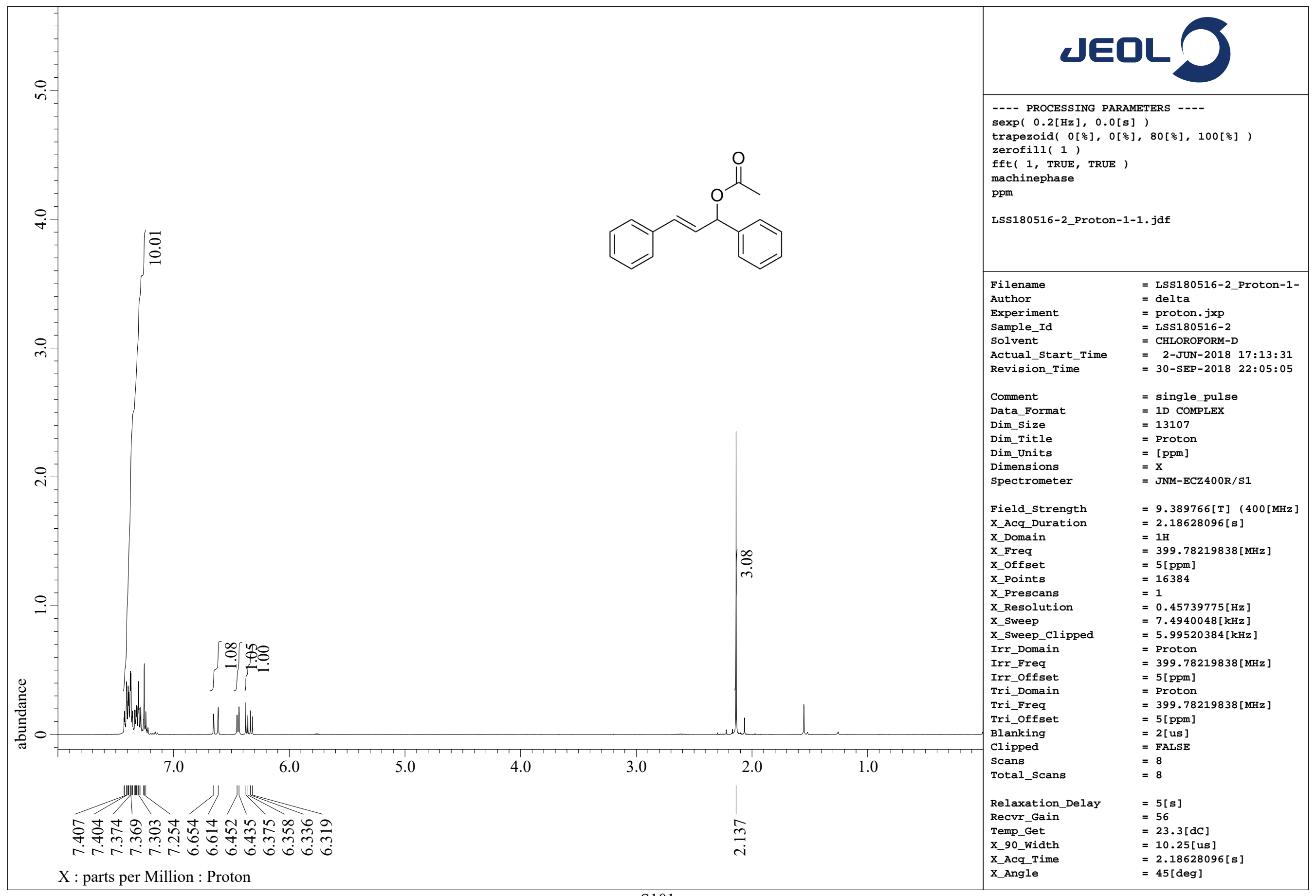



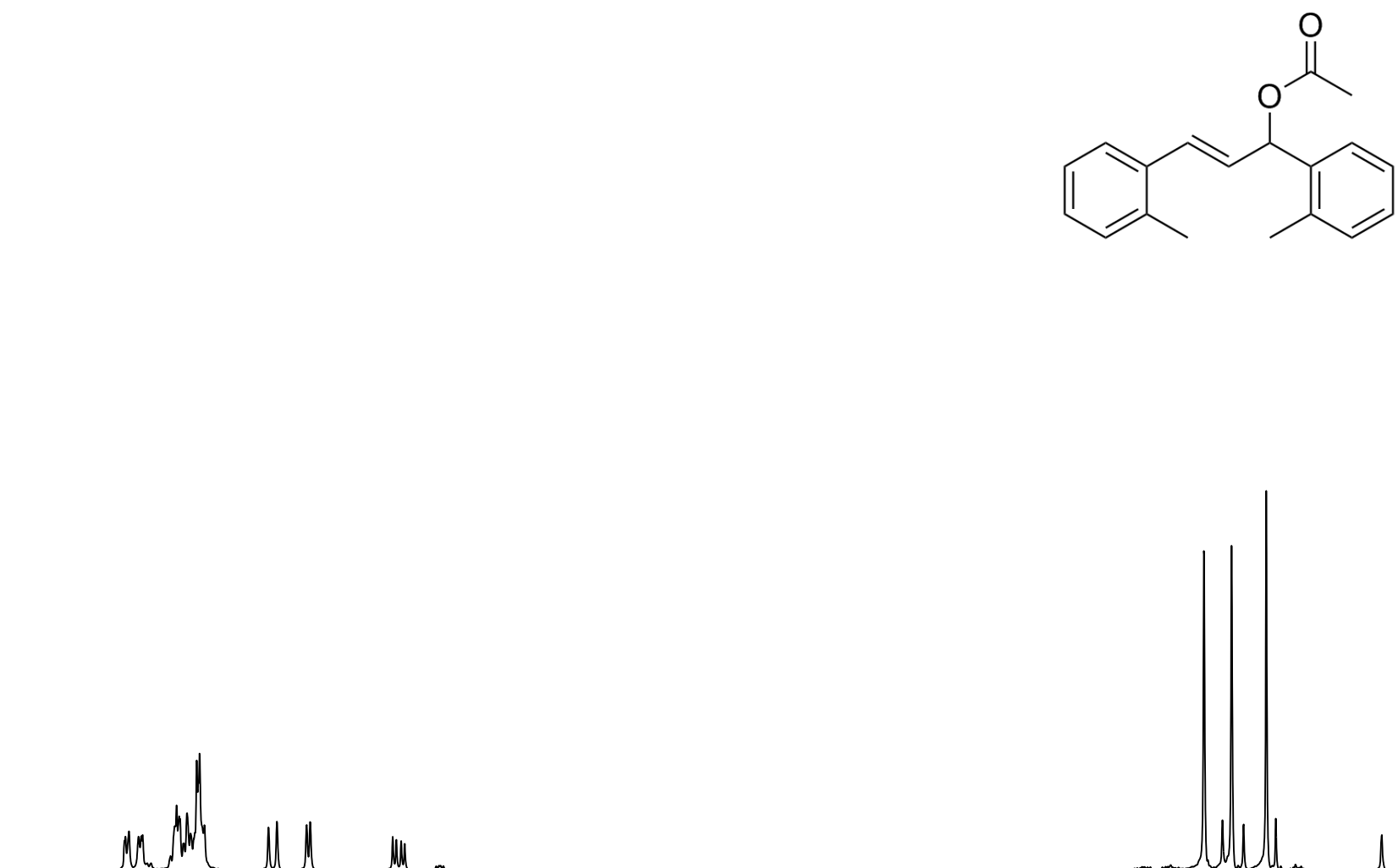

NAME

EXPNO

PROCNO

Date

PROBHD

PULPROG

TD

SOLVENT

NS
DS
SWH

SWH
FIDRES

$A Q$

RG

DW

DE

D1

TDQ

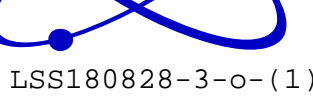

LSS180828-3-0-(1)

20180901 8.57 $5 \mathrm{~mm}$ РABBO BB-

$\mathrm{zg} 30$

65536

$\mathrm{CDCl3}$

16163. $\stackrel{0}{\circ} \mathrm{Hz}$

$0.246640 \mathrm{~Hz}$

$2.0272970 \mathrm{sec}$

71.8

30.933 usec
6.50 usec $97.4 \mathrm{~K}$

$1.00000000 \mathrm{sec}$

NUC1

P1

PL1

SF01

SF

WDW

SSB

(

$P C$

CHANNEL

$======$

15.50 usec

$-1.00 \mathrm{~dB}$

$13.18669796 \mathrm{~W}$

$400.1728012 \mathrm{MHz}$

32768

400.1700147 MHz

EM

$\odot .30 \mathrm{~Hz}$

1.00

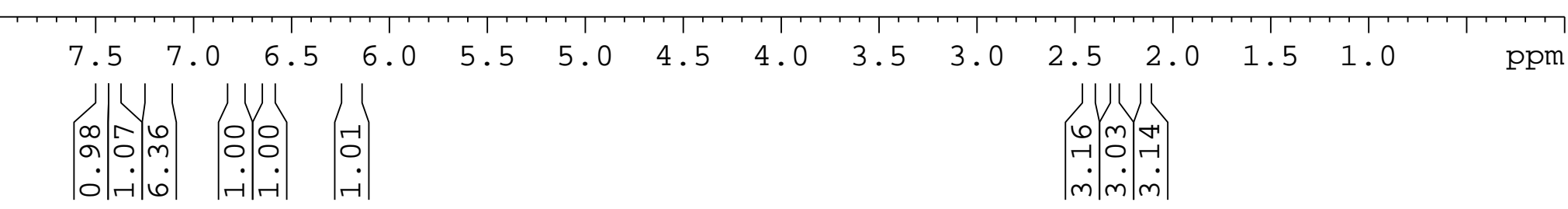




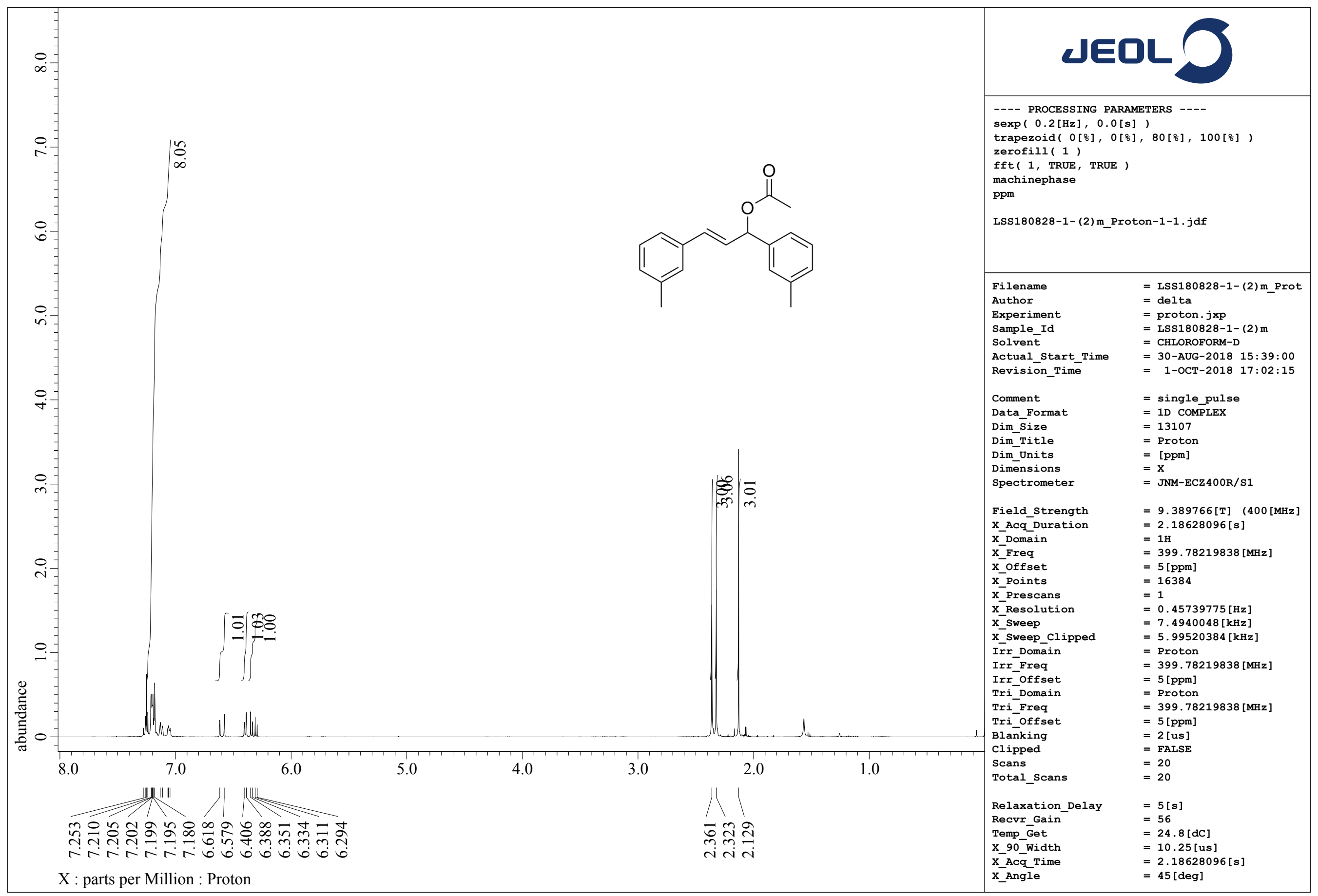




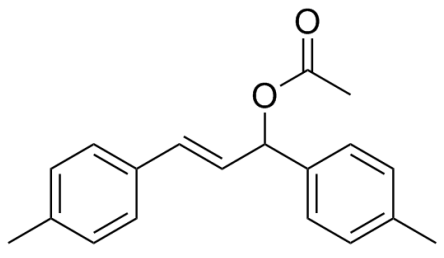

NAME

EXPNO

PROCNO

Date

Time-

INSTRUM

PROBHD

PULPROG

TD

SOLVENT

NS
DS
SWH

SWH

AQ

RG

DW

TE

D1

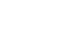
NUC1 P1

PL1

SF01

SI

SF

mu MUn

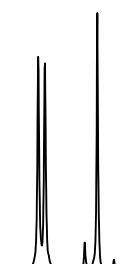

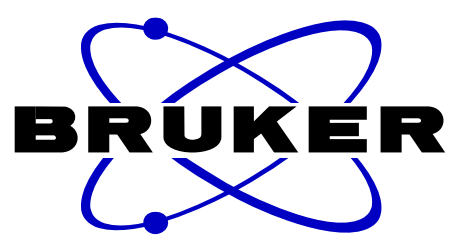

LSS180820-1-

20180830 19.36 $5 \mathrm{~mm}$ РABBO BBzg30 65536 $\mathrm{CDCl3}$ 10
0

$16163.793 \mathrm{~Hz}$ $0.246640 \mathrm{~Hz}$ $2.0272970 \mathrm{sec}$ 80.6

30.933 usec (9.3 K

$1.00000000 \mathrm{sec}$

$=$ CHANNEL $\mathrm{f}$

$======$
$1 \mathrm{H}$ 15.50 usec $13.18669796 \mathrm{~W}$ $400.1728012 \mathrm{MHz}$ 32768 $400.1700117 \mathrm{MHz}$ EM $0.30 \mathrm{~Hz}$ 0
1.00

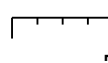

$7.5 \quad 7.0$

6.56 .0

5.5

5.04 .5

4.0

3.5

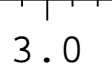

2.5

2.0

ppm

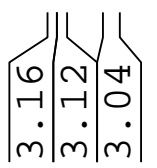

궁

लालm 
๑๑ீ๐ 강 m $m \stackrel{\infty}{\sim} \stackrel{\infty}{\sim}$ ก r M1
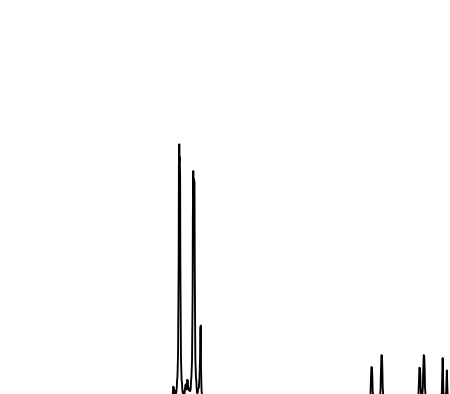
NIIIII

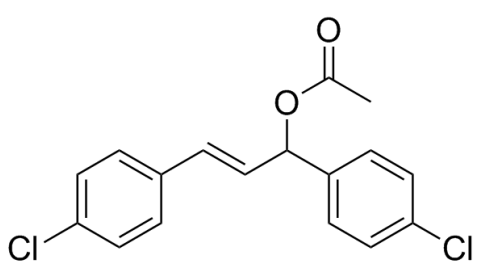

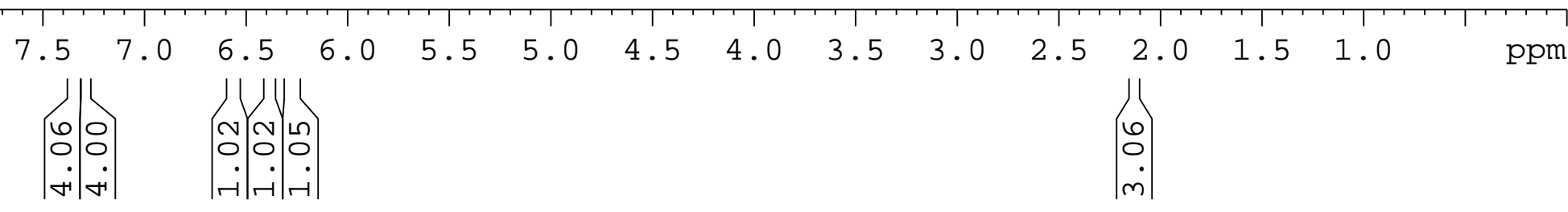

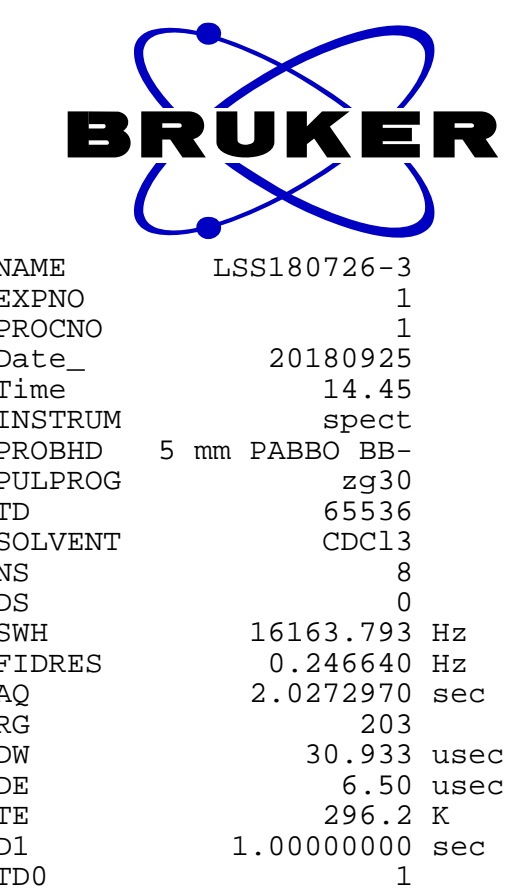

$=======$ CHANNEL NUC1 15.50 usec $-1.00 \mathrm{~dB}$
$13.18669796 \mathrm{~W}$ $400.1728012 \mathrm{MHz}$ 32768 $400.1700041 \mathrm{MHz}$ $\mathrm{EM}$
$\ominus$
$0.30 \mathrm{~Hz}$ 0.30
0
1.00 


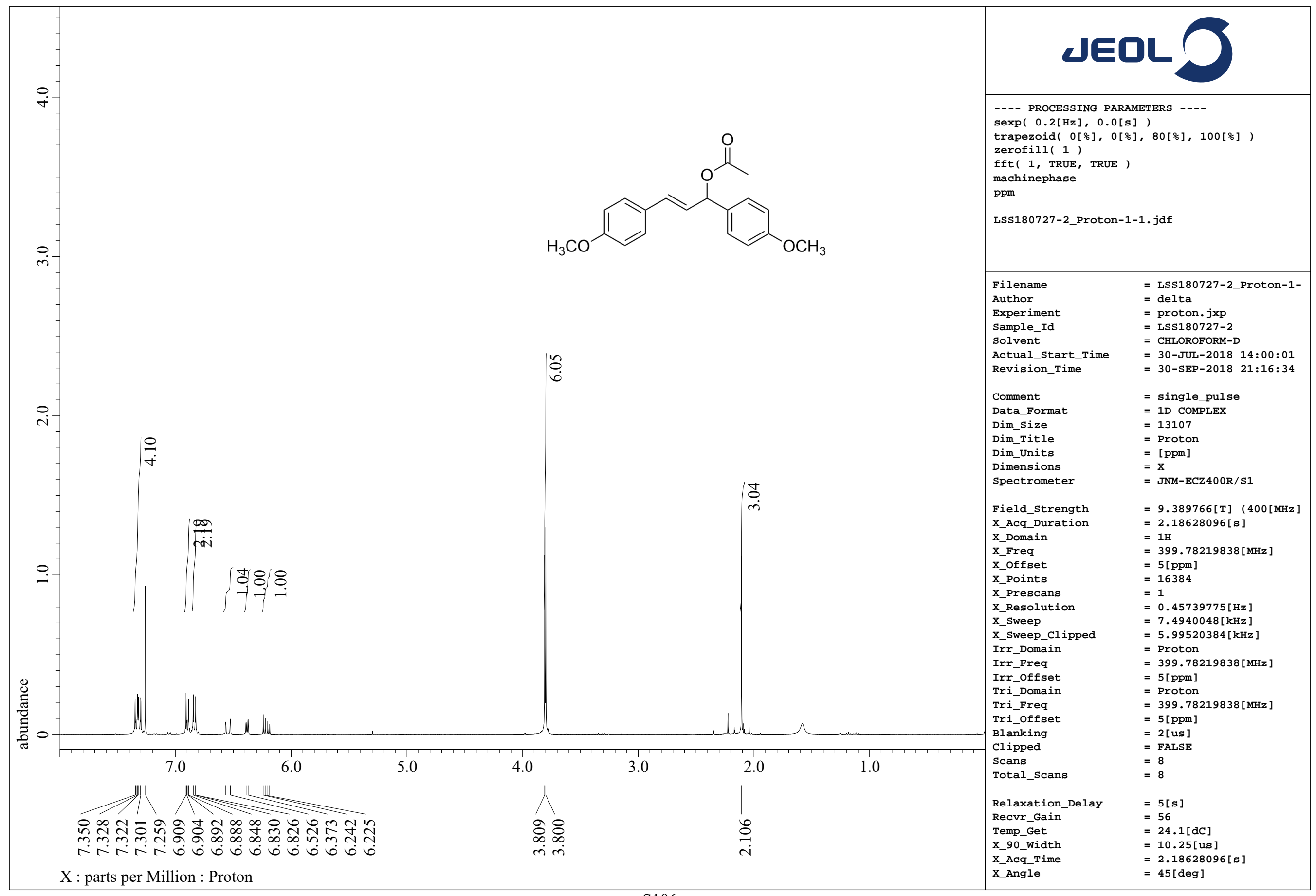




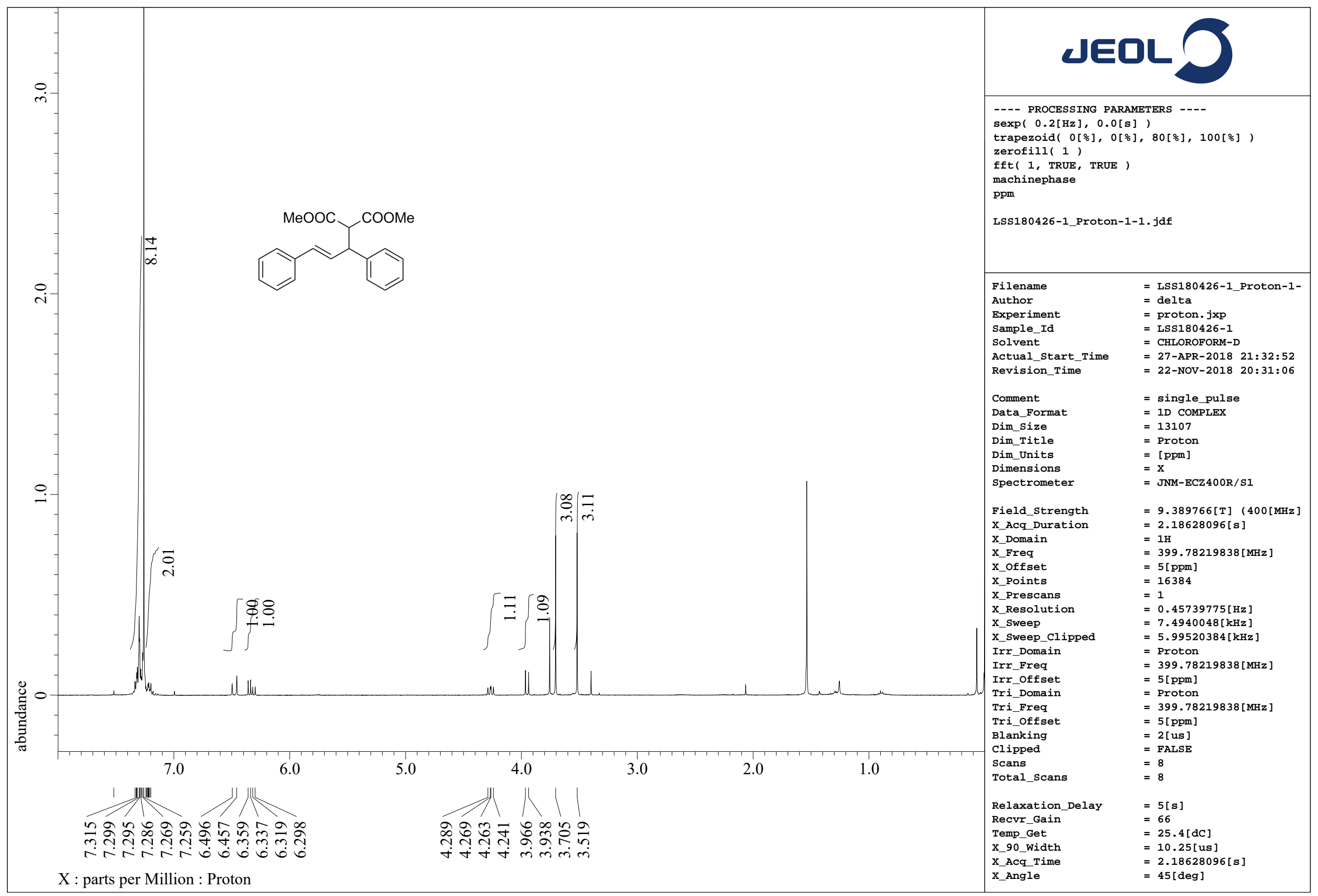


m 只

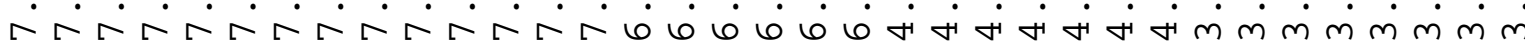

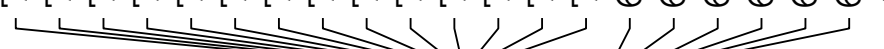
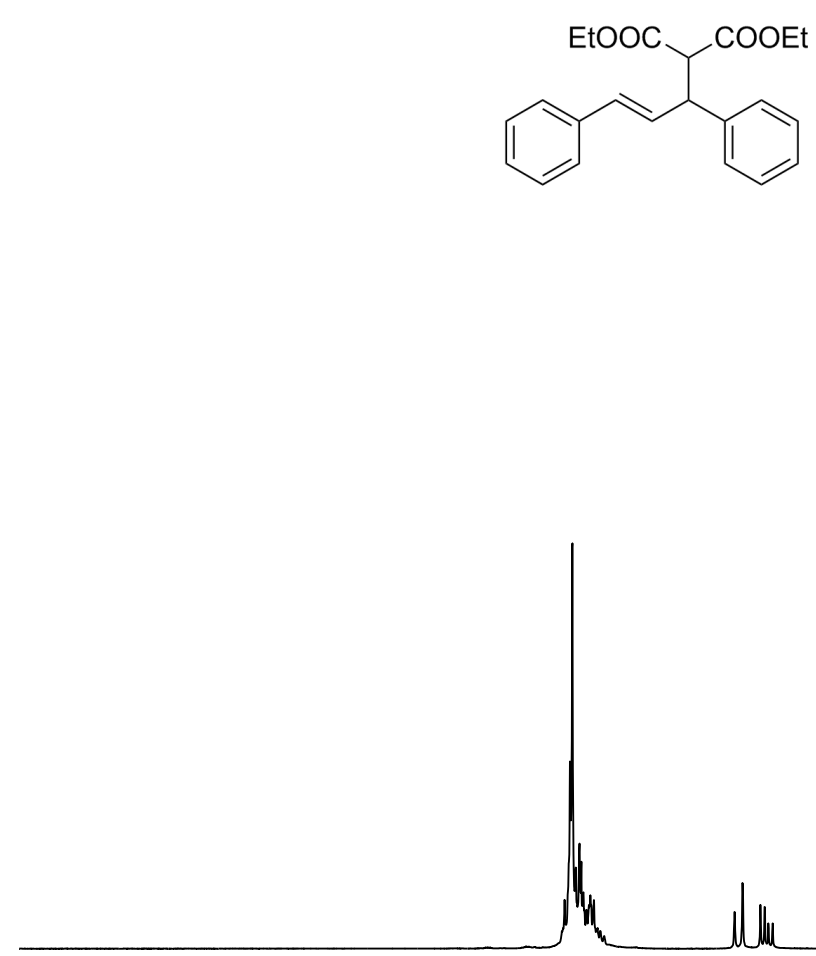

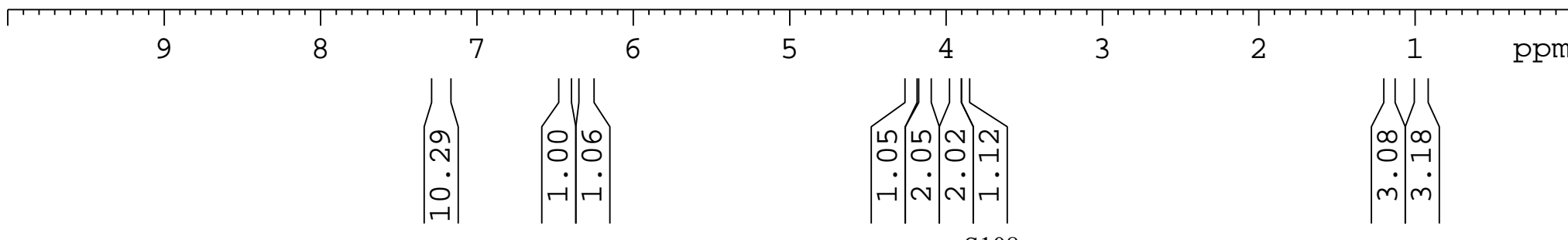

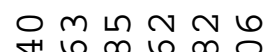
$\infty$ 0 ษ 4 31
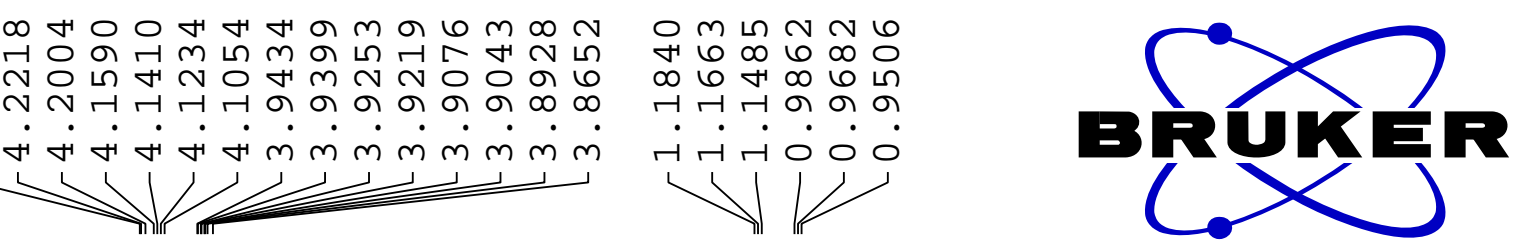

NAME

EXPNO

PROCNO

Date

INIme

PROBHD

PROBHD
PULPROG

PULP

SOLVENT

NS

DS

SWH
FIDRES

AQ

RG

DW

DE

TE

TD

SS180419-2 THF

20180420

15.43

$5 \mathrm{~mm}$ spect

Bg-
zg30

$\mathrm{Zg} 30$
65536

CDCl3

$\Theta$
$16163.793 \mathrm{~Hz}$

$0.246640 \mathrm{~Hz}$

$2.0272970 \mathrm{sec}$

90.5

30.933 usec

6.50 usec

$673.2 \mathrm{~K}$

$1.00000000 \mathrm{sec}$

NUC1

PL

PL1W

SF01

SF

WDW

SSB

LB

PC

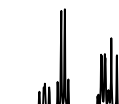

========

15.50 usec

$13.18669796 \mathrm{~W}$

$400.1728012 \mathrm{MHz}$

32768

$400.1700207 \mathrm{MHz}$

$$
\begin{gathered}
\text { EM } \\
\Theta \\
\odot .30 \mathrm{~Hz}
\end{gathered}
$$$$
1.0 \odot
$$ 


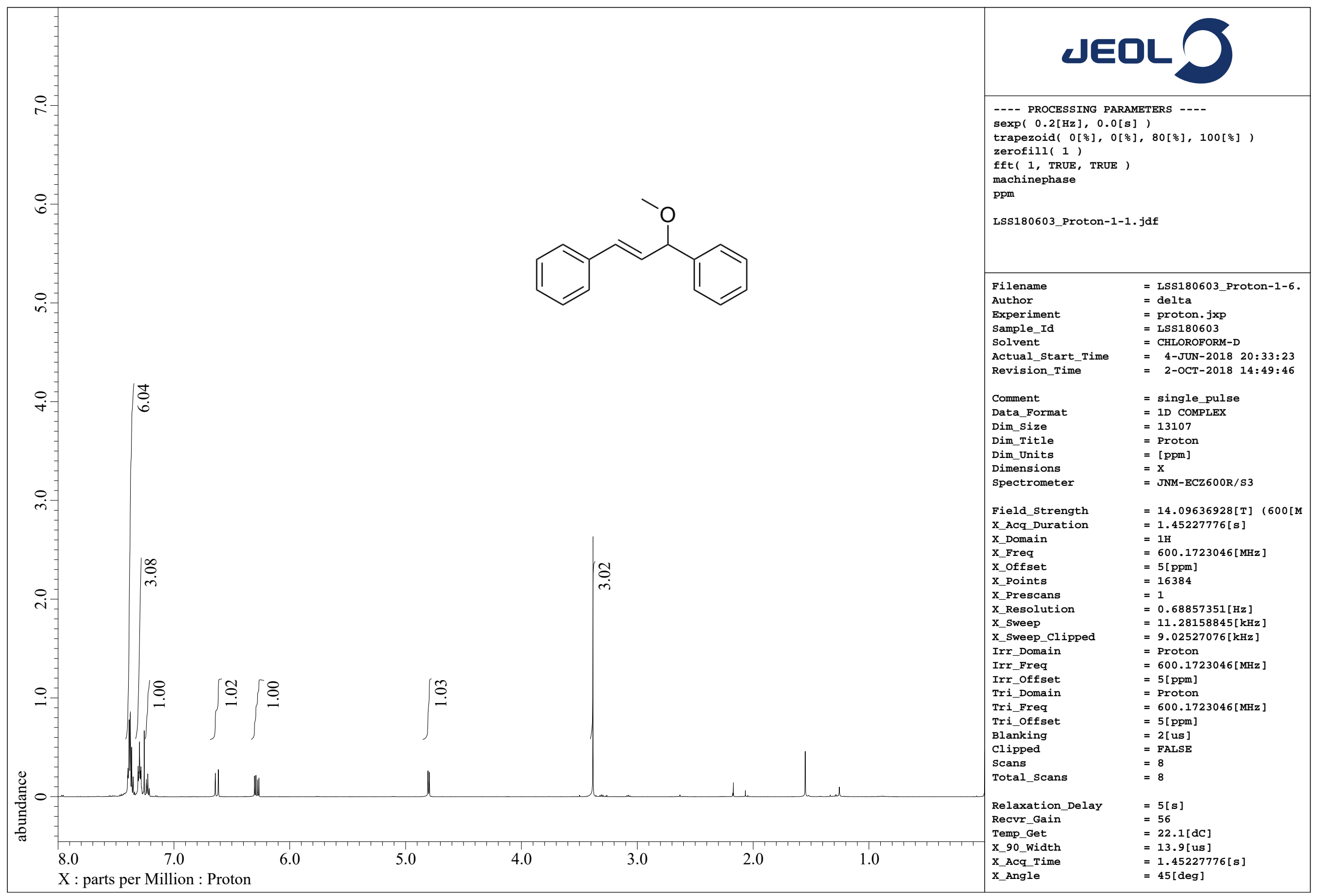




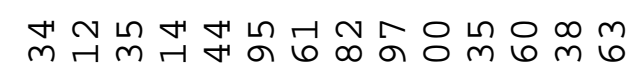

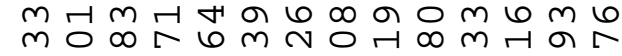
m $\sim \sim N \sim N$ N

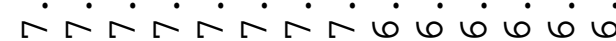
$\longrightarrow$
ํํㄴ

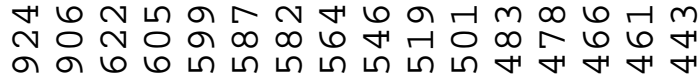

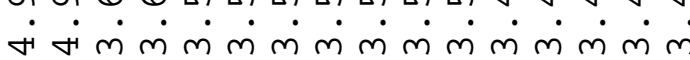

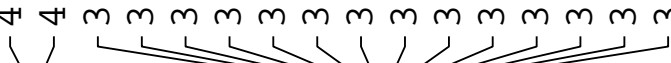

N 0

ก

i

/

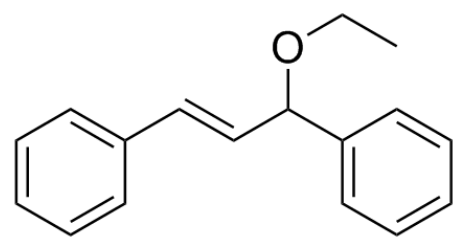

NAME

EXPNO

PROCNO

Date

Time

INSTRUM

PROBHD

PULPROG

TD

SOLVENT

DS

SWH

FIDRES

AQ
RG

RG

DE

TE

TDO

$===$

NUC1

PL1

PL1W

SF01

SI

SI
SF
WDW

SSB

LB

GB

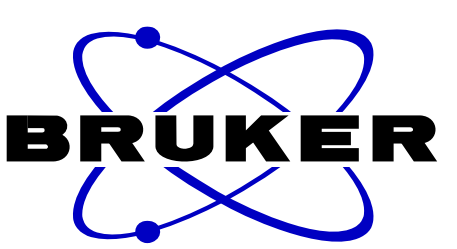

LSS180922- 5

20180925 10.37 $5 \mathrm{~mm}$ РABBO BB$\mathrm{zg} 30$ 65536 CDCl3 0

16163.793 Hz $0.246640 \mathrm{~Hz}$ $2.027297 \odot \mathrm{sec}$ 90.5 30.933 usec $9.2 \mathrm{~K}$

$1.000 \odot \odot \odot \odot \odot \mathrm{sec}$

$=$ CHANNEL $\mathrm{f}$

$======$
$-1.00 \mathrm{~dB}$ $13.18669796 \mathrm{~W}$ $400.1728012 \mathrm{MHz}$ 32768 $400.1700110 \mathrm{MHz}$ EM $0.30 \mathrm{~Hz}$

0
1.00
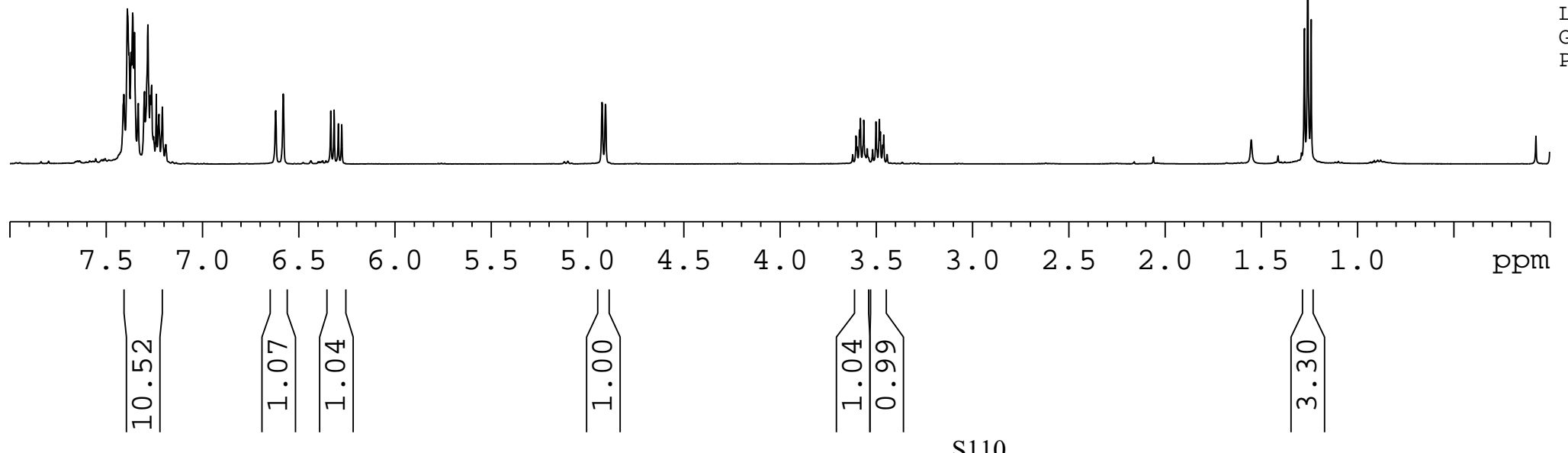
ำ

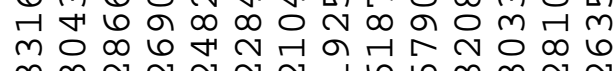

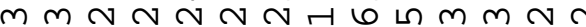
antantago0000

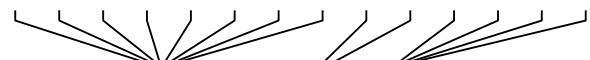

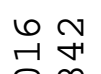

8 $\dot{\gamma} \dot{\gamma}$ i
궁 궁 ก м м м м м м м м त $\longrightarrow+1$
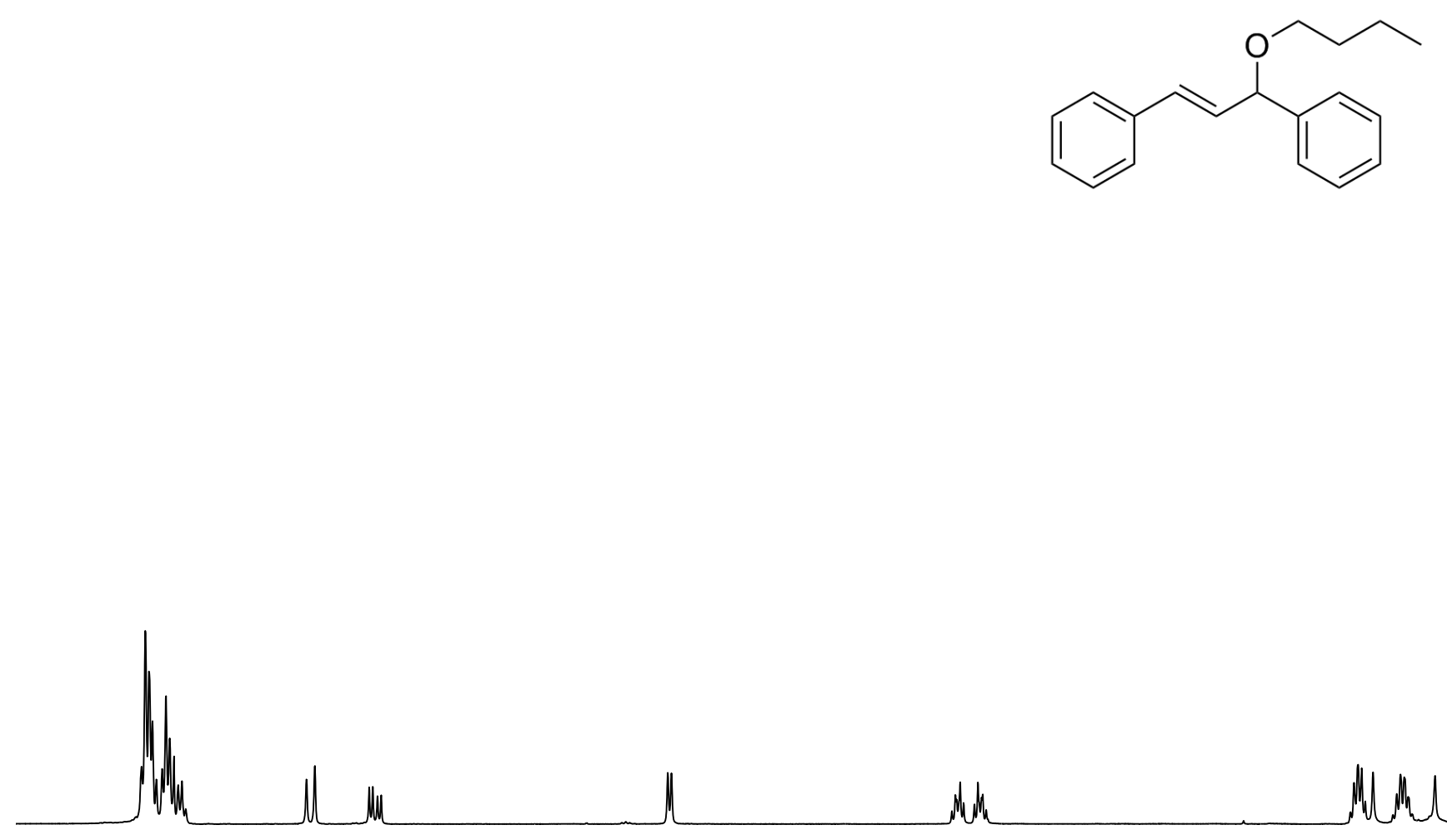

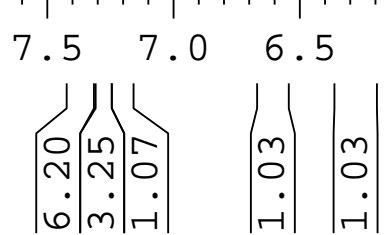

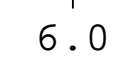

5.5

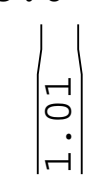

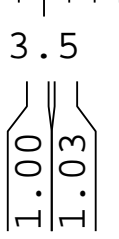

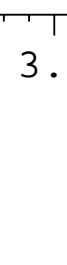

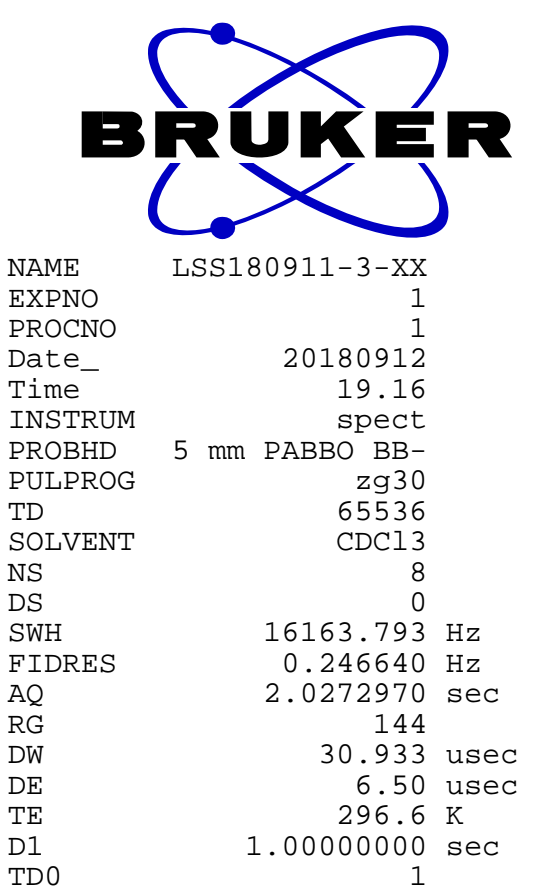

$=====$ NUC1

PL1

PL1W

SF01

SF

WDW

SSB

LB

GB

CHANNEL

f1 $=======$

15.50 usec

$-1.00 \mathrm{~dB}$

$13.18669796 \mathrm{~W}$

$400.1728012 \mathrm{MHz}$

32768

400.1700076 MHZ

EM

$0.30 \mathrm{~Hz}$

$1.0 \odot$ 


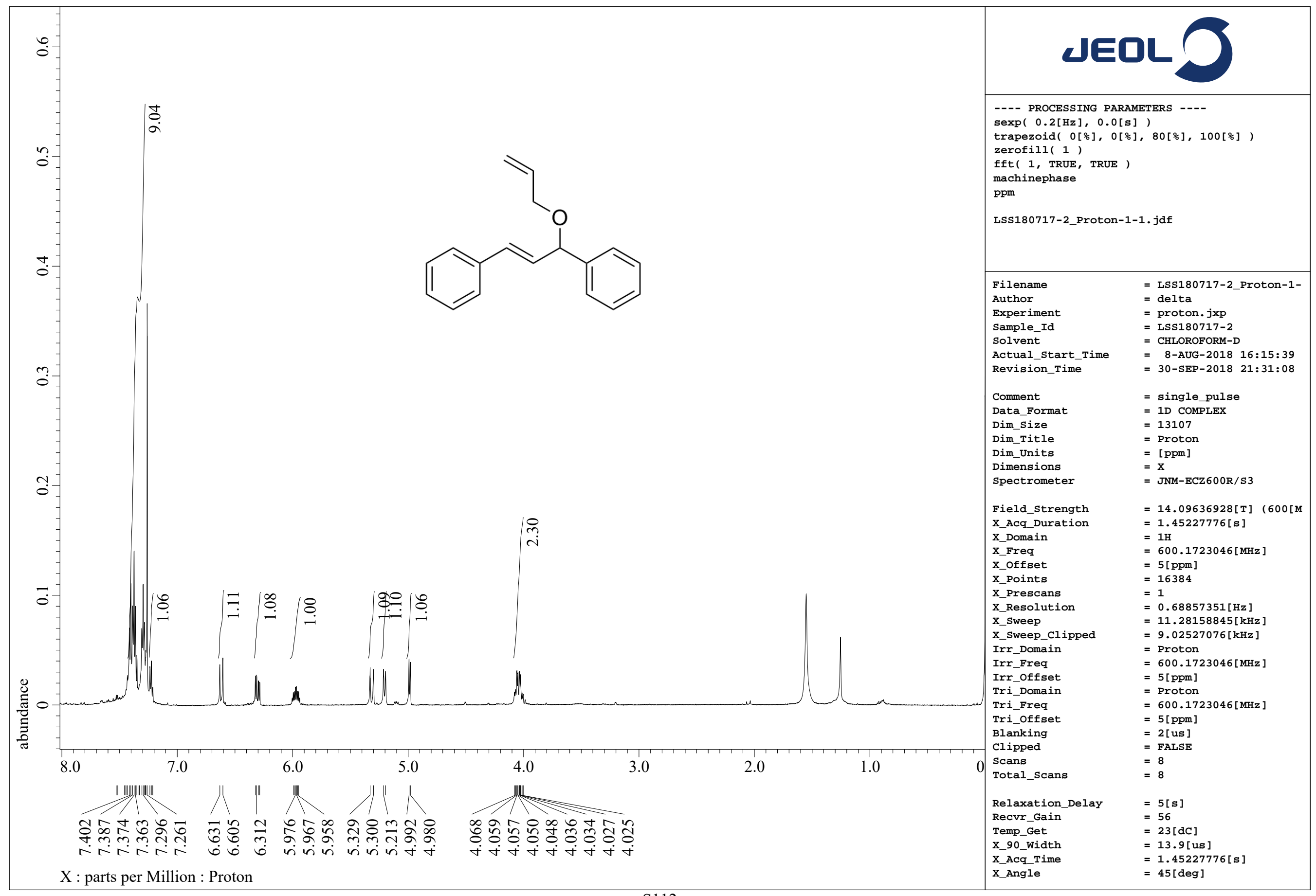




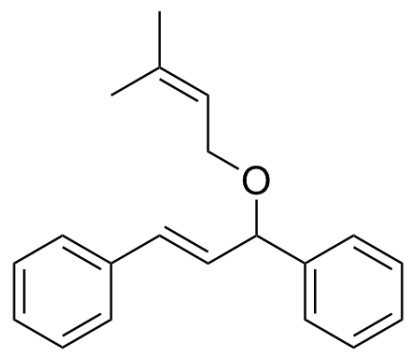

SOLVENT

NS

DS

SWH
FIDRES

AQ

RG

DW

DE

D1

$\overbrace{L S S 180917-6-X X}$

LSS180917 - 6 - XX

20180919

$5 \mathrm{~mm}$ spect

$====$ $\mathrm{NUC}$

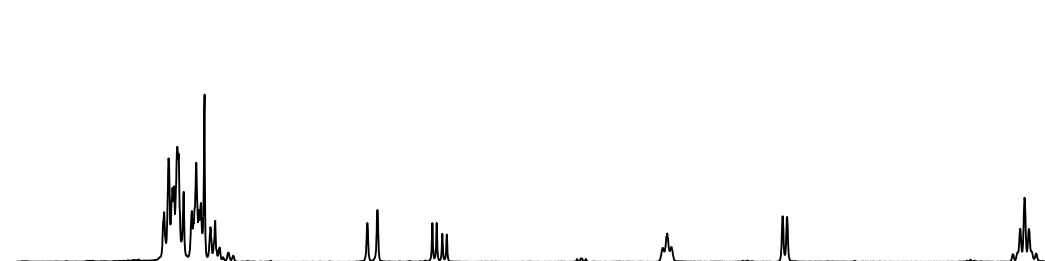

zg30

CDCl3

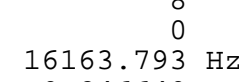

$16163.793 \mathrm{~Hz}$
$0.246640 \mathrm{~Hz}$ $2.0272970 \mathrm{sec}$ 203

30.933 usec 6.50 use

$1.00000000 \mathrm{sec}$ 1

1
$\mathrm{sec}$

$=$ CHANNEL $\mathrm{f}$

$======$
$-1.00 \mathrm{~dB}$
$13.18669796 \mathrm{~W}$ 400.1728012 MHz 32768 $400.1700055 \mathrm{MHz}$ EM $0.30 \mathrm{~Hz}$ 0
$1.0 \odot$

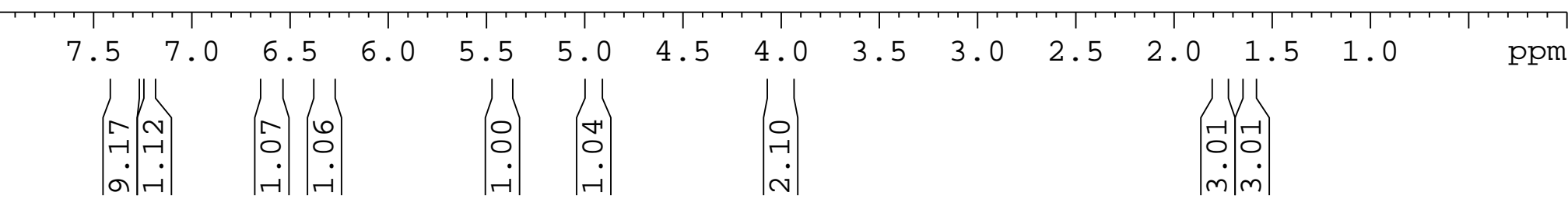


ब

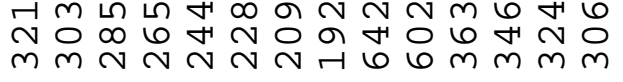
ن

on
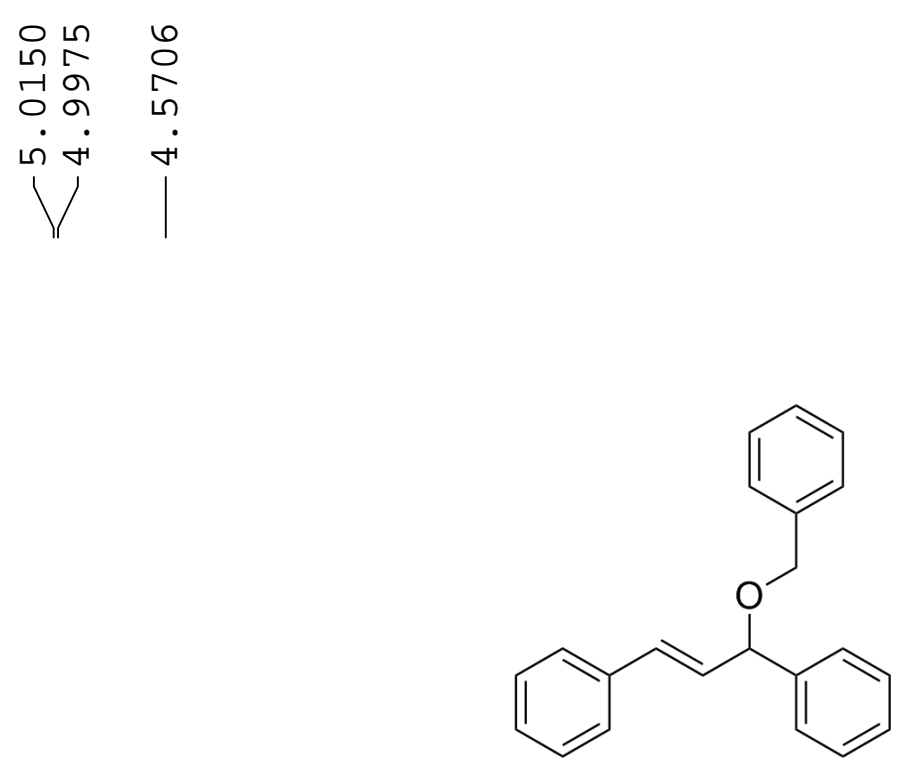

NAME

EXPNO

PROCNO

Date

INSTRUM

PROBHD

PULPROG

TD

SOLVENT

DS

SWH

FIDRES

AQ

RG

$\mathrm{DW}$
$\mathrm{DE}$
$\mathrm{TE}$

TE

D1

\section{$=-=$} NUC1

P1

PL1

SF01

SF01
SI
SF
WDW

WDW

LB

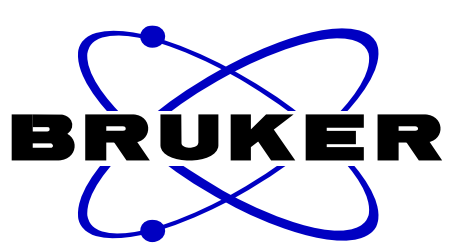

LSS180926 - 4

20180926 17.13 $5 \mathrm{~mm}$ РABBO BB$\mathrm{zg} 30$ 65536 $\mathrm{CDCl}$

$\ominus$
$16163.793 \mathrm{~Hz}$ $0.246640 \mathrm{~Hz}$ $2.0272970 \mathrm{sec}$ 80.6 30.933 usec
6.50 usec

$1.0000 \odot \odot \odot \odot \mathrm{sec}$

$=$ CHANNEL $\mathrm{f}$

$1=======$ 5. 50 usec $15.50 \mathrm{~dB}$ $13.18669796 \mathrm{~W}$ $400.1728012 \mathrm{MHz}$ 32768 $400.1700238 \mathrm{MHz}$ $\mathrm{EM}$
$\odot$
$\odot .30 \mathrm{~Hz}$ 0.30
0
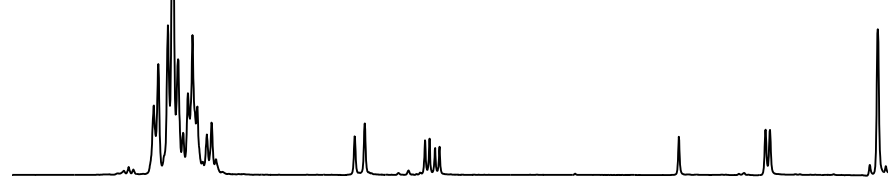


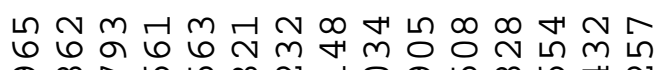

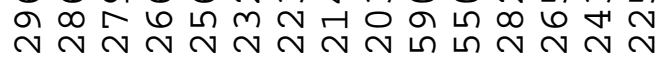

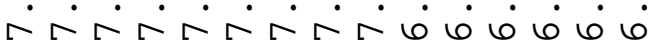

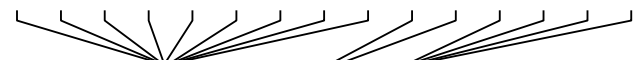

시유

ब त

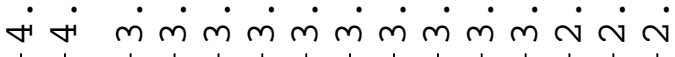
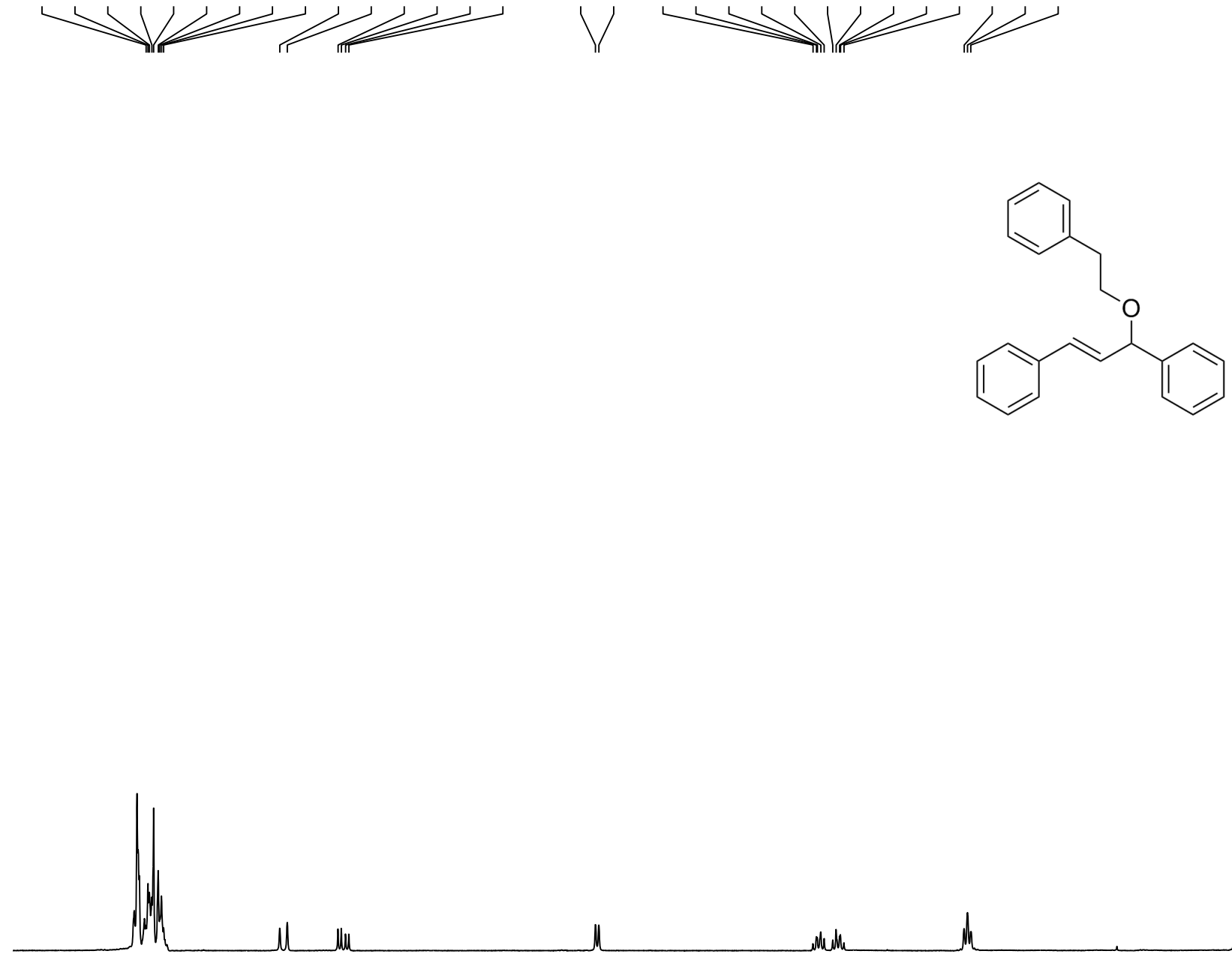

Mulk

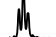

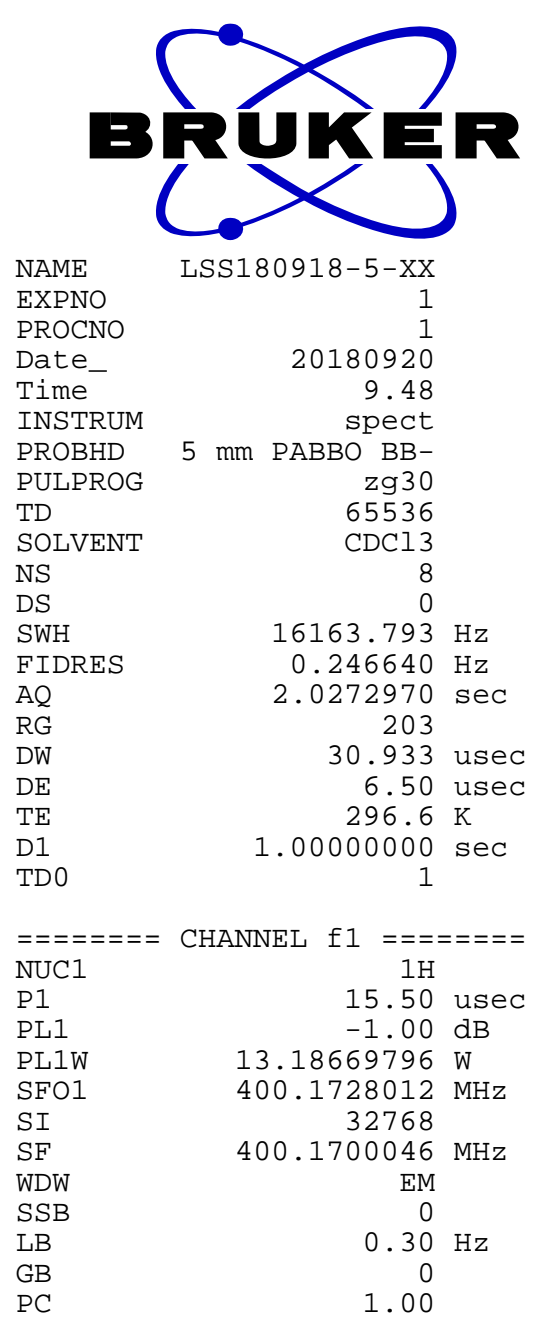

$\begin{array}{lllllllllllllllll}7.5 & 7.0 & 6.5 & 6.0 & 5.5 & 5.0 & 4.5 & 4.0 & 3.5 & 3.0 & 2.5 & 2.0 & 1.5 & 1.0 & \mathrm{ppm}\end{array}$

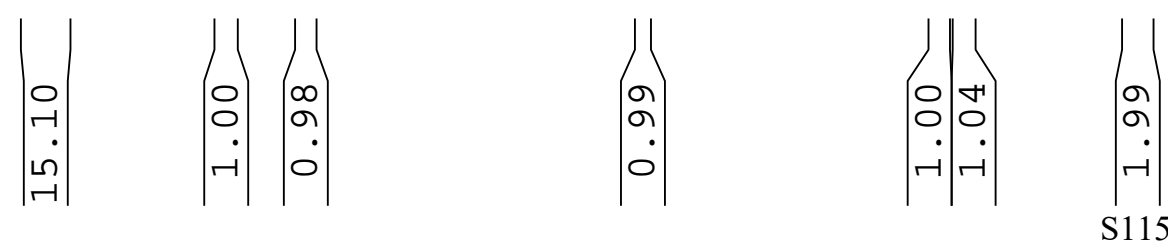


बㄱำ $\odot$ ब ल ن $31 / 11$
요 $\quad m_{n \infty}^{\infty}$

นᄂ้จ

ம்

V
ำ

$\dot{4} \dot{0}$
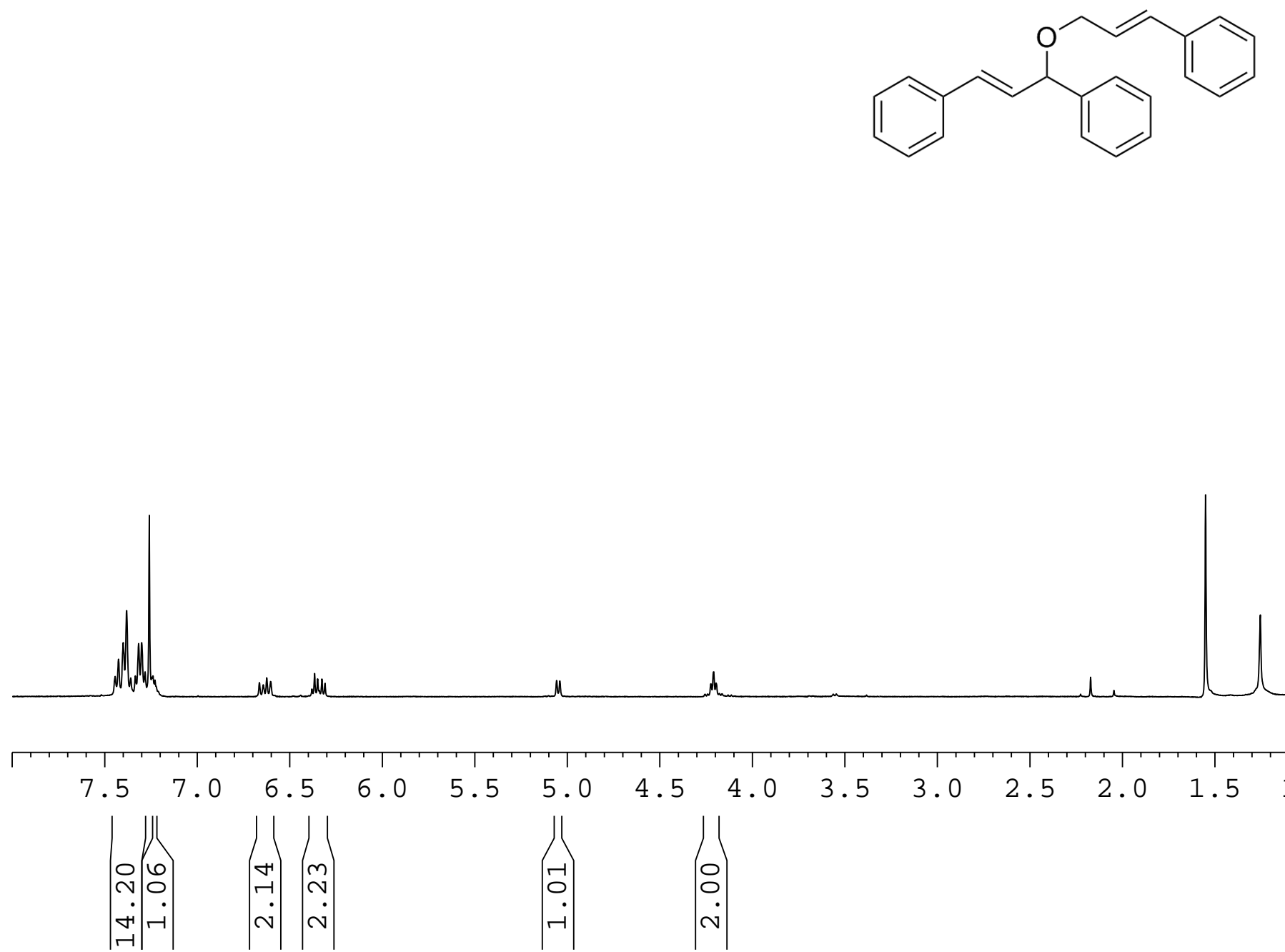

5.5

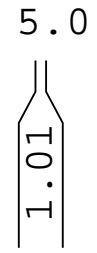

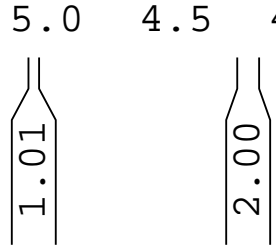

ppm 


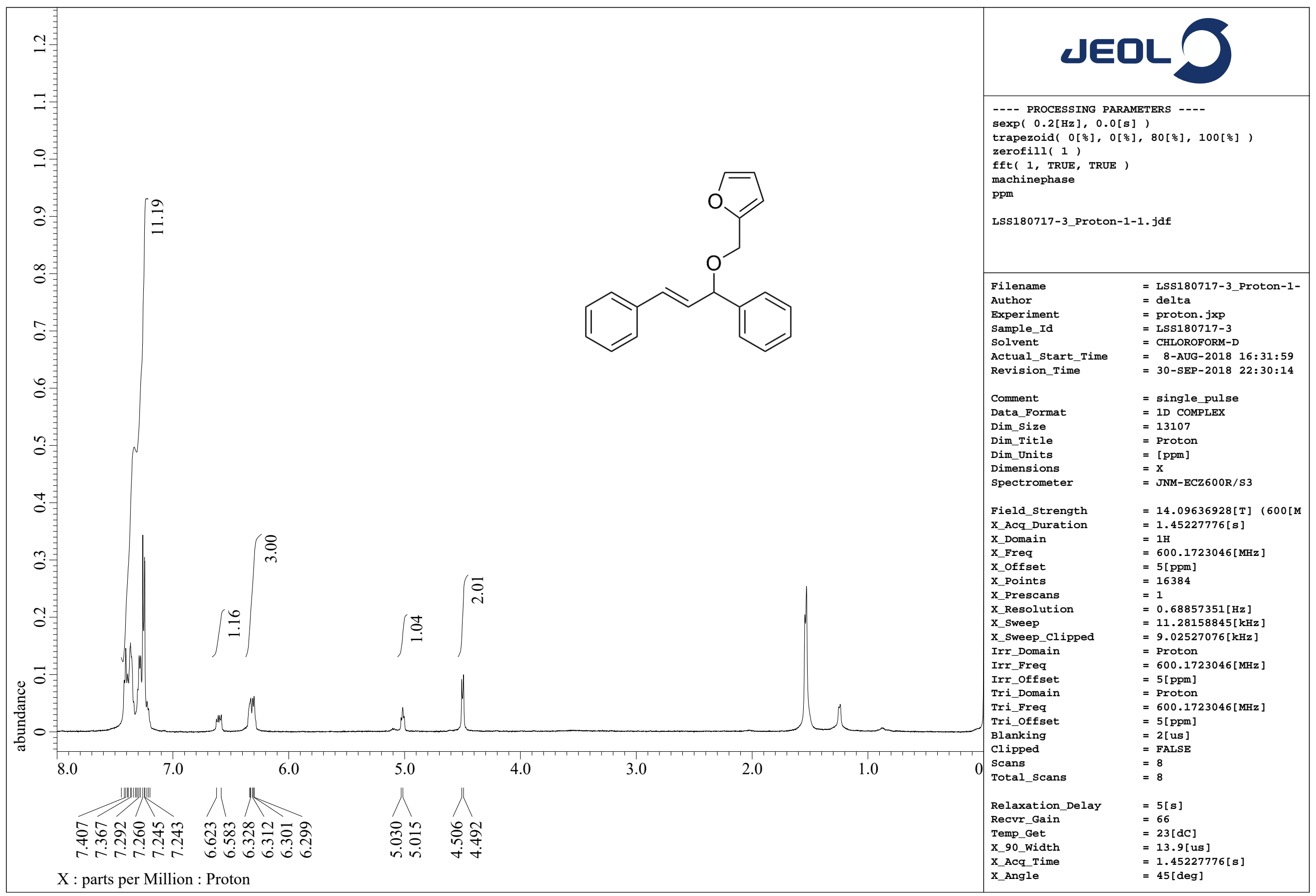



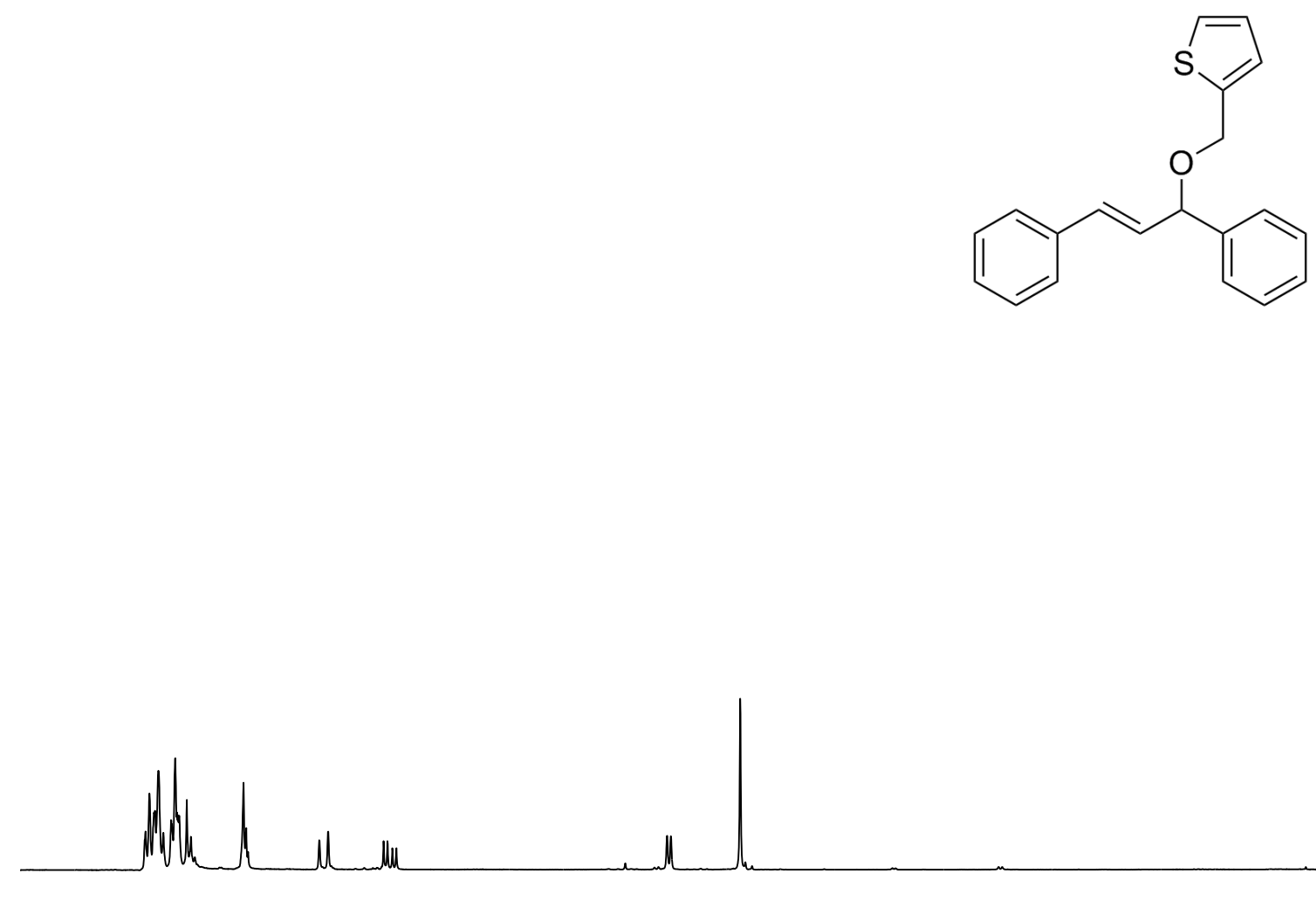

$\begin{array}{llll}7.5 & 7.0 & 6.5 & 6.0\end{array}$

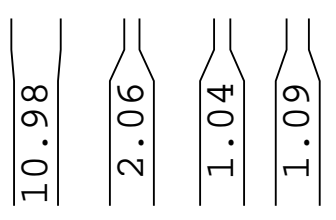

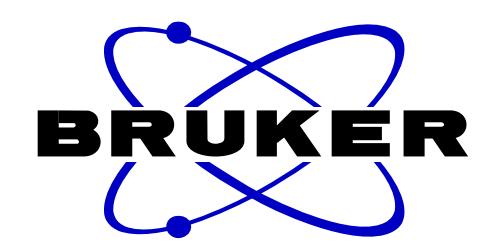

NAME

EXPNO

PROCNO

Date

Time-

INSTRUM

PROBHD

PULPROG

TD

SOLVENT

NS
DS
SWH

SWH
FIDRES

AQ

RG

$\mathrm{DW}$

DE

D1

LSS180927-3

20180927

16.19

$5 \mathrm{~mm}$ РABBO BB-

zg30

65536
CDC13

8
$\odot$
$16163.793 \mathrm{~Hz}$

$16163.793 \mathrm{~Hz}$
$0.246640 \mathrm{~Hz}$

$2.027297 \odot \mathrm{sec}$ 161

30.933 usec

6.50 usec

$1.0000000 \bigcirc$
1

$=======$ CHANNEL $\mathrm{f}$

NUC1

P1

PL1W

SF01

SI

SF

WDW

LB

$\mathrm{GB}$
$1 \mathrm{H}$

15.50 use

$-1.0 \odot \mathrm{dB}$
$13.18669796 \mathrm{~W}$

$400.1728012 \mathrm{MHz}$

32768
400.1700094

$94 \mathrm{MHz}$

EM

$\odot .30 \mathrm{~Hz}$

1.00

ppm 


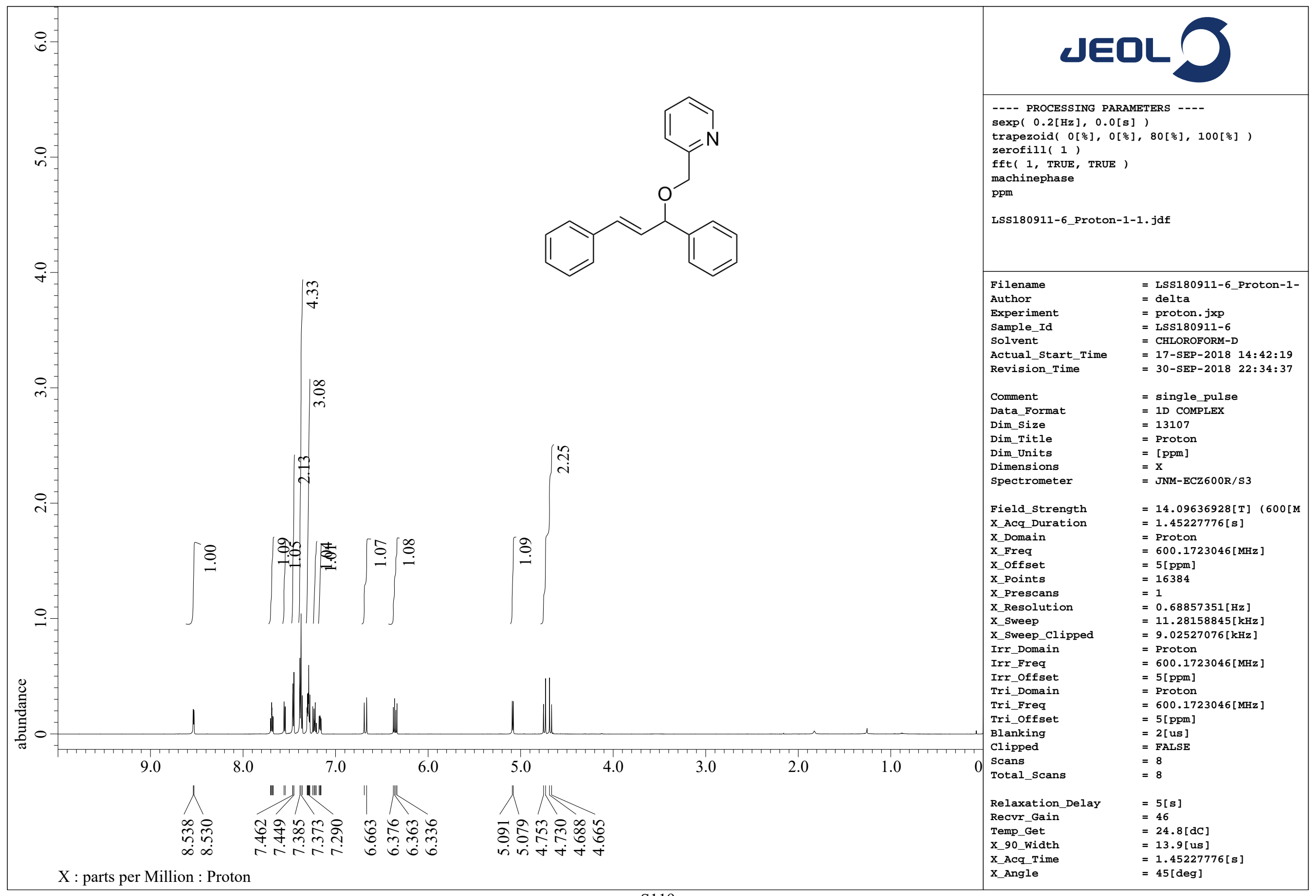




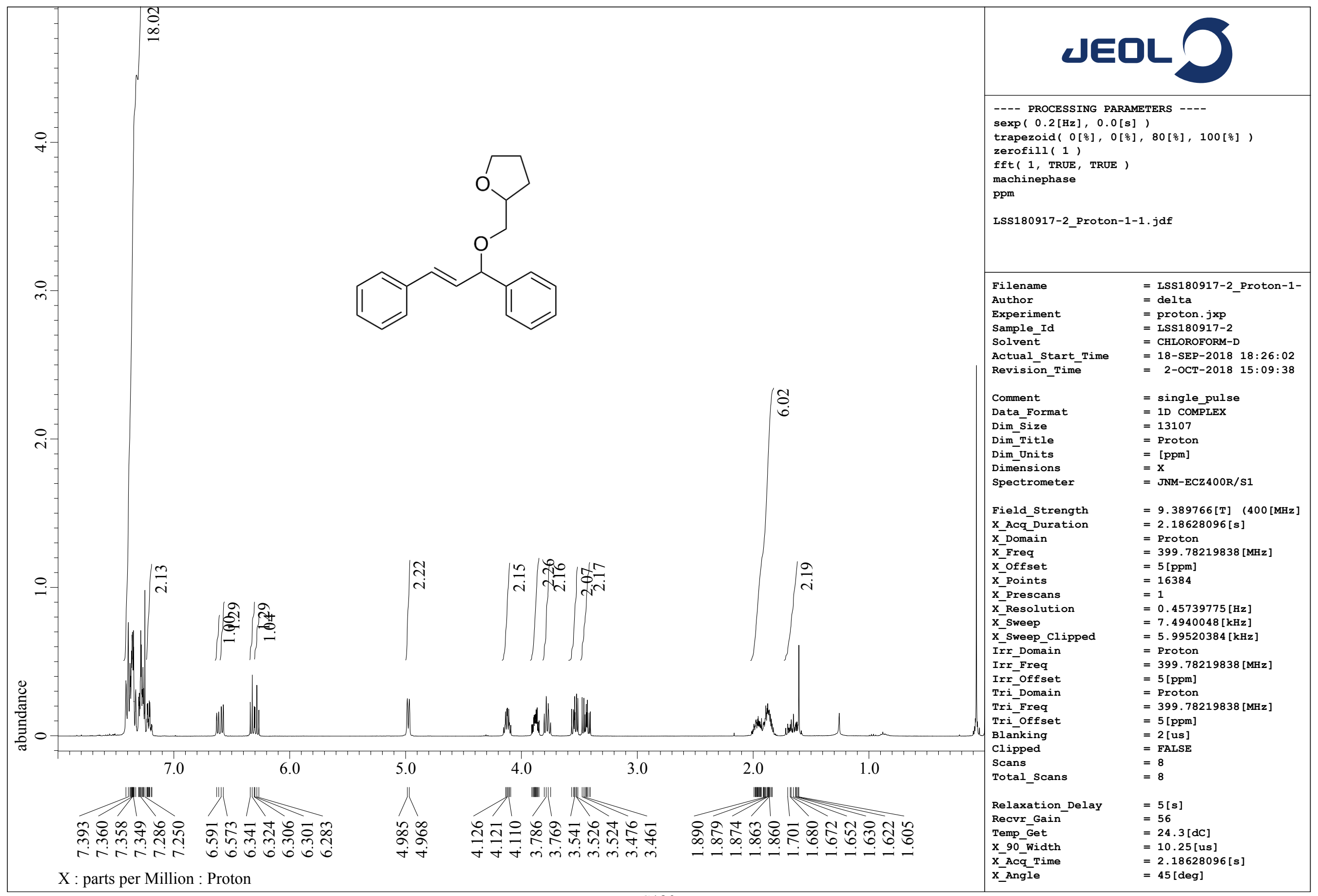




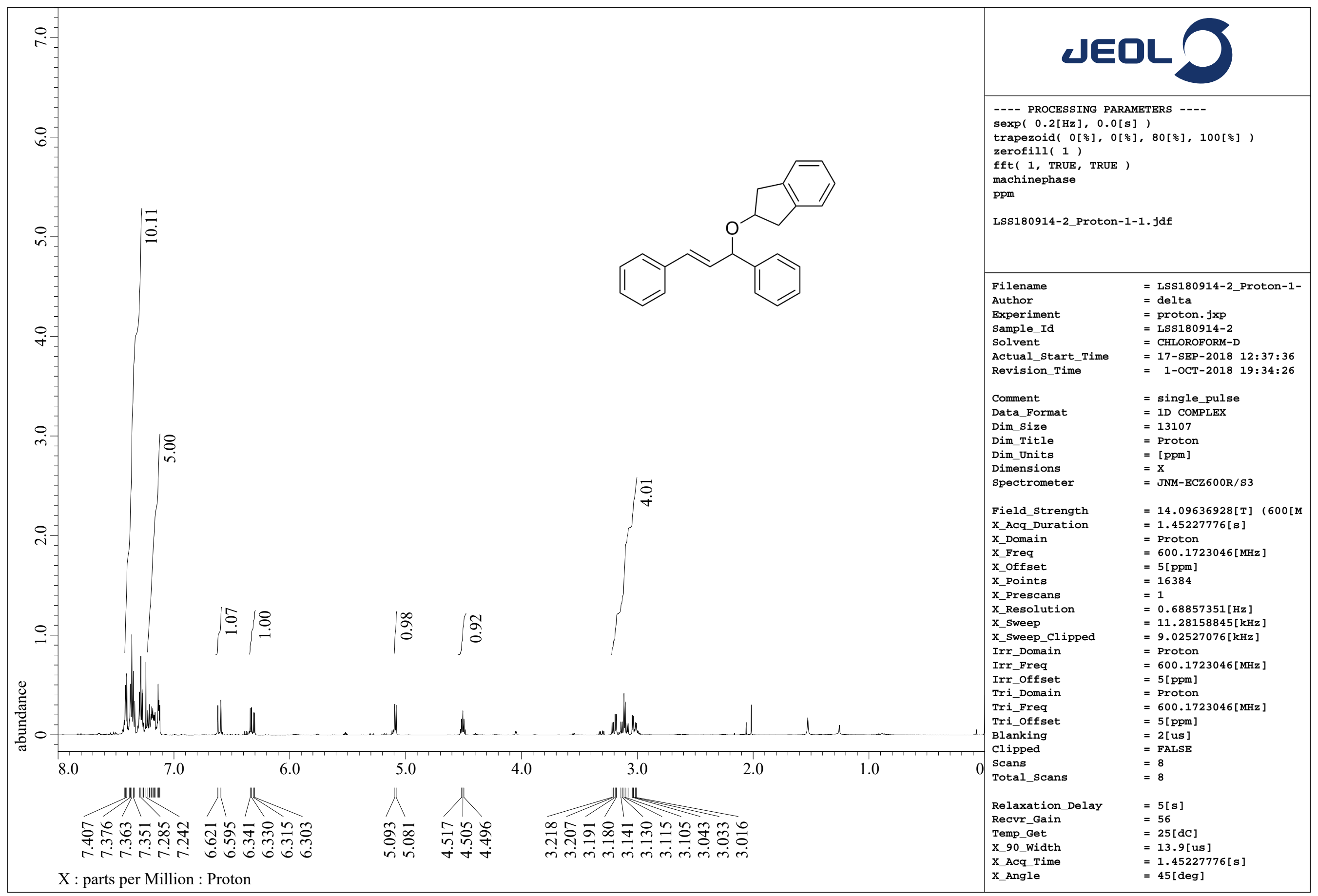




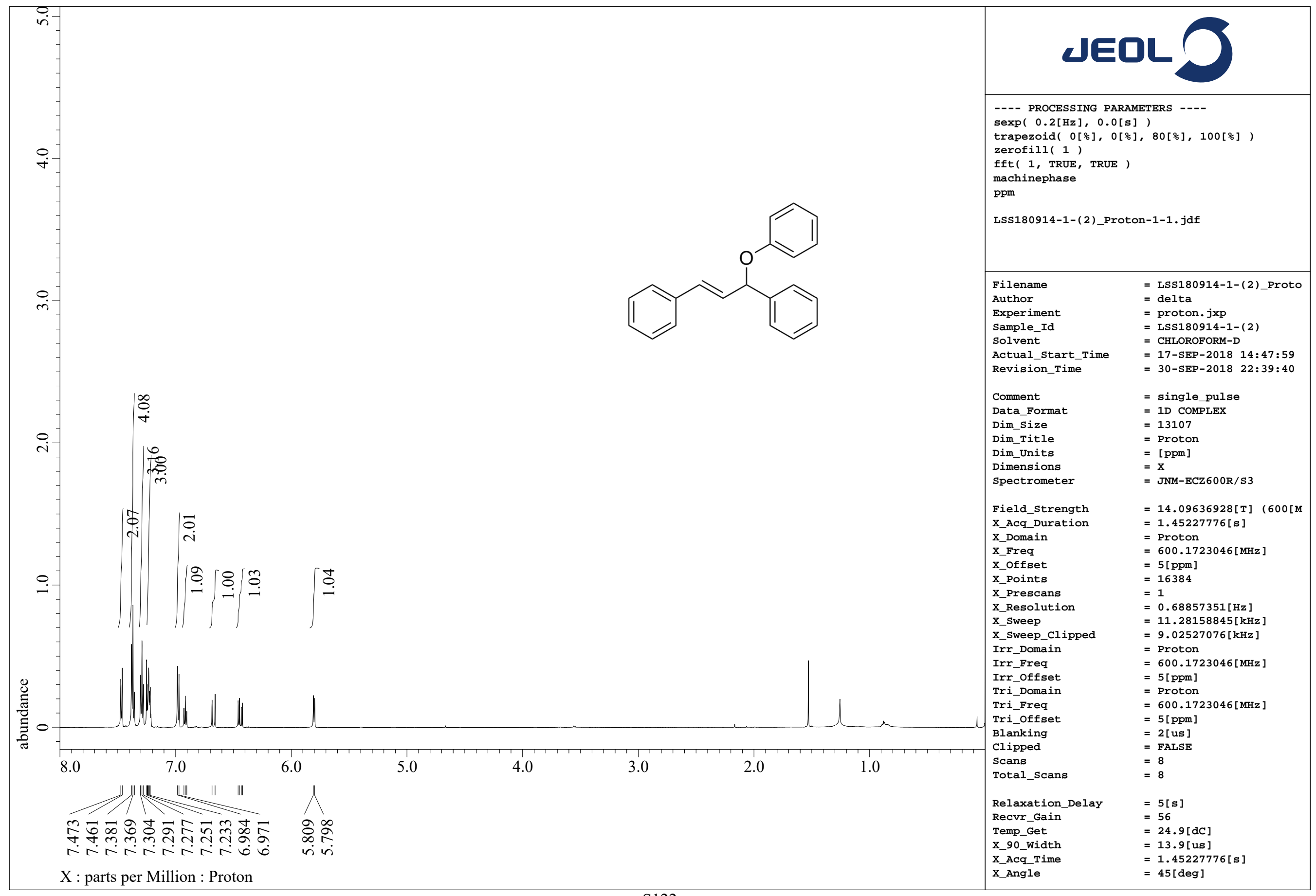



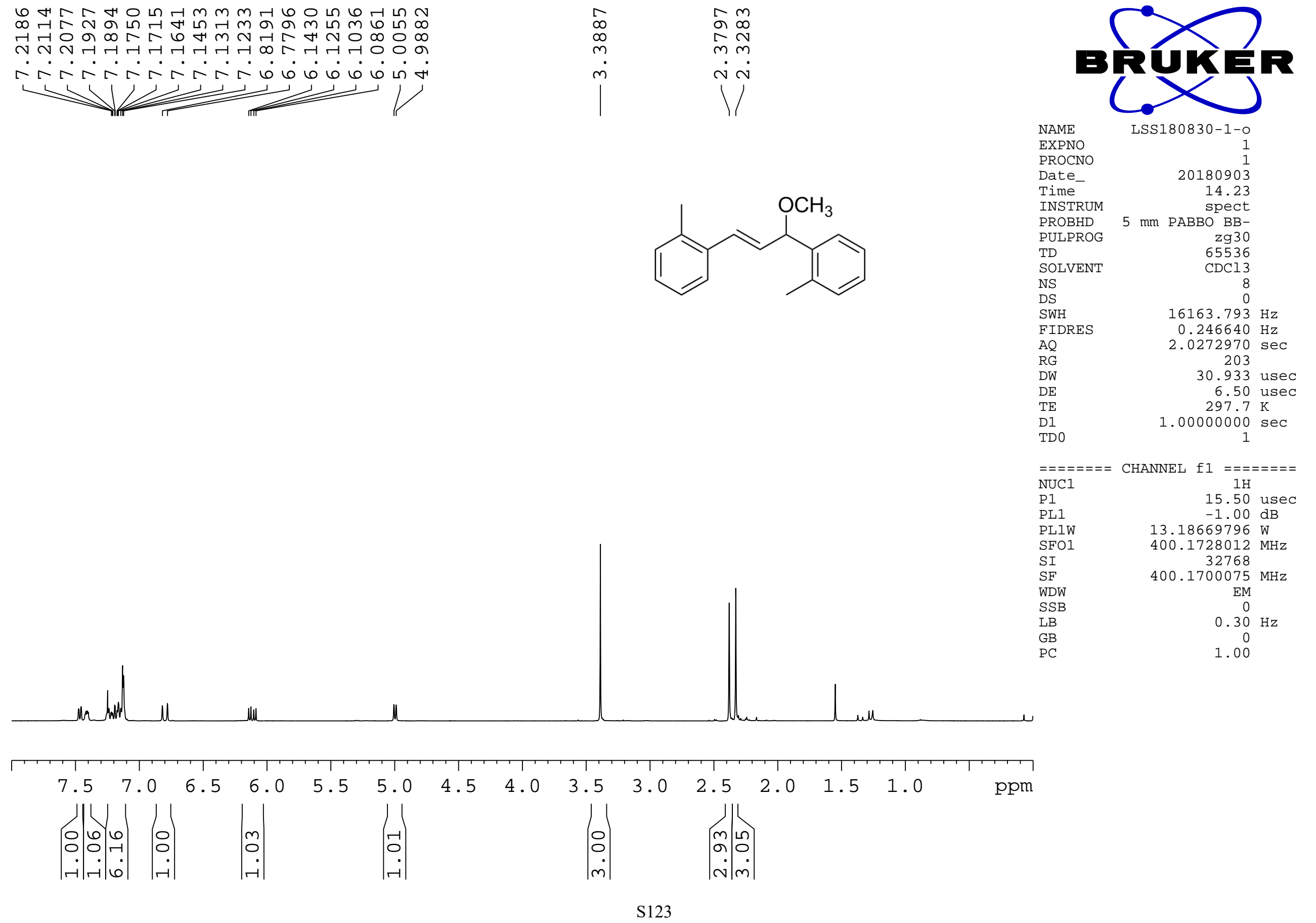

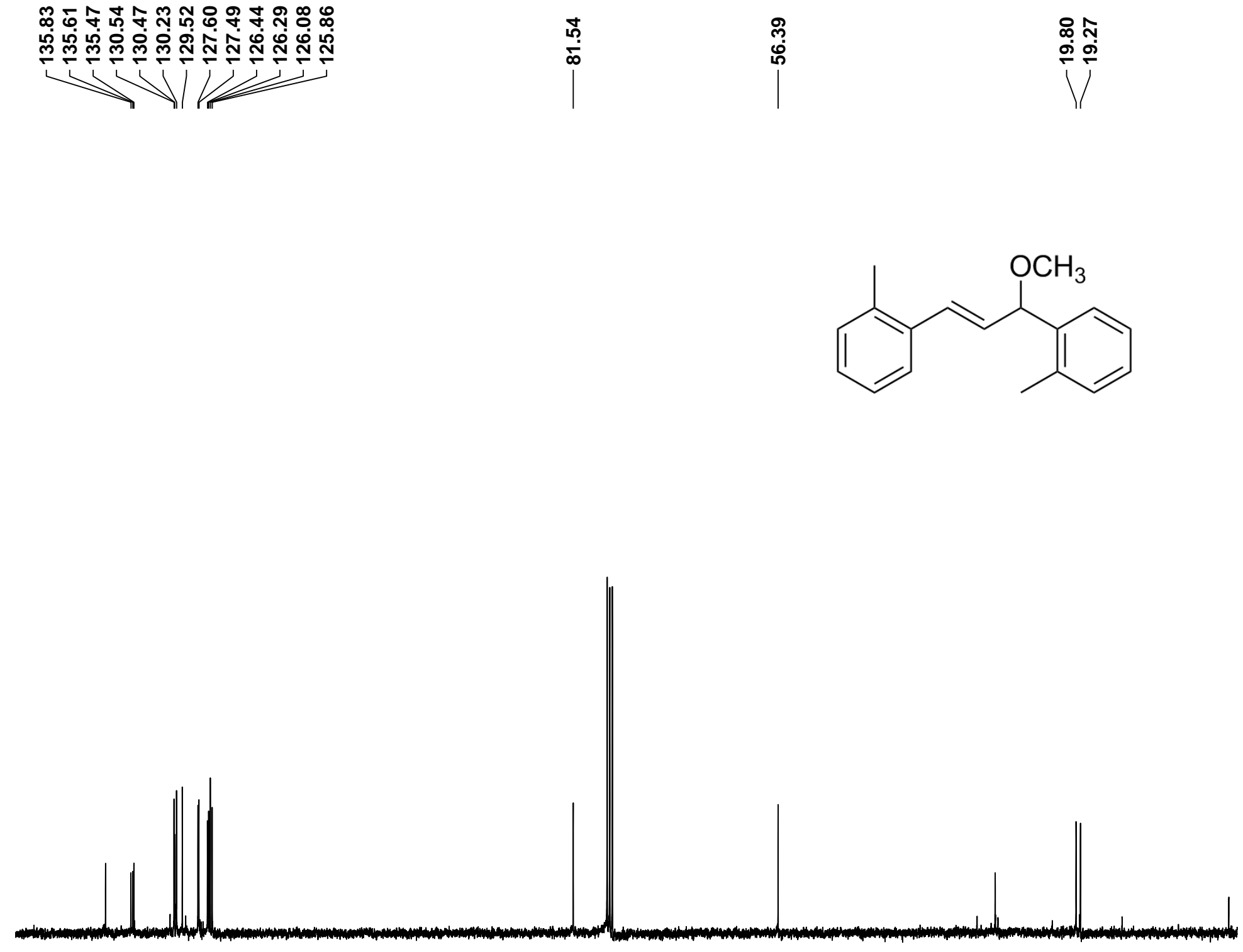

$\begin{array}{llllllllllllllll}140 & 130 & 120 & 110 & 100 & 90 & 80 & 70 & 60 & 50 & 40 & 30 & 20 & 10 & \mathrm{ppm}\end{array}$


๙ N ó $<11$ $\longrightarrow+1$

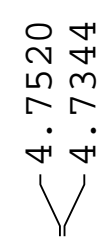

$N$
$\infty$
0
$m$

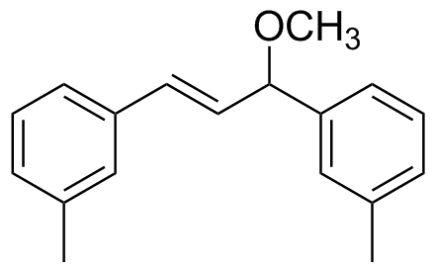

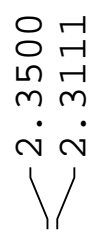
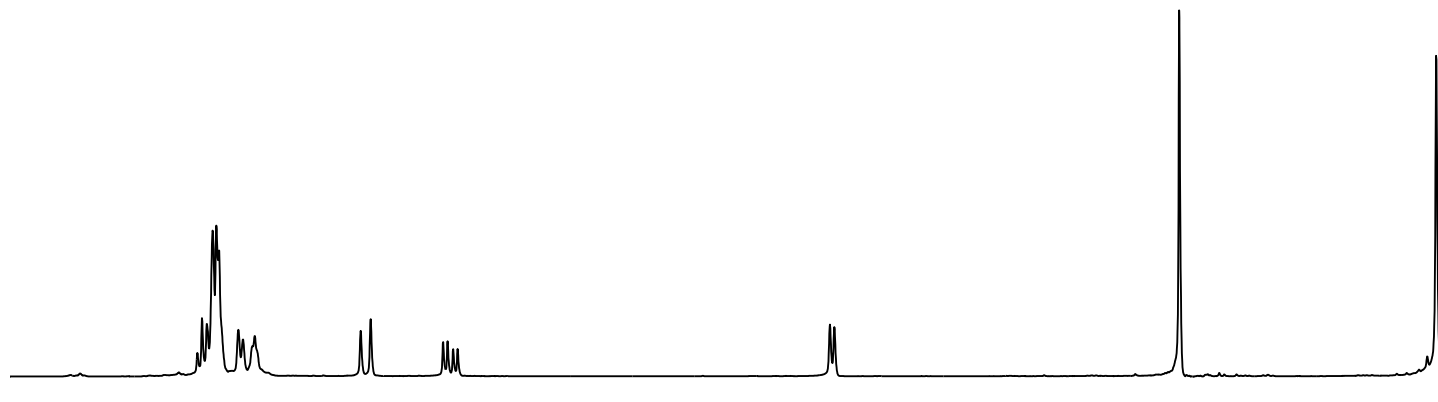

BPUKER ,

NAME

EXPNO

PROCNO

Time

LSS180828-2-m-XX-C13

PULPROG

SOLVENT

NS

NS

SWH

FIDRES

AQ

RG

RG
DW
DE

DE

D1

TDQ

$=======$ CHANNEL f1 ======== NUC1

$16163.793 \mathrm{~Hz}$

$0.246640 \mathrm{~Hz}$

$$
\begin{array}{r}
71.8 \\
30.933
\end{array}
$$

30.933 usec 6.50 usec 1. $0000000 \bigcirc \mathrm{sec}$

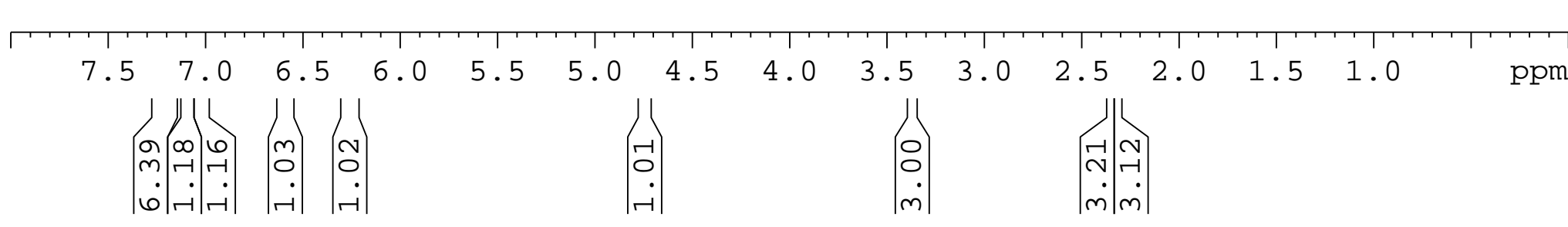


쇼용ํำ 뉴 ๑

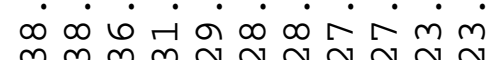

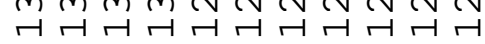
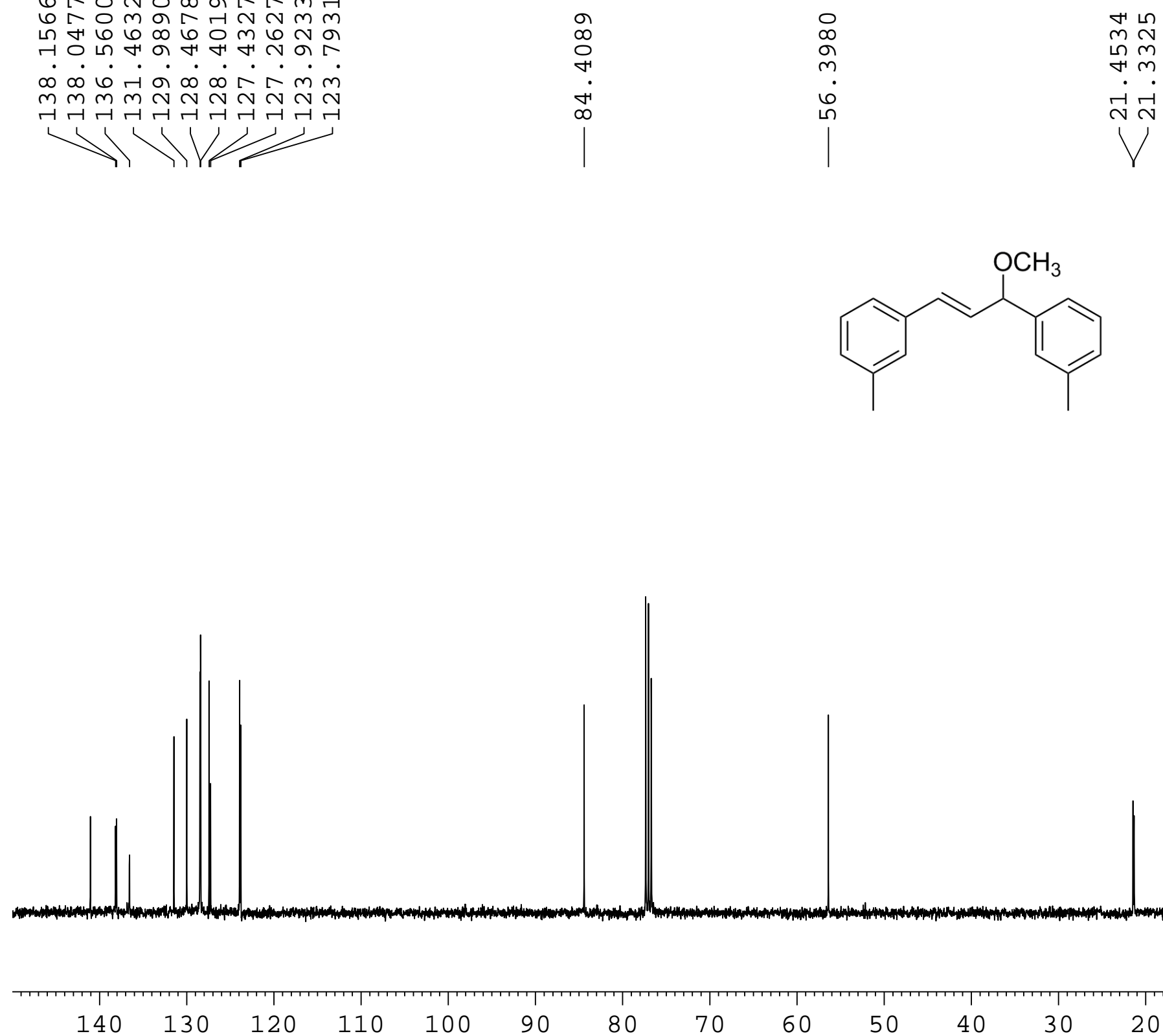

80

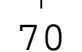

60

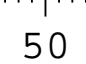

40

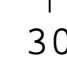

20

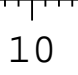

$10 \mathrm{p}$

I SF

SSB

LB
NAME
EXPNO
PROCNO
Time-
INSTRUM
PROBHD
PULPROG
TD
SOLVENT
NS
SWH
FIDRES
AQ
RG
DE
TE
D1 11
LSS180828-m-XX-C13
1
20180901
$5 \mathrm{~mm}$ PABBO BB-
zgpg30
65536
CDCl3
138
4
$71428.570 \mathrm{~Hz}$
$1.089913 \mathrm{~Hz}$
$0.4588020 \mathrm{sec}$
203
000 usec
6.50 usec
$298.0 \mathrm{~K}$
$2.00000000 \mathrm{sec}$
$0.03000000 \mathrm{sec}$
TD 1

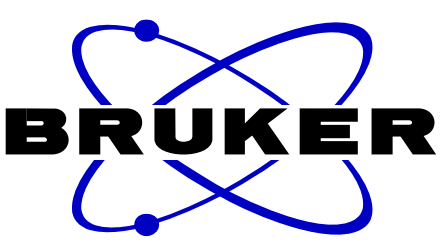

$=======$ CHANNEL $\mathrm{f}$

P1

PL1

$1=$

SF01 $57.32743073 \mathrm{~W}$

$=======$ CHANNEL f2 $=======$

CPDPRG2

NUC2

PCPD2

PL2

PL13

(1)

(13W
waltz16
80.00 usec
$-1.00 \mathrm{~dB}$
$13.26 \mathrm{~dB}$
$14.46 \mathrm{~dB}$
$13.18669796 \mathrm{~W}$
$0.49446553 \mathrm{~W}$
$0.37509048 \mathrm{~W}$
$400.1716007 \mathrm{MHz}$
32768
4.1716

$100.6228270 \mathrm{MHz}$

$100.6228294 \mathrm{MHz}$ EM

0
$1.00 \mathrm{~Hz}$
$\Theta$
$1.4 \odot$ 
ஸ

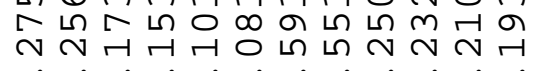

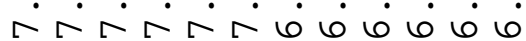
$\longrightarrow 111$
ก

กิ

$N$

$\dot{8}$ mं लेन

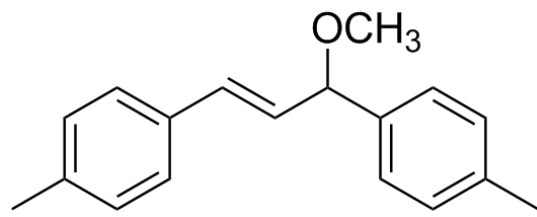

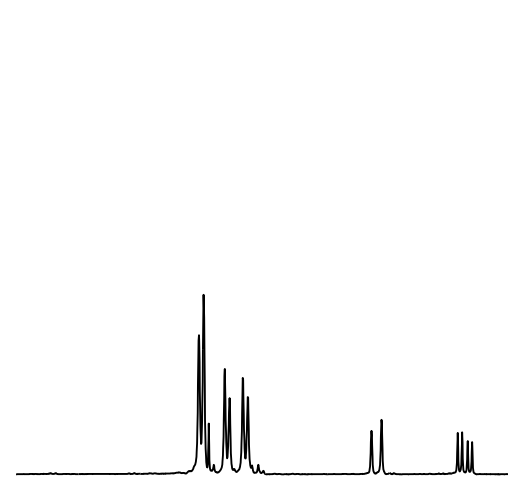
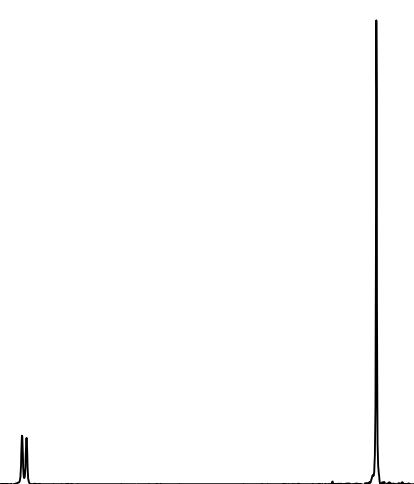

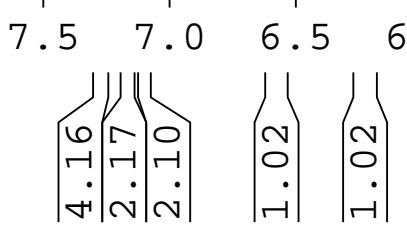

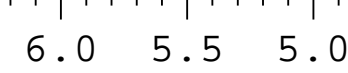

$\left|\begin{array}{r}1 \\ 8 \\ 8 \\ -1\end{array}\right|$
AME

EXPNO

PROCNO

Date

Time

INSTRUM

PROBHD

PULPROG

TD

SOLVENT

DS

DS

FIDRES

AQ
RG

RG

DW

TE

D1

TDO

NUC1

P1
PL1

PL1

SF01

SI
SF
WDW

WDW

LB

GB

PC

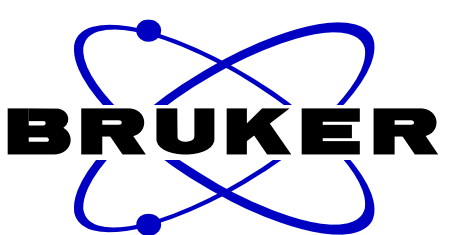

1ss180821-2-c13

20180823

19.28

$5 \mathrm{~mm}$ PABBO BB-

$\mathrm{zg} 30$

65536

$\mathrm{CDCl3}$

16163. $903 \mathrm{~Hz}$

$0.246640 \mathrm{~Hz}$

2. $0272970 \mathrm{sec}$ 114

30.933 usec 6.50 usec

$1.00000000 \mathrm{sec}$ 1 sec

\section{CHANNEL f1 ========}

15.50 usec $-1.00 \mathrm{~dB}$ $13.18669796 \mathrm{~W}$ $400.1728012 \mathrm{MHz}$ 32768 $400.1700123 \mathrm{MHz}$ $\mathrm{EM}$
0 $0.30 \mathrm{~Hz}$ 0
$1.0 \Theta$ 


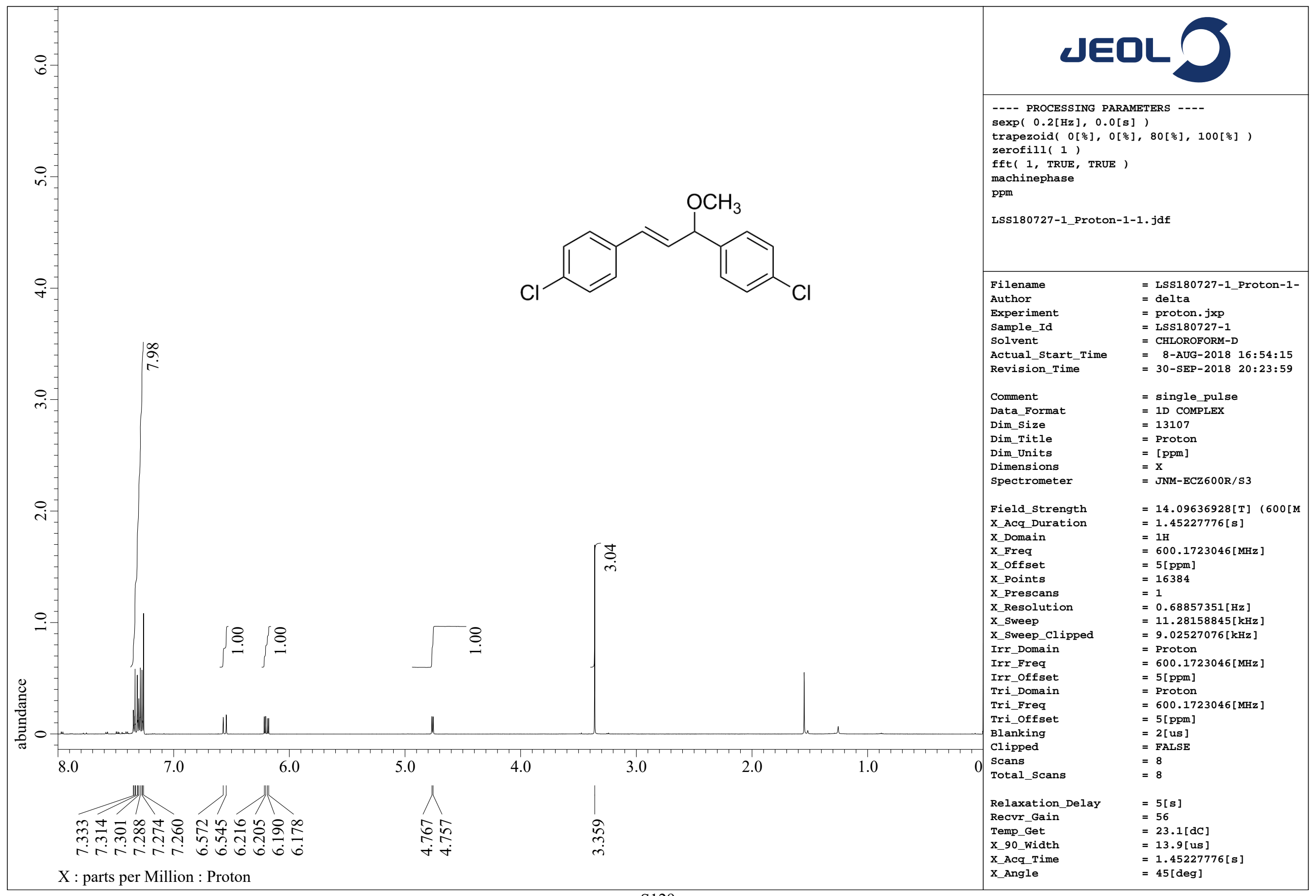




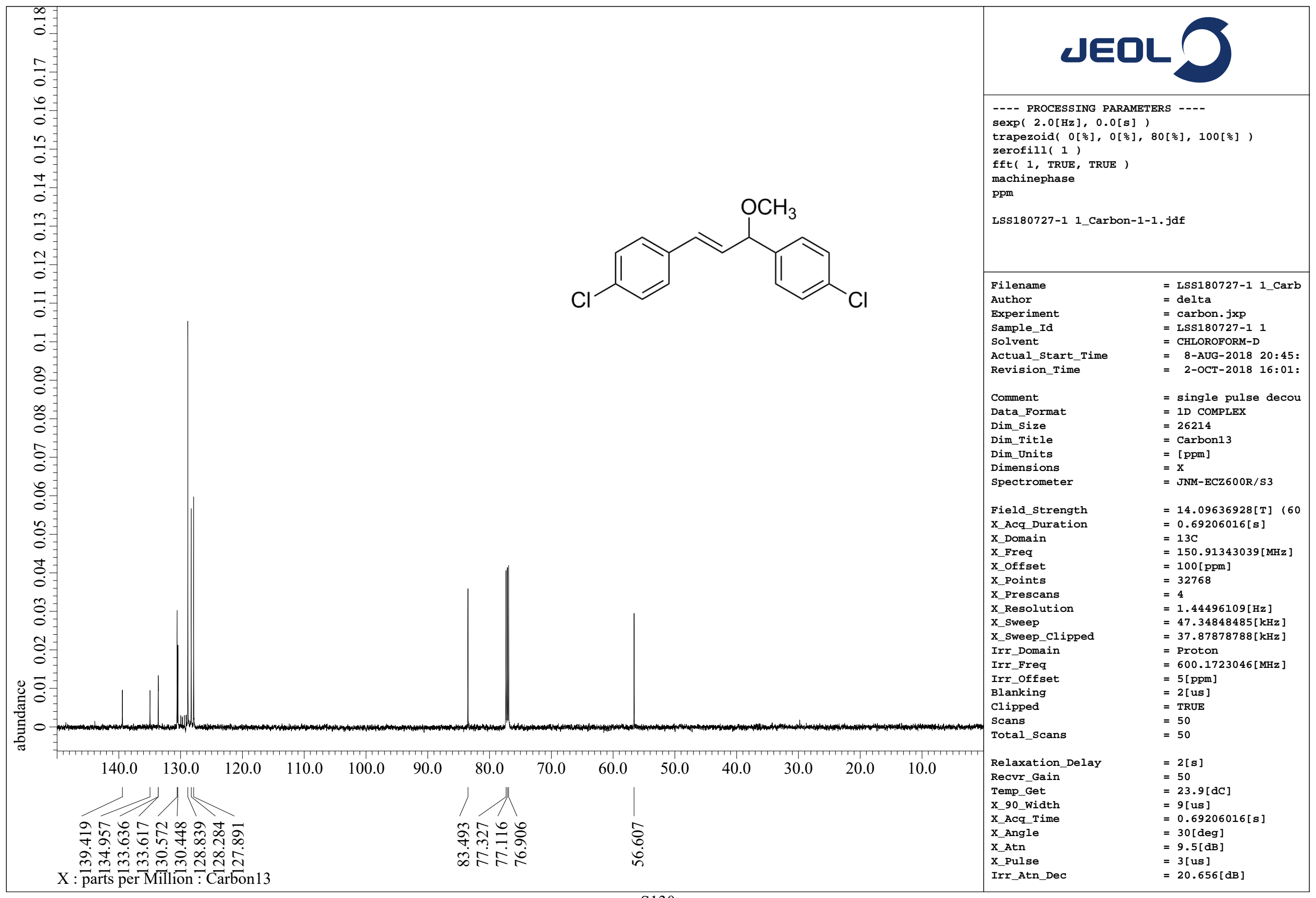




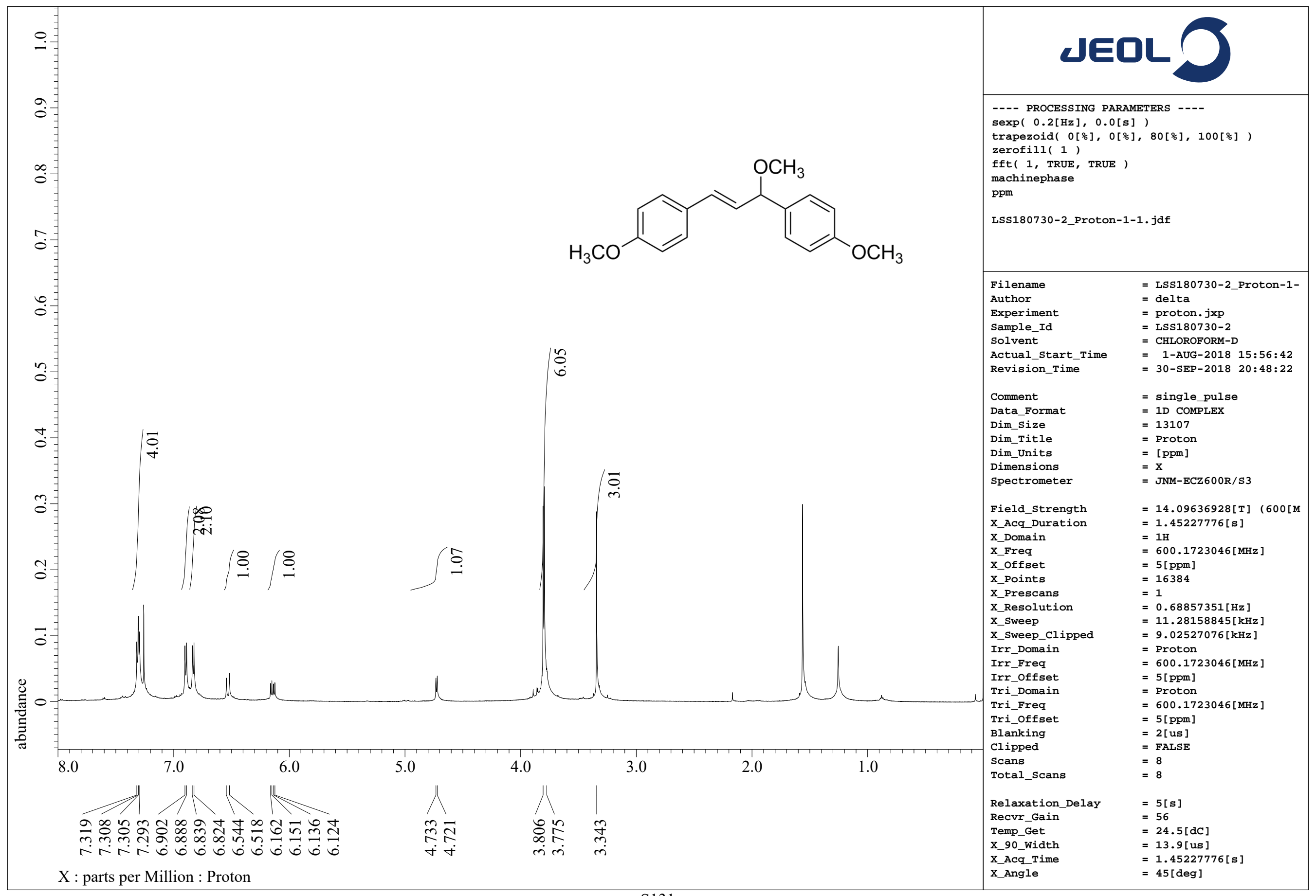




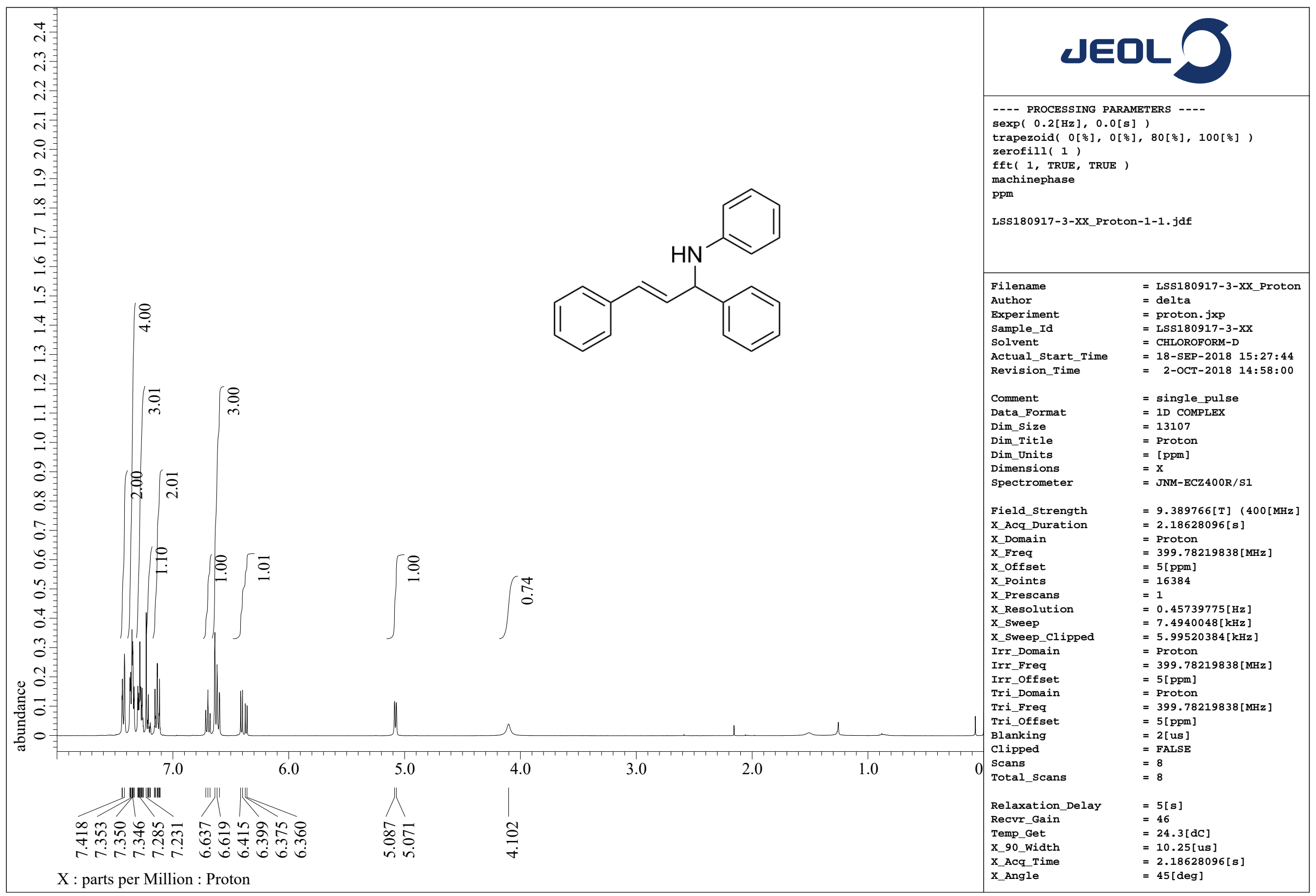




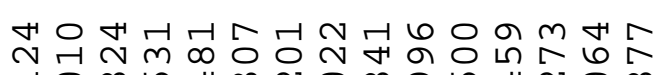

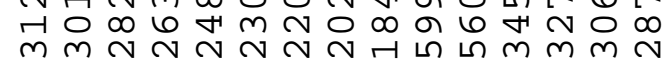
ن

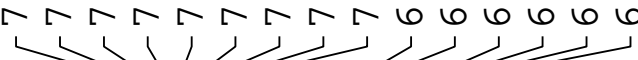
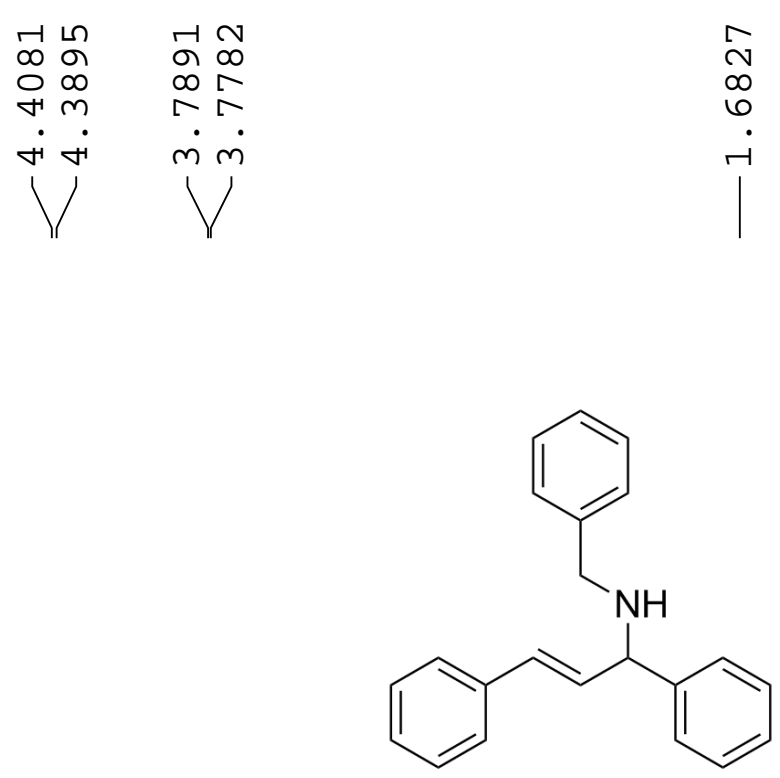

$$
\text { m }
$$$$
\overbrace{}^{-1} \infty
$$

V

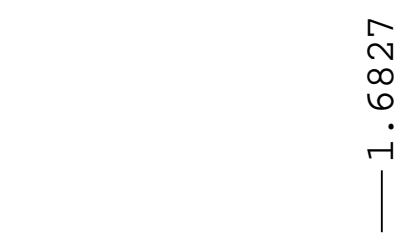

N
0
0
$\dot{1}$
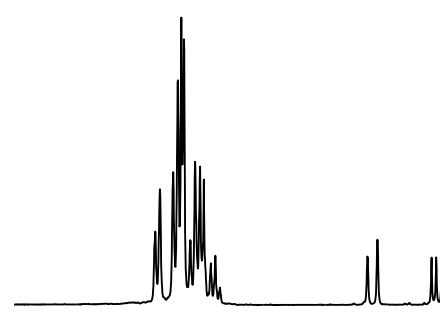

=

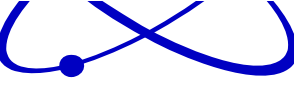

NAME

EXPNO

PROCNO

Date_

TimSTRUM

PROBHD

PULPROG

TD

SOLVENT

LSS180911- 5

20180913

17.47

$5 \mathrm{~mm}$ PABBO BB-

NS

DS

SWH

FIDRES

AQ

RG

DW

TE

D1

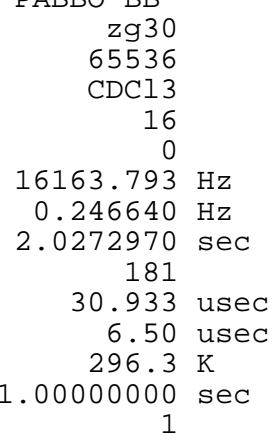

$====$

P1

PL1

SF01

SI

SF

WDW

LB

GB

$=$ CHANNEL $\mathrm{f}$

$1======$

1H 1 usec

$-1.00 \mathrm{~dB}$

$13.18669796 \mathrm{~W}$

$400.1728012 \mathrm{MHz}$

32768

$400.1700079 \mathrm{MHz}$

EM

$\odot .30 \mathrm{~Hz}$

$1.0 \odot$

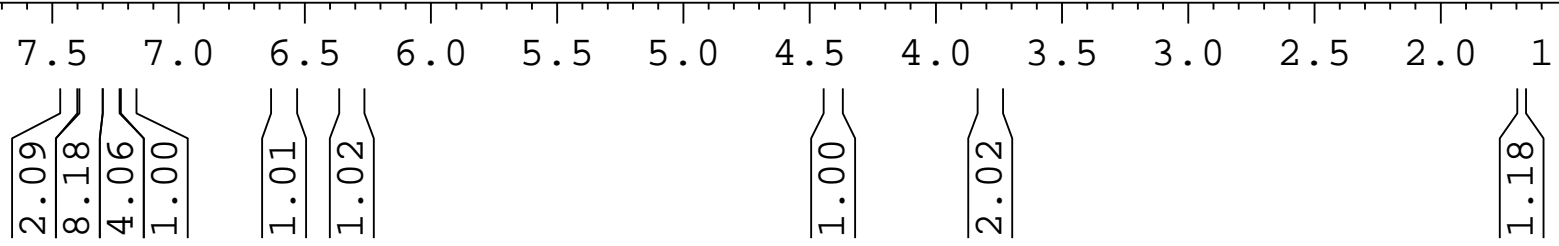


ம

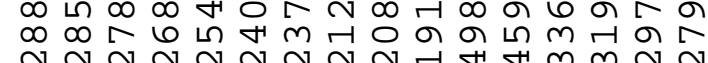

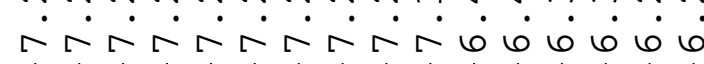

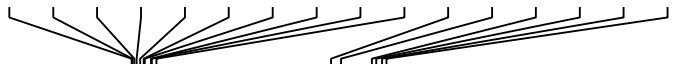

๑ோ

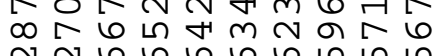

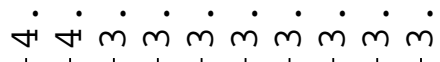
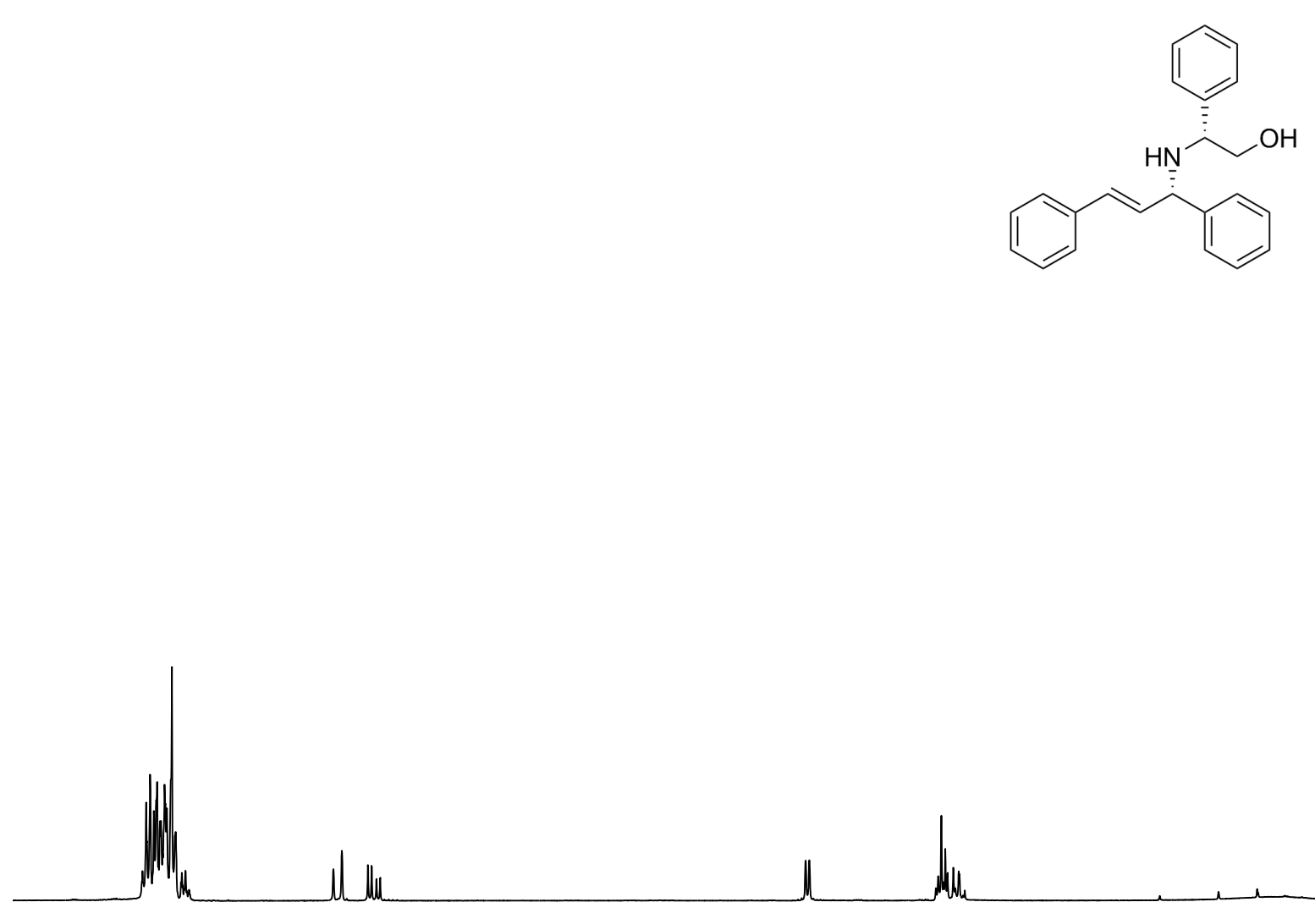

NAME

EXPNO

PROCNO

Date
Time

INSTRUM

PROBHD

PULPROG

TD

SOLVENT

NS

SWH

FIDRES

$\mathrm{RG}$

DW

DE

TE

TDO

$=====$

NUC1

P1

PL1W

SF01

SI

SF

SSB

LB

$\mathrm{GB}$

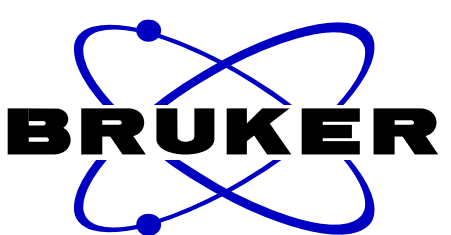

LSS180929-4-2

20181002 14.09 $\mathrm{mm}$ РABBO BBzg30 65536 $\mathrm{CDCl3}$

$16163.793 \mathrm{~Hz}$ $0.246640 \mathrm{~Hz}$ $2.0272970 \mathrm{sec}$ 203

30.933 usec 6.50 usec $296.1 \mathrm{~K}$

$1.00000000 \mathrm{sec}$

$=$ CHANNEL $f$

\section{$=======$} 15.50 usec $1.00 \mathrm{~dB}$ $13.18669796 \mathrm{~W}$ $400.1728012 \mathrm{MHz}$ 32768 $400.1700050 \mathrm{MHz}$

EM
0
$0.30 \mathrm{~Hz}$
0
0

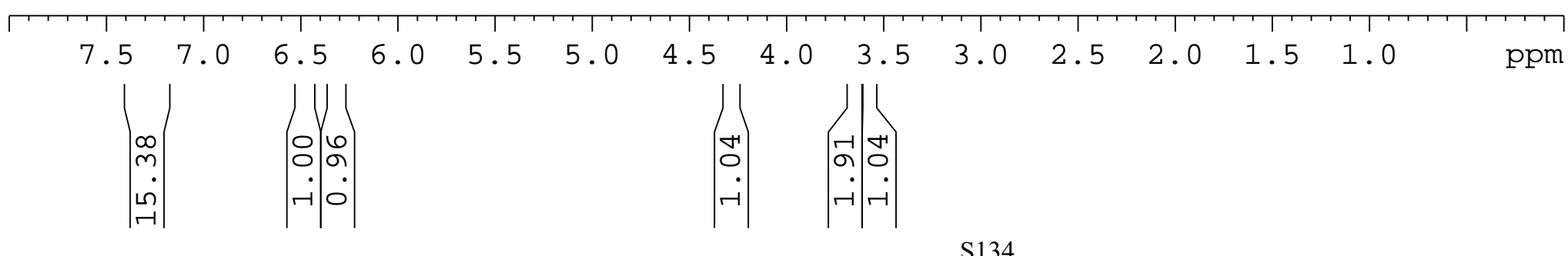




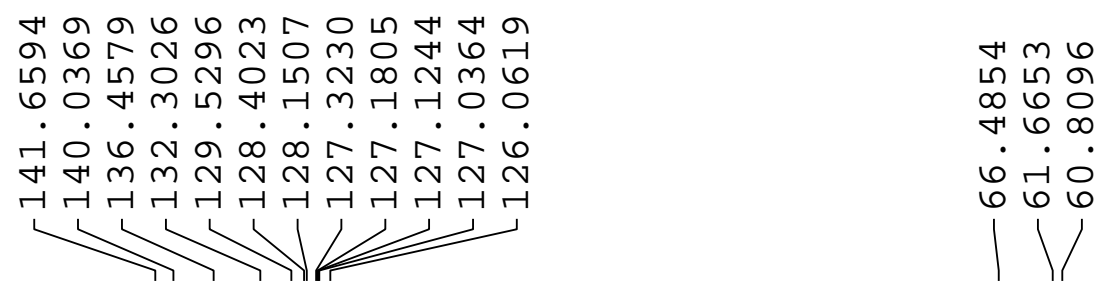

귬용

$0 \infty$

060

AME

EXPNO

PROCNO

Date

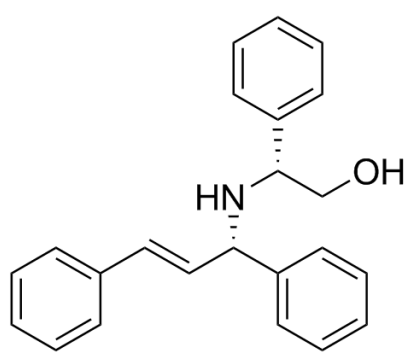

INSTRUM

INSTRU

PROBHD

PULPROG

SOLVENT

NS

DS

SWH

FIDRES

AQ

RG

DW

DE

D1

sione (x)

LTY180921-C13

2018101

10.55

$5 \mathrm{~mm}$ spect

zgpg 30

65536

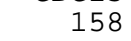

$71428.570 \mathrm{~Hz}$

$1.089913 \mathrm{~Hz}$

$0.4588020 \mathrm{sec}$ 203

7.000 usec

6.50 usec

$296.3 \mathrm{~K}$

$2.00000000 \mathrm{sec}$

$0.03000000 \mathrm{sec}$

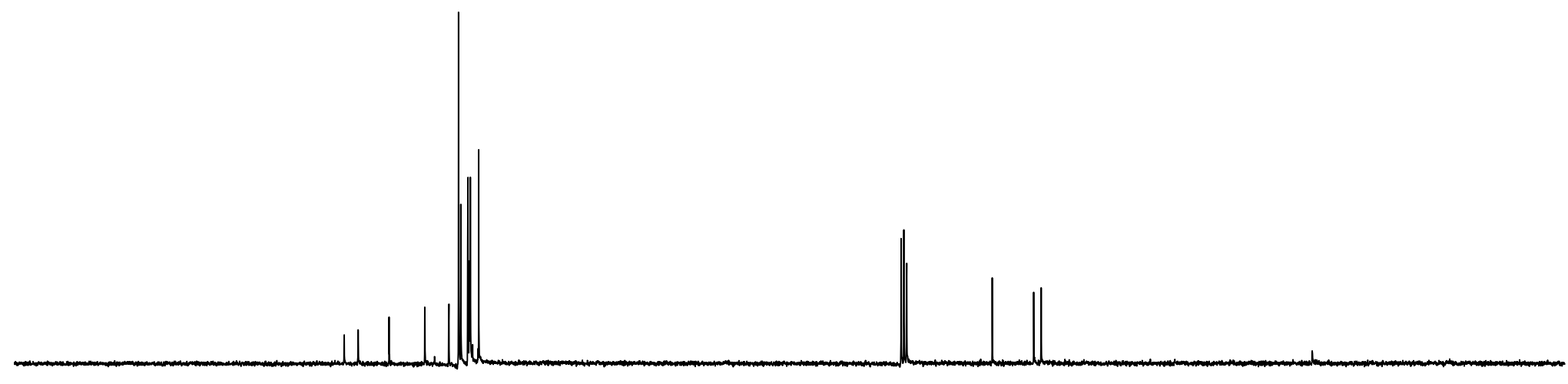

$=======$ CHANNEL NUC1

PL1

PL1W

=====-== $13 \mathrm{C}$

$57.32743073 \mathrm{~W}$

CPDPRG2

NUC2

$100.6228270 \mathrm{MHz}$

NUC2

PL12

PL13

PL2W

PL12W

PL13W

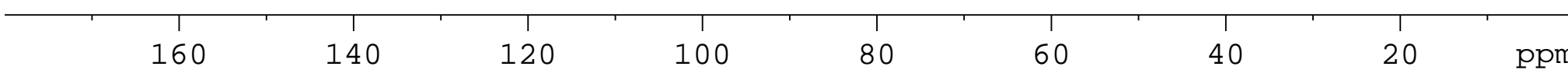

140

120

100

80

60

ppm

SSB

GB

CHANNEL f2 ========
waltz16
$1 \mathrm{H}$
$80.00 \mathrm{usec}$
$-1.00 \mathrm{~dB}$
$13.26 \mathrm{~dB}$
$14.46 \mathrm{~dB}$
$13.18669796 \mathrm{~W}$
$0.49446553 \mathrm{~W}$
$0.37509048 \mathrm{~W}$
$400.1716007 \mathrm{MHz}$
$32768 \mathrm{MH}$
$10 \odot .6228648 \mathrm{MHz}$
$\mathrm{EM}$
$\odot$
$1.0 \odot \mathrm{Hz}$
$\odot$
1.40


N

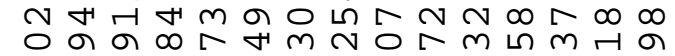

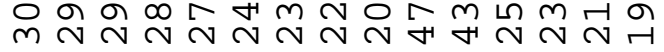
ن 3

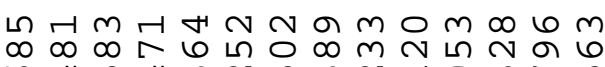
0 \& N $\odot \odot \odot \odot \wedge N \wedge N \circlearrowleft$

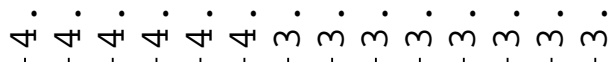
-

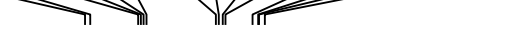

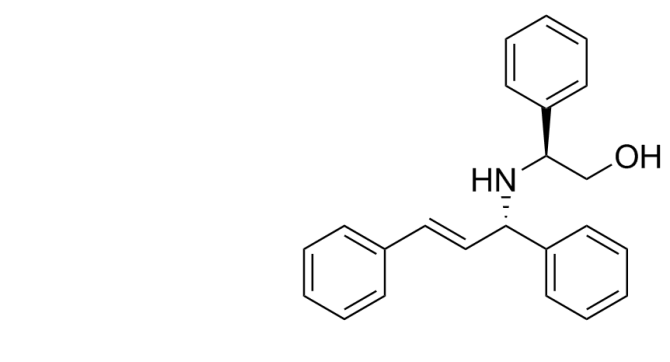

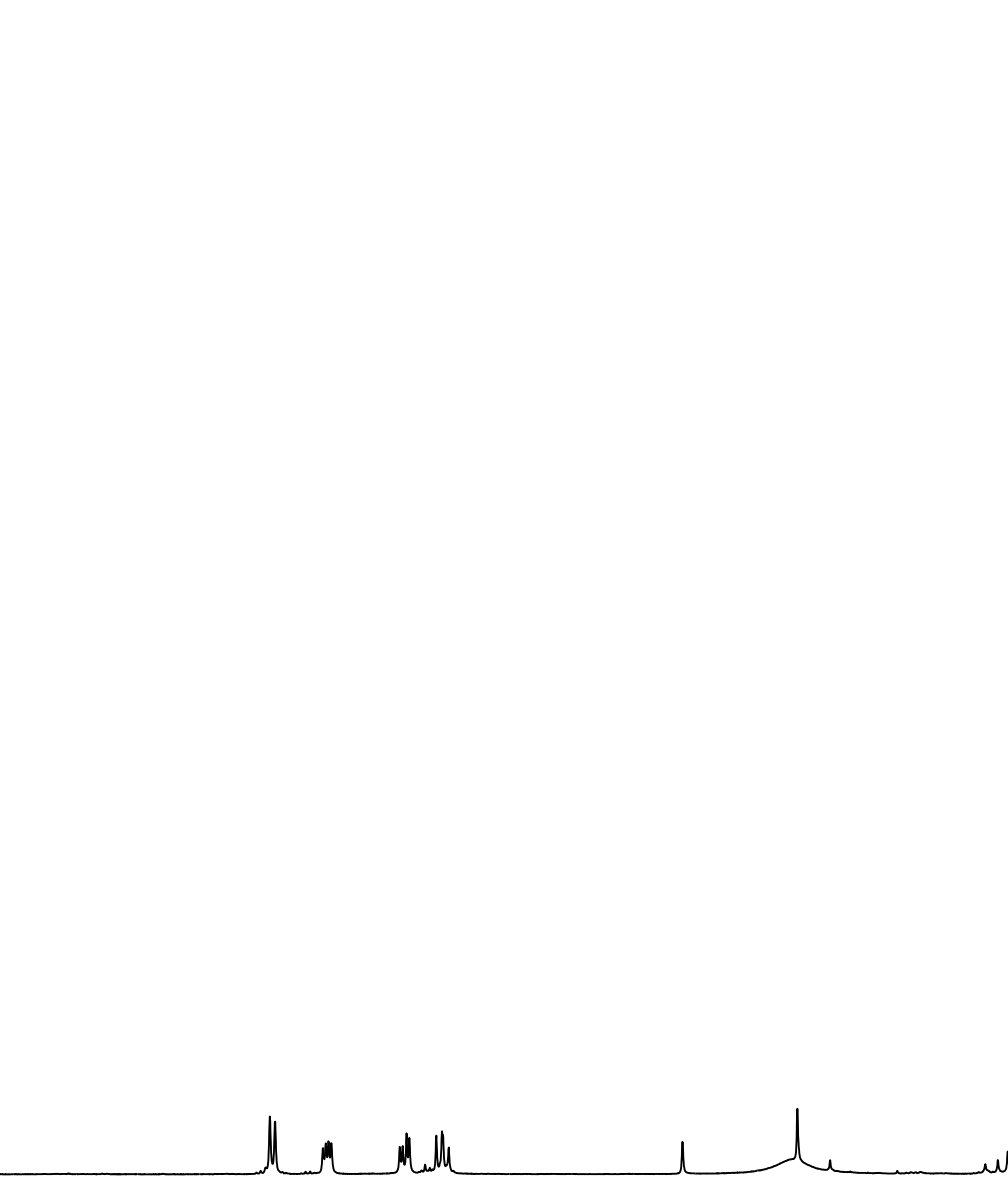

NAME

EXPNO

PROCNO

Date

INSTRUM

PROBHD

PULPROG

TD

SOLVENT

NS

DS

SWH

AQ

RG

DW

DE

TE

TD

\section{$====$}

NUC1

PL1

PL1W

SF01

SI

SF
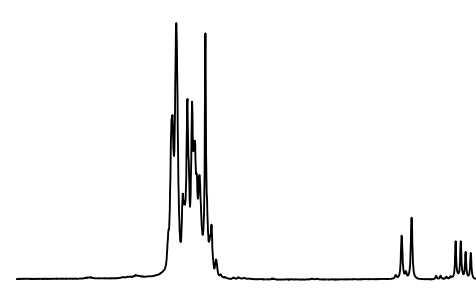

M llu

, 5

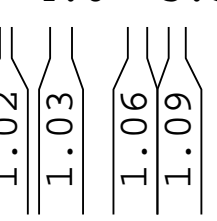

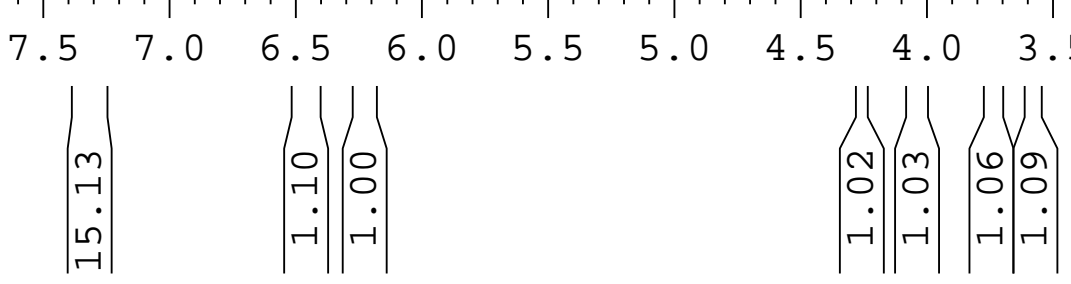

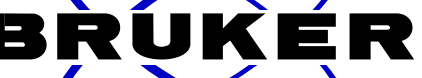

$C<1$

LSS181009

15.12

$5 \mathrm{~mm} P A B B O$ ect

zg30

CDCl3

$16163.793 \mathrm{~Hz}$

$0.246640 \mathrm{~Hz}$

$2.0272970 \mathrm{sec}$

203

30.933 usec

6.50 usec

$295.4 \mathrm{~K}$

$1.00000000 \mathrm{sec}$

$=$ CHANNEL $f$

$=======$ $5.1 \mathrm{H}$ use 15.50 usec $13.18669796 \mathrm{~W}$ $400.1728012 \mathrm{MHz}$ 32768 $400.1700070 \mathrm{MHz}$

$\mathrm{EM}$
0
$30 \mathrm{~Hz}$
0
0




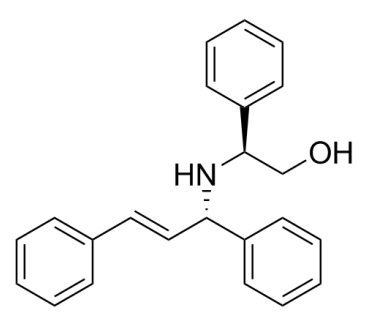

NAME

EXPNO

PROCNO

Date

INIme

PROBHD

PULPROG

TD

SOLVENT

NS
DS
SWH

FIDRES

$A Q$

RG

DW

DE

D1

D11
TD 9

\section{(1)}

NUC1

P1

PL1

SF01

$======$

CPDPRG2

NUC2

PCPD

PL2

PL13

PL $2 W$

PL2W

PL13W

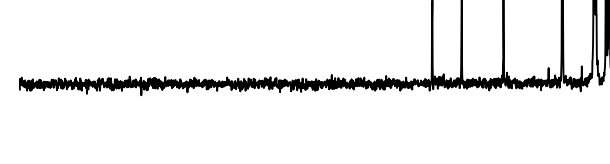

160

140

120

100

80

60

40

20

ppm

SF

SSB

LB

PC
?

LSS181009-C

20181012 15.22

$5 \mathrm{~mm}$ РАВBO BB-

zgpg30

65536

137

4
$71428.570 \mathrm{~Hz}$

1. $089913 \mathrm{~Hz}$

$0.4588020 \mathrm{sec}$

203

7.000 usec

6.50 usec

$296.5 \mathrm{~K}$

$2.00000000 \mathrm{sec}$

$0.03000000 \mathrm{~s}$

$==$ CHANNEL $\mathrm{f}$

\section{$=======$} $13 \mathrm{C}$

8.50 use
$2.00 \mathrm{~dB}$

$57.32743073 \mathrm{~W}$

$100.6228270 \mathrm{MHz}$

CHANNEL f2 ======== waltz16

80.00 usec

80.00 usec

$-1.00 \mathrm{~dB}$

$13.26 \mathrm{~dB}$
$14.46 \mathrm{~dB}$

$13.18669796 \mathrm{~W}$

$0.49446553 \mathrm{~W}$

$0.37509048 \mathrm{~W}$

$40 \odot .1716007 \mathrm{MHz}$

32768

32768
100.6228294
$M H z$

EM

1. $\stackrel{\ominus}{\circ} \mathrm{Hz}$

1.00
0 


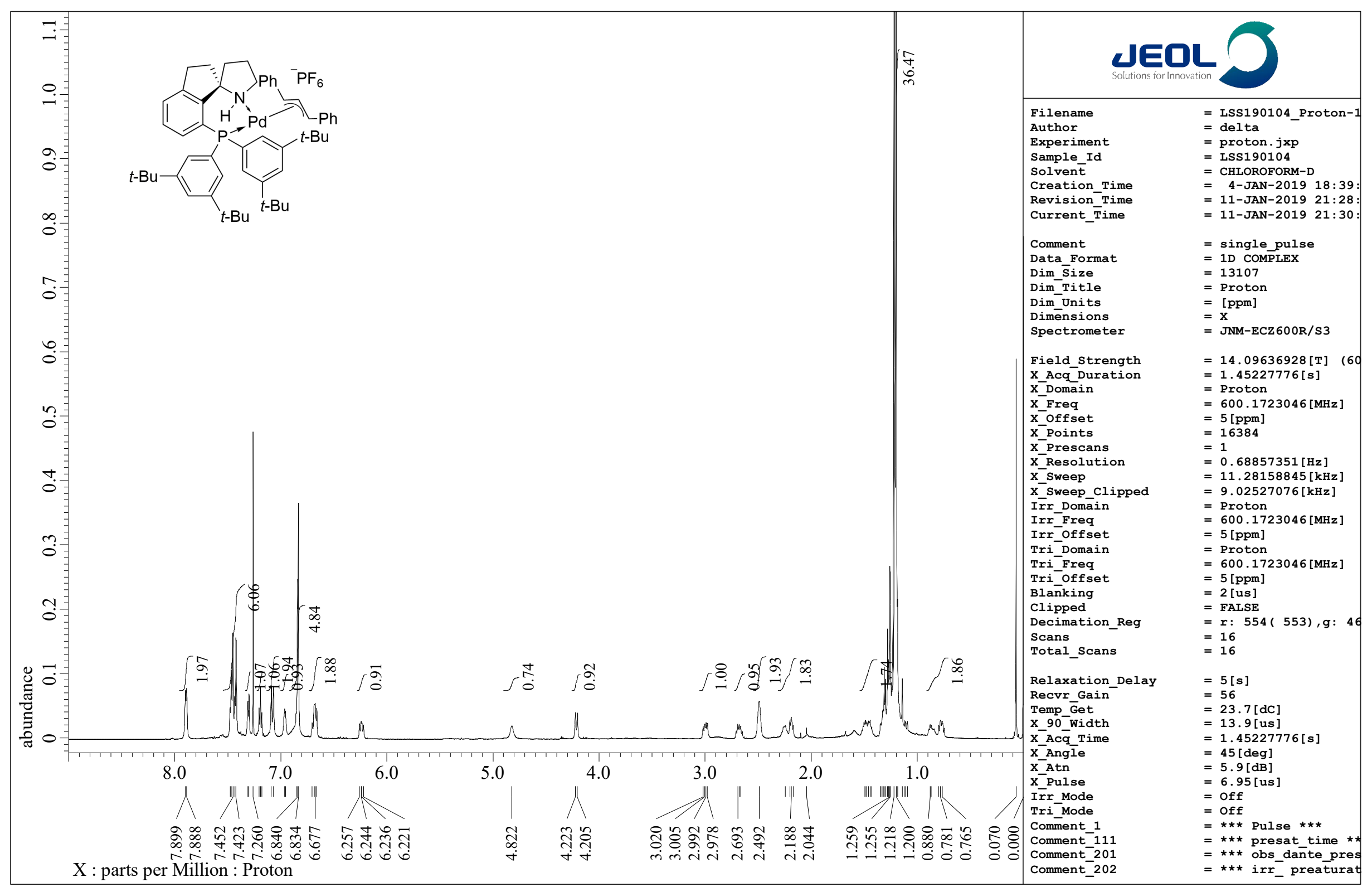




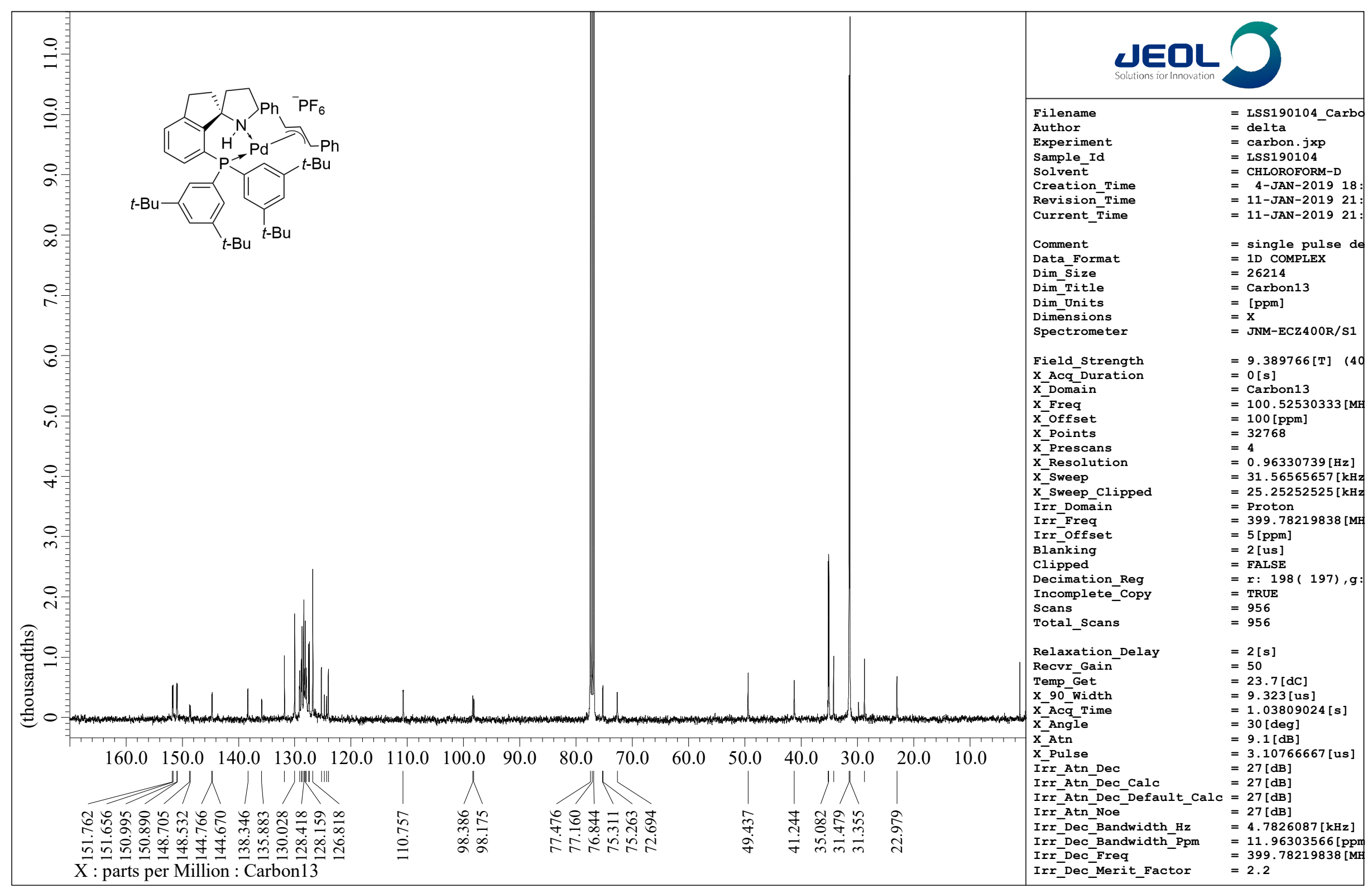




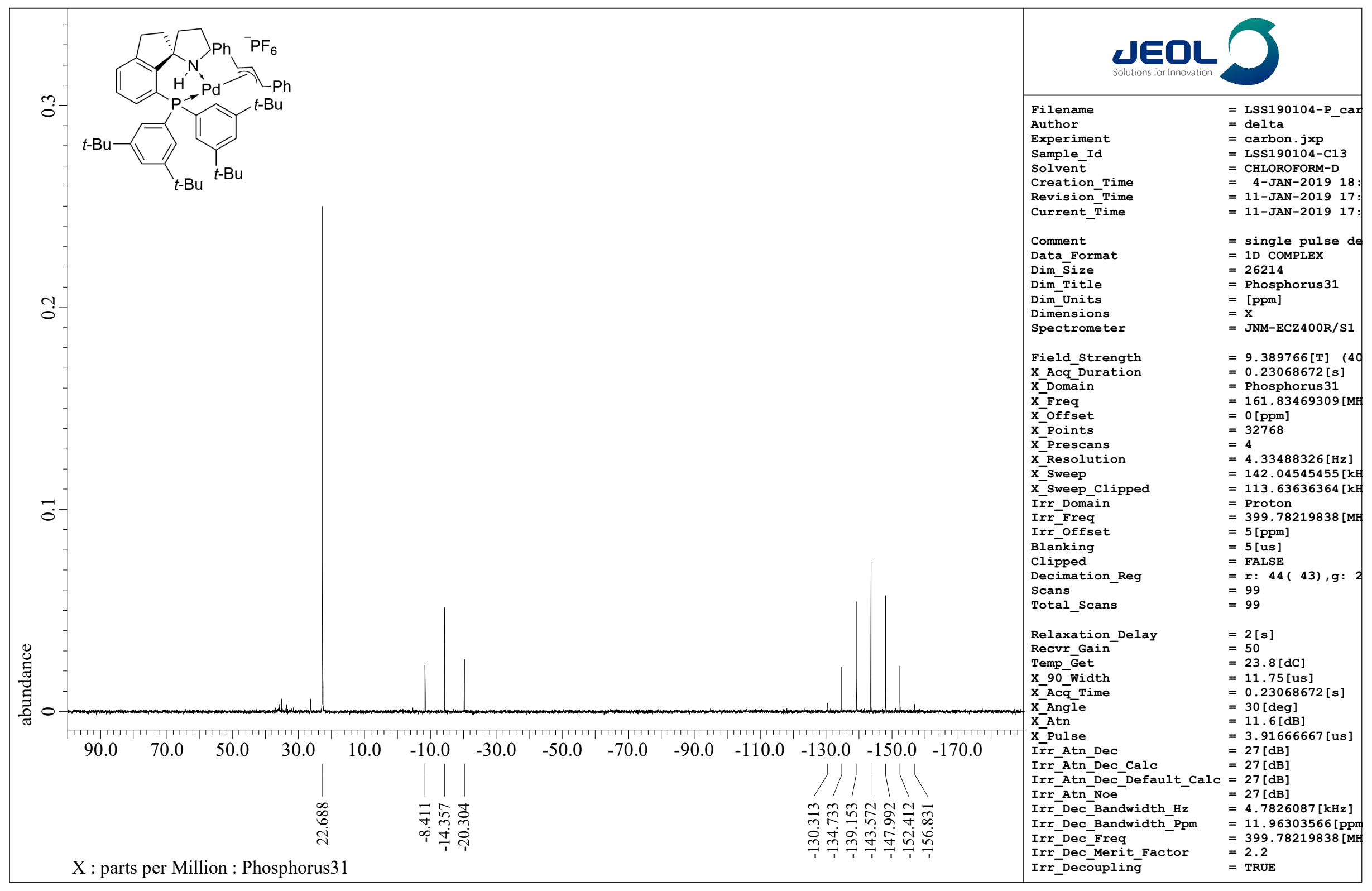




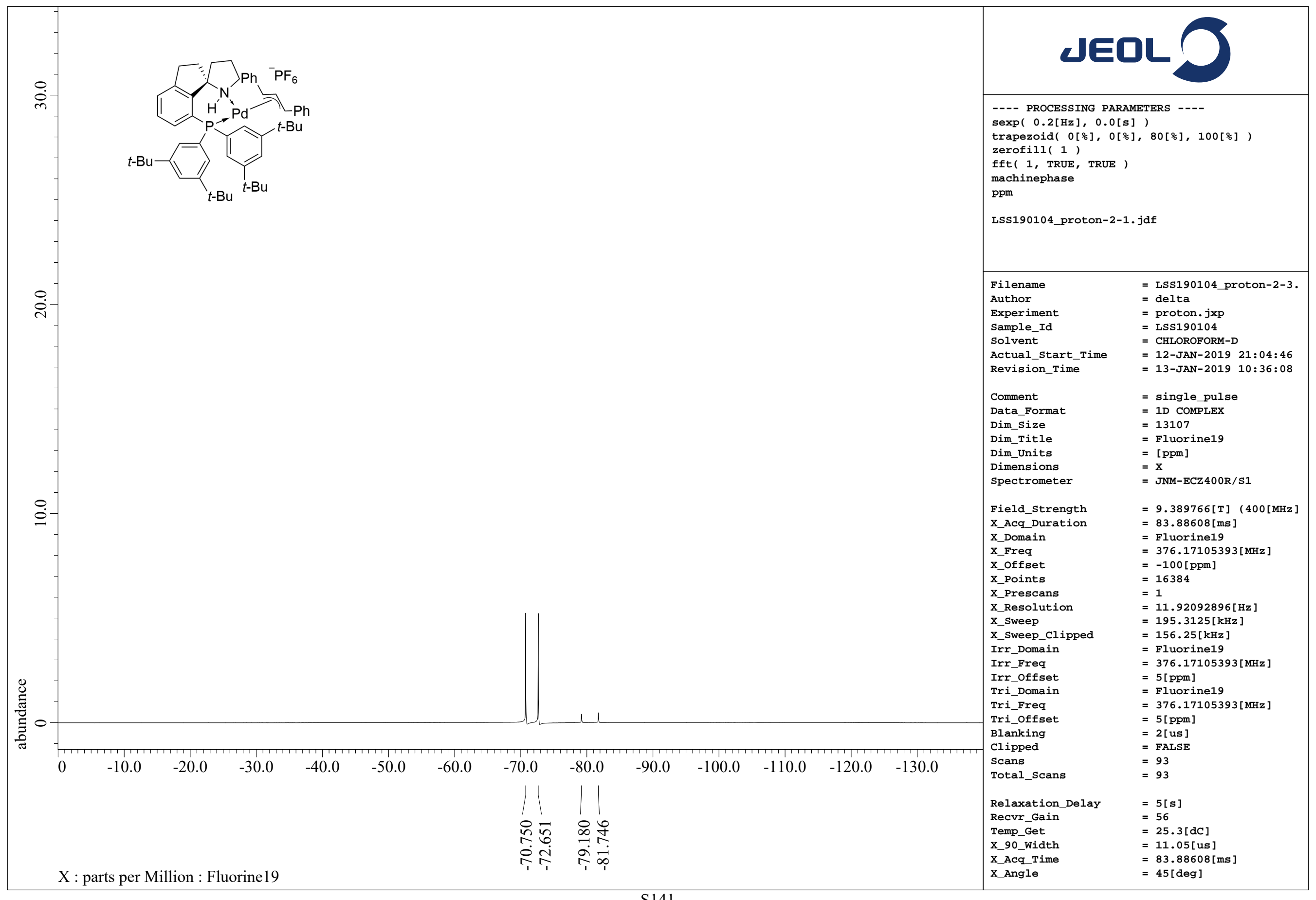




\section{==== Analysis Report ====}

Acquired by

Sample Name

Sample ID

Vail \#

Injection Volume

Data File Name

Method File Name

Batch File Name

Report File Name

Data Acquired

Data Processed
E:Idatal901IFLFIPJ160819 AD 95 5.Icd

: Admin

: PJ160819 AD 955

: $1 \mathrm{uL}$

PJ160819 AD 95 5.Icd

: method1.Icm

Default.Icr

: 2016/9/24 11:00:04

: 2016/9/24 11:26:24

\section{$<$ Chromatogram >}

$\mathrm{mV}$

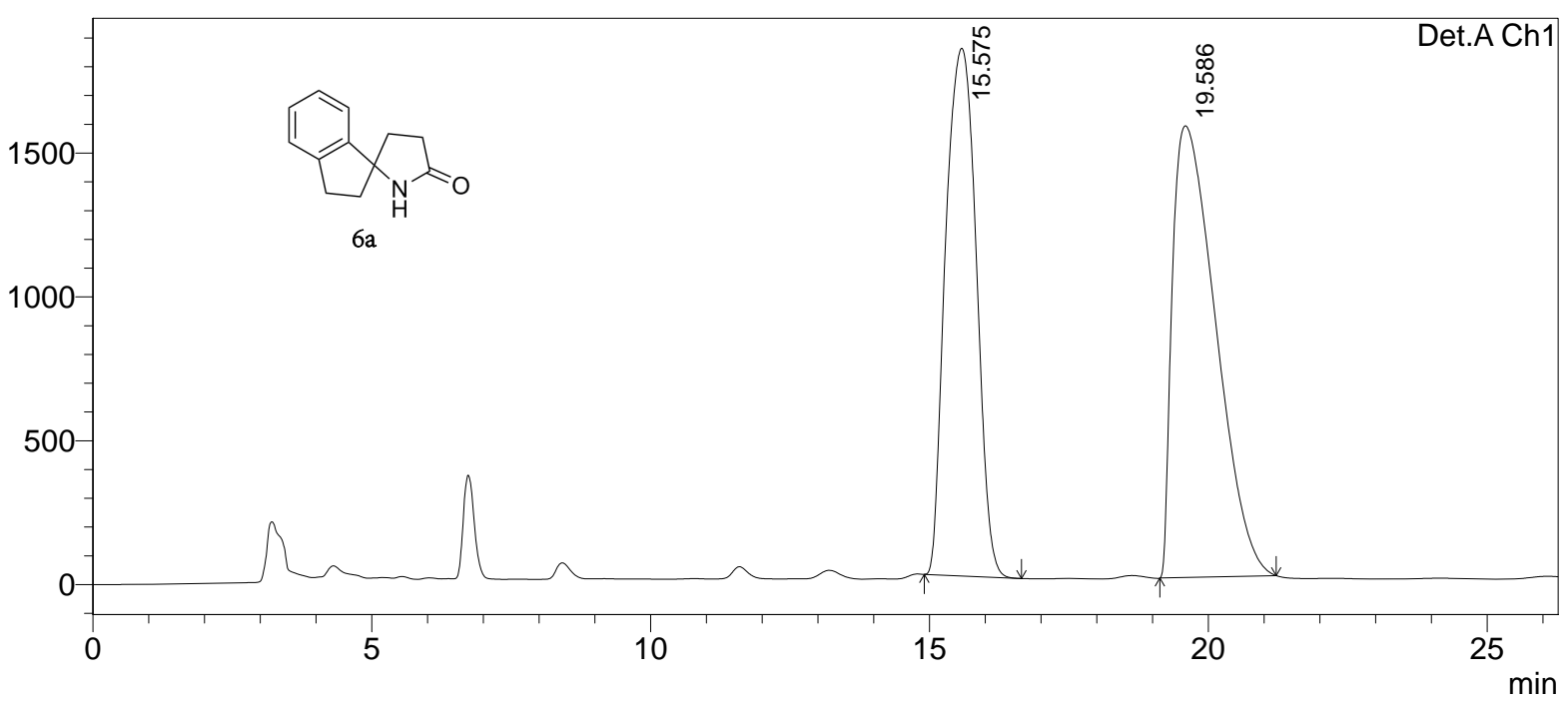

1 Det.A Ch1/220nm

PeakTable

Detector A Ch1 220nm

\begin{tabular}{|r|r|r|r|r|r|}
\hline \multicolumn{1}{|c|}{ Peak\# } & \multicolumn{1}{|c|}{ Ret. Time } & \multicolumn{1}{c|}{ Area } & Height & \multicolumn{1}{c|}{ Area \% } & \multicolumn{1}{c|}{ Height \% } \\
\hline 1 & 15.575 & 70244085 & 1834930 & 45.346 & 53.880 \\
\hline 2 & 19.586 & 84663731 & 1570627 & 54.654 & 46.120 \\
\hline Total & & 154907816 & 3405557 & 100.000 & 100.000 \\
\hline
\end{tabular}




\section{=ニニ= Analysis Report $====$}

$\begin{array}{ll} & \\ \text { Acquired by } & \text { : Admin } \\ \text { Sample Name } & : \text { CWG160128 } \\ \text { Sample ID } & : \\ \text { Vail \# } & : \text { 1 uL } \\ \text { Injection Volume } & : \text { CWG160129.Icd } \\ \text { Data File Name } & : \text { method1.Icm } \\ \text { Method File Name } & : \\ \text { Batch File Name } & \text { : Default.Icr } \\ \text { Report File Name } & : \text { 2016/8/29 15:15:38 } \\ \text { Data Acquired } & : \text { 2016/8/29 15:40:02 } \\ \text { Data Processed } & \end{array}$

\section{$<$ Chromatogram>}

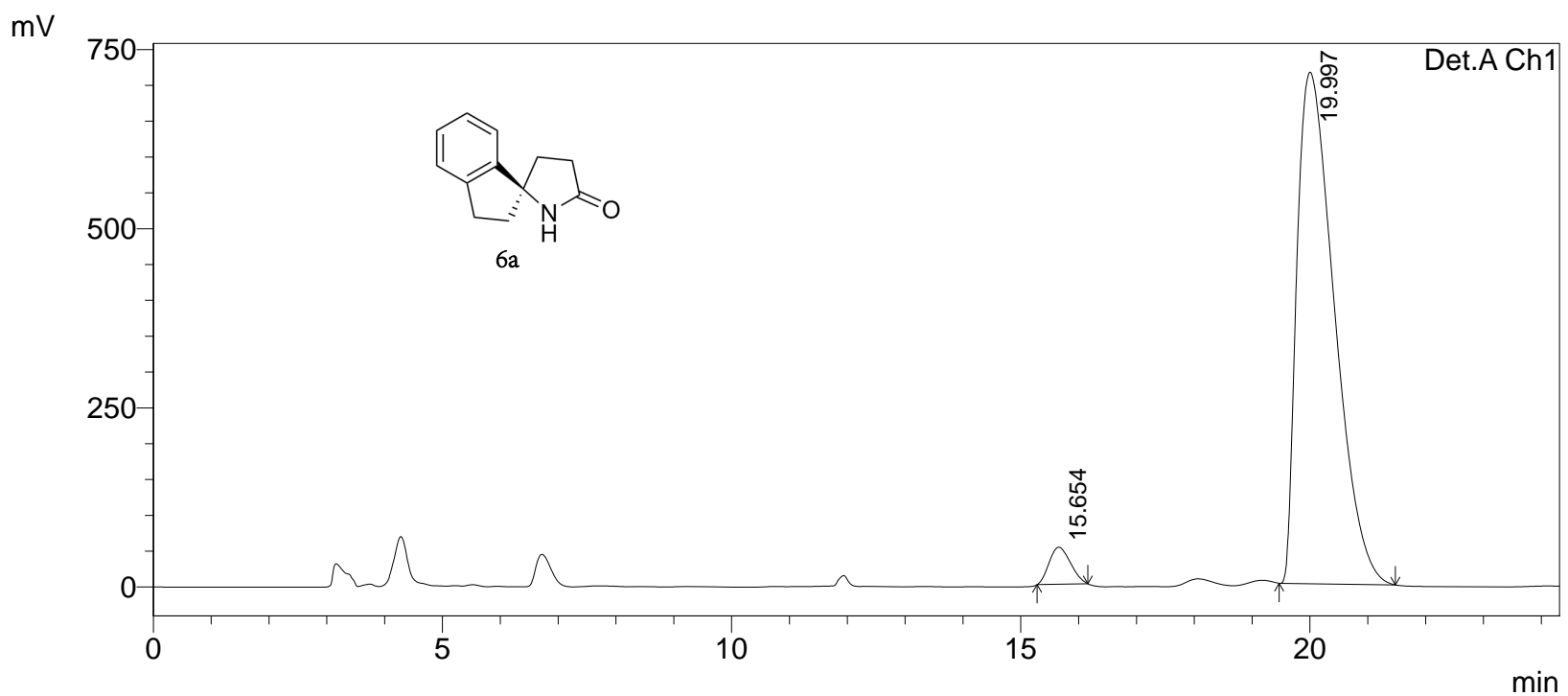

1 Det.A Ch1/220nm

PeakTable

Detector A Ch1 220nm

\begin{tabular}{|r|r|r|r|r|r|}
\hline Peak\# & Ret. Time & \multicolumn{1}{|c|}{ Area } & Height & \multicolumn{1}{|c|}{ Area \% } & \multicolumn{1}{c|}{ Height \% } \\
\hline 1 & 15.654 & 1322317 & 51679 & 3.959 & 6.751 \\
\hline 2 & 19.997 & 32082006 & 713807 & 96.041 & 93.249 \\
\hline Total & & 33404323 & 765486 & 100.000 & 100.000 \\
\hline
\end{tabular}




\section{==== Analysis Report ====}

Acquired by

Sample Name

Sample ID

Vail \#

Injection Volume

Data File Name

Method File Name

Batch File Name

Report File Name

Data Acquired

Data Processed
E:Idatal901IFLFIZJX170810 OD 90 10.Icd

: Admin

: ZJX170810-1 OD 9010

$: 1 \mathrm{uL}$

ZJX170810 OD 90 10.Icd

: method1.Icm

: Default.Icr

2017/8/11 14:45:52

: 2017/8/11 15:09:29

\section{$<$ Chromatogram >}

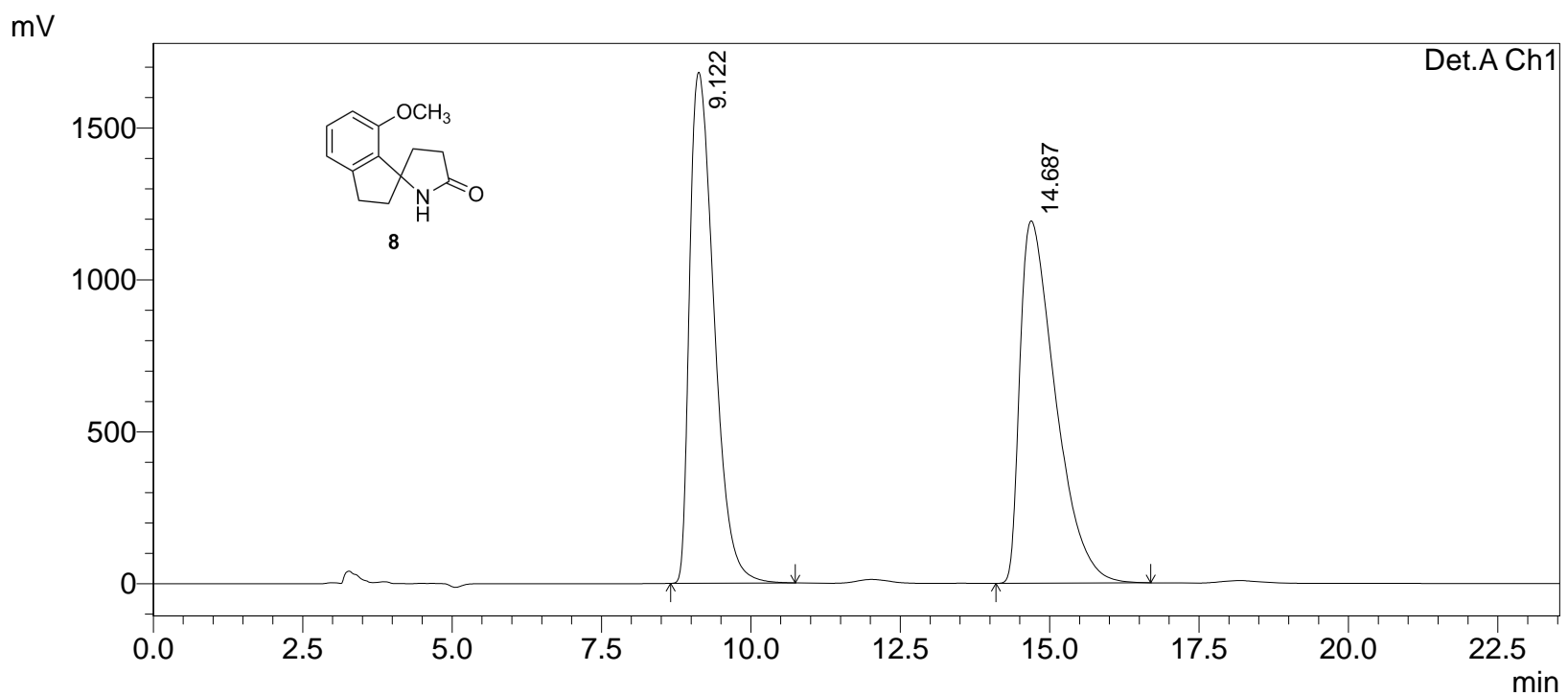

1 Det.A Ch1/220nm

Detector A Ch1 220nm

PeakTable

\begin{tabular}{|r|r|r|r|r|r|}
\hline \multicolumn{1}{|c|}{ Peak\# } & Ret. Time & \multicolumn{1}{|c|}{ Area } & Height & Area \% & \multicolumn{1}{|c|}{ Height \% } \\
\hline 1 & 9.122 & 47871900 & 1681961 & 49.296 & 58.506 \\
\hline 2 & 14.687 & 49239549 & 1192897 & 50.704 & 41.494 \\
\hline Total & & 97111449 & 2874858 & 100.000 & 100.000 \\
\hline
\end{tabular}




\section{==== Analysis Report ====}

Acquired by

Sample Name

Sample ID

Vail \#

Injection Volume

Data File Name

Method File Name

Batch File Name

Report File Name

Data Acquired

Data Processed
E:Idatal901IFLFIZJX170726-1 OD 90 10.Icd

: Admin

: ZJX170726-1 OD 9010

$: 1 \mathrm{uL}$

: ZJX170726-1 OD 90 10.Icd

: method1.Icm

: Default.Icr

:2017/8/11 15:10:06

: 2017/8/11 15:30:48

\section{$<$ Chromatogram >}

$\mathrm{mV}$

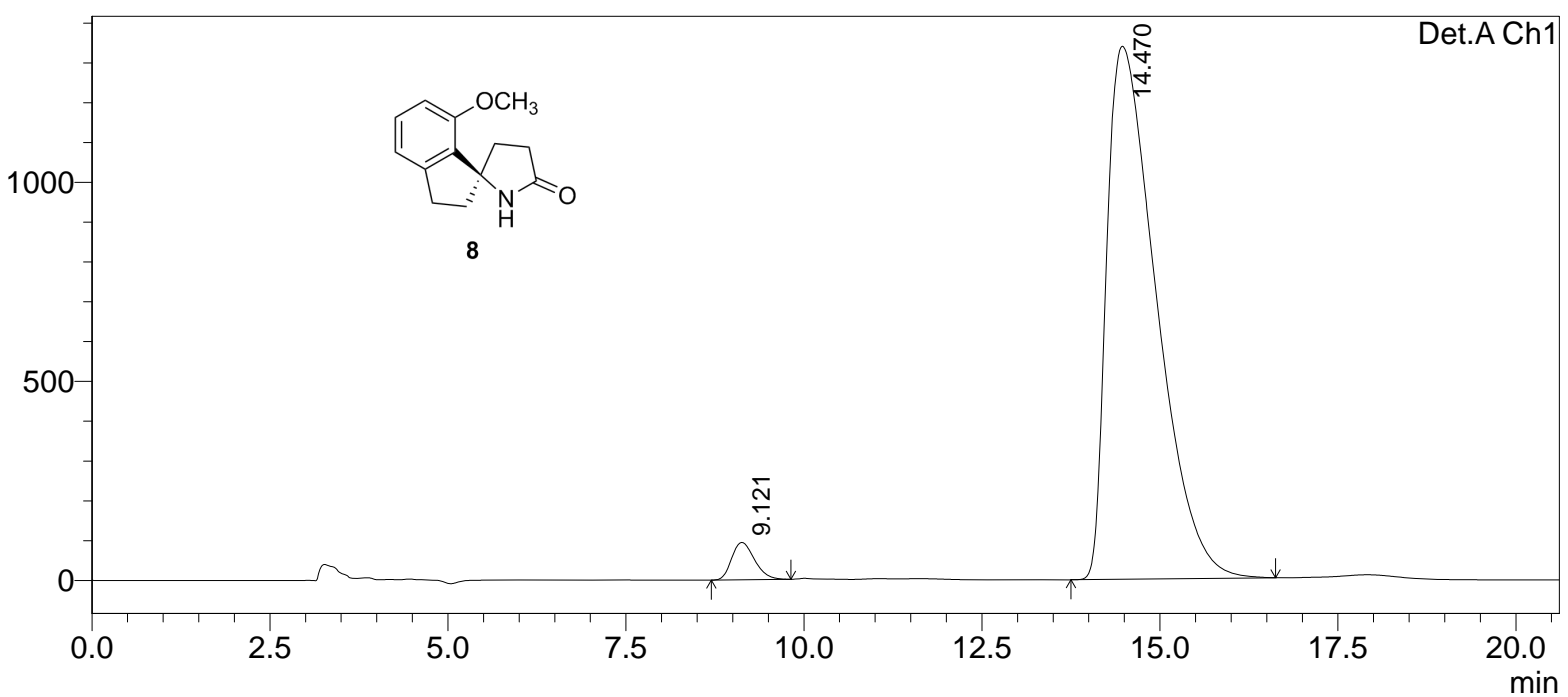

1 Det.A Ch1/220nm

PeakTable

Detector A Ch1 220nm

\begin{tabular}{|r|r|r|r|r|r|}
\hline Peak\# & Ret. Time & \multicolumn{1}{|c|}{ Area } & Height & \multicolumn{1}{|c|}{ Area \% } & \multicolumn{1}{c|}{ Height \% } \\
\hline 1 & 9.121 & 2093054 & 93971 & 3.130 & 6.557 \\
\hline 2 & 14.470 & 64784122 & 1339148 & 96.870 & 93.443 \\
\hline Total & & 66877176 & 1433118 & 100.000 & 100.000 \\
\hline
\end{tabular}




\section{==== Analysis Report ====}

Acquired by

Sample Name

Sample ID

Vail \#

Injection Volume

Data File Name

Method File Name

Batch File Name

Report File Name

Data Acquired

Data Processed
E:Idatal901IFLFIFLF170904-2 OD 90 10.Icd

: Admin

FLF170904-2 OD 9010

: Default.Icr

: 2017/9/6 15:41:15

: 2017/9/6 16:06:08

\section{$<$ Chromatogram>}

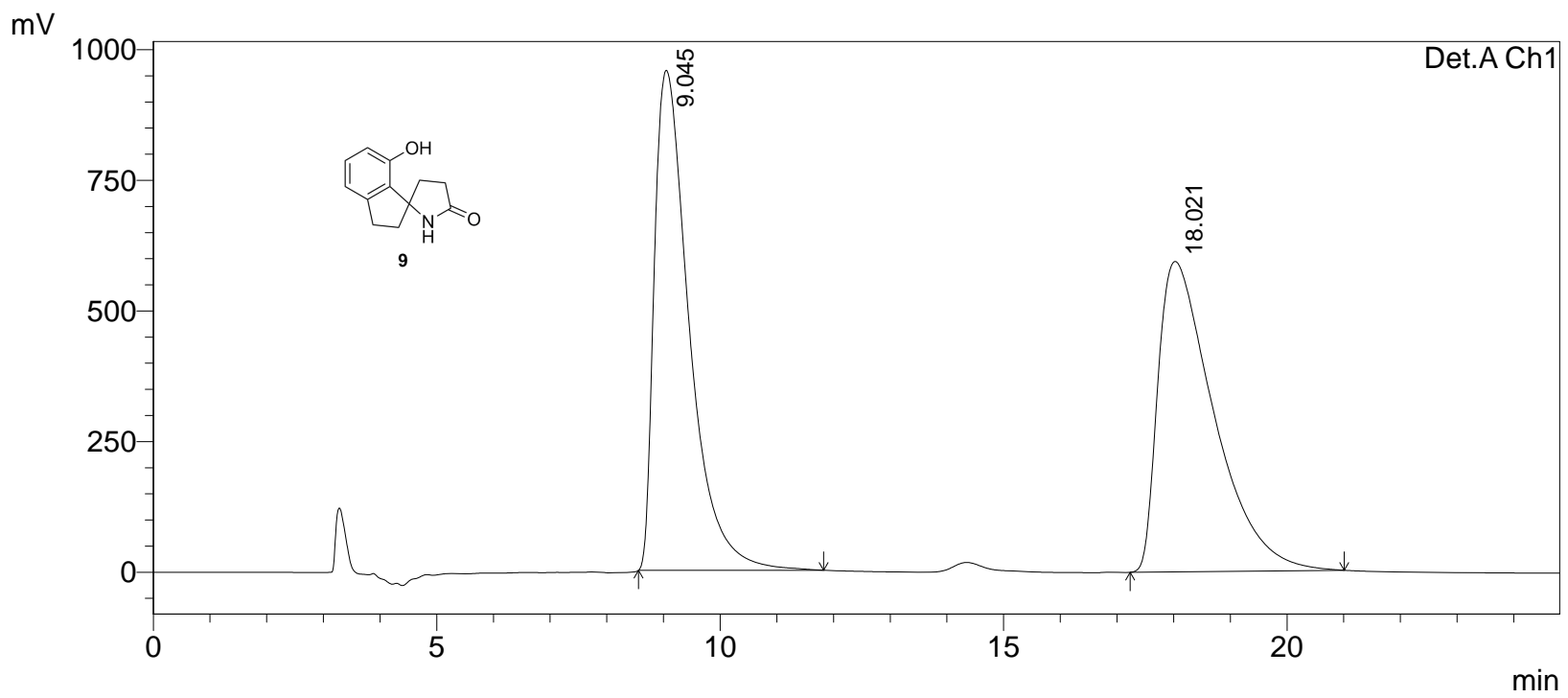

1Det.A Ch1/220nm

PeakTable

Detector A Ch1 220nm

\begin{tabular}{|r|r|r|r|r|r|}
\hline \multicolumn{1}{|c|}{ Peak\# } & Ret. Time & Area & \multicolumn{1}{|c|}{ Height } & Area \% & \multicolumn{1}{c|}{ Height \% } \\
\hline 1 & 9.045 & 40974975 & 957012 & 50.117 & 61.702 \\
\hline 2 & 18.021 & 40782981 & 594002 & 49.883 & 38.298 \\
\hline Total & & 81757956 & 1551014 & 100.000 & 100.000 \\
\hline
\end{tabular}




\section{==== Analysis Report ====}

Acquired by

Sample Name

Sample ID

Vail \#

Injection Volume

Data File Name

Method File Name

Batch File Name

Report File Name

Data Acquired

Data Processed
E:Idatal901IFLFILSS170726-1 OD 90 10.Icd

: Admin

: LSS170726-1 OD 9010

: $1 \mathrm{uL}$

: LSS170726-1 OD 90 10.Icd

: method1.Icm

Default.Icr

2017/8/4 17:12:42

: 2018/5/25 9:56:40

\section{$<$ Chromatogram >}

$\mathrm{mV}$

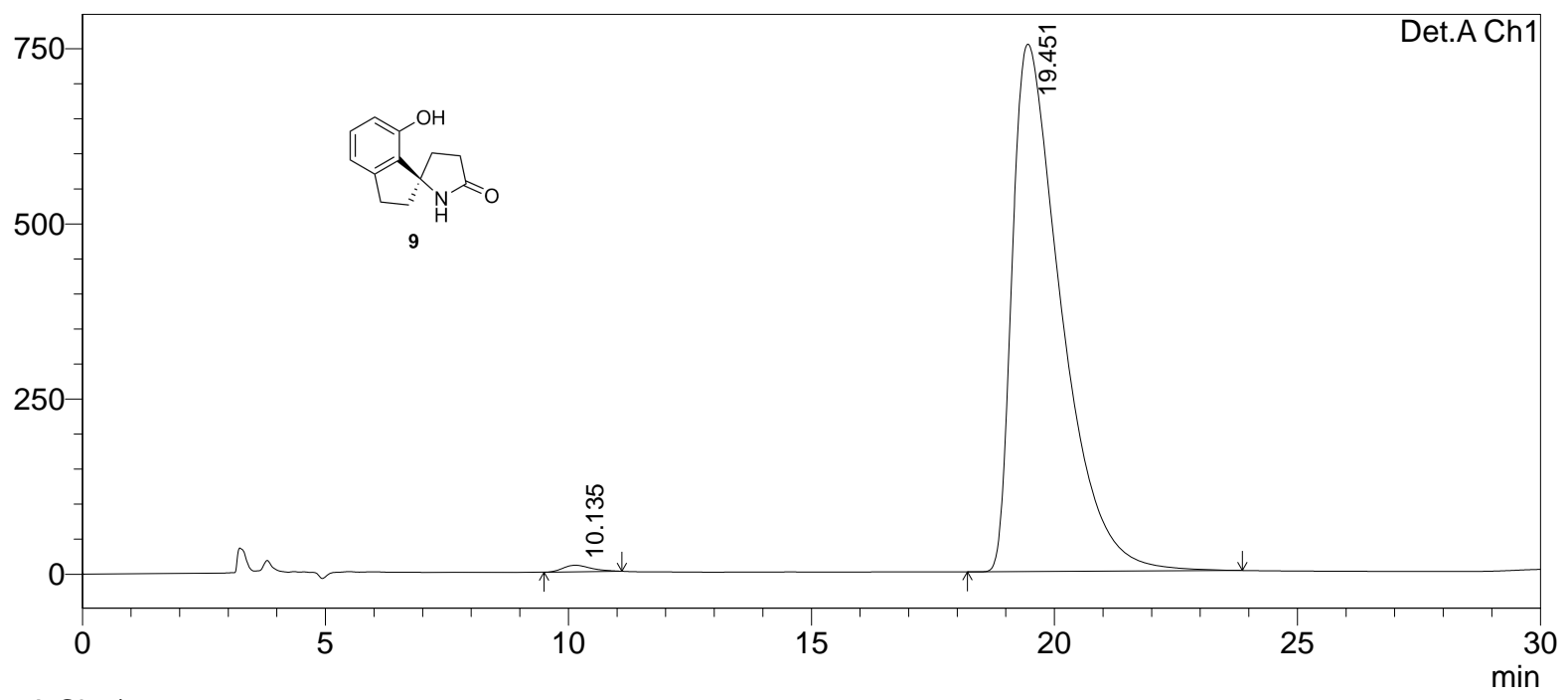

1 Det.A Ch1/220nm

PeakTable

Detector A Ch1 220nm

\begin{tabular}{|r|r|r|r|r|r|}
\hline \multicolumn{1}{|c|}{ Peak\# } & Ret. Time & \multicolumn{1}{c|}{ Area } & \multicolumn{1}{c|}{ Height } & \multicolumn{1}{c|}{ Area \% } & \multicolumn{1}{c|}{ Height \% } \\
\hline 1 & 10.135 & 382201 & 9438 & 0.720 & 1.238 \\
\hline 2 & 19.451 & 52695670 & 752864 & 99.280 & 98.762 \\
\hline Total & & 53077871 & 762301 & 100.000 & 100.000 \\
\hline
\end{tabular}




\section{==== Analysis Report ====}

Acquired by Sample Name Sample ID

Vail \#

Injection Volume Data File Name

Method File Name

Batch File Name

Report File Name

Data Acquired

Data Processed
E:Idatal901IFLFIFLF180316(OD 10\% 210 1)1.Icd

: Admin

: FLF180316(OD 10\% 210 1)

$: 1 \mathrm{uL}$

: FLF180316(OD 10\% 210 1)1.Icd

: method1.Icm

: Default.Icr

2018/3/23 15:00:07

: 2018/3/23 15:52:59

\section{$<$ Chromatogram>}

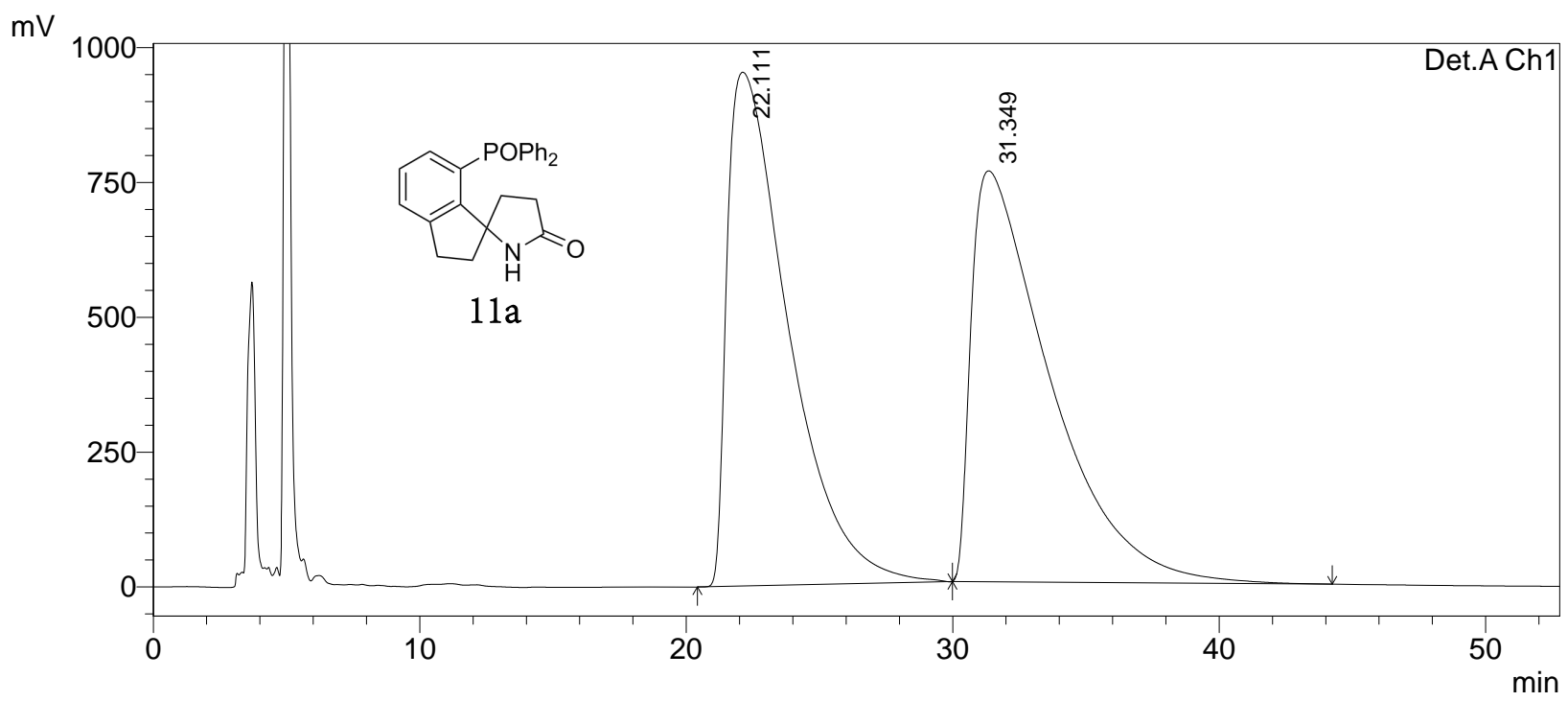

1 Det.A Ch1/210nm

Detector A Ch1 210nm

PeakTable

\begin{tabular}{|r|r|r|r|r|r|}
\hline Peak\# & Ret. Time & \multicolumn{1}{|c|}{ Area } & Height & \multicolumn{1}{|c|}{ Area \% } & \multicolumn{1}{c|}{ Height \% } \\
\hline 1 & 22.111 & 152213359 & 952802 & 49.313 & 55.572 \\
\hline 2 & 31.349 & 156453699 & 761720 & 50.687 & 44.428 \\
\hline Total & & 308667058 & 1714522 & 100.000 & 100.000 \\
\hline
\end{tabular}




\section{=ニニ= Analysis Report $====$}

Acquired by Sample Name Sample ID

Vail \#

Injection Volume

Data File Name

Method File Name

Batch File Name

Report File Name

Data Acquired

Data Processed

\section{E:Idatal901IFLFIFLF180301-1(OD 10\% 210 1)1.Icd}

: Admin

FLF180301-1(OD 10\% 210 1)

$: 1 \mathrm{uL}$

: FLF180301-1(OD 10\% 210 1)1.Icd

: method1.Icm

Default.Icr

2018/3/23 15:54:37

: 2018/3/23 16:37:01

\section{$<$ Chromatogram >}

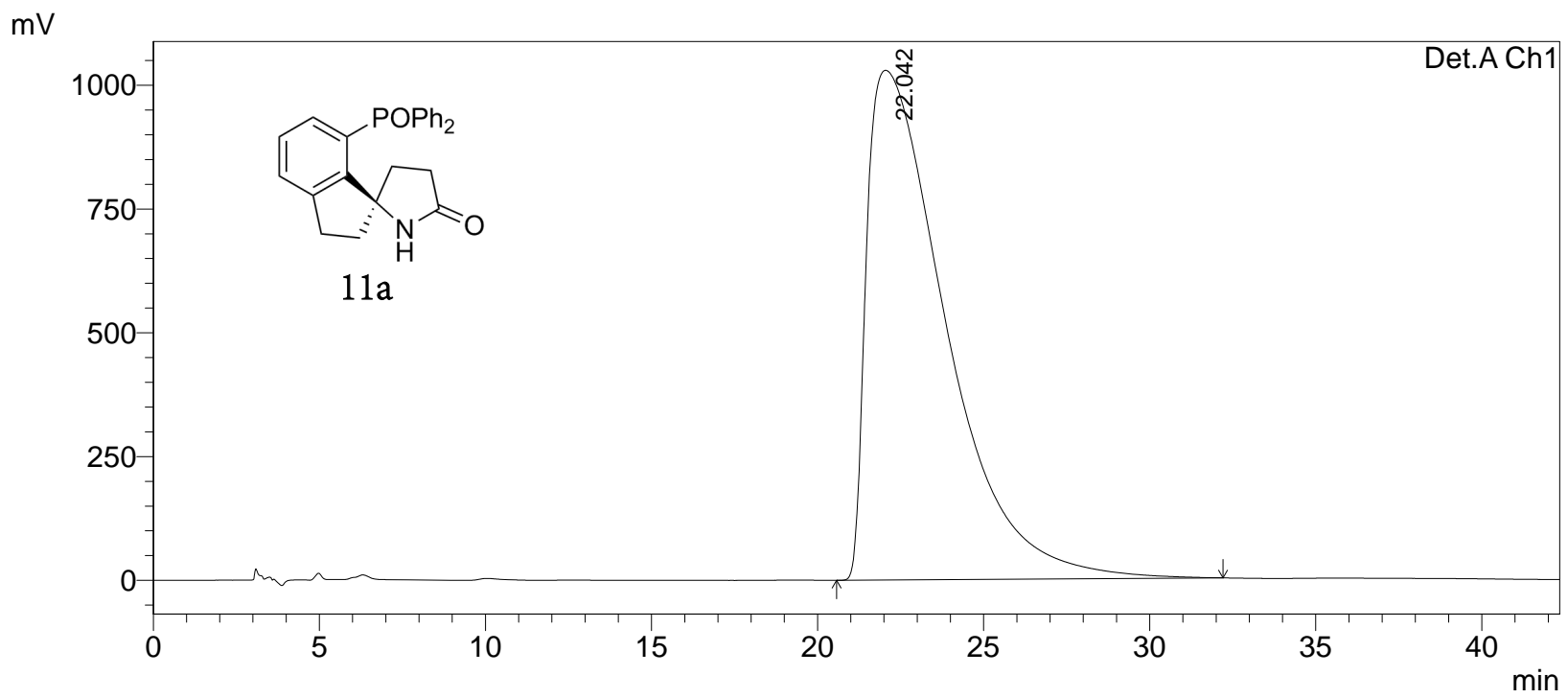

1 Det.A Ch1/210nm

PeakTable

Detector A Ch1 210nm

\begin{tabular}{|r|r|r|r|r|r|}
\hline Peak\# & Ret. Time & \multicolumn{1}{|c|}{ Area } & Height & Area \% & \multicolumn{1}{c|}{ Height \% } \\
\hline 1 & 22.042 & 170406343 & 1029722 & 100.000 & 100.000 \\
\hline Total & & 170406343 & 1029722 & 100.000 & 100.000 \\
\hline
\end{tabular}




\section{$====$ Analysis Report $====$}

Acquired by

E:Idatal901ILSSILSS180331-XX(AD-H 30\% 254 0.3)1.Icd : Admin Sample Name

Sample ID

Vail \# : LSS180331-XX(AD-H 30\% 254 0.3)

Injection Volume $\quad$ : LSS180331-XX(AD-H 30\% 254 0.3)1.Icd

Data File Name

Method File Name

Batch File Name

Report File Name

Data Acquired

: $180327 . \mathrm{lcm}$

: Default.Icr

: 2018/4/19 15:42:17

: 2018/4/19 16:22:23

Data Processed

\section{$<$ Chromatogram $>$}

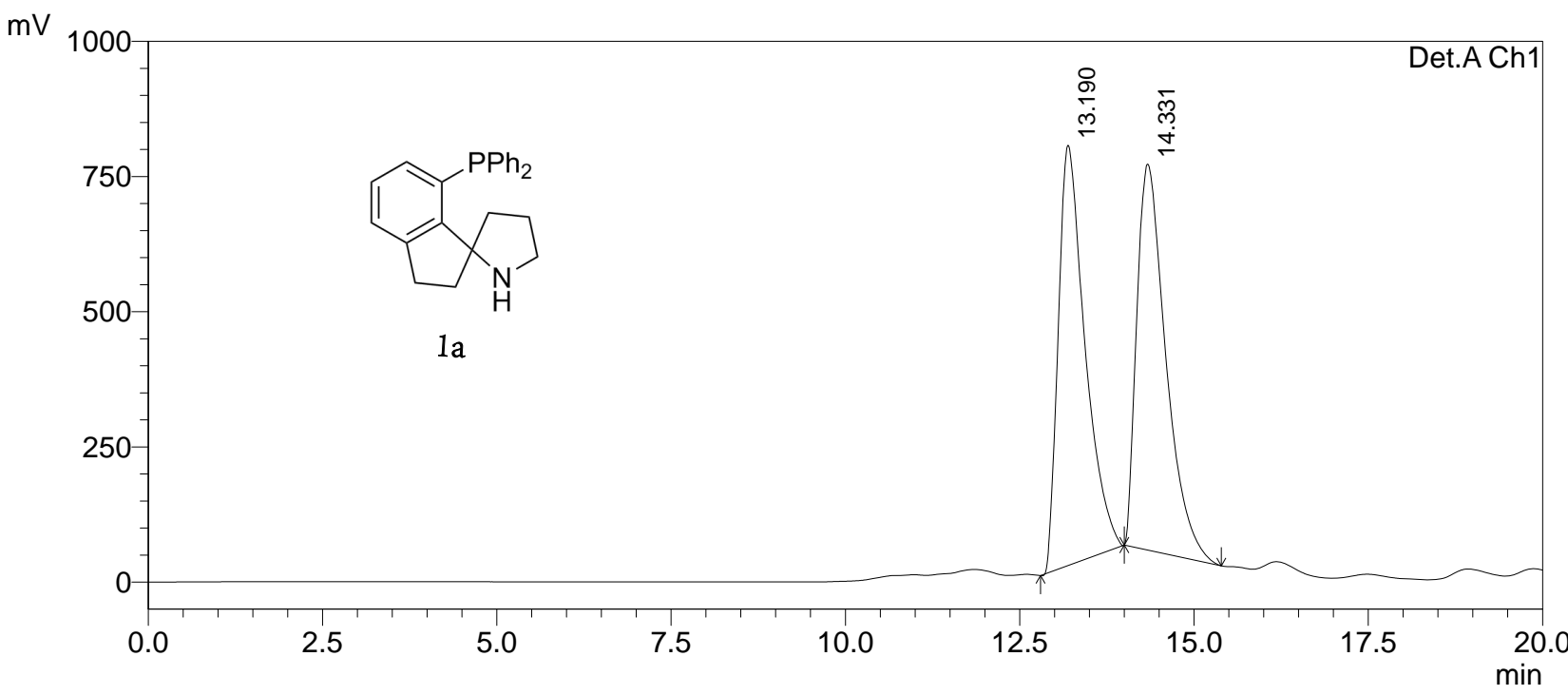

1 Det.A Ch1/254nm

PeakTable

Detector A Ch1 254nm
\begin{tabular}{|r|r|r|r|r|r|}
\hline \multicolumn{1}{|c|}{ Peak\# } & Ret. Time & Area & Height & Area \% & \multicolumn{1}{c|}{ Height \% } \\
\hline 1 & 13.190 & 20774644 & 777411 & 50.055 & 52.134 \\
\hline 2 & 14.331 & 20728981 & 713768 & 49.945 & 47.866 \\
\hline Total & & 41503625 & 1491180 & 100.000 & 100.000 \\
\hline
\end{tabular}




\section{==== Analysis Report ====}

Acquired by

E:Idatal901ILSSILSS180416-3(AD 30\% 254 0.3): Admin

Sample Name

Sample ID

Vail \# : LSS180416-3(AD 30\% 254 0.3)

$: 1 \mathrm{uL}$

Injection Volume $\quad:$ LSS180416-3(AD 30\% 254 0.3)

Data File Name : :180327.Icm

Method File Name

Batch File Name

Report File Name

Data Acquired

:

Default.Icr

: 2018/5/9 15:24:54

: 2018/5/9 15:51:25

Data Processed

\section{$<$ Chromatogram $>$}

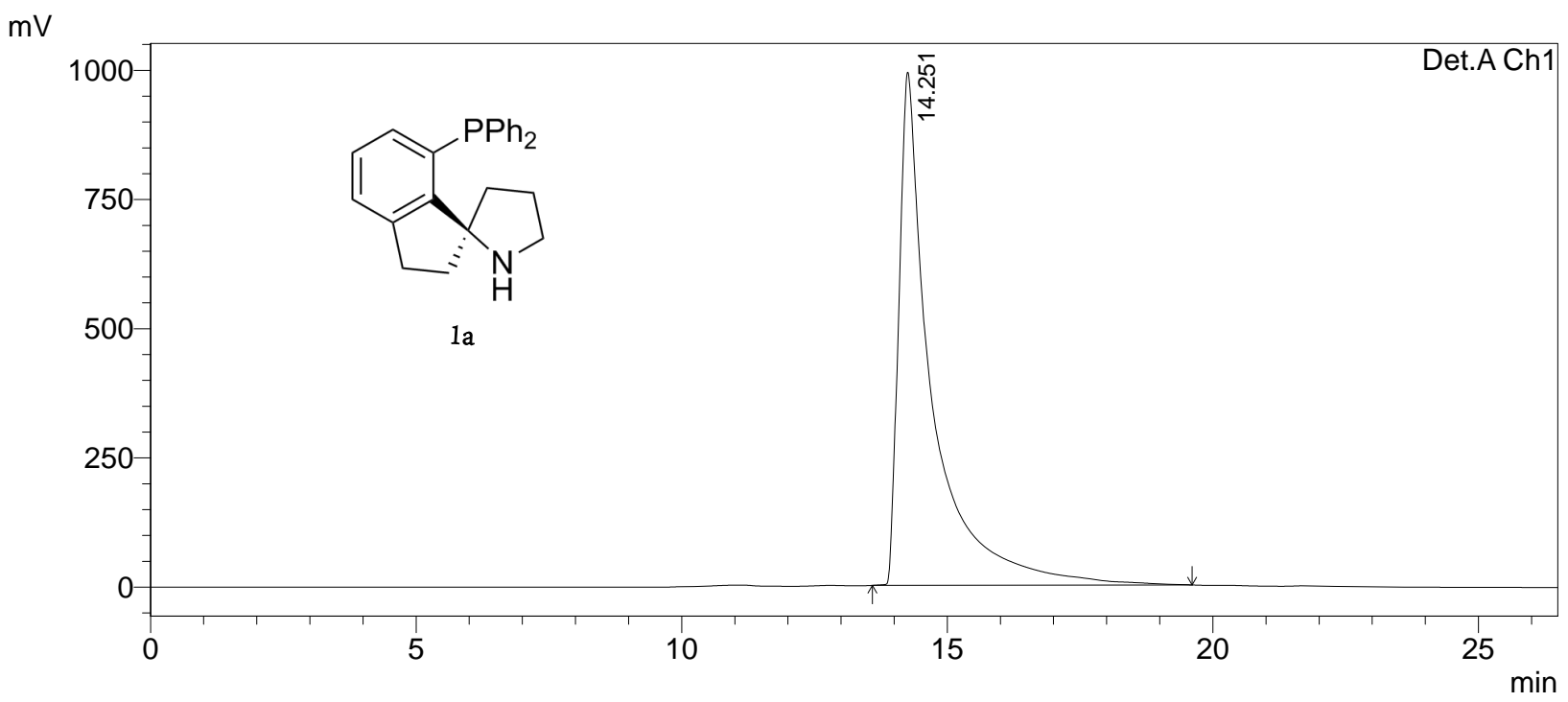

1 Det.A Ch1/254nm

Detector A Ch1 254nm

PeakTable

\begin{tabular}{|r|r|r|r|r|r|}
\hline Peak\# & Ret. Time & Area & \multicolumn{1}{|c|}{ Height } & Area \% & \multicolumn{1}{c|}{ Height \% } \\
\hline 1 & 14.251 & 43140177 & 992998 & 100.000 & 100.000 \\
\hline Total & & 43140177 & 992998 & 100.000 & 100.000 \\
\hline
\end{tabular}




\section{=ニニ= Analysis Report $====$}

Acquired by Sample Name Sample ID

Vail \#

Injection Volume Data File Name Method File Name

Batch File Name

Report File Name

Data Acquired

Data Processed
E:Idatal901ILSSILSS180421-3(AD 5\% 225 1)2.Icd

: Admin

: LSS180421-3(AD 5\% 225 1)

$: 1 \mathrm{uL}$

: LSS180421-3(AD 5\% 225 1)2.Icd

: $180327 . \mathrm{Icm}$

Default.Icr

2018/4/24 17:17:33

$2018 / 4 / 2417: 48: 21$

\section{$<$ Chromatogram >}

$\mathrm{mV}$

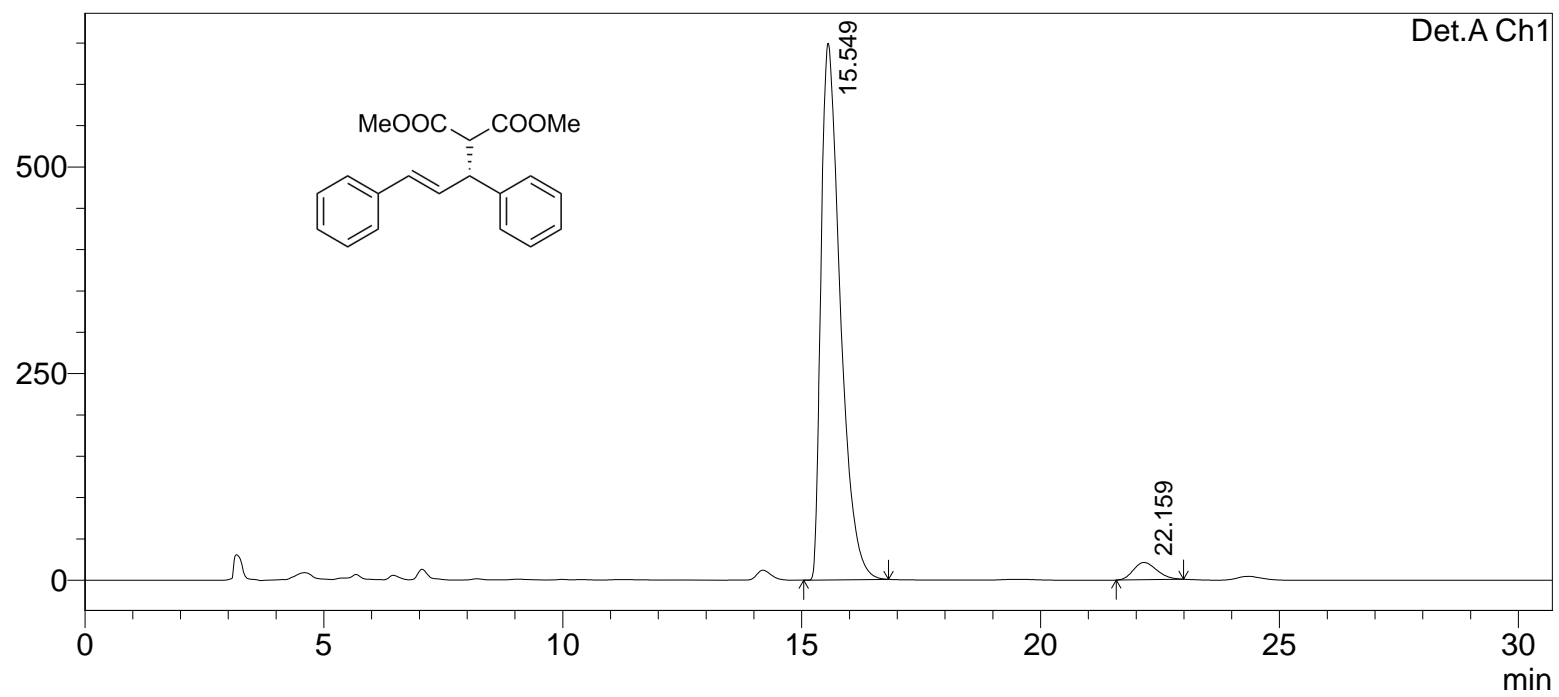

1 Det.A Ch1/225nm

Detector A Ch1 225nm
\begin{tabular}{|r|r|r|r|r|r|}
\hline Peak\# & Ret. Time & Area & Height & Area \% & \multicolumn{1}{c|}{ Height \% } \\
\hline 1 & 15.549 & 18234387 & 649382 & 96.234 & 96.879 \\
\hline 2 & 22.159 & 713643 & 20917 & 3.766 & 3.121 \\
\hline Total & & 18948030 & 670299 & 100.000 & 100.000 \\
\hline
\end{tabular}




\section{$===$ Analysis Report $===$}

Acquired by

Sample Name

Sample ID

Vail \#

Injection Volume

Data File Name

Method File Name

Batch File Name

Report File Name

Data Acquired

Data Processed
E:Idatal901 LSSILSS180419-3(AD-H 10\% 254 1)2.Icd

: Admin

: LSS180419-3(AD-H 10\% 254 1)

: $1 \mathrm{uL}$

: LSS180419-3(AD-H 10\% 254 1)2.Icd

$180327 . \mathrm{lcm}$

: Default.Icr

2018/4/20 16:31:38

: 2018/4/20 16:47:16

\section{$<$ Chromatogram $>$}

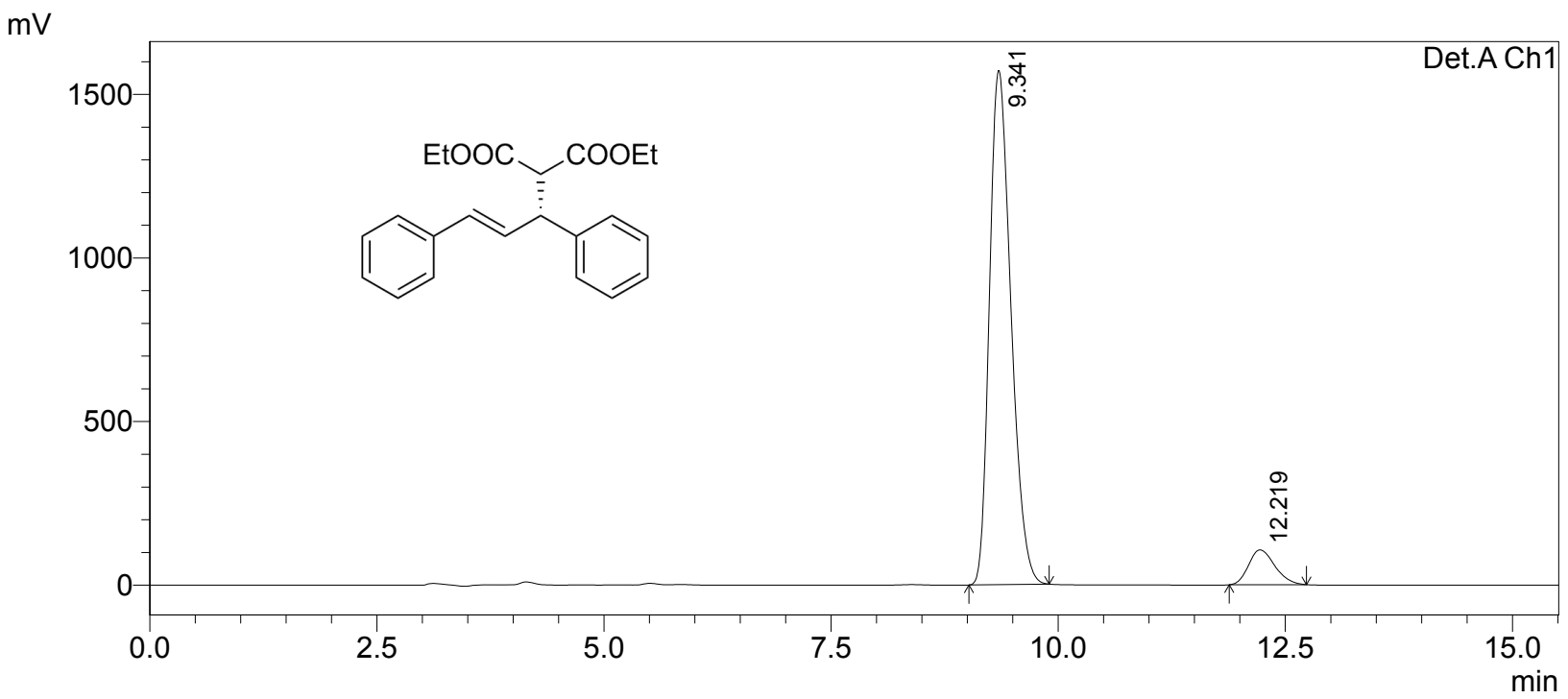

1 Det.A Ch1/254nm

Detector A Ch1 254nm

PeakTable

\begin{tabular}{|r|r|r|r|r|r|}
\hline Peak\# & \multicolumn{1}{|c|}{ Ret. Time } & \multicolumn{1}{|c|}{ Area } & \multicolumn{1}{|c|}{ Height } & \multicolumn{1}{|c|}{ Area \% } & \multicolumn{1}{|c|}{ Height \% } \\
\hline 1 & 9.341 & 25887582 & 1572077 & 92.354 & 93.635 \\
\hline 2 & 12.219 & 2143205 & 106869 & 7.646 & 6.365 \\
\hline Total & & 28030787 & 1678946 & 100.000 & 100.000 \\
\hline
\end{tabular}




\section{==== Analysis Report ====}

Acquired by

E:Idatal901ILSSILSS180719-1-XX(OD 2\% 1)MeOH3.Icd : Admin

Sample Name

Sample ID

Vail \# : LSS180719-1-XX(OD 1.5\% 0.3)MeOH

$: 1 \mathrm{uL}$

Injection Volume $\quad$ : LSS180719-1-XX(OD 2\% 1)MeOH3.Icd

Data File Name

: 180327.lcm

Method File Name

Batch File Name

Report File Name

Data Acquired

: Default.Icr

2018/7/23 15:12:24

: 2018/7/23 17:39:41

Data Processed

\section{$<$ Chromatogram >}

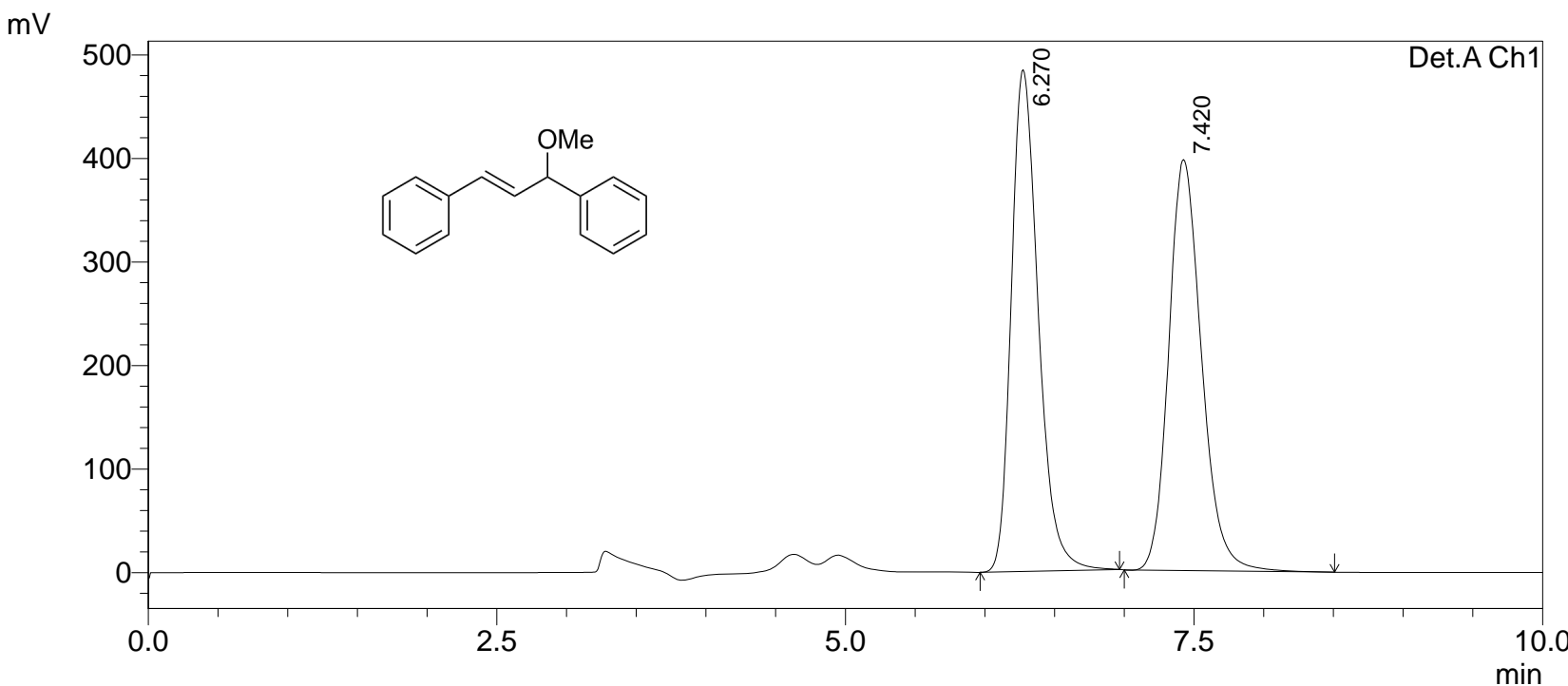

1 Det.A Ch1/260nm

\begin{tabular}{|c|c|c|c|c|c|}
\hline \multicolumn{6}{|c|}{ Detector A Ch } \\
\hline Peak\# & Ret. Time & Area & Height & Area \% & Height \% \\
\hline 1 & 6.270 & 6520172 & 484778 & 50.086 & 54.978 \\
\hline 2 & 7.420 & 6497857 & 396983 & 49.914 & 45.022 \\
\hline Total & & 13018028 & 881761 & 100.000 & 100.000 \\
\hline
\end{tabular}




\section{$== \pm=$ Analysis Report $====$}

Acquired by

E:Idatal901ILSSILSS180603-2(OD 2\% 254 1)2.Icd : Admin

Sample Name

Sample ID

Vail \# : LSS180603-2(OD 2\% 254 1)

$1 \mathrm{uL}$

Injection Volume : : LSS180603-2(OD 2\% 254 1)2.Icd

Data File Name

Method File Name

Batch File Name

Report File Name

Data Acquired

$180327 . \mathrm{Icm}$

Default.Icr

: 2018/6/5 17:00:19

2018/6/5 17:11:50

Data Processed

\section{<Chromatogram>}

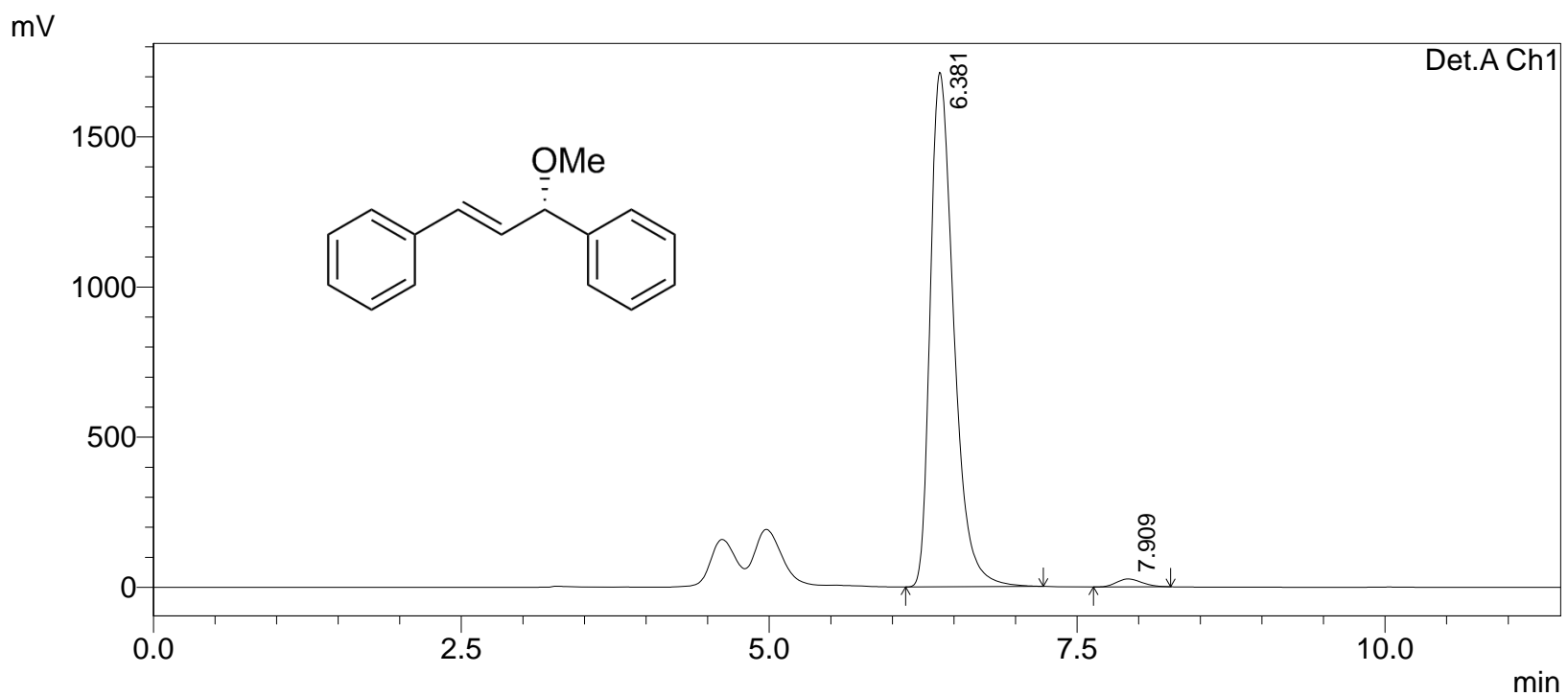

1 Det.A Ch1/254nm

Detector A Ch1 254nm

PeakTable

\begin{tabular}{|r|r|r|r|r|r|}
\hline Peak\# & Ret. Time & \multicolumn{1}{|c|}{ Area } & \multicolumn{1}{|c|}{ Height } & Area \% & \multicolumn{1}{c|}{ Height \% } \\
\hline 1 & 6.381 & 22721695 & 1713903 & 98.347 & 98.474 \\
\hline 2 & 7.909 & 381990 & 26567 & 1.653 & 1.526 \\
\hline Total & & 23103685 & 1740470 & 100.000 & 100.000 \\
\hline
\end{tabular}



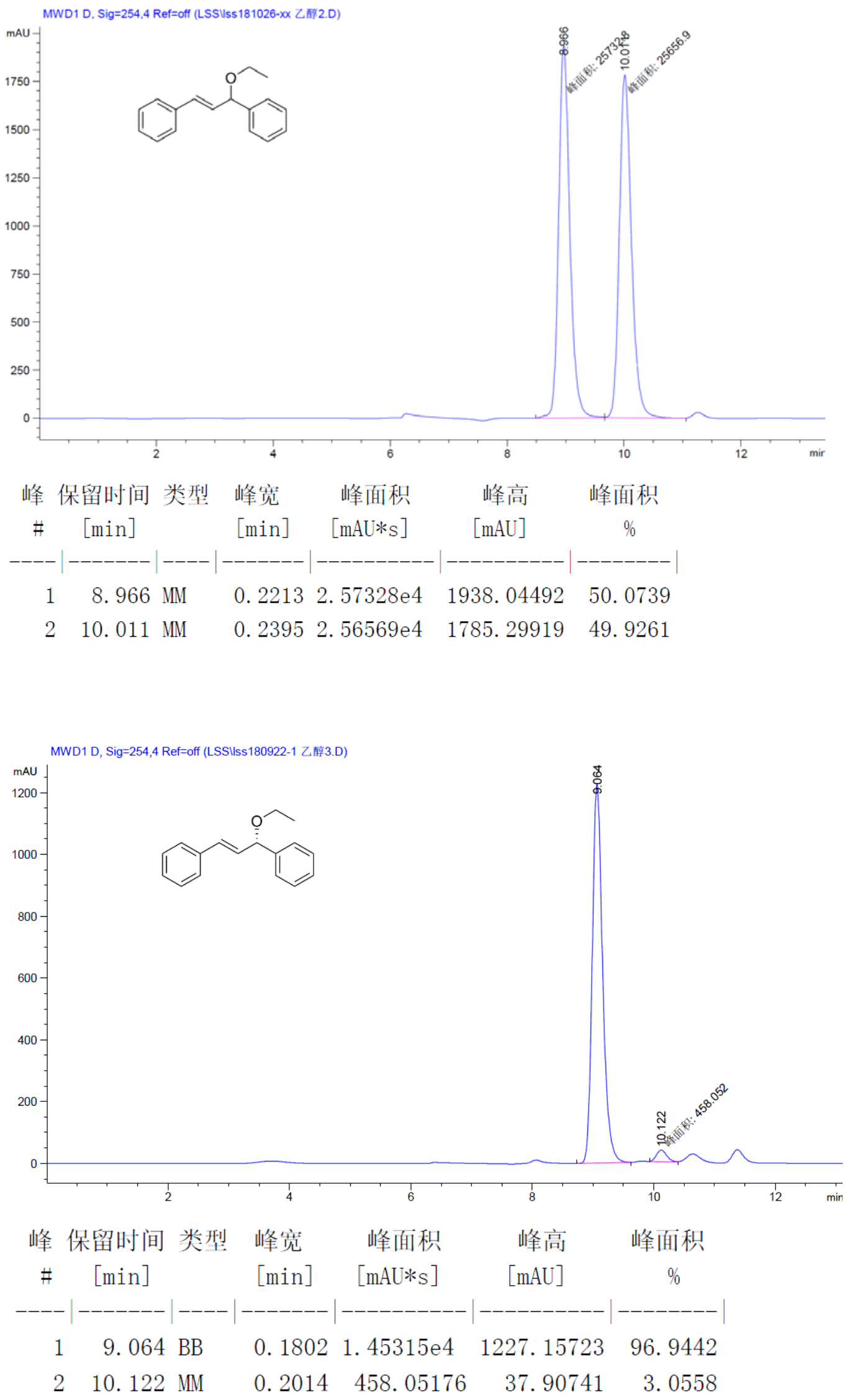

S156 


\section{$====$ Analysis Report $====$}

Acquired by Sample Name Sample ID

Vail \#

E:Idatal901ILSSILSS180912-3-XX(OJ 0.5\% 1 254): Admin : LSS180912-3-XX(OJ 0.5\% 1254$)$

$: 1 \mathrm{uL}$

Injection Volume $\quad$ : LSS180912-3-XX(OJ 0.5\% 1254 )

Data File Name : : 180327.Icm

Method File Name

Batch File Name

Report File Name

Data Acquired :

: Default.lcr

: 2018/9/12 21:01:21

: 2018/9/12 21:24:16

Data Processed

\section{$<$ Chromatogram>}

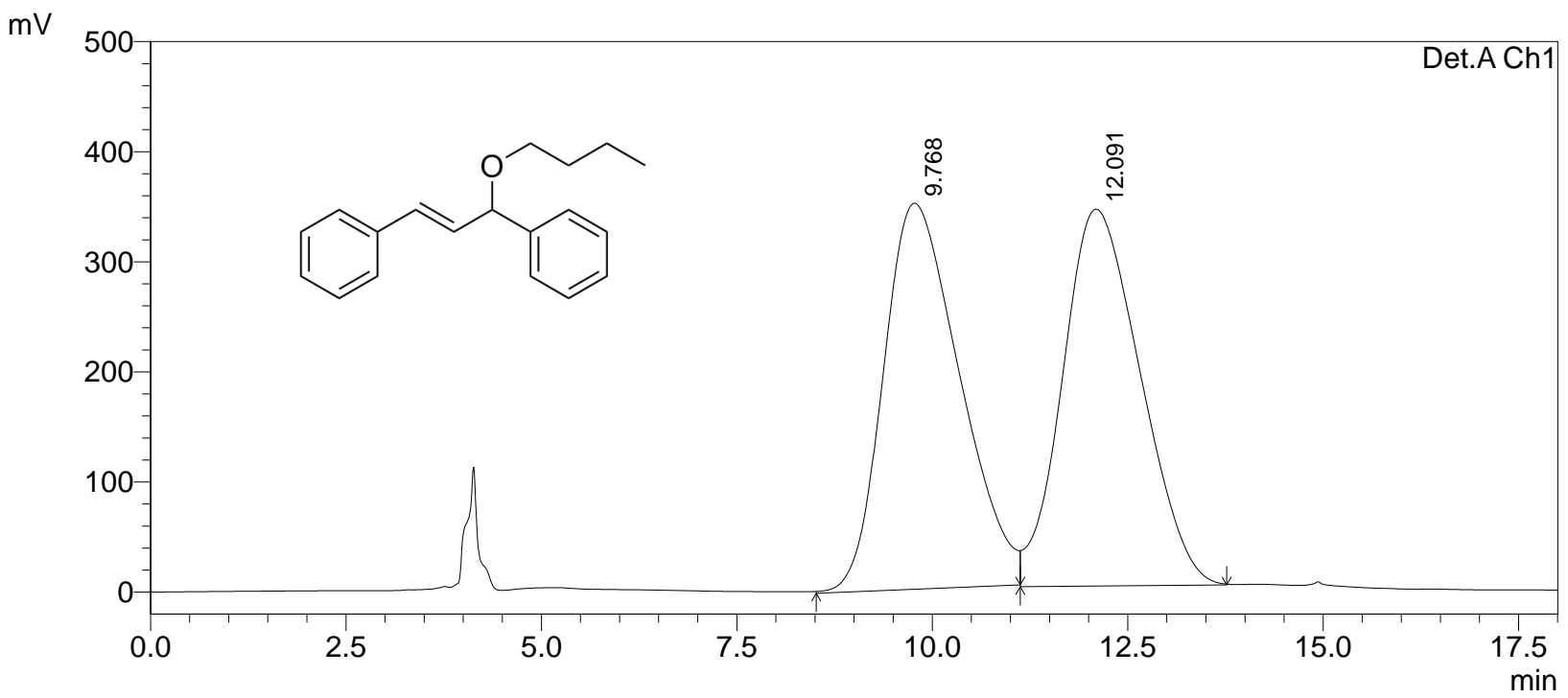

1 Det.A Ch1/254nm

Detector A Ch1 254nm
\begin{tabular}{|r|r|l|r|r|r|}
\hline \multicolumn{1}{|c|}{ Peak\# } & Ret. Time & Area & Height & Area \% & \multicolumn{1}{c|}{ Height $\%$} \\
\hline 1 & 9.768 & 23742464 & 350613 & 50.050 & 50.603 \\
\hline 2 & 12.091 & 23694929 & 342258 & 49.950 & 49.397 \\
\hline Total & & 47437393 & 692871 & 100.000 & 100.000 \\
\hline
\end{tabular}




\section{==== Analysis Report ====}

Acquired by Sample Name Sample ID

Vail \#

E:Idatal901ILSSILSS180912-1(OJ 0.5\% 1 254): Admin : LSS180912-1(OJ 0.5\% 1 254)

$: 1 \mathrm{uL}$

Injection Volume $\quad:$ LSS180912-1(OJ 0.5\% 1254 )

Data File Name : : 180327.Icm

Method File Name

Batch File Name

Report File Name

Data Acquired :

: Default.Icr

: 2018/9/12 21:25:27

: 2018/9/12 21:43:42

Data Processed

\section{$<$ Chromatogram>}

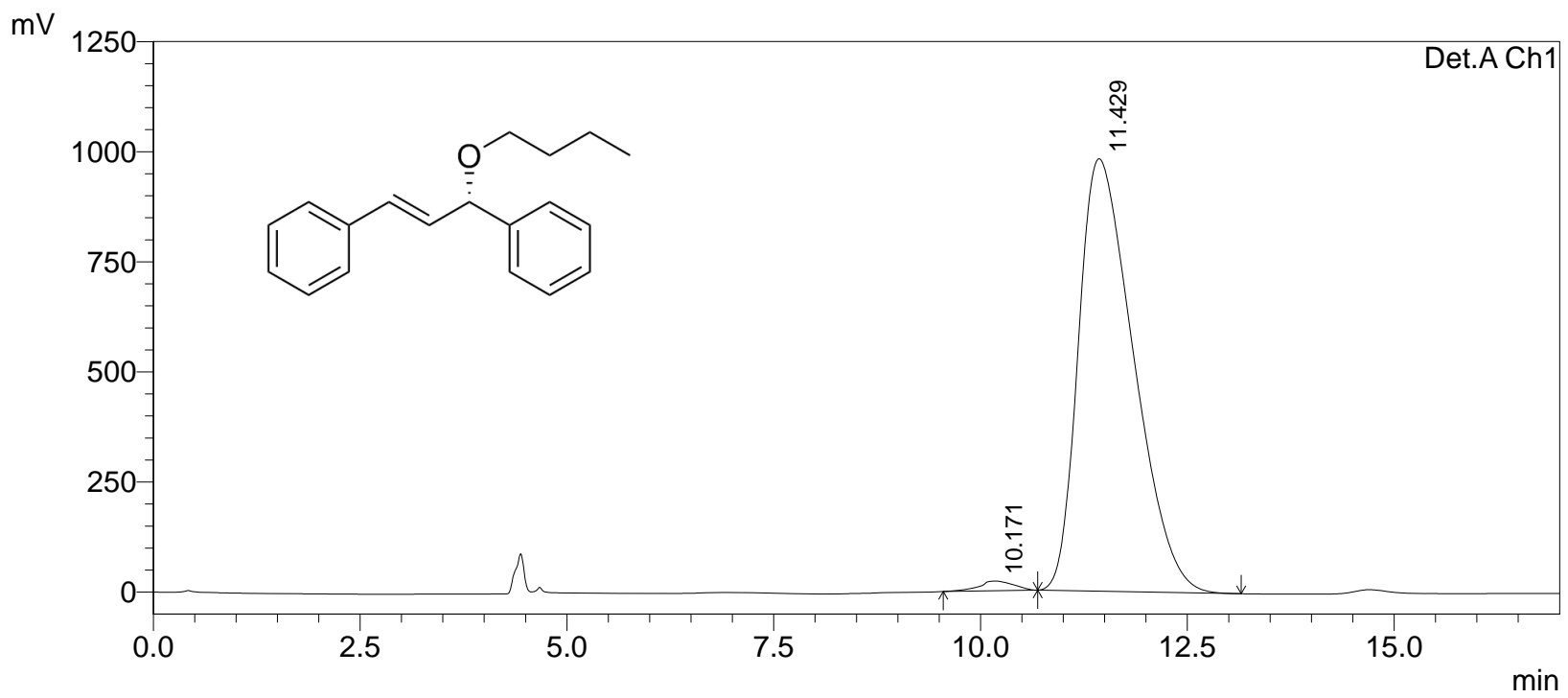

1 Det.A Ch1/254nm

Detector A Ch1 254nm
\begin{tabular}{|r|r|r|r|r|r|}
\hline Peak\# & Ret. Time & Area & Height & Area \% & \multicolumn{1}{c|}{ Height $\%$} \\
\hline 1 & 10.171 & 638177 & 22064 & 1.385 & 2.197 \\
\hline 2 & 11.429 & 45432124 & 982141 & 98.615 & 97.803 \\
\hline Total & & 46070300 & 1004204 & 100.000 & 100.000 \\
\hline
\end{tabular}




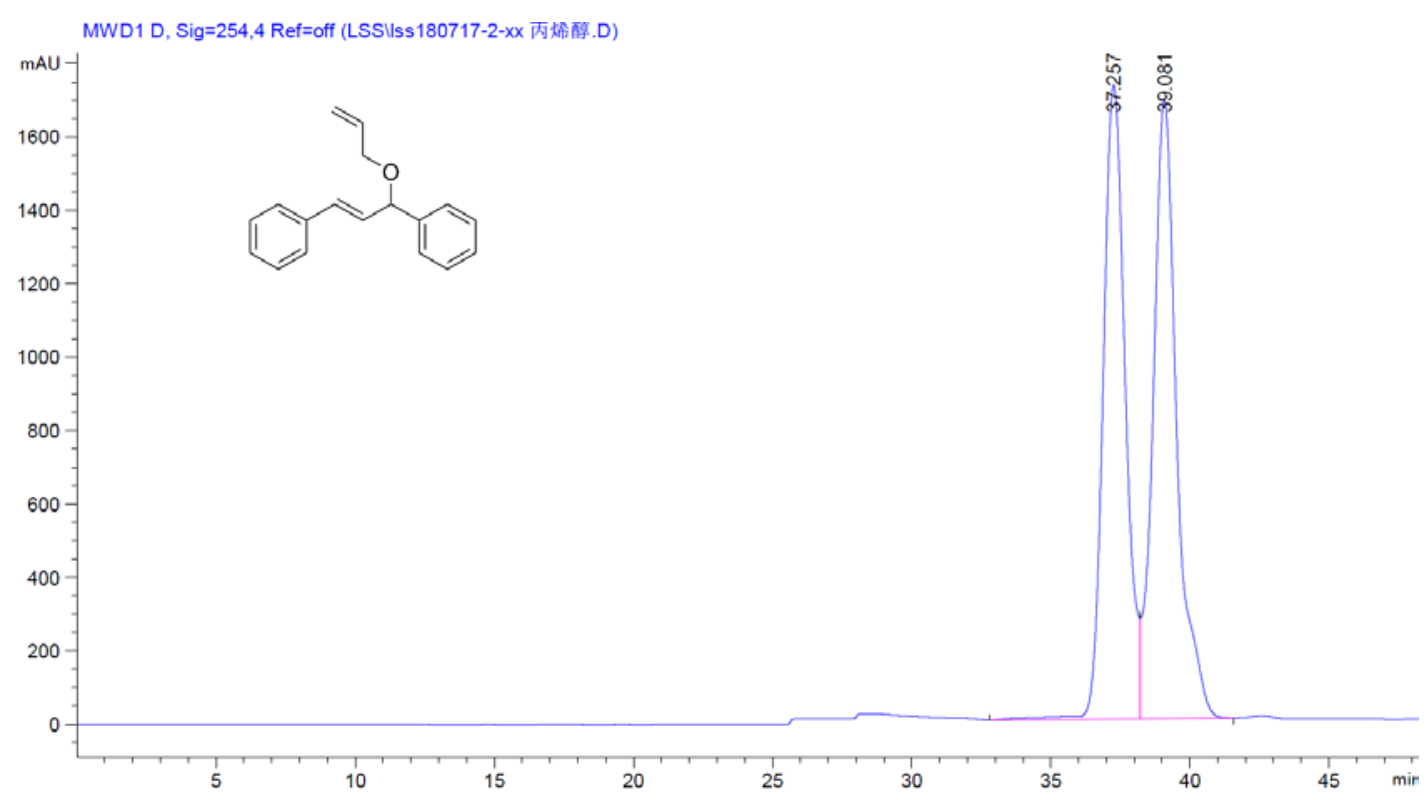

峰 保留时间 类型 峰宽峰面积峰高峰面积

$\begin{array}{ccccccc}\# & {[\mathrm{~min}]} & {[\mathrm{min}]} & {[\mathrm{mAU} * \mathrm{~s}]} & {[\mathrm{mAU}]} & \% \\ -1 & 37.257 & \text { VV R } & 0.8458 & 9.51242 \mathrm{e} 4 & 1728.16785 & 48.9009 \\ 2 & 39.081 & \text { VB } & 0.8805 & 9.94003 \mathrm{e} 4 & 1684.34119 & 51.0991\end{array}$

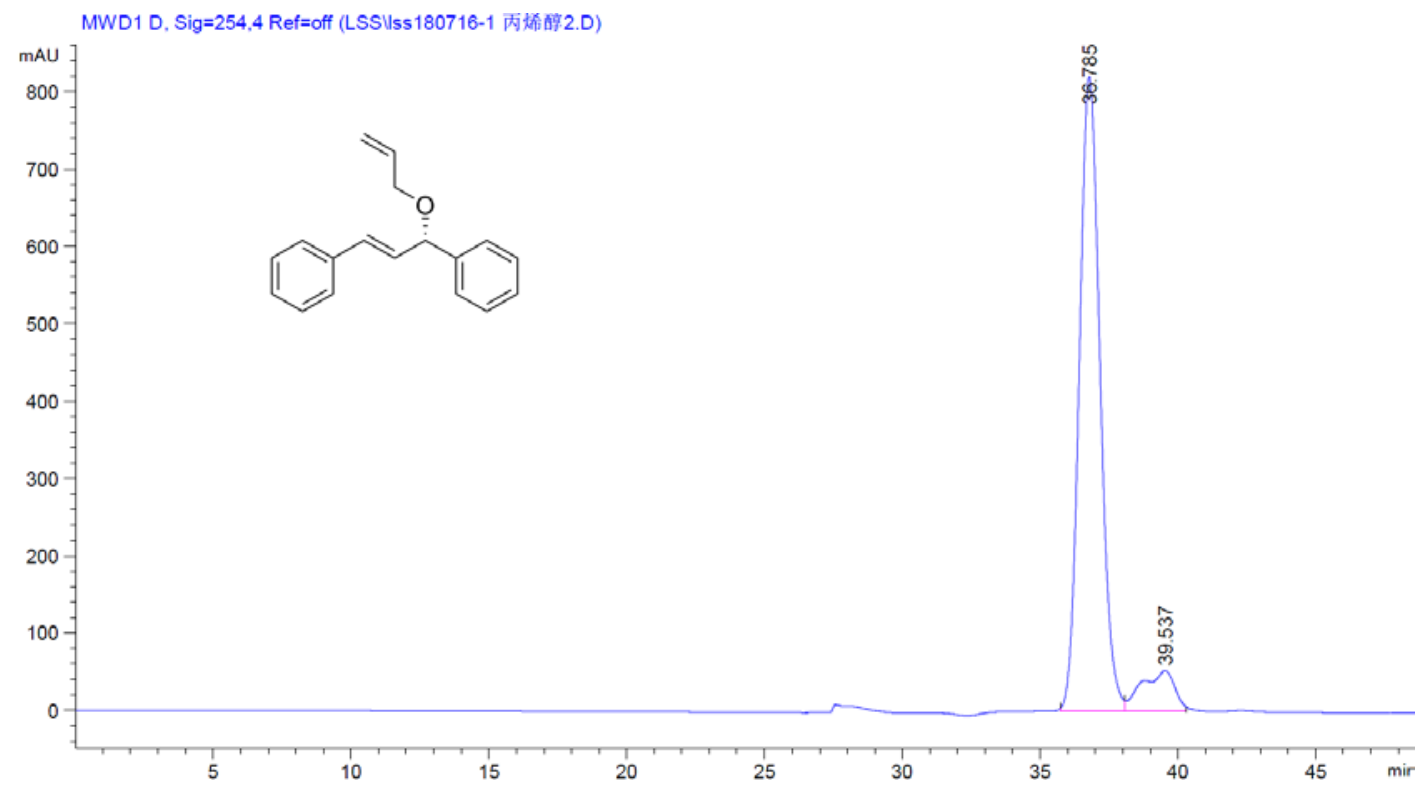

\begin{tabular}{|c|c|c|c|c|c|c|}
\hline $\begin{array}{r}\text { 峰 } \\
\text { \# }\end{array}$ & $\begin{array}{c}\text { 保留时间 } \\
\text { [min] }\end{array}$ & 类型 & $\begin{array}{l}\text { 峰宽 } \\
\text { [min] }\end{array}$ & $\begin{array}{c}\text { 峰面积 } \\
\text { [mAU*s] }\end{array}$ & $\begin{array}{l}\text { 峰高 } \\
\text { [mAU] }\end{array}$ & $\begin{array}{c}\text { 峰面积 } \\
\%\end{array}$ \\
\hline 1 & 36. 785 & MF & 0.8704 & 4. $28488 \mathrm{e} 4$ & 820.51752 & 90.8183 \\
\hline 2 & 39.537 & $\mathrm{FY}$ & 1. 3877 & 4331.97852 & 52.02971 & 9. 1817 \\
\hline
\end{tabular}




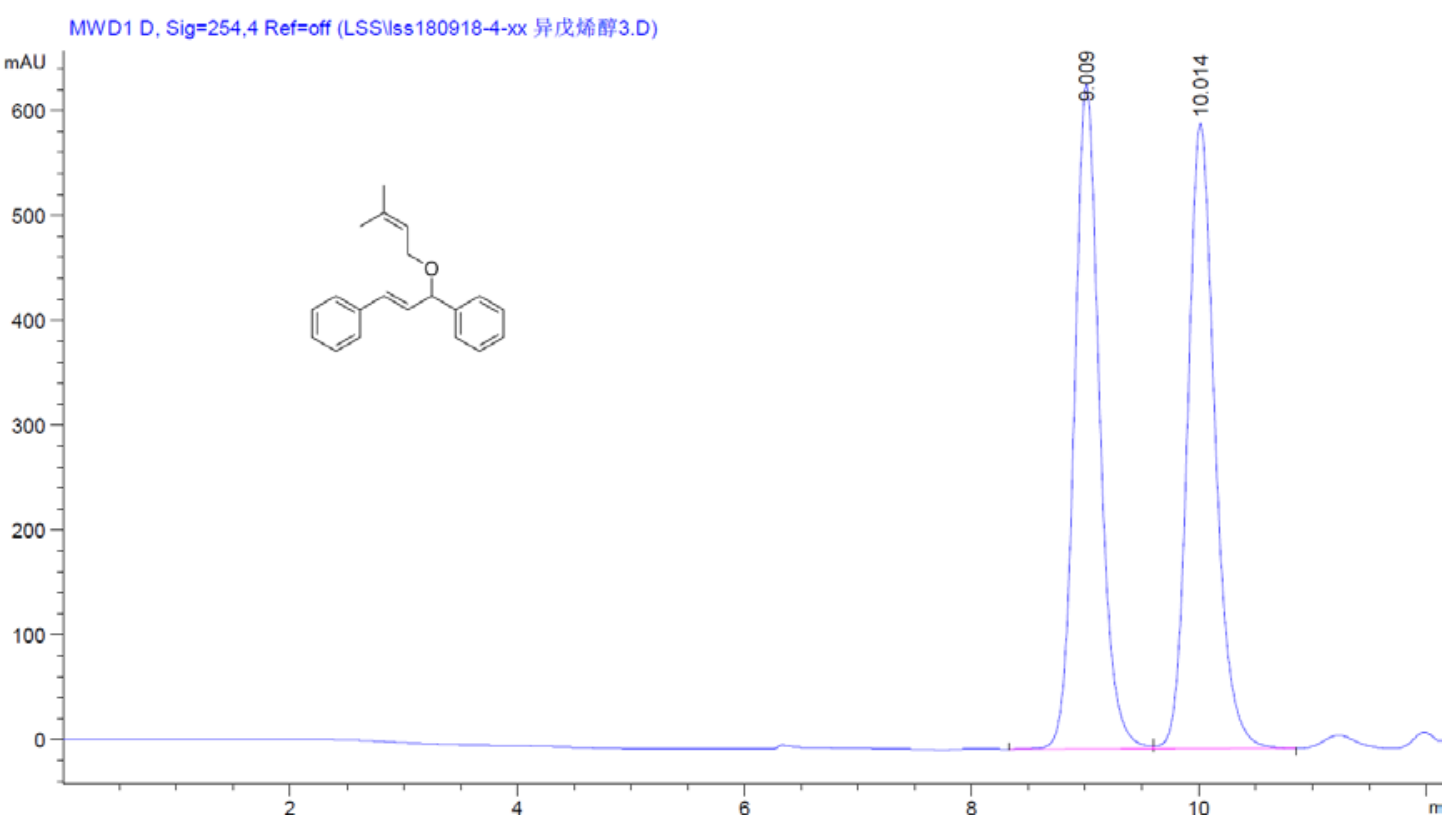

峰保留时间 类型峰宽峰面积峰高峰面积

$\begin{array}{rrcccc}\# & \text { [min }] & {[\mathrm{min}]} & {[\mathrm{mAU} * \mathrm{~s}]} & {[\mathrm{mAU}]} & \% \\ 1 & 9.009 \mathrm{BV} & 0.2331 & 9732.02734 & 633.94232 & 49.4456 \\ 2 & 10.014 \mathrm{VB} & 0.2547 & 9950.27734 & 596.14136 & 50.5544\end{array}$

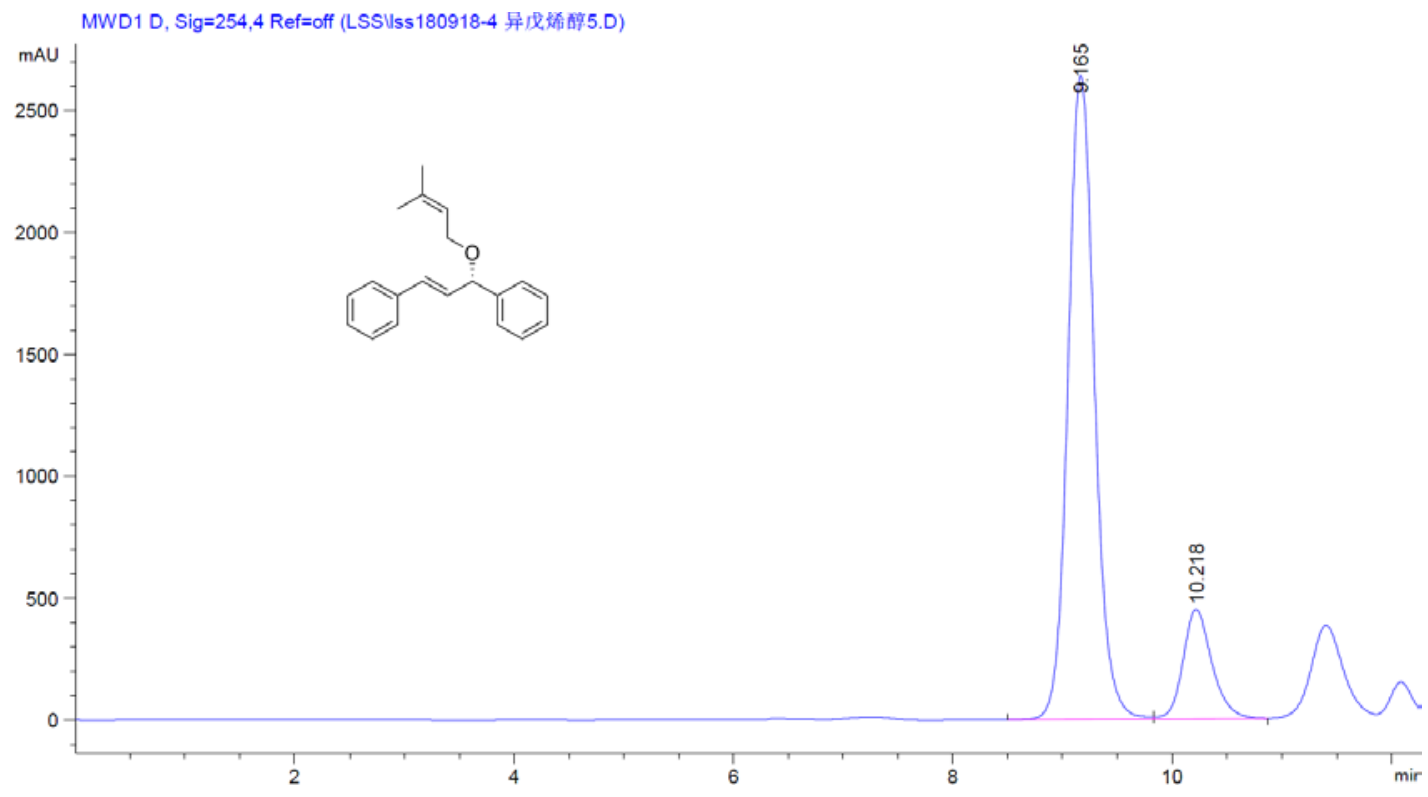

峰 保留时间 类型 峰宽峰面积峰高峰面积

$\begin{array}{cccccc}\# & {[\mathrm{~min}]} & {[\mathrm{min}]} & {[\mathrm{mAU} * \mathrm{~s}]} & {[\mathrm{mAU}]} & \% \\ -1 & 9.165 \mathrm{BV} & 0.2603 & 4.44138 \mathrm{e} 4 & 2639.77759 & 84.4057 \\ 2 & 10.218 \text { VB } & 0.2766 & 8205.63086 & 450.20187 & 15.5943\end{array}$




\section{$== \pm=$ Analysis Report $====$}

Acquired by

E:Idatal901ILSSILSS180719-3-XX(OD 1.5\% 254 0.3)1.Icd : Admin

Sample Name

Sample ID

Vail \# : LSS180719-3-XX(OD 1.5\% 254 0.3)

Injection Volume $\quad$ : LSS180719-3-XX(OD 1.5\% 254 0.3)1.Icd

Data File Name

Method File Name

Batch File Name

Report File Name

Data Acquired

$180327 . \mathrm{Icm}$

Default.lcr

: 2018/8/4 9:36:52

: 2018/8/4 10:20:22

Data Processed

\section{<Chromatogram>}

$\mathrm{mV}$

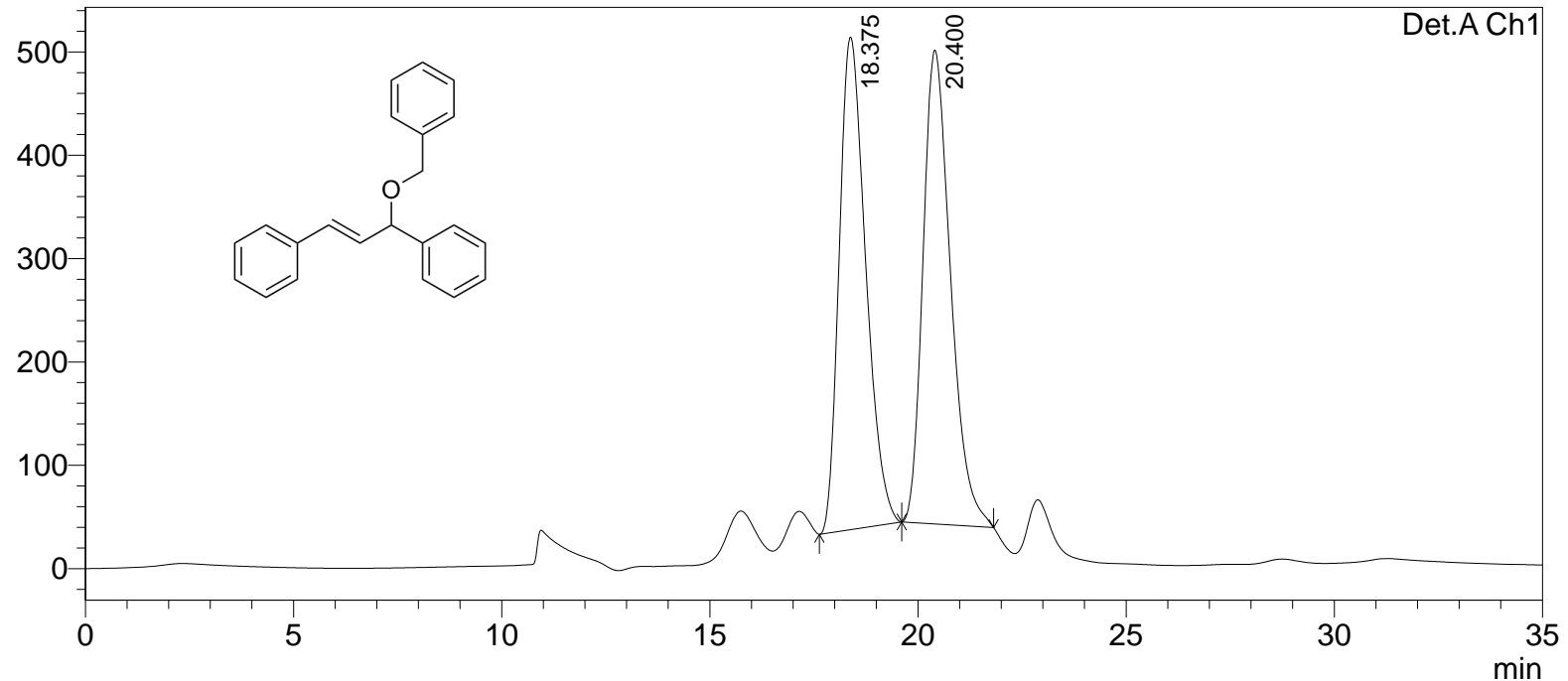

1 Det.A Ch1/254nm

\begin{tabular}{|c|c|c|c|c|c|}
\hline \multirow{2}{*}{\multicolumn{6}{|c|}{ Detector A Ch1 254nm }} \\
\hline & & & & & \\
\hline Peak\# & Ret. Time & Area & Height & Area \% & Height \% \\
\hline 1 & 18.375 & 21048403 & 476730 & 49.930 & 50.971 \\
\hline 2 & 20.400 & 21107154 & 458571 & 50.070 & 49.029 \\
\hline Total & & 42155558 & 935300 & 100.000 & 100.000 \\
\hline
\end{tabular}




\section{==== Analysis Report ====}

Acquired by Sample Name Sample ID

Vail \#

E:Idatal901ILSSILSS180511-4-DCM(OD 1.5\% 254 0.3)6.Icd : Admin : LSS180511-4-DCM(OD 1.5\% 254 0.3)

$: 1 \mathrm{uL}$

Injection Volume $\quad$ : LSS180511-4-DCM(OD 1.5\% 254 0.3)6.Icd

Data File Name

Method File Name

Batch File Name

Report File Name

Data Acquired

$180327 . \mathrm{Icm}$

Default.Icr

: 2018/5/14 15:38:56

: 2018/5/14 16:10:43

Data Processed

\section{$<$ Chromatogram $>$}

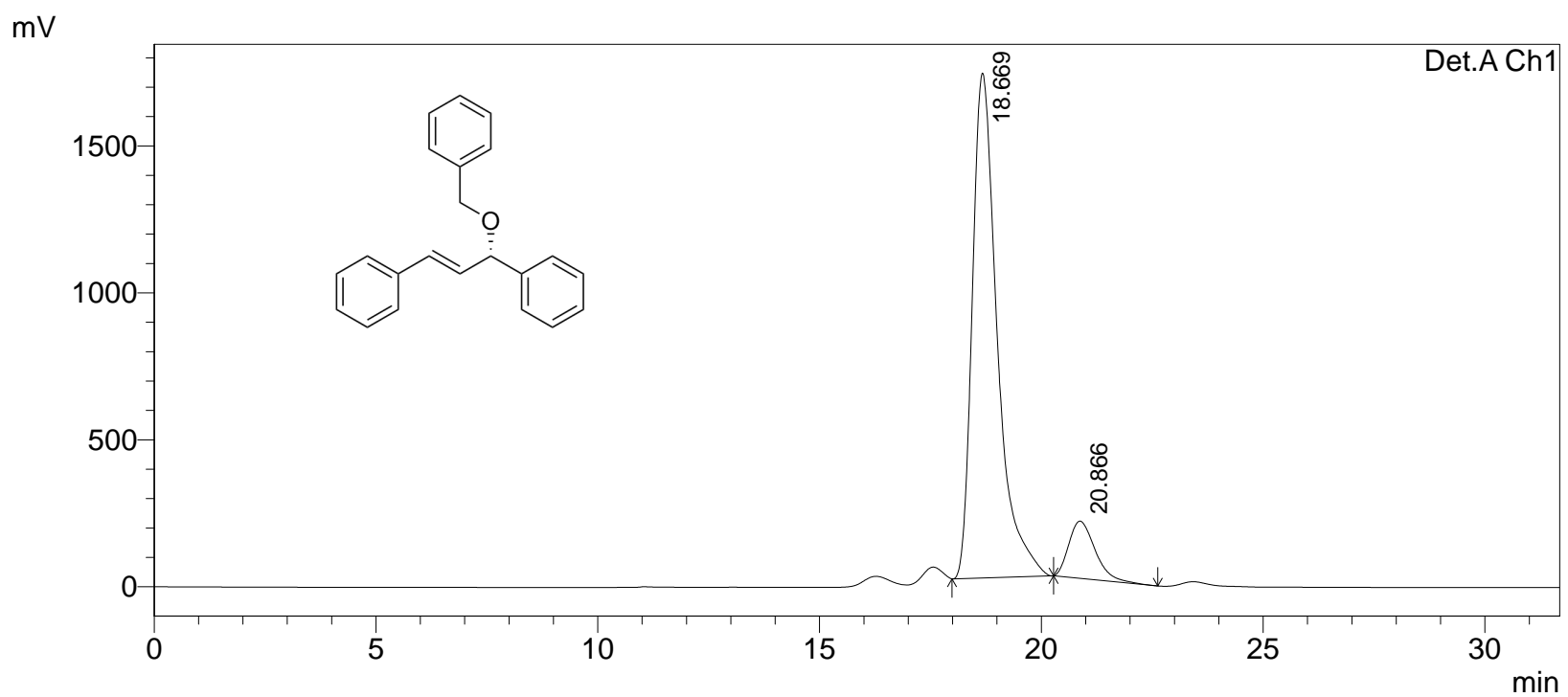

1 Det.A Ch1/254nm

PeakTable

Detector A Ch1 254nm

\begin{tabular}{|r|r|r|r|r|r|}
\hline Peak\# & \multicolumn{1}{|c|}{ Ret. Time } & \multicolumn{1}{c|}{ Area } & \multicolumn{1}{c|}{ Height } & Area \% & \multicolumn{1}{c|}{ Height \% } \\
\hline 1 & 18.669 & 66860243 & 1718212 & 89.234 & 89.815 \\
\hline 2 & 20.866 & 8067033 & 194838 & 10.766 & 10.185 \\
\hline Total & & 74927276 & 1913050 & 100.000 & 100.000 \\
\hline
\end{tabular}




\section{===+ Analysis Report ====}

Acquired by Sample Name Sample ID

Vail \#

E:Idatal901LSSILSS180918-5-XX(OJ 1\% 1254 ) : Admin : LSS180918-5-XX(OJ 1\% 1254 )

: $1 \mathrm{uL}$

Injection Volume $\quad$ : LSS180918-5-XX(OJ 1\% 1254 )

Data File Name : $180327 . I \mathrm{~cm}$

Method File Name :

Batch File Name : Default.lcr

Report File Name $\quad:$ :2018/9/20 13:48:04

Data Acquired : : 2018/9/20 14:38:23

Data Processed

\section{$<$ Chromatogram $>$}

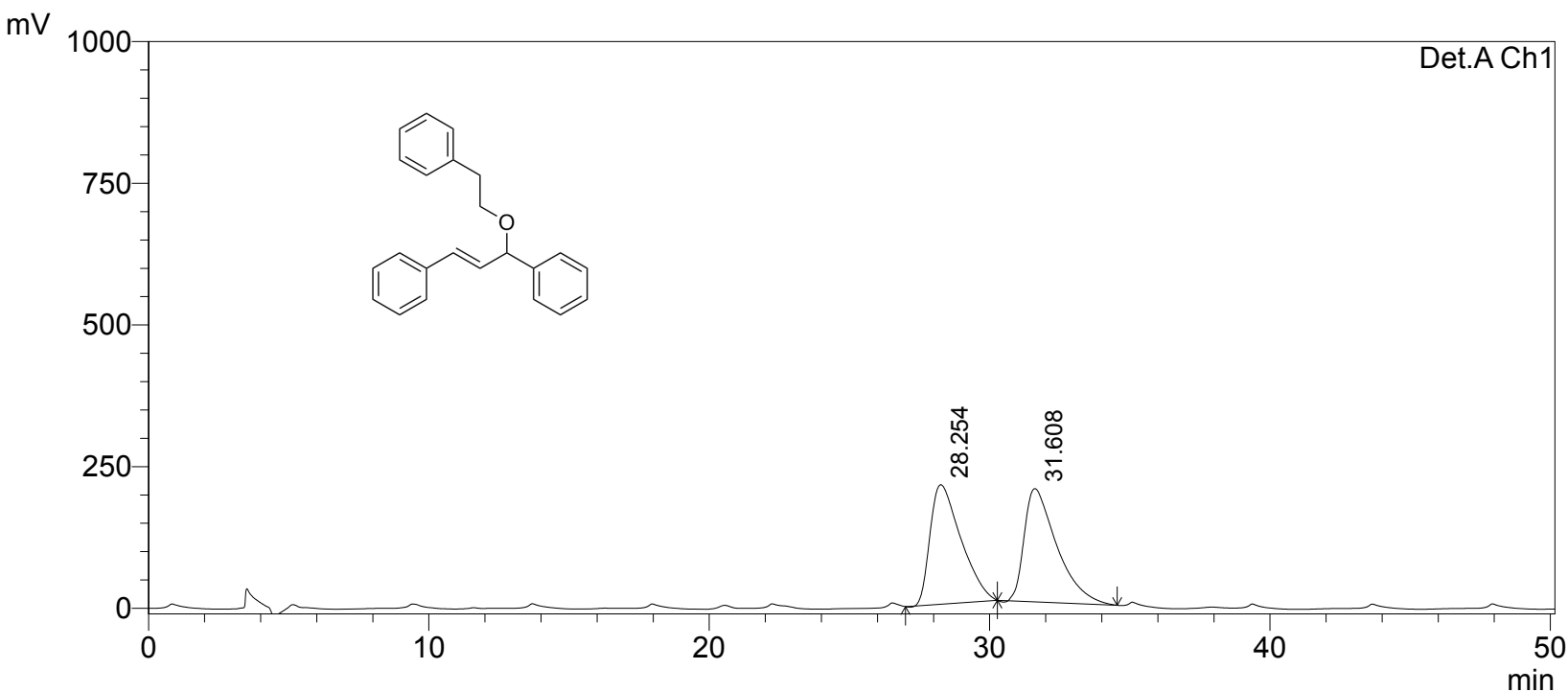

1 Det.A Ch1/254nm

Detector A Ch1 254nm

PeakTable

\begin{tabular}{|r|r|r|r|r|r|}
\hline \multicolumn{1}{|c|}{ Peak\# } & Ret. Time & Area & Height & Area \% & \multicolumn{1}{|c|}{ Height \% } \\
\hline 1 & 28.254 & 16149547 & 210904 & 49.981 & 51.337 \\
\hline 2 & 31.608 & 16161818 & 199918 & 50.019 & 48.663 \\
\hline Total & & 32311364 & 410822 & 100.000 & 100.000 \\
\hline
\end{tabular}




\section{$====$ Analysis Report $====$}

Acquired by Sample Name Sample ID

Vail \#

E:Idatal901ILSSILSS180918-2(OJ 1\% 1 254) : Admin : LSS180918-2(OJ 1\% 1 254)

$: 1 \mathrm{uL}$

Injection Volume $\quad$ : LSS180918-2(OJ 1\% 1254$)$

Data File Name : : 180327.Icm

Method File Name

Batch File Name

Report File Name

Data Acquired $:$

: Default.Icr

: 2018/9/20 15:44:25

: 2018/9/20 16:50:03

Data Processed

\section{$<$ Chromatogram >}

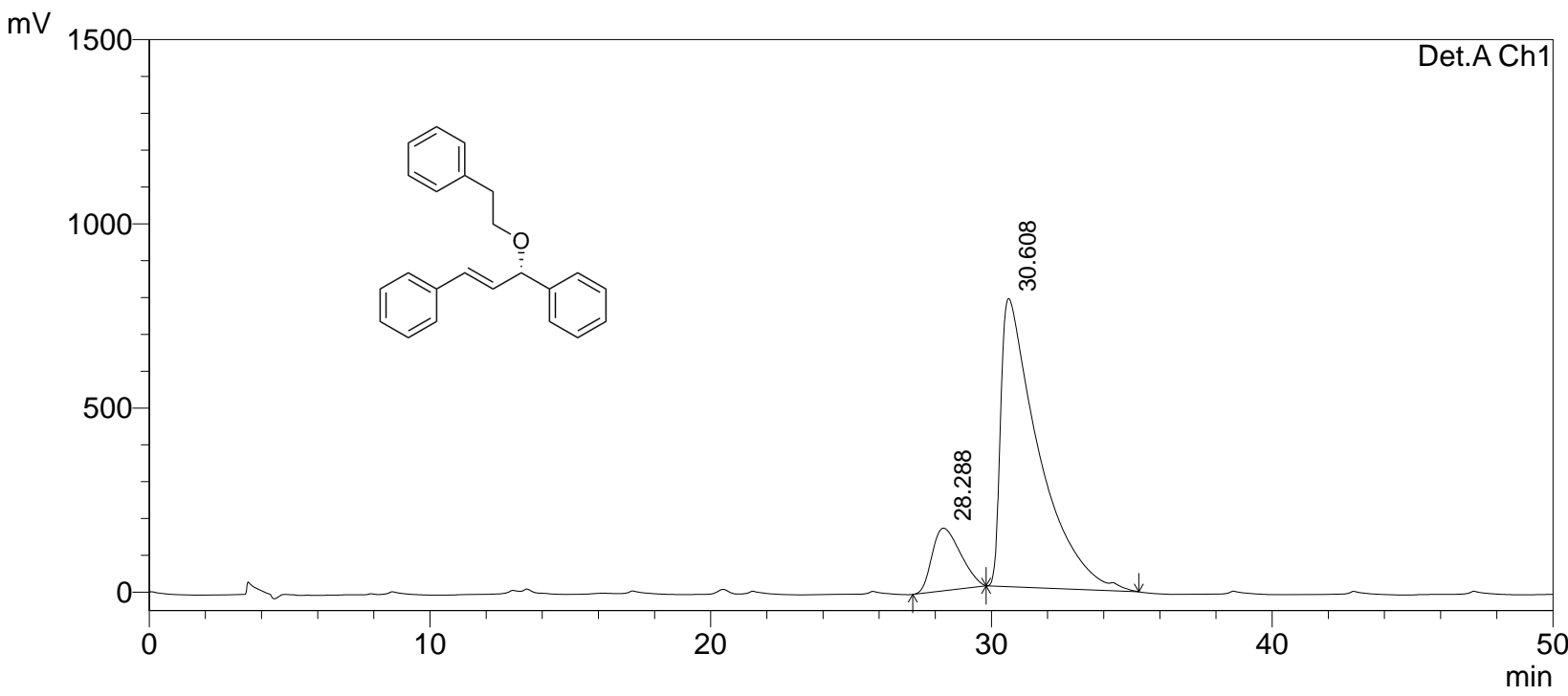

1 Det.A Ch1/254nm

PeakTable

Detector A Ch1 254nm

\begin{tabular}{|r|r|r|r|r|r|}
\hline \multicolumn{1}{|c|}{ Peak\# } & Ret. Time & Area & \multicolumn{1}{|c|}{ Height } & \multicolumn{1}{c|}{ Area \% } & \multicolumn{1}{c|}{ Height \% } \\
\hline 1 & 28.288 & 11953037 & 170148 & 14.024 & 17.864 \\
\hline 2 & 30.608 & 73282588 & 782341 & 85.976 & 82.136 \\
\hline Total & & 85235626 & 952489 & 100.000 & 100.000 \\
\hline
\end{tabular}




\section{=ニニ= Analysis Report $====$}

Acquired by Sample Name Sample ID

Vail \#

Injection Volume

Data File Name

Method File Name

E:Idatal901ILSSILSS180912-4-XX(OJ 30\% 1 254)

: Admin

: LSS180912-4-XX(OJ 30\% 1 254)

:

$1 \mathrm{uL}$

: LSS180912-4-XX(OJ 30\% 1 254) :

Batch File Name

Report File Name

Data Acquired $180327 . \mathrm{Icm}$

: Default.lcr

:2018/9/13 15:24:00

: 2018/9/13 16:06:16

\section{$<$ Chromatogram >}

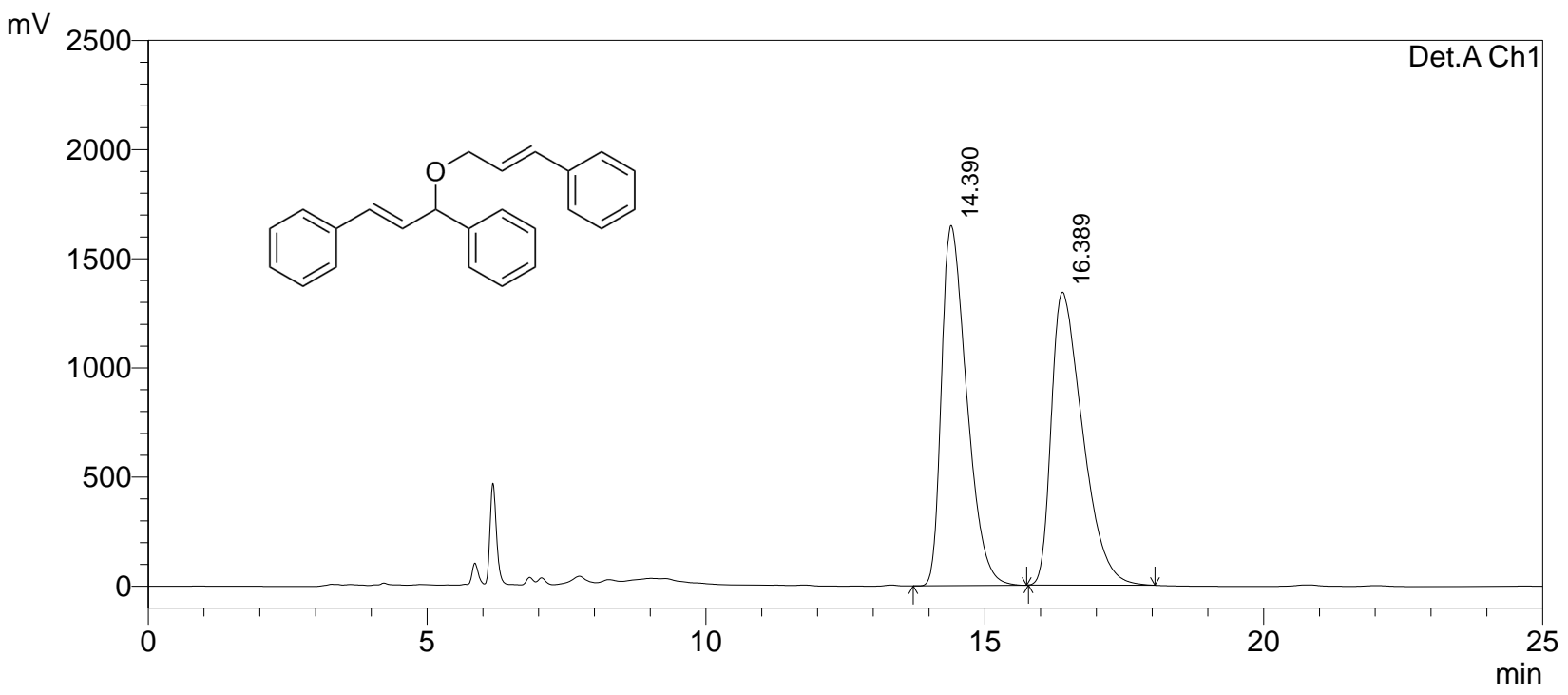

1 Det.A Ch1/254nm

PeakTable

Detector A Ch1 254nm
\begin{tabular}{|r|r|r|r|r|r|}
\hline Peak\# & Ret. Time & \multicolumn{1}{|c|}{ Area } & Height & Area \% & \multicolumn{1}{c|}{ Height \% } \\
\hline 1 & 14.390 & 51841718 & 1649289 & 49.767 & 55.145 \\
\hline 2 & 16.389 & 52327565 & 1341553 & 50.233 & 44.855 \\
\hline Total & & 104169283 & 2990842 & 100.000 & 100.000 \\
\hline
\end{tabular}




\section{$====$ Analysis Report $====$}

Acquired by Sample Name Sample ID

Vail \#

\section{E:Idatal901LSSILSS180912-2(OJ 30\% 1 254) : Admin} : LSS180912-2(OJ 30\% 1 254)

$: 1 \mathrm{uL}$

Injection Volume $\quad$ : LSS180912-2(OJ 30\% 1254$)$

Data File Name

Method File Name

Batch File Name

Report File Name

Data Acquired

: $180327 . \mathrm{lcm}$

: Default.Icr

: 2018/9/13 16:28:02

: 2018/9/13 16:51:05

Data Processed

\section{$<$ Chromatogram $>$}

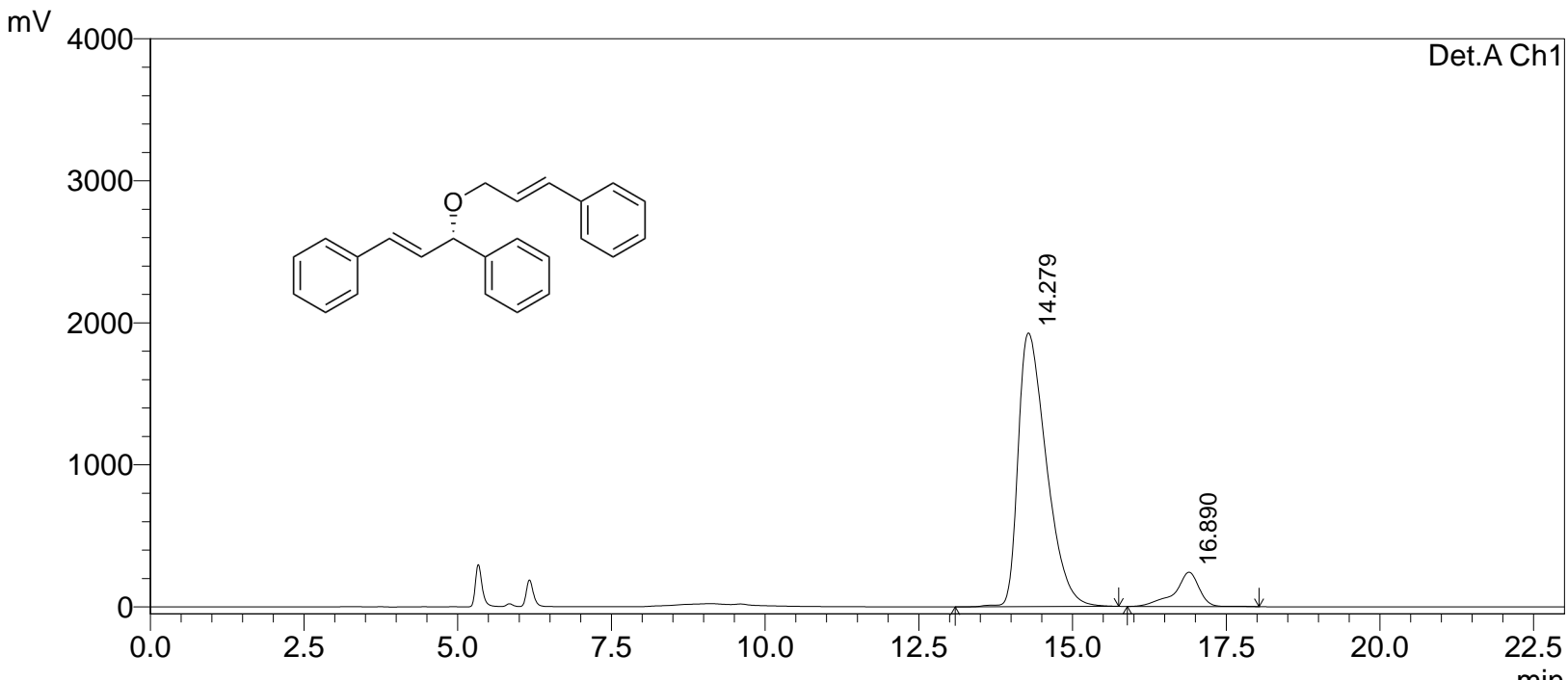

1 Det.A Ch1/254nm

Detector A Ch1 254nm
\begin{tabular}{|r|r|r|r|r|r|}
\hline Peak\# & Ret. Time & \multicolumn{1}{|c|}{ Area } & Height & Area $\%$ & \multicolumn{1}{|c|}{ Height $\%$} \\
\hline 1 & 14.279 & 61682873 & 1927062 & 90.133 & 88.832 \\
\hline 2 & 16.890 & 6752633 & 242269 & 9.867 & 11.168 \\
\hline Total & & 68435506 & 2169331 & 100.000 & 100.000 \\
\hline
\end{tabular}




\section{$====$ Analysis Report $====$}

Acquired by Sample Name Sample ID

Vail \#

E:Idatal901ILSSILSS180717-3-XX(OD 10\% 1): Admin : LSS180716-2-XX(OD 10\% 1)

$: 1 \mathrm{uL}$

Injection Volume $\quad:$ LSS180717-3-XX(OD 10\% 1)

Data File Name

Method File Name

Batch File Name

Report File Name

Data Acquired

180327.Icm

: Default.Icr

: 2018/7/19 21:32:15

: 2018/7/19 22:42:50

Data Processed

\section{$<$ Chromatogram $>$}

$\mathrm{mV}$

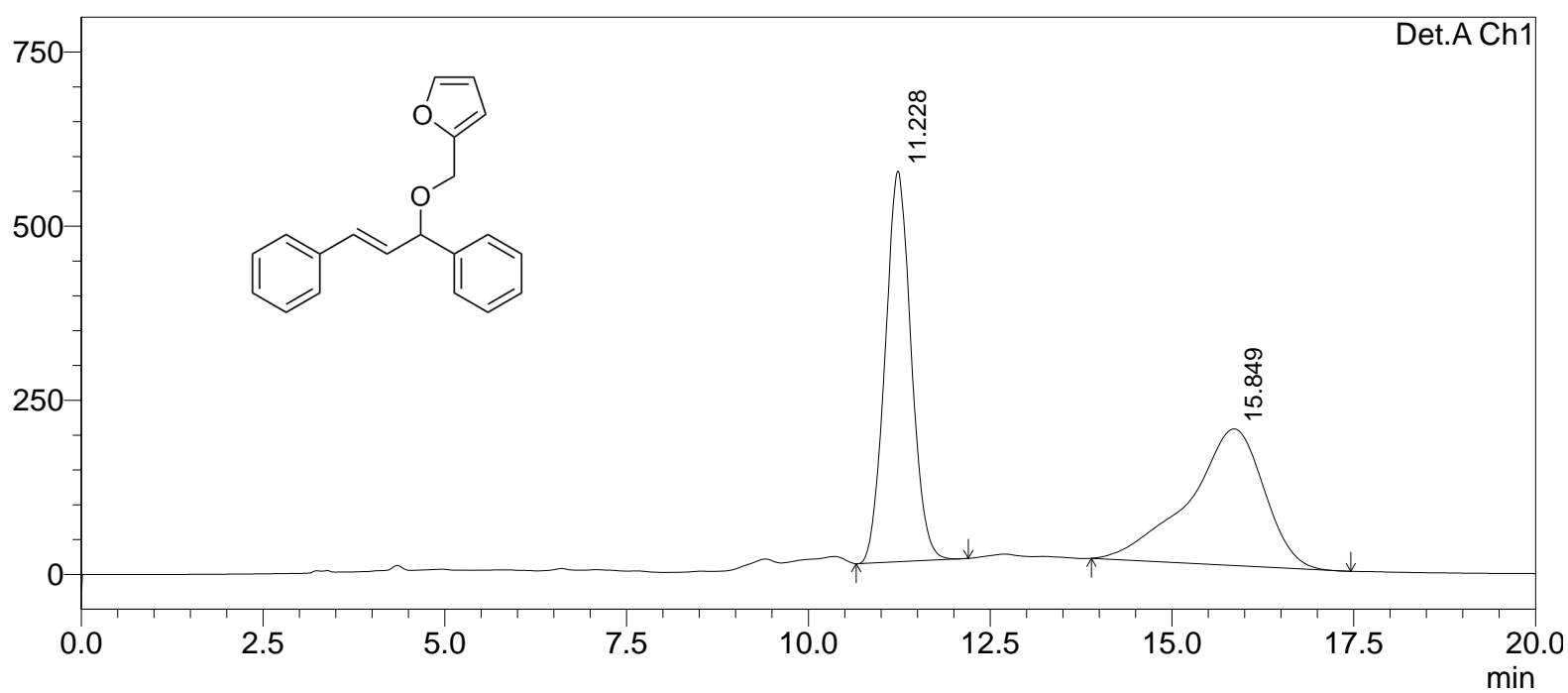

1 Det.A Ch1/254nm

PeakTable

Detector A Ch1 254nm

\begin{tabular}{|r|r|r|r|r|r|}
\hline \multicolumn{1}{|c|}{ Peak\# } & Ret. Time & Area & Height & \multicolumn{1}{|c|}{ Area \% } & \multicolumn{1}{c|}{ Height \% } \\
\hline 1 & 11.228 & 13962560 & 560990 & 49.913 & 74.095 \\
\hline 2 & 15.849 & 14011293 & 196133 & 50.087 & 25.905 \\
\hline Total & & 27973853 & 757124 & 100.000 & 100.000 \\
\hline
\end{tabular}




\section{$====$ Analysis Report $====$}

Acquired by

E:Idatal901ILSSILSS180716-2(OD 10\% 1): Admin :

Sample Name

Sample ID

Vail \# LSS180716-2(OD 10\% 1)

: $1 \mathrm{uL}$

Injection Volume $\quad$ : LSS180716-2(OD 10\% 1)

Data File Name

: $180327 . \mathrm{lcm}$

Method File Name

Batch File Name

Report File Name

Data Acquired

: Default.Icr

2018/7/19 22:13:03

: 2018/7/19 22:42:27

Data Processed

\section{$<$ Chromatogram >}

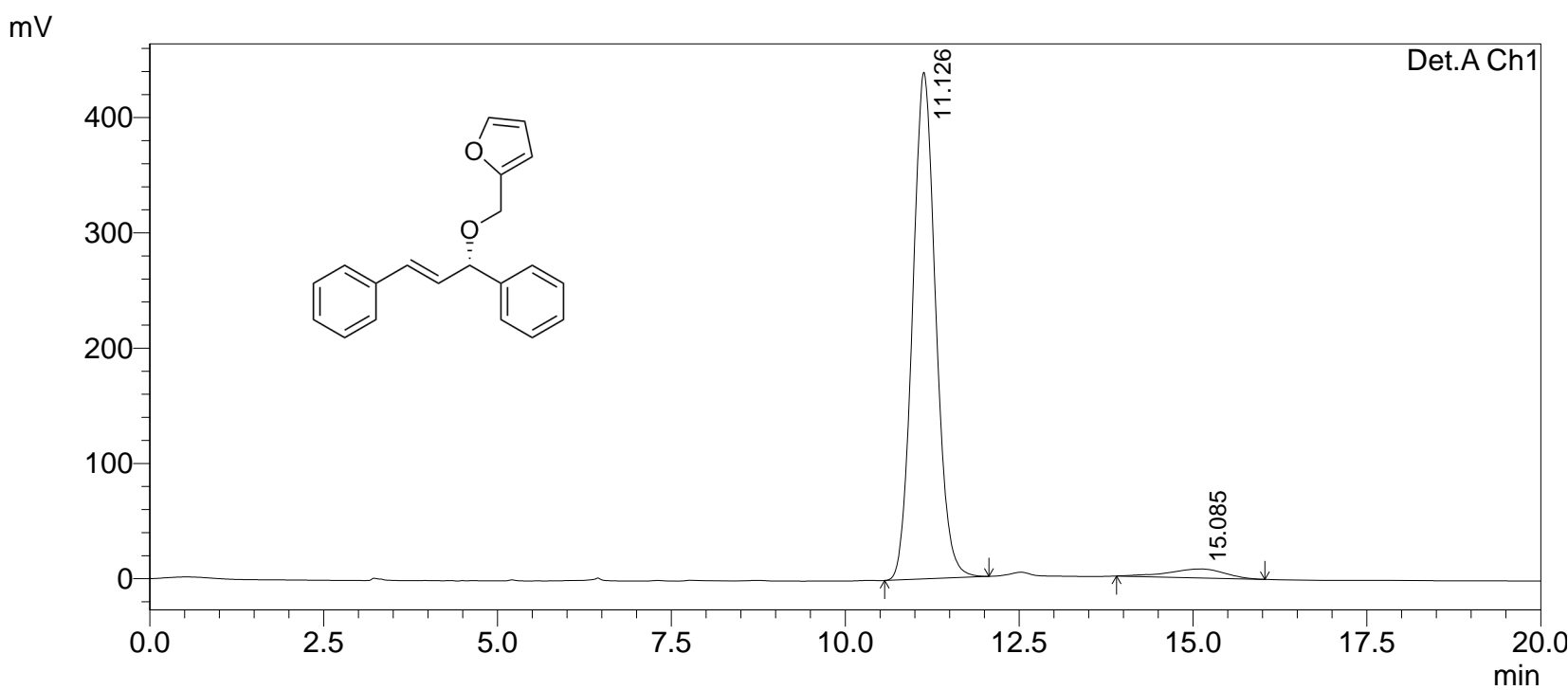

1 Det.A Ch1/254nm

PeakTable

Detector A Ch1 254nm
\begin{tabular}{|r|r|r|r|r|r|}
\hline Peak\# & Ret. Time & Area & \multicolumn{1}{|c|}{ Height } & Area \% & Height $\%$ \\
\hline 1 & 11.126 & 10153372 & 439512 & 95.840 & 98.222 \\
\hline 2 & 15.085 & 440734 & 7954 & 4.160 & 1.778 \\
\hline Total & & 10594106 & 447467 & 100.000 & 100.000 \\
\hline
\end{tabular}




\section{==== Analysis Report ====}

Acquired by Sample Name

E:Idatal901LSSILSS180917-5-XX(OJ 5\% 1.0 254)

: Admin

: LSS180917-5-XX(OJ 5\% 1.0 254)

Sample ID

Vail \#

Injection Volume

Data File Name

$: 1 \mathrm{uL}$

Method File Name

Batch File Name

Report File Name

Data Acquired

: LSS180917-5-XX(OJ 5\% 1.0 254) : 180327.Icm

: Default.lcr

: 2018/9/19 18:29:45

: 2018/9/19 20:27:31

Data Processed

\section{$<$ Chromatogram>}

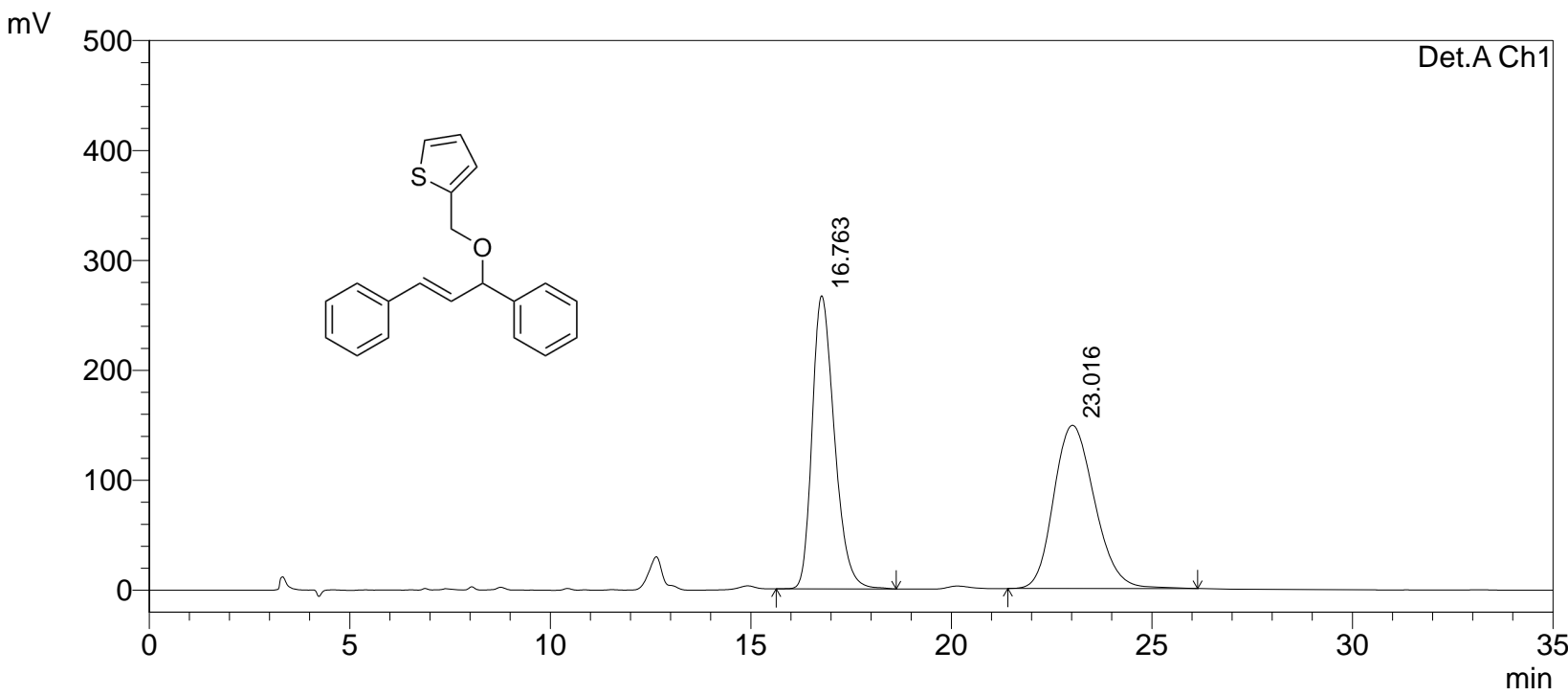

1 Det.A Ch1/254nm

DeakTable
\begin{tabular}{|r|r|r|r|r|r|}
\hline Peak\# & Ret. Time & Area & Height & Area \% & \multicolumn{1}{c|}{ Height \% } \\
\hline 1 & 16.763 & 10278380 & 266698 & 49.422 & 64.233 \\
\hline 2 & 23.016 & 10518609 & 148508 & 50.578 & 35.767 \\
\hline Total & & 20796989 & 415205 & 100.000 & 100.000 \\
\hline
\end{tabular}




\section{==== Analysis Report ====}

Acquired by Sample Name Sample ID

Vail \#

Injection Volume Data File Name Method File Name

Batch File Name

Report File Name

Data Acquired

Data Processed
E:Idatal901ILSSILSS180918-3(OJ 5\% 1 254)saifenjiachun1.Icd

: Admin

: LSS180918-3(OJ 5\% 1 254)saifenjiachun

$: 1 \mathrm{uL}$

: LSS180918-3(OJ 5\% 1 254)saifenjiachun1.Icd

: $180327 . \mathrm{Icm}$

: Default.Icr

2018/9/20 20:13:58

: 2018/9/20 20:46:39

\section{$<$ Chromatogram>}

$\mathrm{mV}$

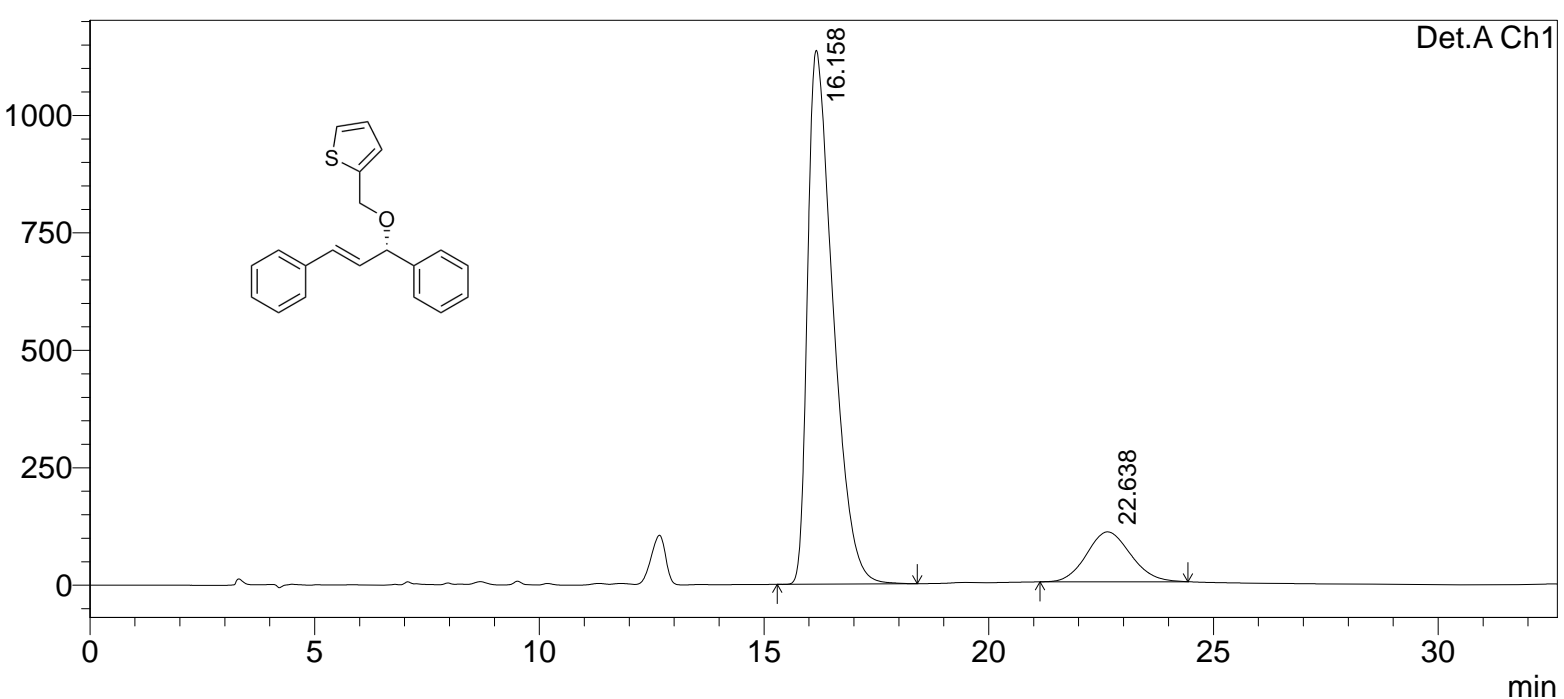

1 Det.A Ch1/254nm

PeakTable

Detector A Ch1 254nm

\begin{tabular}{|r|r|r|r|r|r|}
\hline \multicolumn{1}{|c|}{ Peak\# } & Ret. Time & \multicolumn{1}{|c|}{ Area } & \multicolumn{1}{|c|}{ Height } & \multicolumn{1}{c|}{ Area \% } & \multicolumn{1}{c|}{ Height \% } \\
\hline 1 & 16.158 & 44731748 & 1136641 & 86.024 & 91.454 \\
\hline 2 & 22.638 & 7267528 & 106221 & 13.976 & 8.546 \\
\hline Total & & 51999276 & 1242862 & 100.000 & 100.000 \\
\hline
\end{tabular}




\section{=ニニ= Analysis Report $====$}

Acquired by Sample Name Sample ID

Vail \#

Injection Volume Data File Name Method File Name Batch File Name Report File Name Data Acquired Data Processed
E:Idatal901ILSSILSS180914-3-XX(OD 15\% 0.5 254)

: Admin

: LSS180914-3-XX(OD 15\% 0.5 254)

$:$

$: 1 \mathrm{uL}$

: LSS180914-3-XX(OD 15\% 0.5 254) : 180327.Icm

: Default.lcr

2018/9/17 16:15:37

2018/9/17 16:35:43

\section{$<$ Chromatogram >}

$\mathrm{mV}$

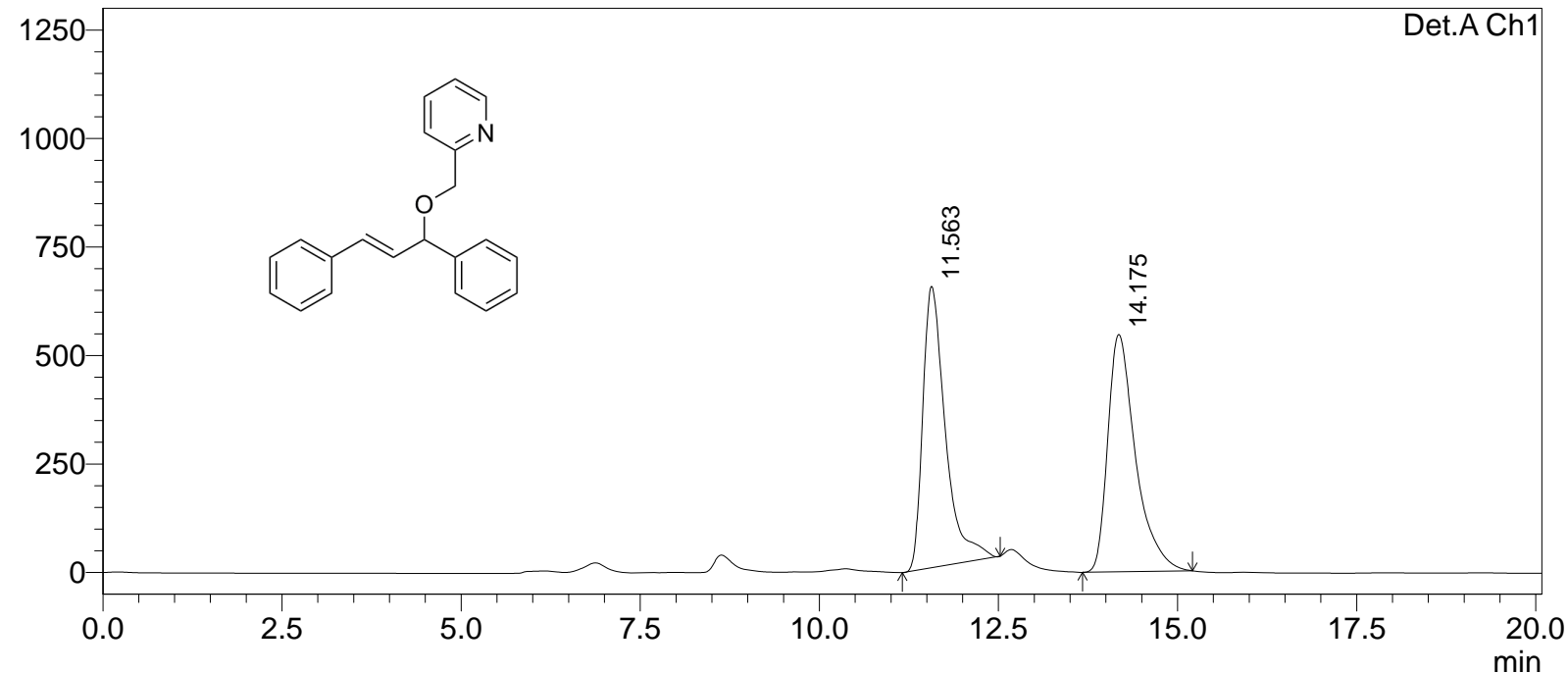

1 Det.A Ch1/254nm

PeakTable

Detector A Ch1 254nm

\begin{tabular}{|r|r|r|r|r|r|}
\hline Peak\# & Ret. Time & \multicolumn{1}{|c|}{ Area } & \multicolumn{1}{|c|}{ Height } & \multicolumn{1}{c|}{ Area \% } & \multicolumn{1}{c|}{ Height \% } \\
\hline 1 & 11.563 & 14258506 & 648652 & 49.150 & 54.253 \\
\hline 2 & 14.175 & 14751647 & 546961 & 50.850 & 45.747 \\
\hline Total & & 29010153 & 1195613 & 100.000 & 100.000 \\
\hline
\end{tabular}




\section{==== Analysis Report ====}

Acquired by Sample Name

E:Idatal901ILSSILSS180914-1(OD 15\% 0.5 254)

: Admin

: LSS180914-1(OD 15\% 0.5 254)

Sample ID

Vail \#

Injection Volume

Data File Name

$1 \mathrm{uL}$

Method File Name

Batch File Name

Report File Name

Data Acquired

: LSS180914-1(OD 15\% 0.5 254) : 180327.lcm

: Default.Icr

: 2018/9/17 17:32:17

: 2018/9/17 18:01:14

Data Processed

\section{$<$ Chromatogram >}

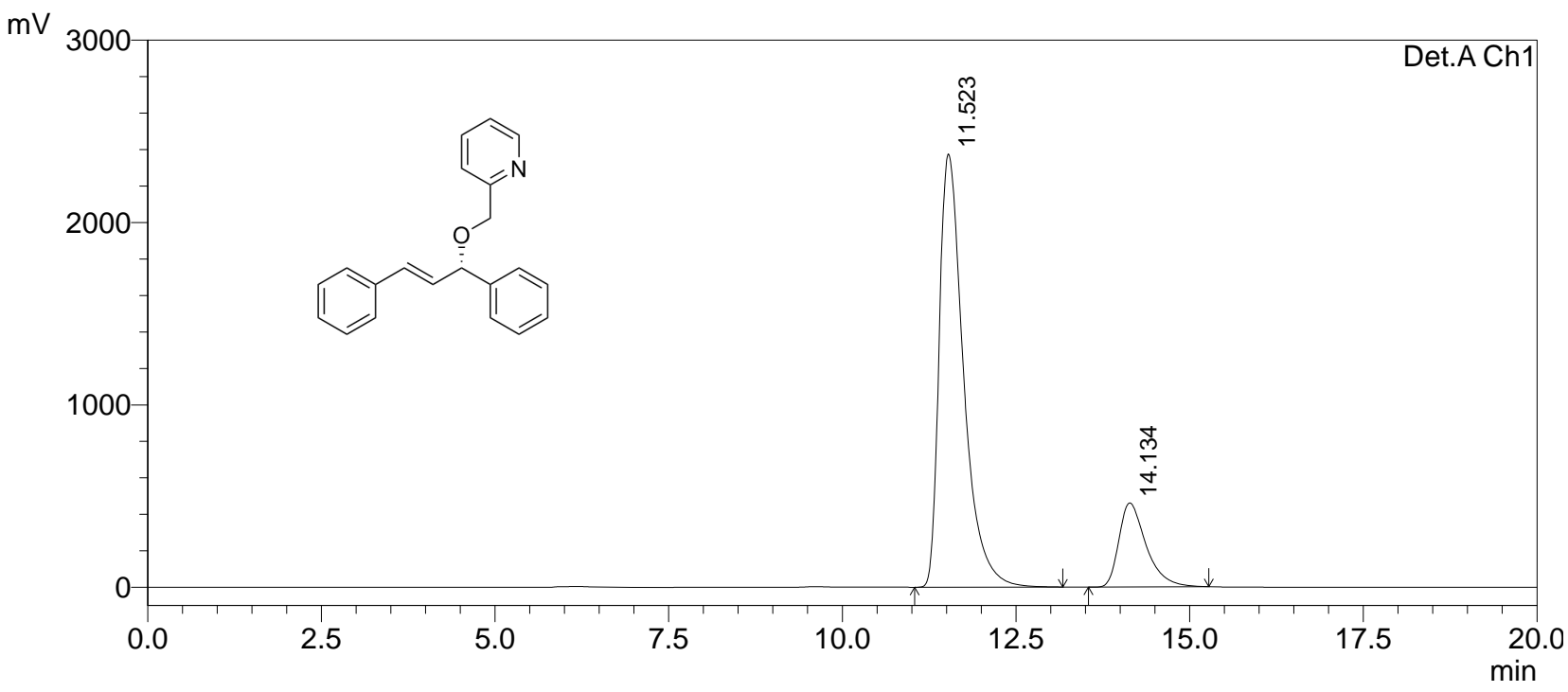

1 Det.A Ch1/254nm

PeakTable

Detector A Ch1 254nm

\begin{tabular}{|r|r|r|r|r|r|}
\hline \multicolumn{1}{|c|}{ Peak\# } & Ret. Time & \multicolumn{1}{|c|}{ Area } & \multicolumn{1}{|c|}{ Height } & Area \% & \multicolumn{1}{c|}{ Height \% } \\
\hline 1 & 11.523 & 57881011 & 2375298 & 82.164 & 83.747 \\
\hline 2 & 14.134 & 12564824 & 460973 & 17.836 & 16.253 \\
\hline Total & & 70445836 & 2836271 & 100.000 & 100.000 \\
\hline
\end{tabular}




\section{==== Analysis Report ====}

Acquired by Sample Name Sample ID

Vail \#

Injection Volume Data File Name Method File Name Batch File Name

Report File Name Data Acquired

Data Processed
E:Idatal901ILSSILSS180917-4-XX(AD 1\% 1254$)$

: Admin

: LSS180917-4-XX(AD 1\% 1 254)

:

$: 1 \mathrm{uL}$

: LSS180917-4-XX(AD 1\% 1 254) : 180327.Icm

: Default.lcr

: 2018/9/19 9:48:08

: 2018/9/19 10:14:10

\section{$<$ Chromatogram>}

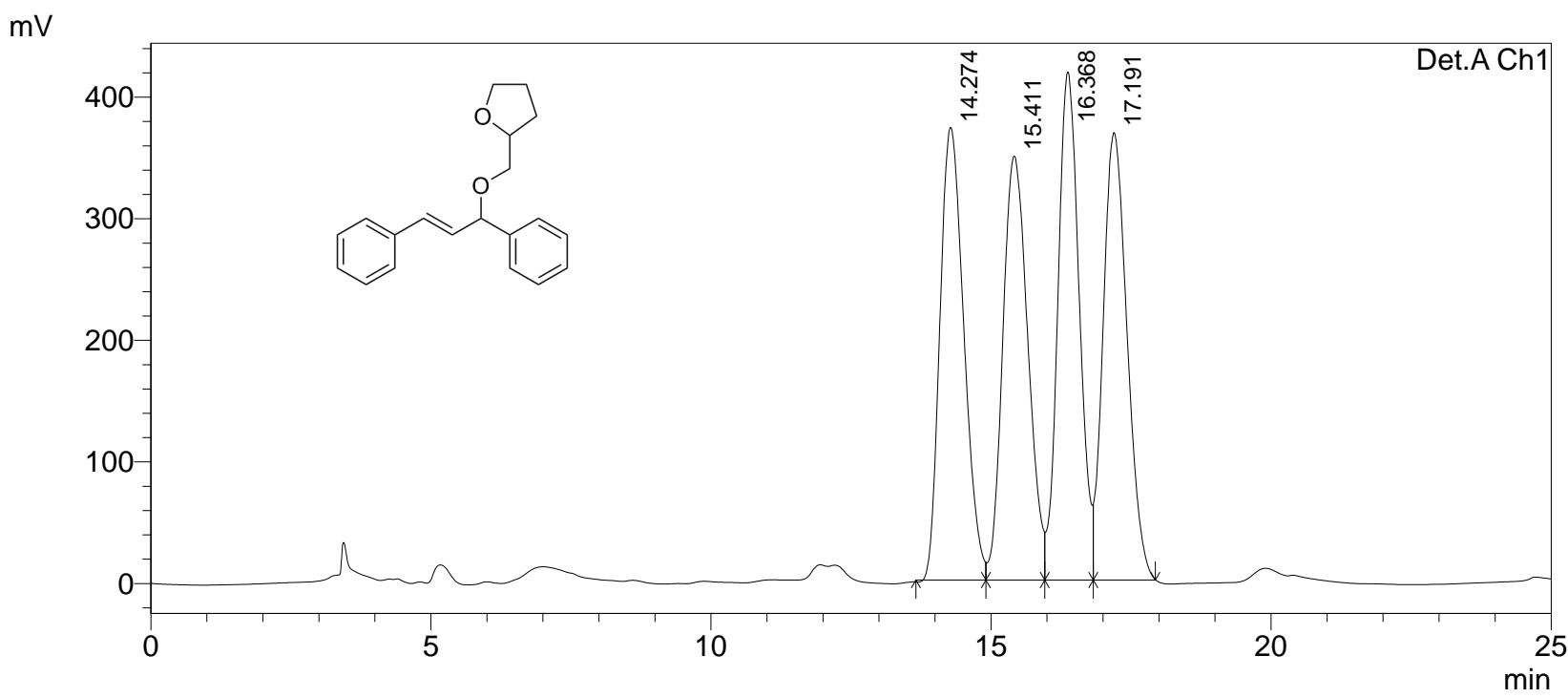

1 Det.A Ch1/254nm

Detector A Ch1 254nm

PeakTable

\begin{tabular}{|r|r|r|r|r|r|}
\hline \multicolumn{1}{|c|}{ Peak\# } & Ret. Time & Area & Height & \multicolumn{1}{c|}{ Area \% } & \multicolumn{1}{c|}{ Height \% } \\
\hline 1 & 14.274 & 11037418 & 372243 & 25.062 & 24.704 \\
\hline 2 & 15.411 & 10896156 & 348689 & 24.742 & 23.141 \\
\hline 3 & 16.368 & 11154278 & 417897 & 25.328 & 27.734 \\
\hline 4 & 17.191 & 10951987 & 367997 & 24.868 & 24.422 \\
\hline Total & & 44039839 & 1506826 & 100.000 & 100.000 \\
\hline
\end{tabular}




\section{$====$ Analysis Report $====$}

Acquired by Sample Name

E:Idatal901ILSSILSS180917-2(AD 1\% 0.5 254)

: Admin

: LSS180917-2(AD 1\% 1 254)

Sample ID

Vail \#

Injection Volume

: $1 \mathrm{uL}$

Data File Name

Method File Name

Batch File Name

Report File Name

Data Acquired

: LSS180917-2(AD 1\% 0.5 254) : 180327.Icm

: Default.lcr

: 2018/9/19 13:28:43

: 2018/9/19 13:50:19

Data Processed

\section{$<$ Chromatogram >}

$\mathrm{mV}$

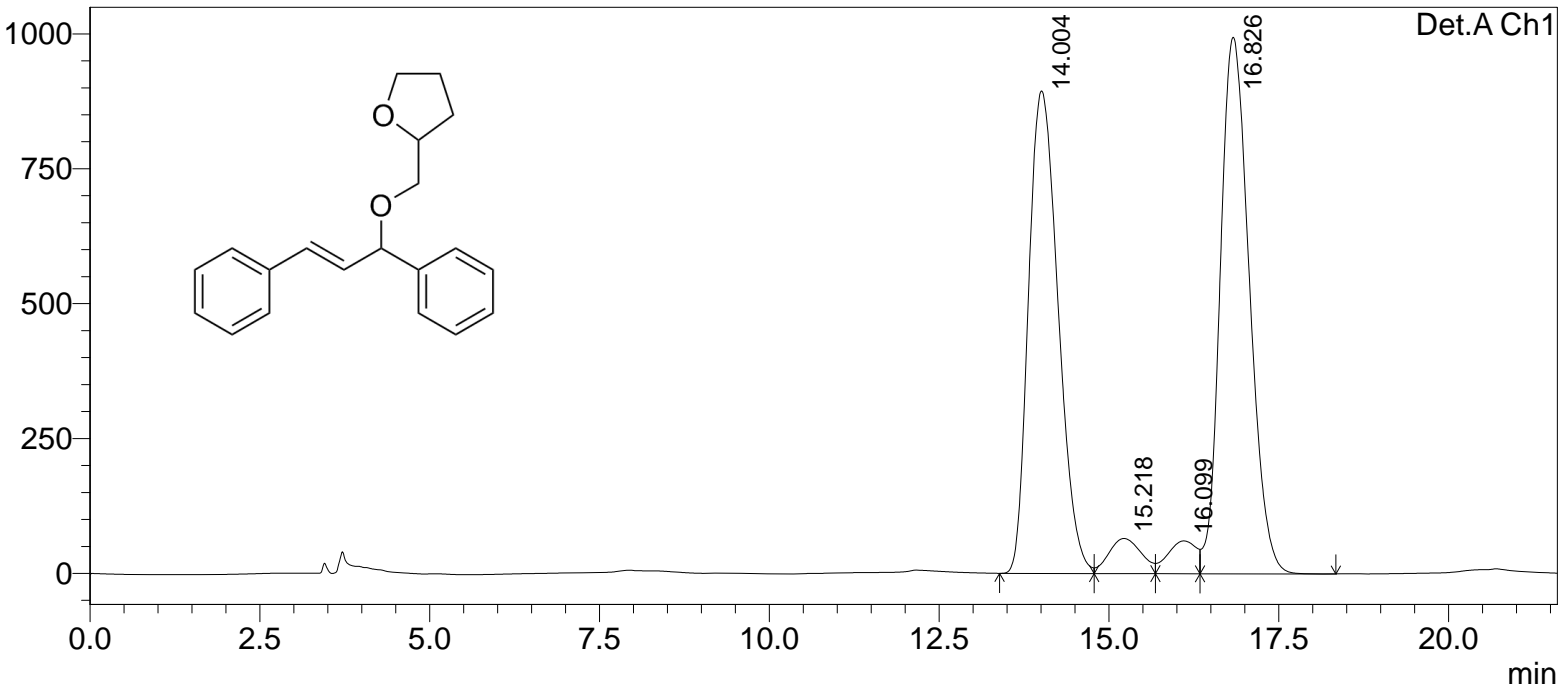

1 Det.A Ch1/254nm

PeakTable

Detector A Ch1 254nm

\begin{tabular}{|r|r|r|r|r|r|}
\hline \multicolumn{1}{|c|}{ Peak\# } & Ret. Time & \multicolumn{1}{c|}{ Area } & \multicolumn{1}{c|}{ Height } & Area \% & \multicolumn{1}{c|}{ Height \% } \\
\hline 1 & 14.004 & 27150105 & 894443 & 44.645 & 44.390 \\
\hline 2 & 15.218 & 2173664 & 65125 & 3.574 & 3.232 \\
\hline 3 & 16.099 & 1789692 & 60893 & 2.943 & 3.022 \\
\hline 4 & 16.826 & 29699665 & 994489 & 48.838 & 49.356 \\
\hline Total & & 60813125 & 2014950 & 100.000 & 100.000 \\
\hline
\end{tabular}




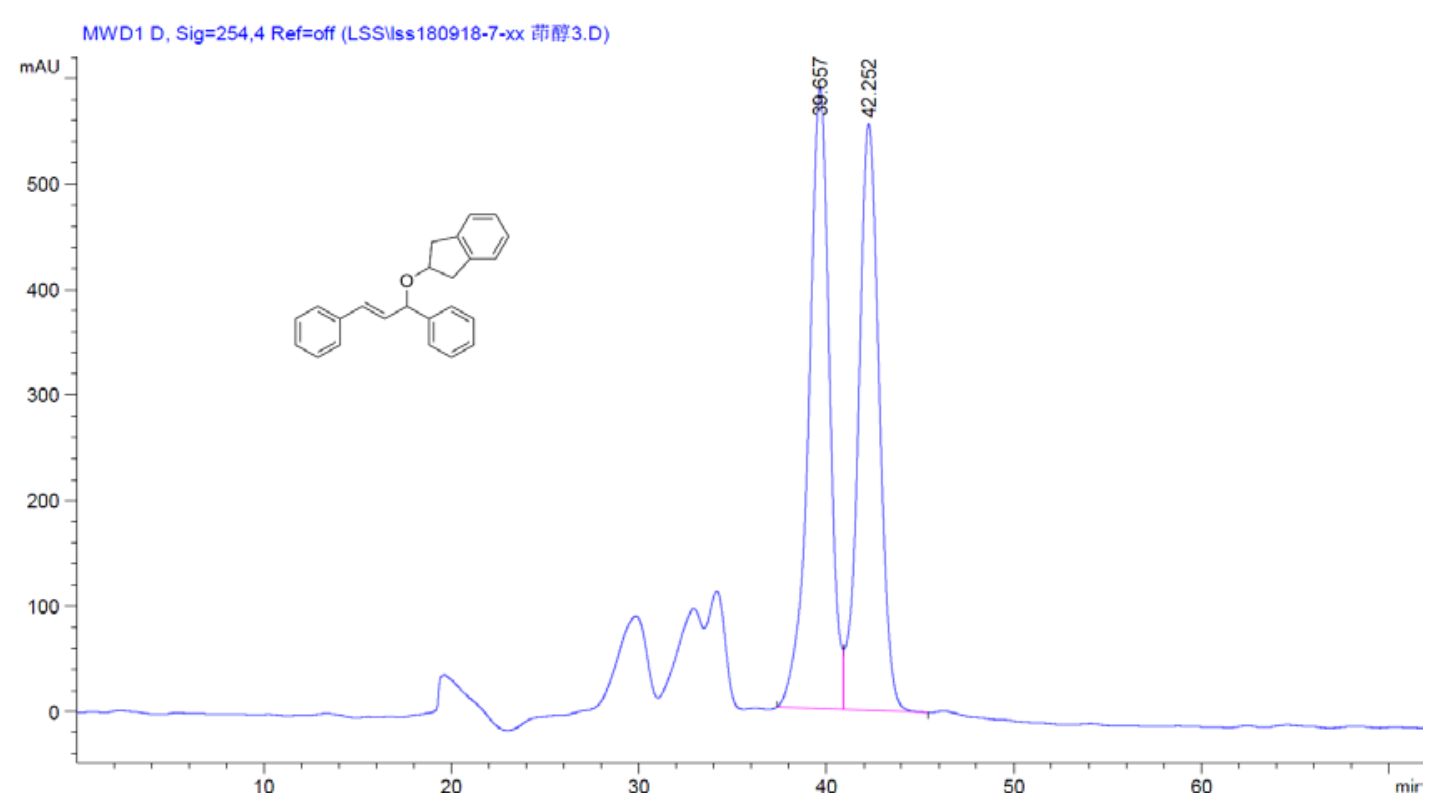

峰 保留时间 类型 峰宽 峰面积峰高峰面积

\begin{tabular}{cccccc}
$\#$ & [min] & [min] & {$[\mathrm{mAU} * \mathrm{~s}]$} & {$[\mathrm{mAU}]$} & $\%$ \\
\hline 1 & 39.657 BV & 1.1598 & $4.53568 \mathrm{e} 4$ & 588.07245 & 51.5222 \\
2 & $42.252 \mathrm{VB}$ & 1.1639 & $4.26766 \mathrm{e} 4$ & 555.76849 & 48.4778
\end{tabular}

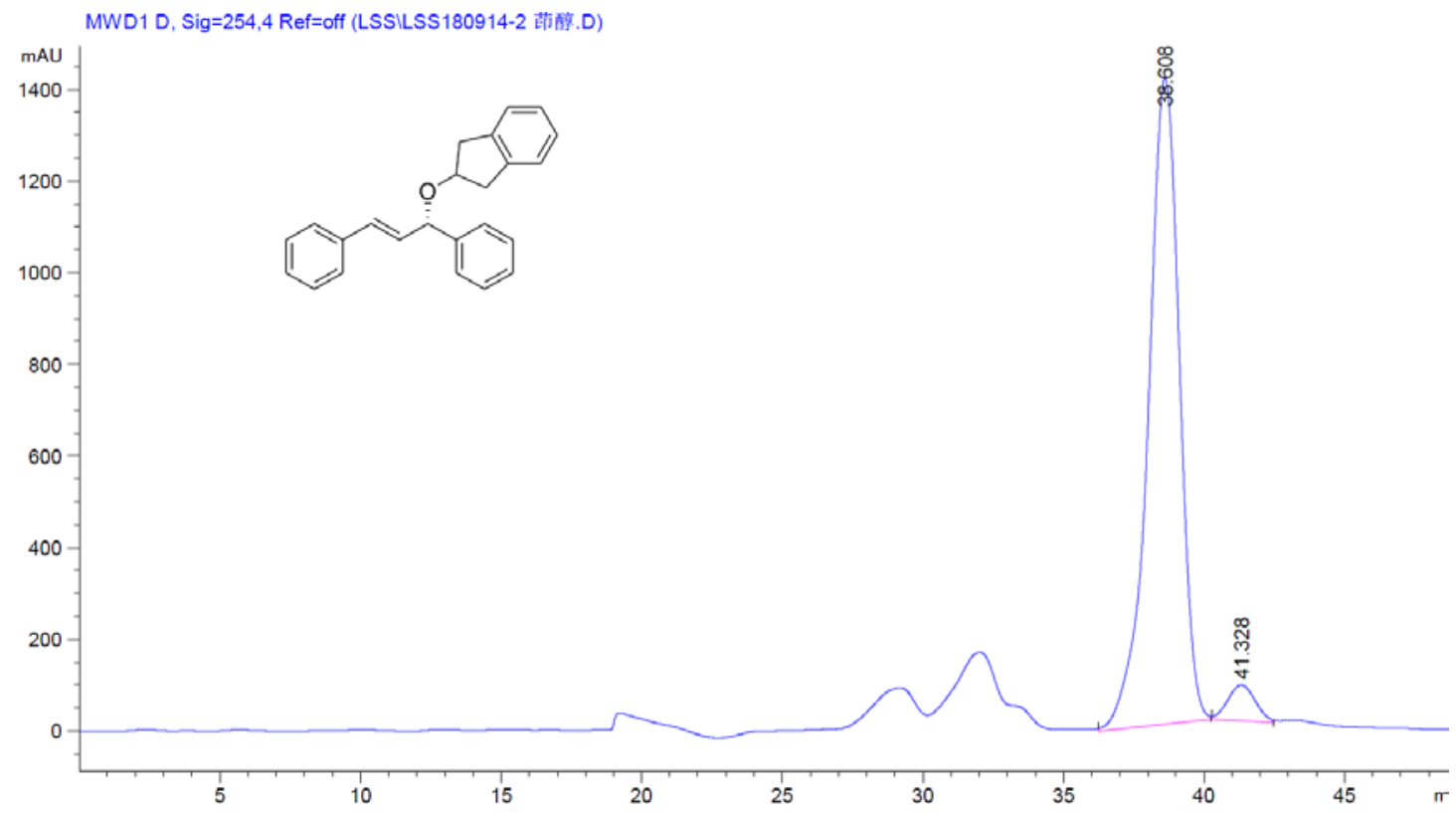

峰保留时间 类型峰宽峰面积峰高峰面积

\begin{tabular}{cccccr}
$\#$ & {$[\mathrm{~min}]$} & {$[\mathrm{min}]$} & {$[\mathrm{mAU} * \mathrm{~s}]$} & \multicolumn{1}{c}{$[\mathrm{mAU}]$} & \multicolumn{1}{c}{$\%$} \\
\hline 1 & $38.608 \mathrm{MM}$ & 1.2805 & $1.08390 \mathrm{e} 5$ & 1410.76782 & 95.3264 \\
2 & $41.328 \mathrm{MM}$ & 1.1332 & 5314.08936 & 78.15599 & 4.6736
\end{tabular}




\section{=ニ== Analysis Report $====$}

Acquired by Sample Name

Sample ID

Vail \#

E:Idatal901ILSSILSS180911-6(OJ 3\% 1 254)

Injection Volume

Data File Name

Method File Name

Batch File Name

Report File Name

Data Acquired : LSS180911-6(OJ 3\% 1 254)

: $1 \mathrm{uL}$

: LSS180911-6(OJ 3\% 1 254)

: $180327 . \mathrm{Icm}$

- Default.Icr

: 2018/9/14 20:15:10

: 2018/9/14 21:02:06

Data Processed

\section{$<$ Chromatogram $>$}

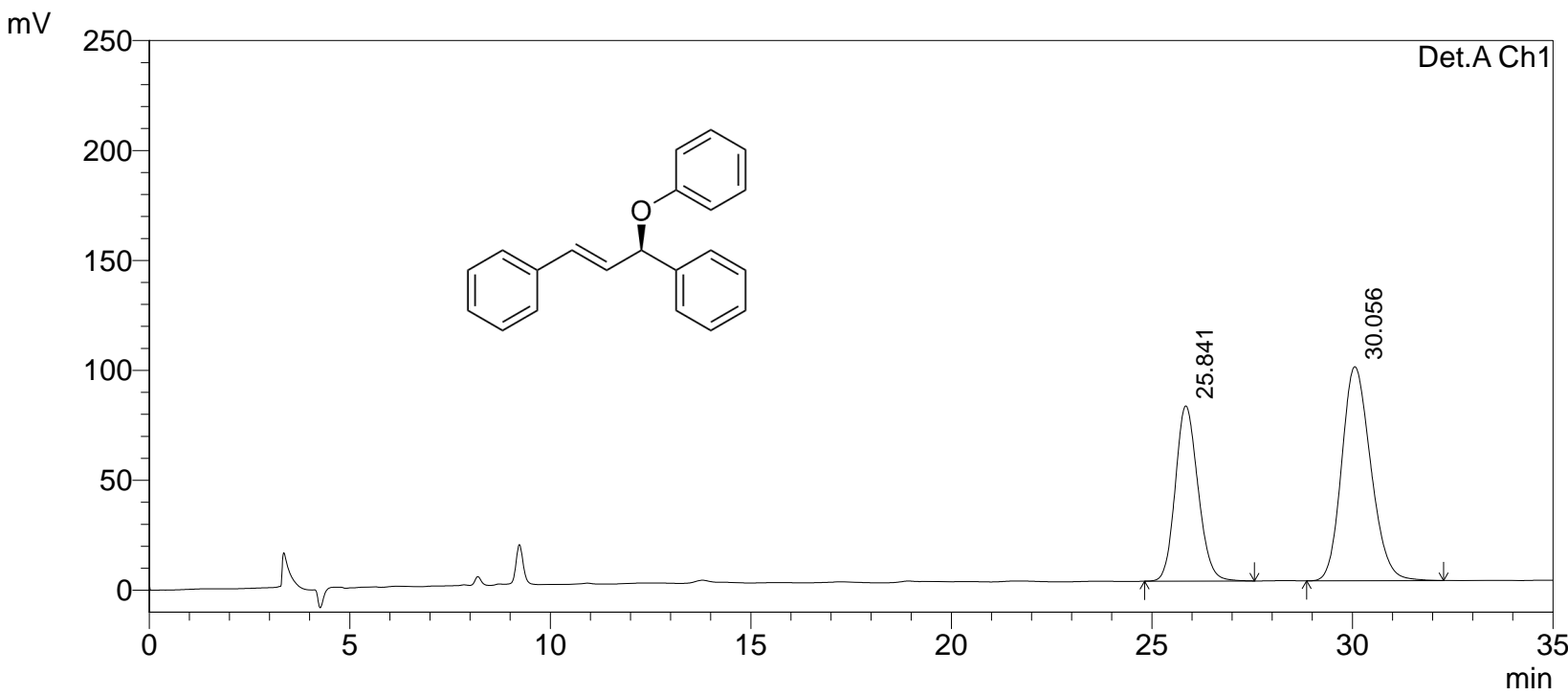

1 Det.A Ch1/254nm

PeakTable

Detector A Ch1 254nm

\begin{tabular}{|r|r|r|r|r|r|}
\hline \multicolumn{1}{|c|}{ Peak\# } & Ret. Time & \multicolumn{1}{c|}{ Area } & Height & \multicolumn{1}{c|}{ Area \% } & \multicolumn{1}{c|}{ Height \% } \\
\hline 1 & 25.841 & 3131727 & 79718 & 38.899 & 45.003 \\
\hline 2 & 30.056 & 4919165 & 97422 & 61.101 & 54.997 \\
\hline Total & & 8050891 & 177140 & 100.000 & 100.000 \\
\hline
\end{tabular}




\section{$====$ Analysis Report $====$}

Acquired by

E:Idatal901ILSSILSS180828-4-XX(AD 0.3\% 0.3 254)3.Icd : Admin

Sample Name

Sample ID

Vail \# : LSS180828-4-XX(AD 0.3\% 0.3254$)$

Injection Volume $\quad:$ LSS180828-4-XX(AD 0.3\% 0.3 254)3.Icd

Data File Name

Method File Name

Batch File Name

Report File Name

Data Acquired

: $180327 . \mathrm{lcm}$

: Default.Icr

: 2018/9/3 15:20:10

: 2018/9/3 15:50:12

Data Processed

\section{$<$ Chromatogram $>$}

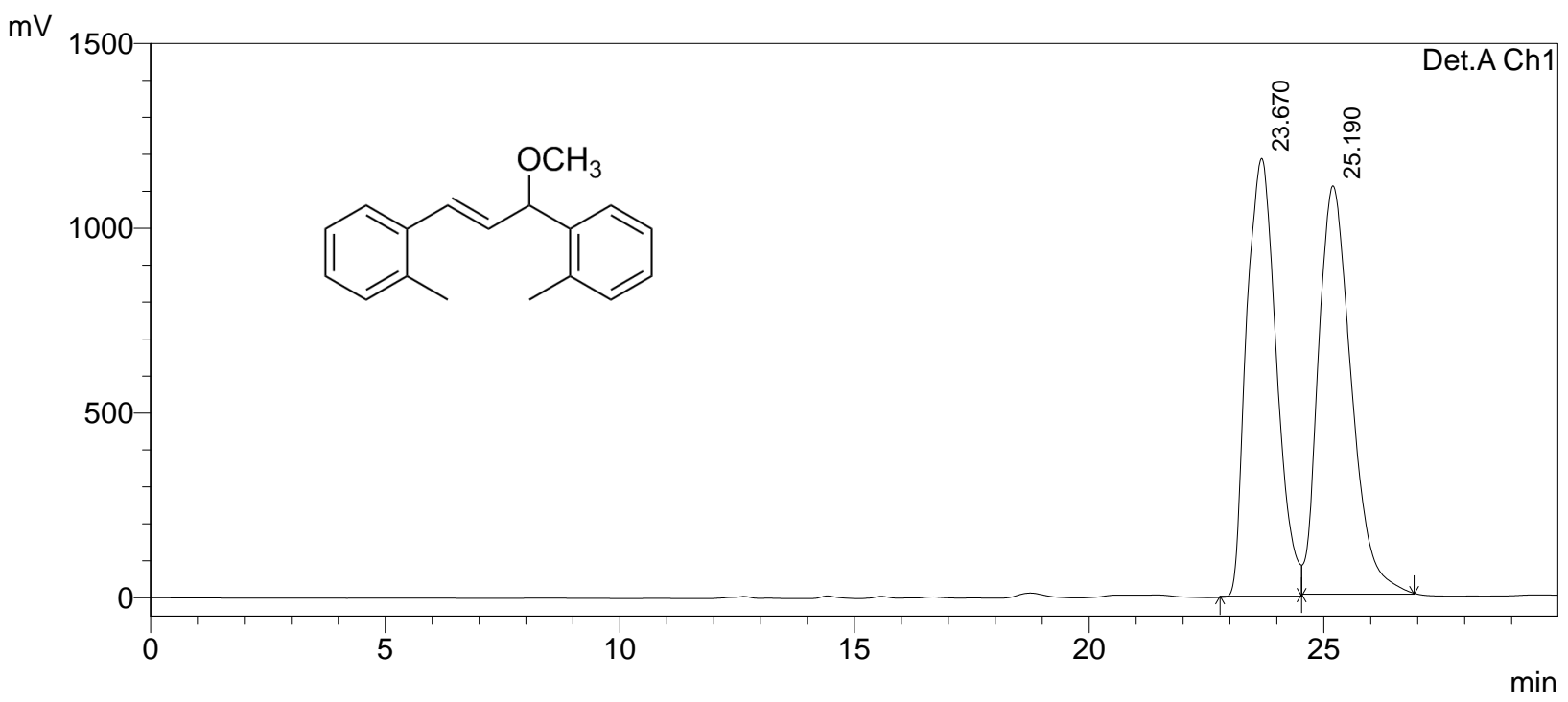

1 Det.A Ch1/254nm

PeakTable

Detector A Ch1 254nm
\begin{tabular}{|r|r|r|r|r|r|}
\hline Peak\# & Ret. Time & \multicolumn{1}{|c|}{ Area } & Height & Area \% & \multicolumn{1}{|c|}{ Height \% } \\
\hline 1 & 23.670 & 52302295 & 1184753 & 49.187 & 51.731 \\
\hline 2 & 25.190 & 54030803 & 1105474 & 50.813 & 48.269 \\
\hline Total & & 106333097 & 2290227 & 100.000 & 100.000 \\
\hline
\end{tabular}




\section{=ニニ= Analysis Report $====$}

Acquired by

E:Idatal901ILSSILSS180830-1-o(AD 0.3\% 0.3 254)2.Icd : Admin

Sample Name

Sample ID

Vail \# : LSS180830-1-o(AD 0.3\% 0.3254$)$

Injection Volume $\quad$ : LSS180830-1-o(AD 0.3\% 0.3 254)2.Icd

Data File Name

Method File Name

Batch File Name

Report File Name

Data Acquired

180327.Icm

: Default.Icr

: 2018/9/2 22:21:29

: 2018/9/2 22:53:49

Data Processed

\section{$<$ Chromatogram $>$}

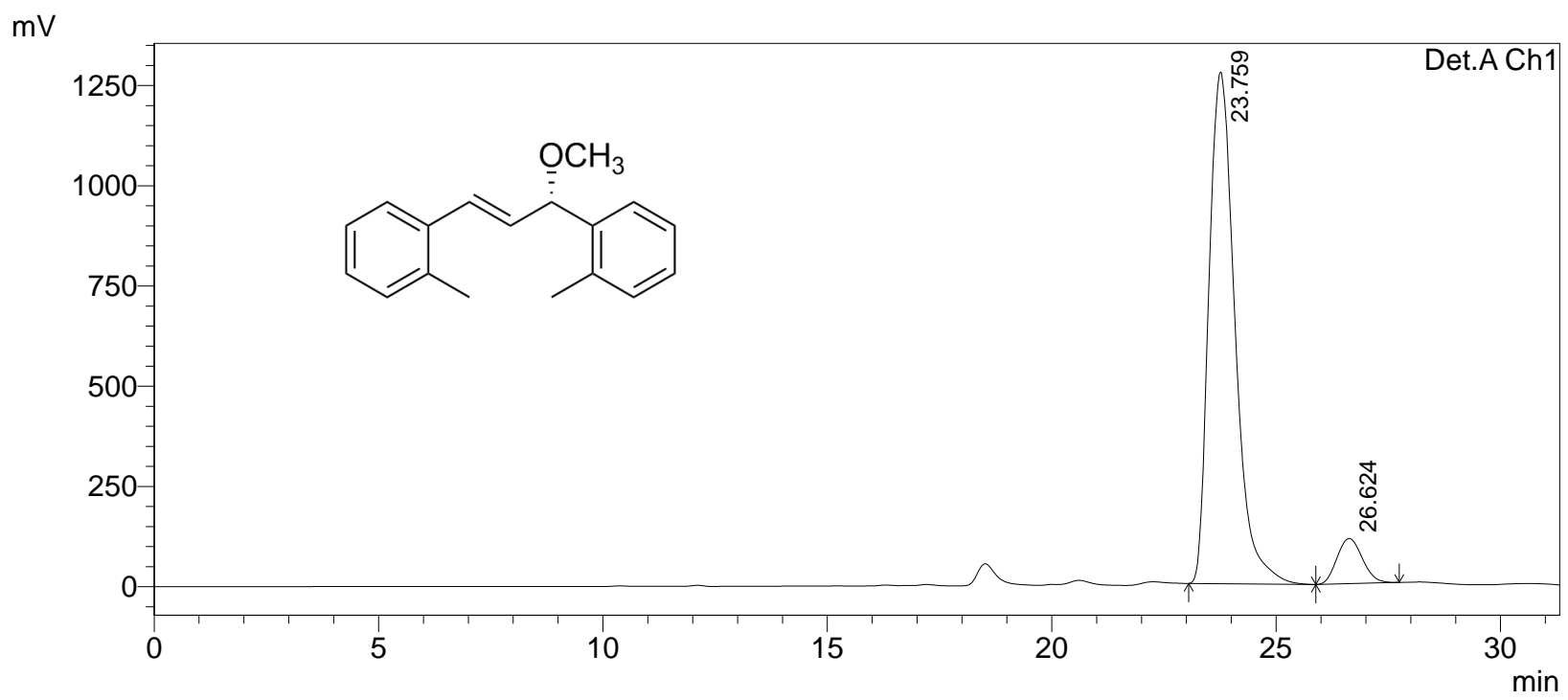

1 Det.A Ch1/254nm

Detector A Ch1 254nm

PeakTable

\begin{tabular}{|r|r|r|r|r|r|}
\hline \multicolumn{1}{|c|}{ Peak\# } & \multicolumn{1}{|c|}{ Ret. Time } & \multicolumn{1}{c|}{ Area } & \multicolumn{1}{c|}{ Height } & Area \% & \multicolumn{1}{c|}{ Height \% } \\
\hline 1 & 23.759 & 51448368 & 1276117 & 92.074 & 91.893 \\
\hline 2 & 26.624 & 4428825 & 112582 & 7.926 & 8.107 \\
\hline Total & & 55877193 & 1388699 & 100.000 & 100.000 \\
\hline
\end{tabular}




\section{$== \pm=$ Analysis Report $====$}

Acquired by

E:Idatal901ILSSILSS180828-2-m-XX(OJ 0.5\% 1 254)6.Icd : Admin

Sample Name

Sample ID

Vail \# : LSS180828-2-m-XX(OJ 0.5\% 1 254)

Injection Volume $\quad$ : LSS180828-2-m-XX(OJ 0.5\% 1254$) 6$. Icd

Data File Name

Method File Name

Batch File Name

Report File Name

Data Acquired

$180327 . \mathrm{Icm}$

Default.Icr

: 2018/9/1 17:32:42

2018/9/1 18:17:56

Data Processed

\section{$<$ Chromatogram >}

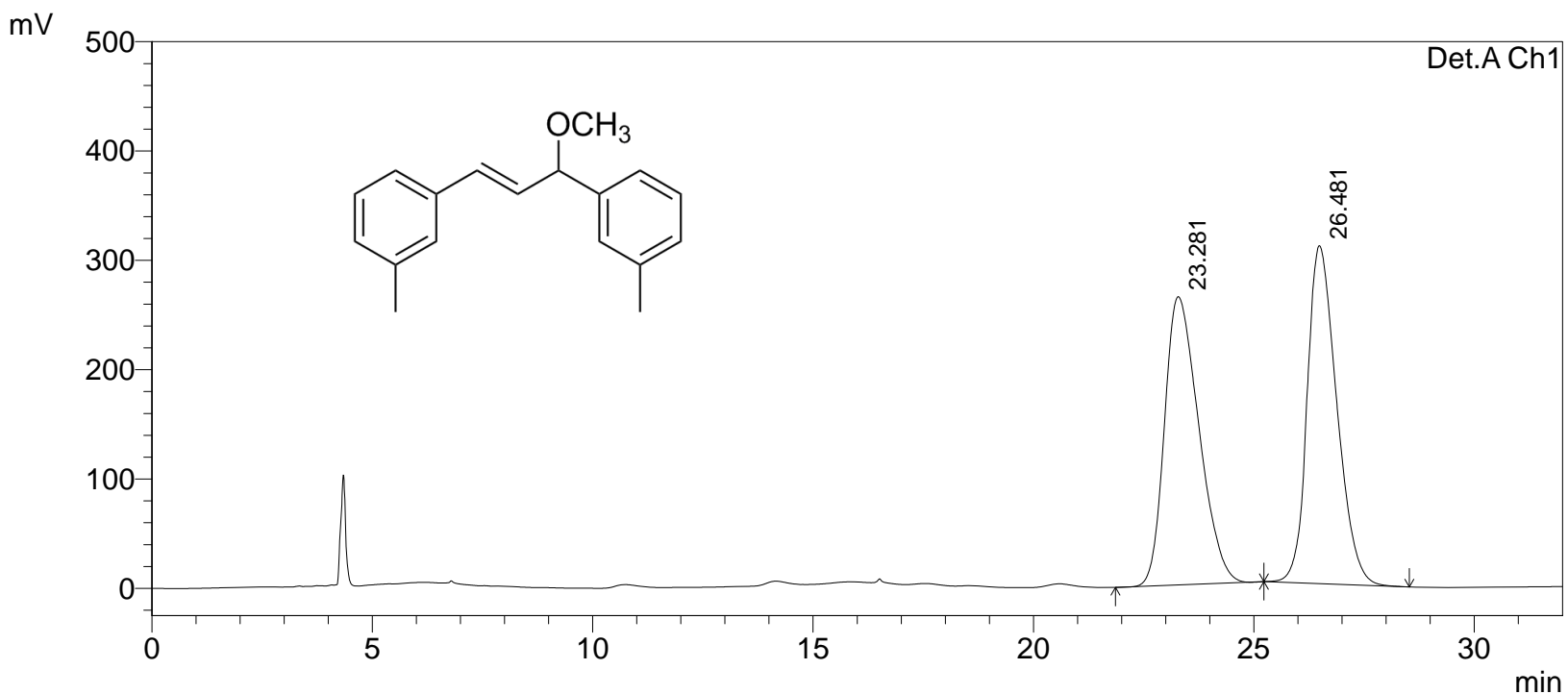

1 Det.A Ch1/254nm

PeakTable

Detector A Ch1 254nm

\begin{tabular}{|r|r|r|r|r|r|}
\hline \multicolumn{1}{|c|}{ Peak\# } & Ret. Time & Area & \multicolumn{1}{|c|}{ Height } & \multicolumn{1}{|c|}{ Area \% } & \multicolumn{1}{|c|}{ Height \% } \\
\hline 1 & 23.281 & 14232096 & 263653 & 49.645 & 46.026 \\
\hline 2 & 26.481 & 14435520 & 309185 & 50.355 & 53.974 \\
\hline Total & & 28667616 & 572838 & 100.000 & 100.000 \\
\hline
\end{tabular}




\section{$====$ Analysis Report $====$}

Acquired by

Sample Name

Sample ID

Vail \#

Injection Volume

Data File Name

Method File Name

Batch File Name

Report File Name

Data Acquired

Data Processed

\section{E:Idatal901ILSSILSS180830-2-m(OJ 0.5\% 1 254)8.Icd : Admin} : LSS180830-2-m(OJ 0.5\% 1 254)

: $1 \mathrm{uL}$

: LSS180830-2-m(OJ 0.5\% 1 254)8.Icd

: $180327 . \mathrm{lcm}$

: Default.lcr

: 2018/9/1 18:54:18

2018/9/1 19:29:05

\section{$<$ Chromatogram >}

$\mathrm{mV}$

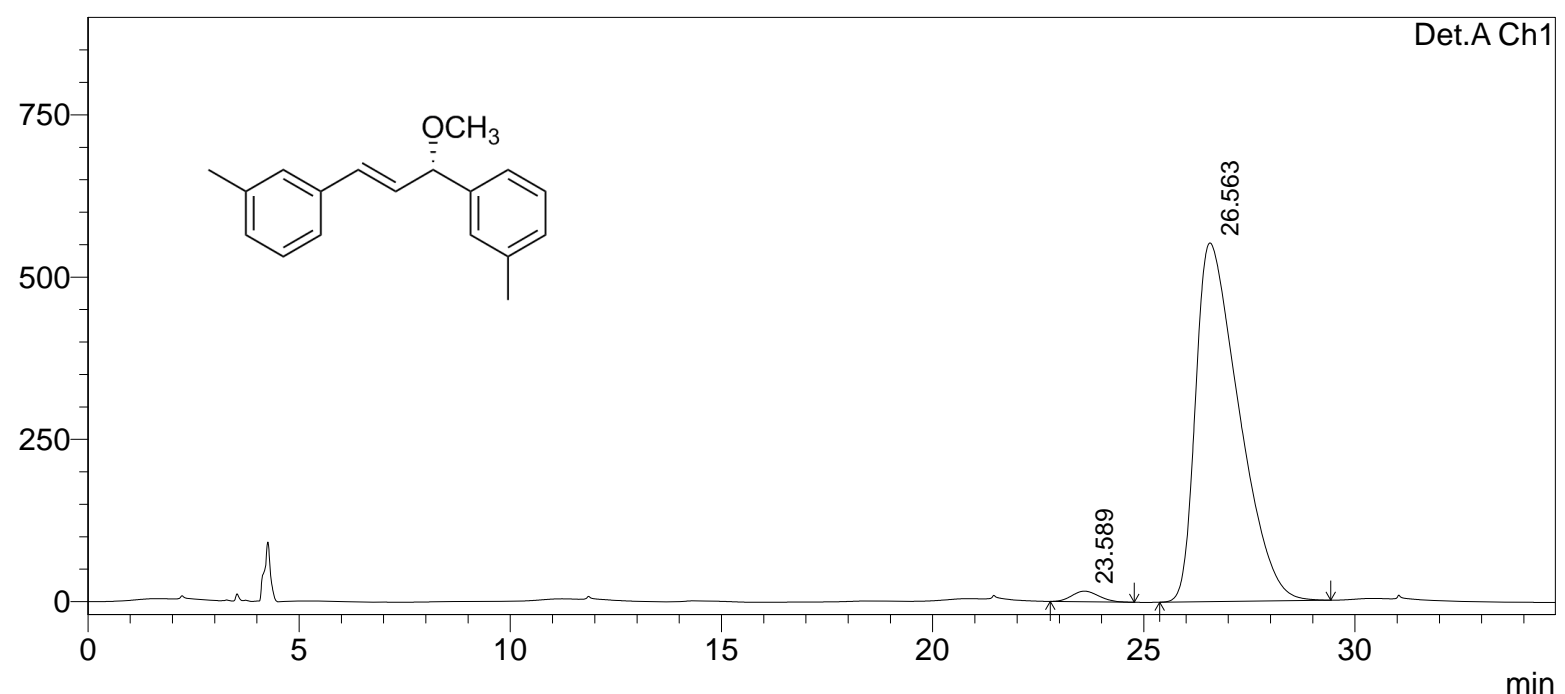

1 Det.A Ch1/254nm

Detector A Ch1 254nm
\begin{tabular}{|r|r|r|r|r|r|}
\hline Peak\# & Ret. Time & Area & Height & Area \% & \multicolumn{1}{|c|}{ Height \% } \\
\hline 1 & 23.589 & 726968 & 16323 & 1.817 & 2.869 \\
\hline 2 & 26.563 & 39285706 & 552634 & 98.183 & 97.131 \\
\hline Total & & 40012674 & 568957 & 100.000 & 100.000 \\
\hline
\end{tabular}




\section{=ニニ= Analysis Report $====$}

Acquired by

E:Idatal901ILSSILSS180820-2-XX(OJ 0.3\% 254 2)11.Icd : Admin

Sample Name

Sample ID

Vail \# : LSS180820-2-XX(OJ 0.3\% 254 2)

Injection Volume $\quad$ : LSS180820-2-XX(OJ 0.3\% 254 2)11.Icd

Data File Name

Method File Name

Batch File Name

Report File Name

Data Acquired

: $180327 . \mathrm{lcm}$

: Default.Icr

: 2018/8/25 21:19:49

: 2018/8/25 21:50:00

Data Processed

\section{$<$ Chromatogram>}

$\mathrm{mV}$

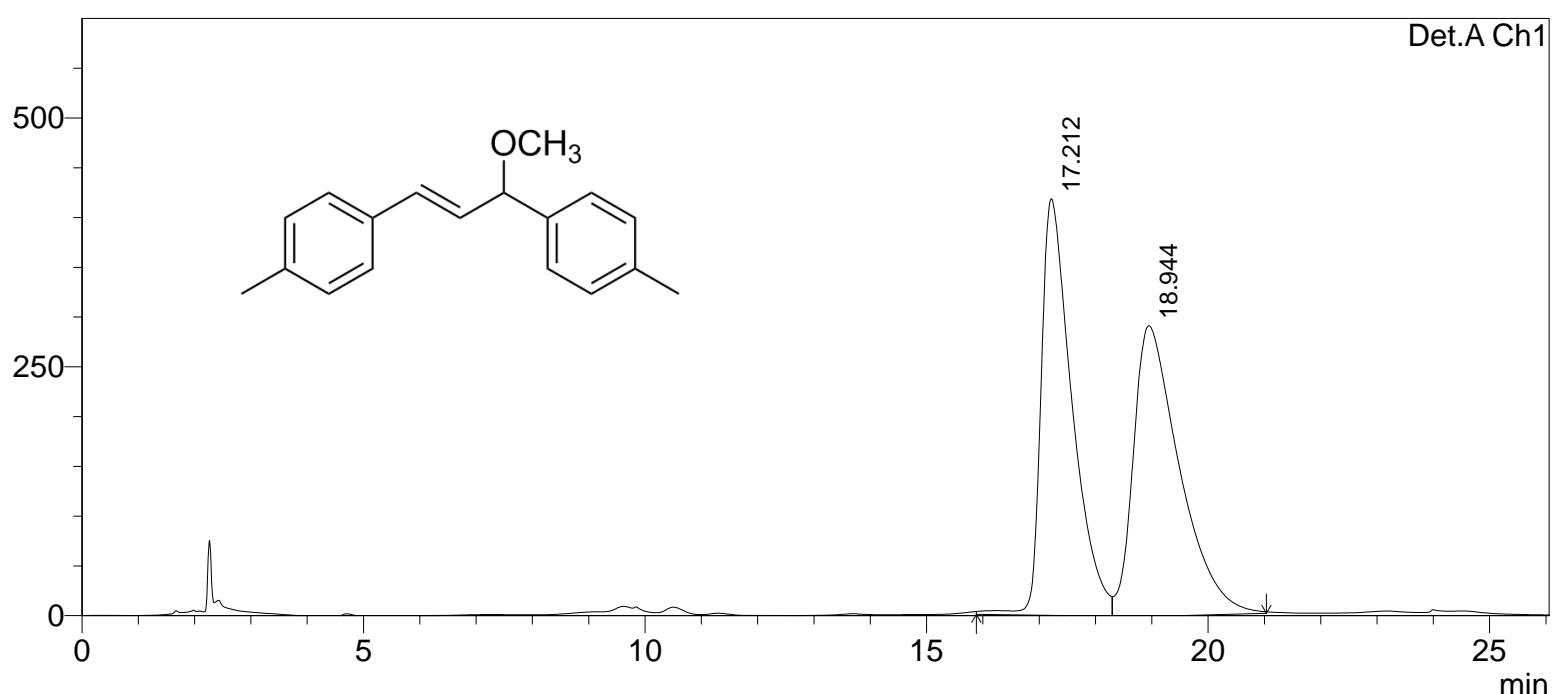

1 Det.A Ch1/254nm

PeakTable

Detector A Ch1 254nm

\begin{tabular}{|r|r|r|r|r|r|}
\hline \multicolumn{1}{|c|}{ Peak\# } & Ret. Time & Area & Height & \multicolumn{1}{|c|}{ Area \% } & \multicolumn{1}{|c|}{ Height \% } \\
\hline 1 & 17.212 & 16096311 & 418780 & 49.149 & 58.904 \\
\hline 2 & 18.944 & 16653596 & 292178 & 50.851 & 41.096 \\
\hline Total & & 32749908 & 710958 & 100.000 & 100.000 \\
\hline
\end{tabular}




\section{===+ Analysis Report ====}

Acquired by

E:Idatal901LSSILSS180821-2(OJ 0.3\% 254 2)12.Icd : Admin

Sample Name

Sample ID

Vail \# : LSS180821-2(OJ 0.3\% 254 2)

: $1 \mathrm{uL}$

Injection Volume $\quad$ : LSS180821-2(OJ 0.3\% 254 2)12.Icd

Data File Name : : 180327.Icm

Method File Name

Batch File Name

Report File Name

Data Acquired

:

: Default.Icr

: 2018/8/25 21:47:50

: 2018/8/25 22:10:16

Data Processed

\section{$<$ Chromatogram $>$}

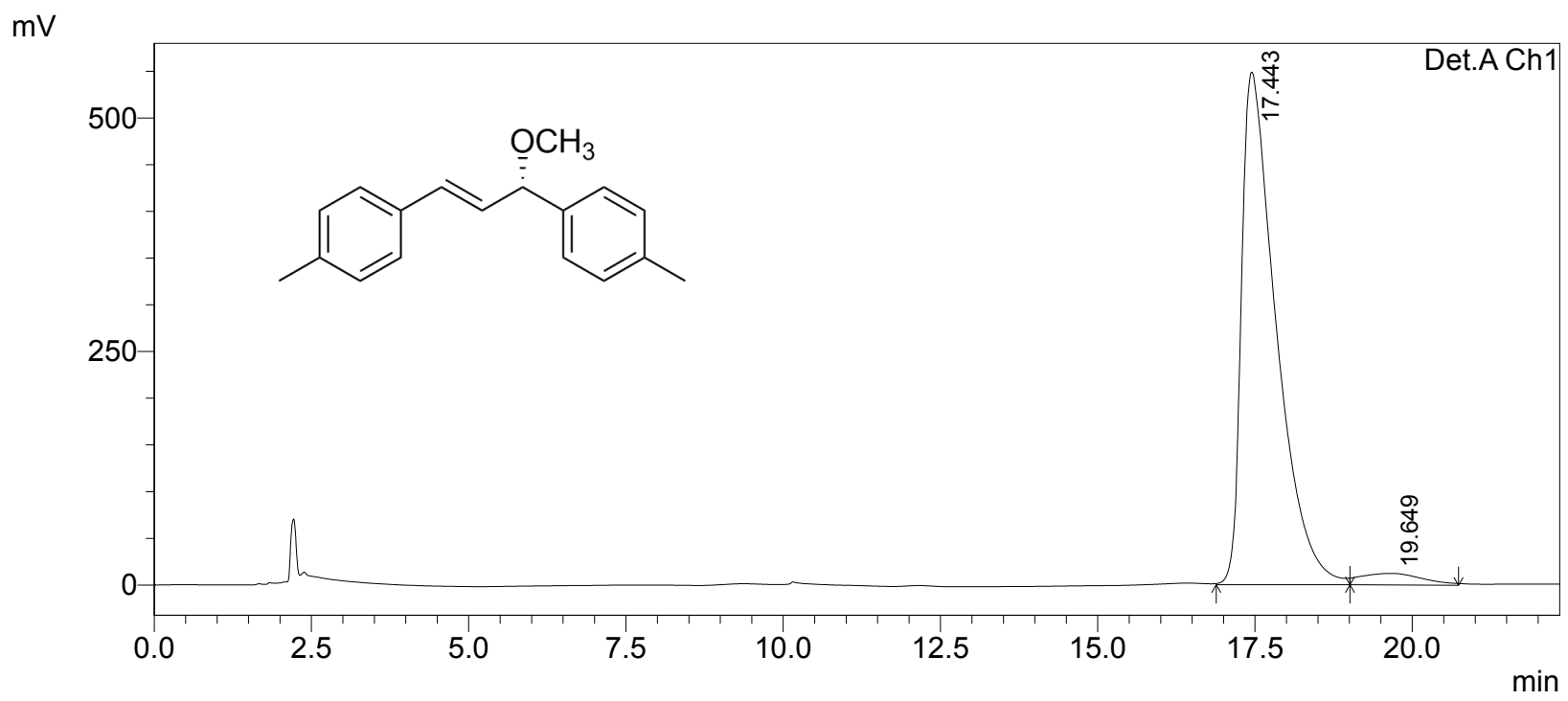

1 Det.A Ch1/254nm

PeakTable

Detector A Ch1 $254 \mathrm{~nm}$
\begin{tabular}{|r|r|r|r|r|r|}
\hline Peak\# & Ret. Time & \multicolumn{1}{c|}{ Area } & Height & Area $\%$ & \multicolumn{1}{c|}{ Height $\%$} \\
\hline 1 & 17.443 & 21048390 & 548698 & 96.157 & 97.801 \\
\hline 2 & 19.649 & 841303 & 12336 & 3.843 & 2.199 \\
\hline Total & & 21889694 & 561034 & 100.000 & 100.000 \\
\hline
\end{tabular}




\section{==== Analysis Report ====}

Acquired by

E:Idatal901ILSSILSS180727-3-XX(OJ 2\% 254 1)9.Icd : Admin

Sample Name

Sample ID

Vail \# : LSS180727-1(OJ 2\% 254 1)

: $1 \mathrm{uL}$

Injection Volume $\quad$ : LSS180727-3-XX(OJ 2\% 254 1)9.Icd

Data File Name : : 180327.Icm

Method File Name

Batch File Name

:

Default.Icr

: 2018/7/31 18:37:10

: 2018/7/31 19:06:17

Data Acquired

Data Processed

\section{$<$ Chromatogram $>$}

$\mathrm{mV}$

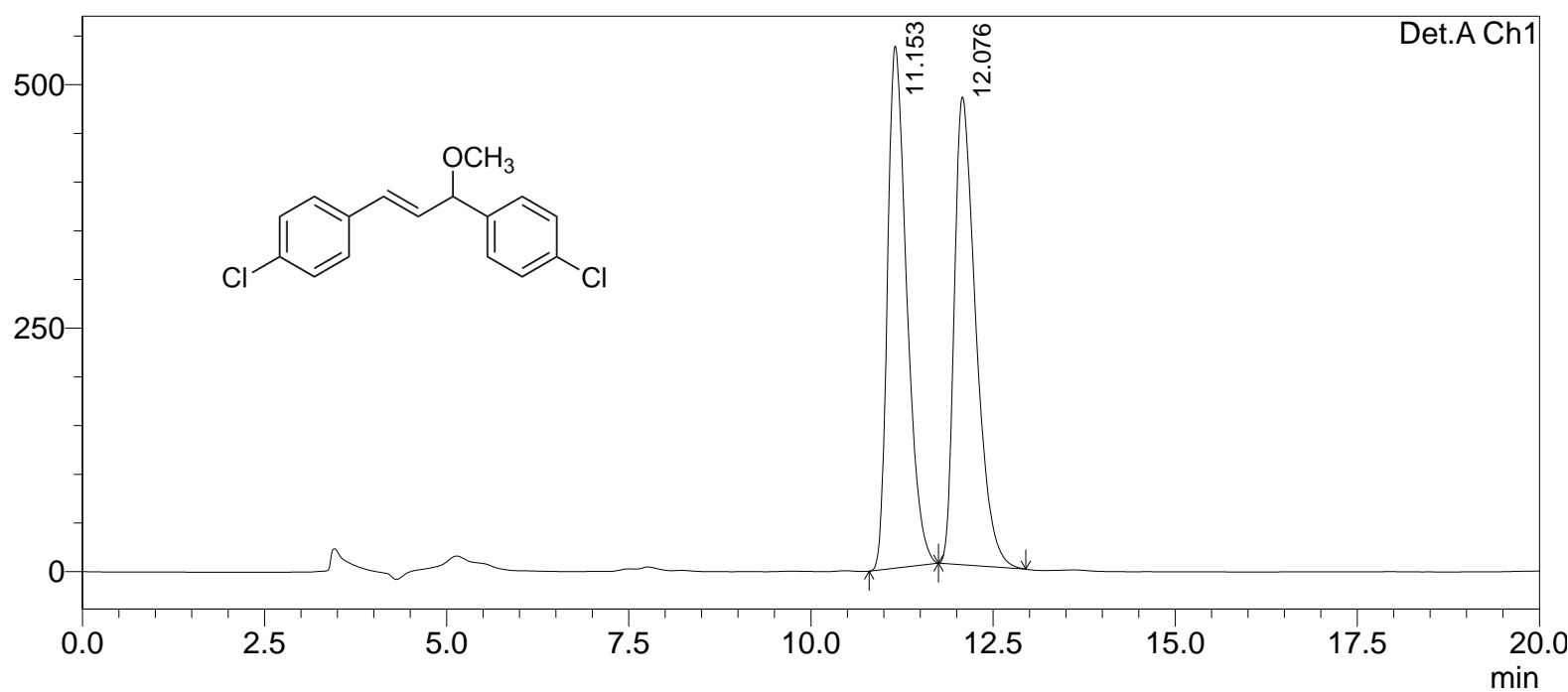

1 Det.A Ch1/260nm

PeakTable

Detector A Ch1 260nm

\begin{tabular}{|r|r|r|r|r|r|}
\hline \multicolumn{1}{|c|}{ Peak\# } & \multicolumn{1}{|c|}{ Ret. Time } & \multicolumn{1}{c|}{ Area } & \multicolumn{1}{c|}{ Height } & \multicolumn{1}{c|}{ Area \% } & \multicolumn{1}{c|}{ Height \% } \\
\hline 1 & 11.153 & 9861561 & 536195 & 49.970 & 52.756 \\
\hline 2 & 12.076 & 9873585 & 480168 & 50.030 & 47.244 \\
\hline Total & & 19735146 & 1016363 & 100.000 & 100.000 \\
\hline
\end{tabular}




\section{$== \pm=$ Analysis Report $====$}

Acquired by Sample Name

Sample ID

Vail \#

E:Idatal901ILSSILSS180727-1(OJ 2\% 254 1)10.Icd : Admin : LSS180727-1(OJ 2\% 254 1)

Injection Volume $\quad$ : LSS180727-1(OJ 2\% 254 1)10.Icd

Data File Name

Method File Name

Batch File Name

: $180327 . \mathrm{lcm}$

Report File Name

Data Acquired

: Default.lcr

: 2018/7/31 19:09:35

: 2018/7/31 19:29:36

Data Processed

\section{<Chromatogram>}

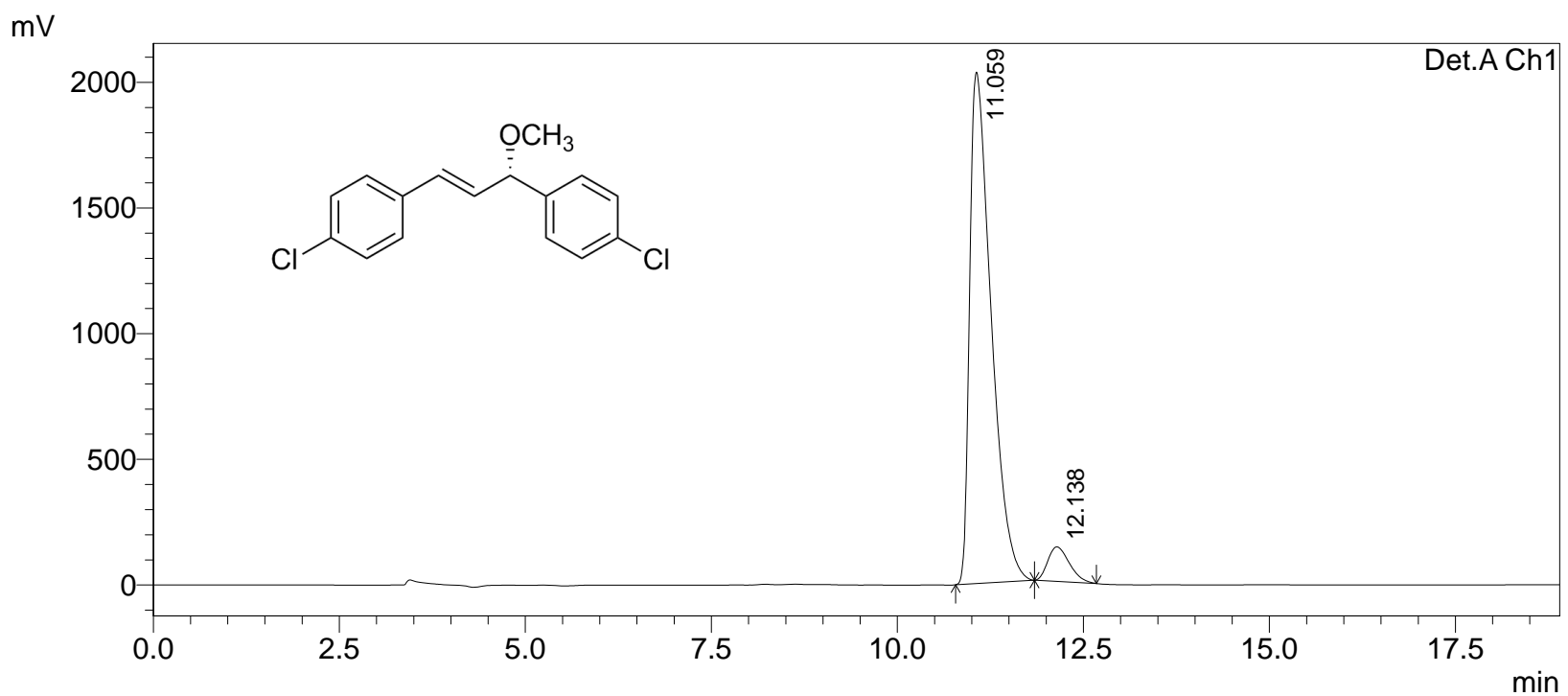

1 Det.A Ch1/260nm

PeakTable

Detector A Ch1 260nm
\begin{tabular}{|r|r|r|r|r|r|}
\hline Peak\# & Ret. Time & \multicolumn{1}{|c|}{ Area } & Height & Area $\%$ & Height $\%$ \\
\hline 1 & 11.059 & 40844824 & 2034846 & 93.636 & 93.667 \\
\hline 2 & 12.138 & 2775814 & 137569 & 6.364 & 6.333 \\
\hline Total & & 43620638 & 2172415 & 100.000 & 100.000 \\
\hline
\end{tabular}




\section{==== Analysis Report ====}

Acquired by

E:Idatal901ILSSILSS180730-3-XX(OD 2\% 254 1.0)1.Icd : Admin

Sample Name

Sample ID

Vail \# : LSS180730-3-XX(OD 2\% 254 1.0)

$: 1 \mathrm{uL}$

Injection Volume $\quad$ : LSS180730-3-XX(OD 2\% 254 1.0)1.Icd

Data File Name : : 180327.Icm

Method File Name

Batch File Name

Report File Name

Data Acquired :

- Default.Icr

: 2018/8/1 14:54:11

: 2018/8/1 15:18:43

Data Processed

\section{$<$ Chromatogram $>$}

$\mathrm{mV}$

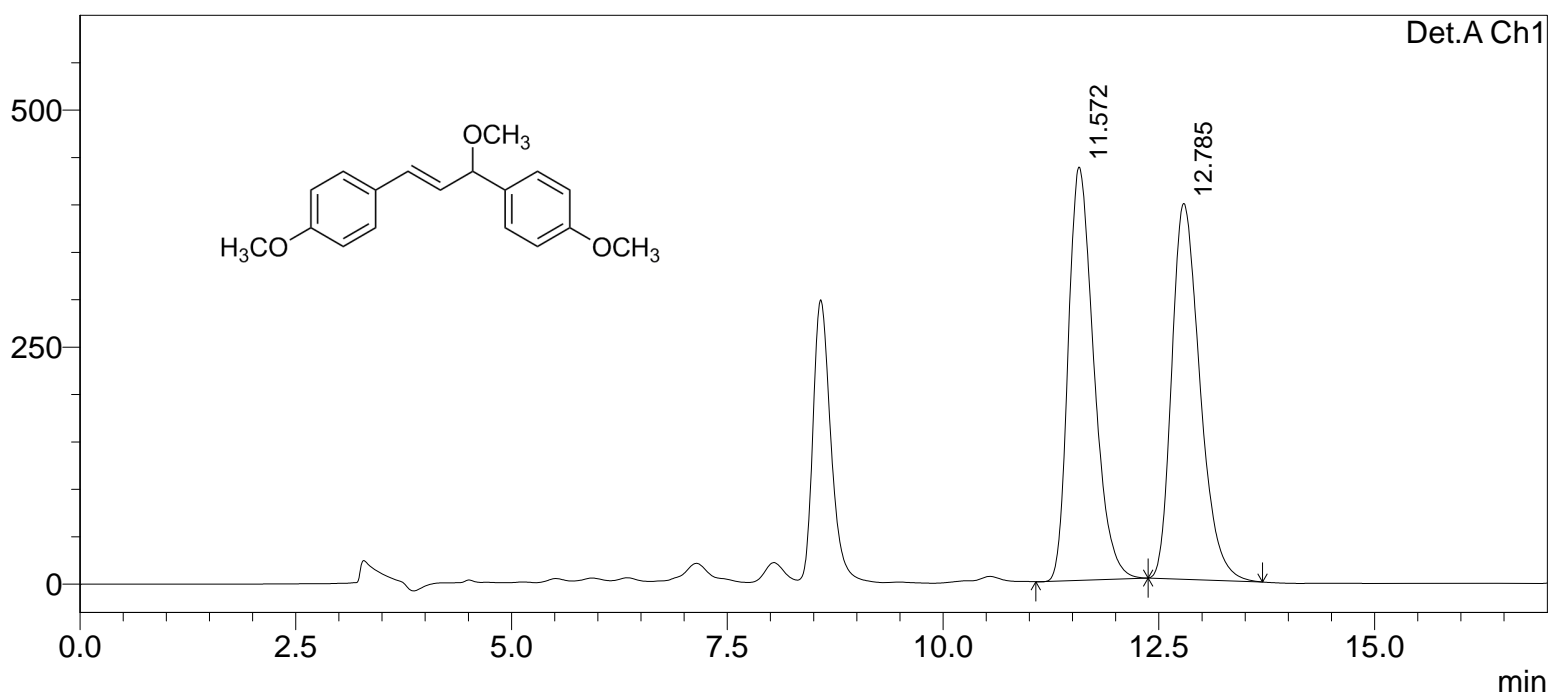

1 Det.A Ch1/254nm

PeakTable

Detector A Ch1 254nm

\begin{tabular}{|r|r|r|r|r|r|}
\hline Peak\# & Ret. Time & \multicolumn{1}{|c|}{ Area } & Height & \multicolumn{1}{|c|}{ Area \% } & \multicolumn{1}{|c|}{ Height \% } \\
\hline 1 & 11.572 & 8983678 & 436115 & 49.971 & 52.375 \\
\hline 2 & 12.785 & 8994153 & 396558 & 50.029 & 47.625 \\
\hline Total & & 17977831 & 832673 & 100.000 & 100.000 \\
\hline
\end{tabular}




\section{==== Analysis Report ====}

Acquired by Sample Name

Sample ID

Vail \#

E:Idatal901ILSSILSS180730-2(OD 2\% 254 1.0)2.Icd : Admin : LSS180730-2(OD 2\% 254 1.0)

Injection Volume $\quad$ : LSS180730-2(OD 2\% 254 1.0)2.Icd

Data File Name

Method File Name

Batch File Name

Report File Name

Data Acquired

: $180327 . \mathrm{Icm}$

Default.Icr

: 2018/8/1 15:25:44

: 2018/8/1 15:55:27

Data Processed

\section{$<$ Chromatogram>}

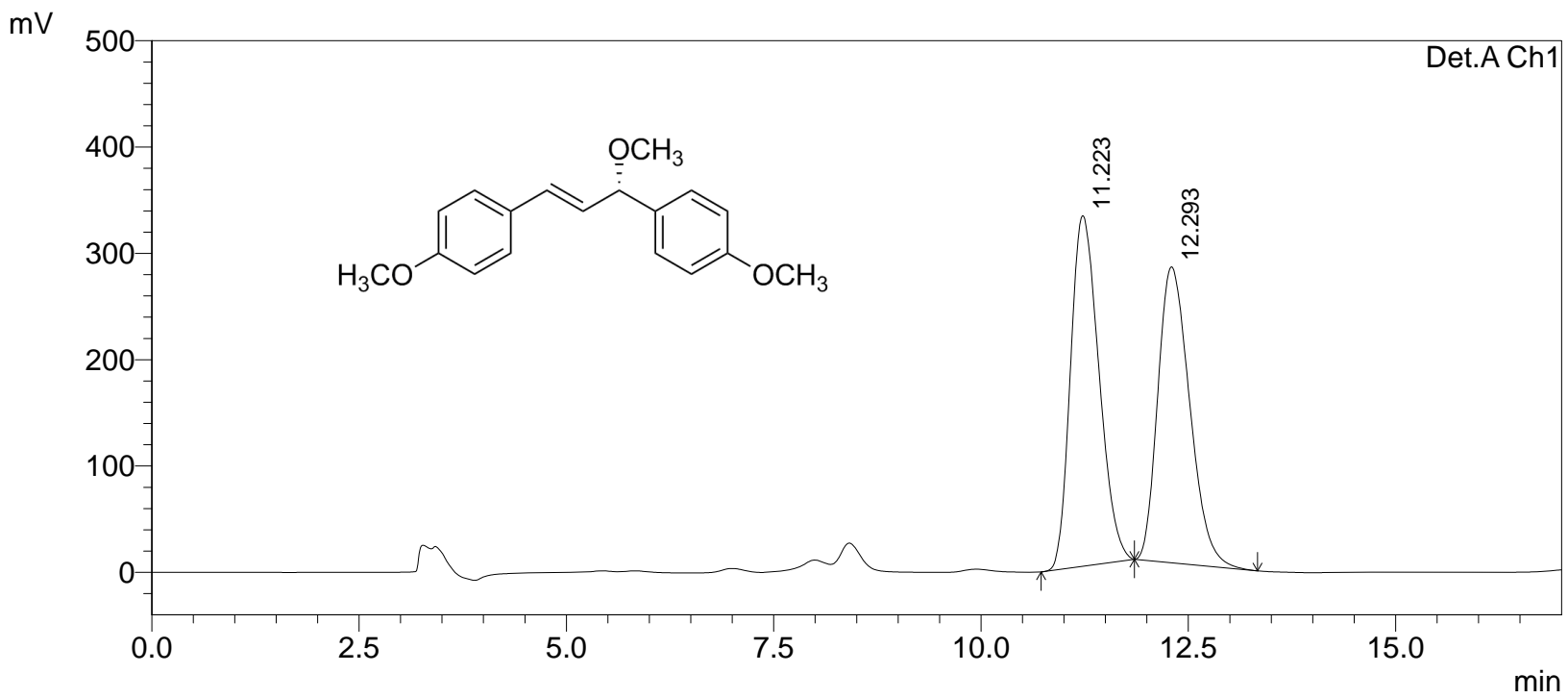

1 Det.A Ch1/254nm

Detector A Ch1 254nm

PeakTable

\begin{tabular}{|r|r|r|r|r|r|}
\hline Peak\# & Ret. Time & \multicolumn{1}{|c|}{ Area } & \multicolumn{1}{|c|}{ Height } & Area \% & \multicolumn{1}{c|}{ Height \% } \\
\hline 1 & 11.223 & 7866293 & 329802 & 51.405 & 54.231 \\
\hline 2 & 12.293 & 7436161 & 278345 & 48.595 & 45.769 \\
\hline Total & & 15302454 & 608146 & 100.000 & 100.000 \\
\hline
\end{tabular}




\section{$===$ Analysis Report $====$}

Acquired by

Sample Name

Sample ID

Vail \#

Injection Volume

Data File Name

Method File Name

Batch File Name

Report File Name

Data Acquired

Data Processed
E:Idatal901ILSSILSS180917-1-XX(AD 5\% 1254 )

: LSS180917-1-XX(AD 5\% 1 254)

: $1 \mathrm{uL}$

: LSS180917-1-XX(AD 5\% 1 254)

: 180327.Icm

: Default.Icr

: 2018/9/18 15:44:10

: 2018/9/18 16:01:09

\section{$<$ Chromatogram >}

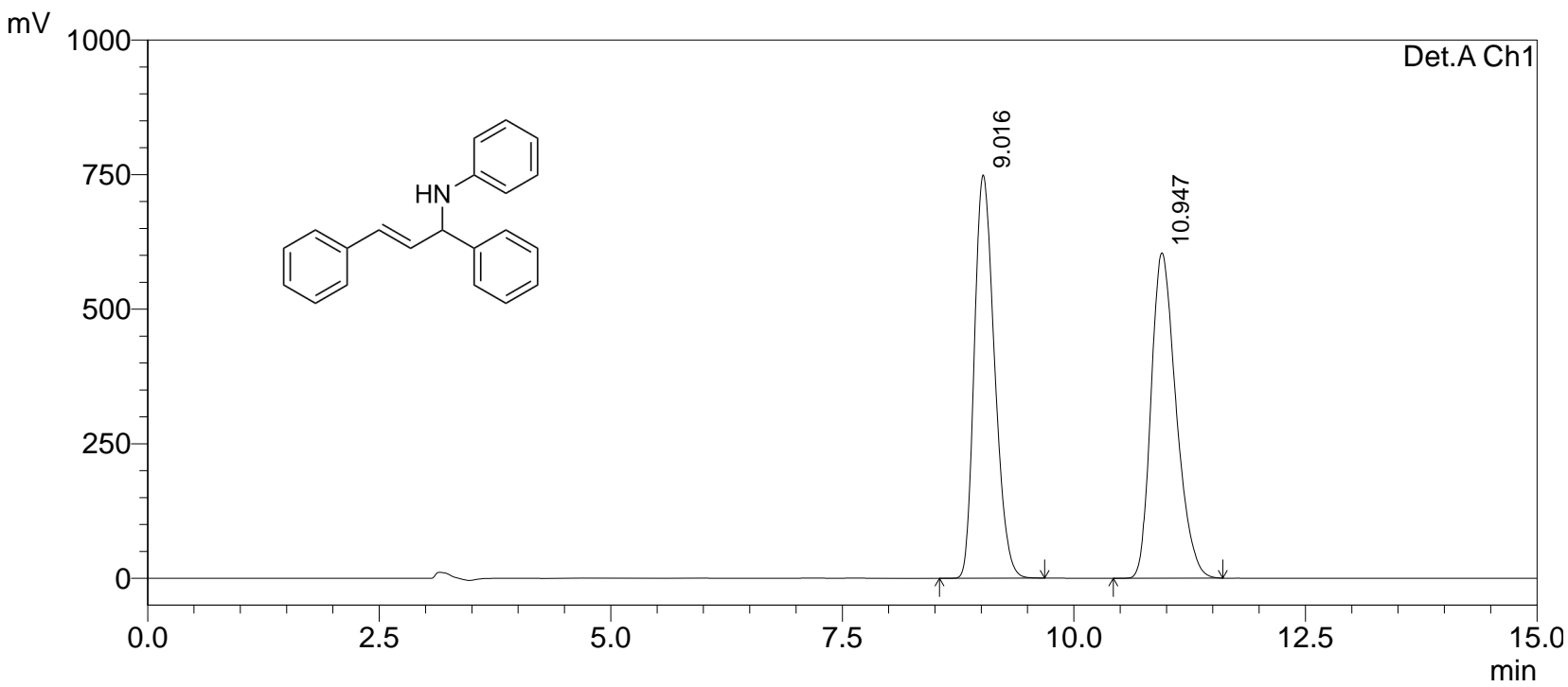

1 Det.A Ch1/254nm

Detector A Ch1 254nm

PeakTable

\begin{tabular}{|r|r|r|r|r|r|}
\hline \multicolumn{1}{|c|}{ Peak\# } & Ret. Time & \multicolumn{1}{|c|}{ Area } & \multicolumn{1}{c|}{ Height } & \multicolumn{1}{c|}{ Area \% } & \multicolumn{1}{c|}{ Height \% } \\
\hline 1 & 9.016 & 11350977 & 749401 & 49.990 & 55.345 \\
\hline 2 & 10.947 & 11355563 & 604643 & 50.010 & 44.655 \\
\hline Total & & 22706539 & 1354044 & 100.000 & 100.000 \\
\hline
\end{tabular}




\section{=ニニ= Analysis Report $====$}

Acquired by Sample Name

Sample ID

Vail \#

E:Idatal901ILSSILSS180917-1(AD 5\% 1 254): Admin : LSS180917-1(AD 5\% 1 254)

$: 1 \mathrm{uL}$

Injection Volume $\quad$ : LSS180917-1(AD 5\% 1254$)$

Data File Name : : 180327.Icm

Method File Name

Batch File Name

Report File Name

Data Acquired :

: Default.Icr

: 2018/9/19 8:52:59

: 2018/9/19 9:11:07

Data Processed

\section{$<$ Chromatogram >}

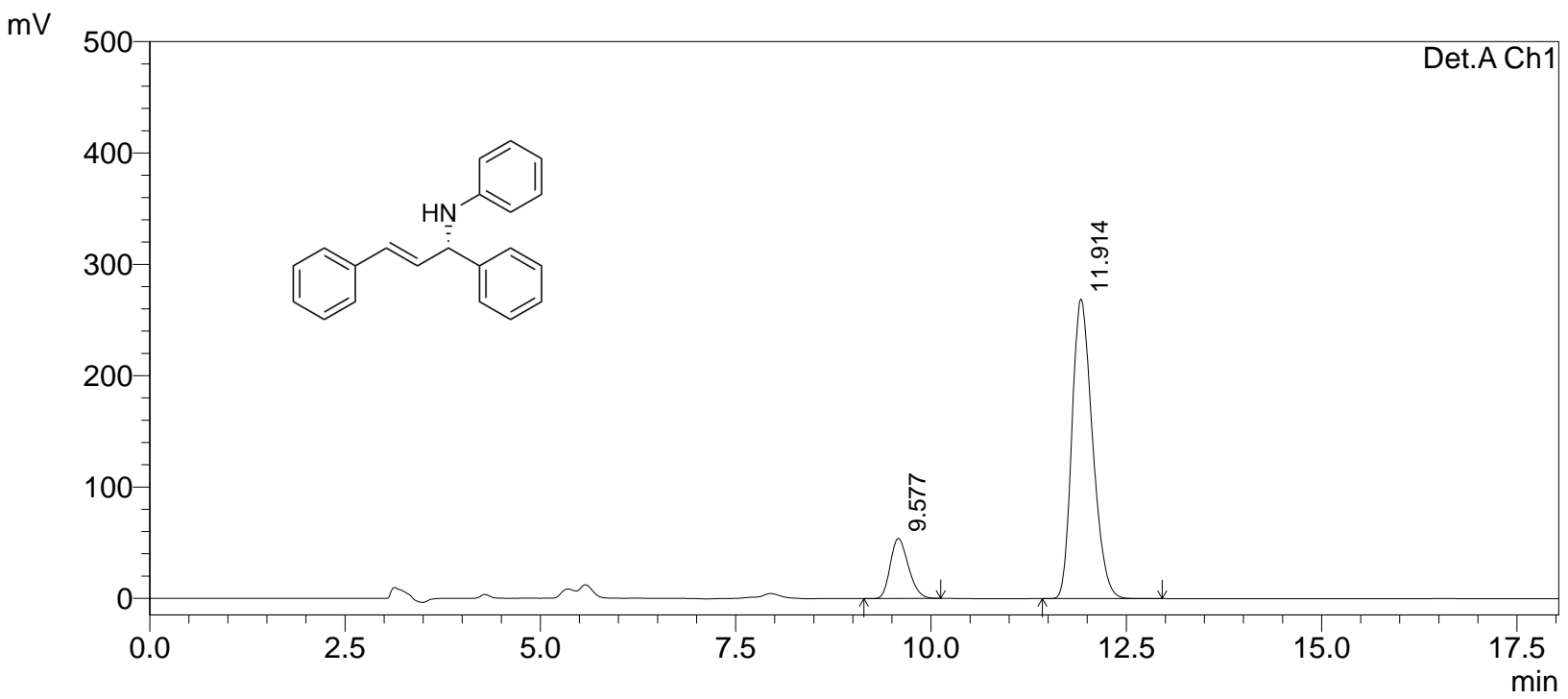

1 Det.A Ch1/254nm

PeakTable

Detector A Ch1 254nm

\begin{tabular}{|r|r|r|r|r|r|}
\hline Peak\# & Ret. Time & \multicolumn{1}{|c|}{ Area } & Height & \multicolumn{1}{|c|}{ Area \% } & Height \% \\
\hline 1 & 9.577 & 868964 & 53983 & 14.989 & 16.706 \\
\hline 2 & 11.914 & 4928405 & 269150 & 85.011 & 83.294 \\
\hline Total & & 5797368 & 323133 & 100.000 & 100.000 \\
\hline
\end{tabular}




\section{==== Analysis Report ====}

Acquired by Sample Name

Sample ID

Vail \#

E:Idatal901ILSSILSS180912-5-XX(OJ 30\% 1 254) : Admin

: LSS180912-5-XX(OJ 30\% 1254$)$

$: 1 \mathrm{uL}$

Injection Volume $\quad$ : LSS180912-5-XX(OJ 30\% 1254 )

Data File Name

Method File Name

Batch File Name

Report File Name

Data Acquired

: 180327.Icm

: Default.Icr

: 2018/9/14 8:55:08

: 2018/9/14 9:30:52

Data Processed

\section{$<$ Chromatogram >}

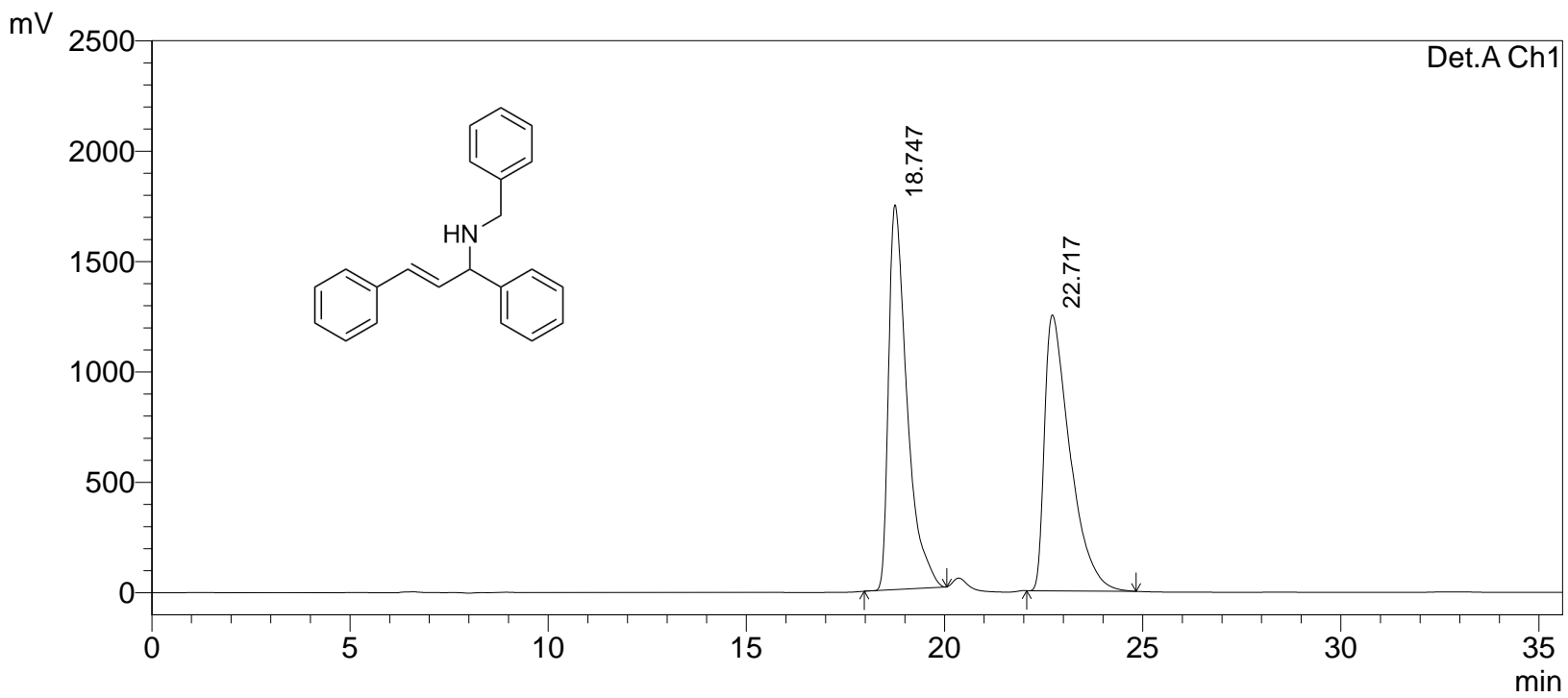

1 Det.A Ch1/254nm

Detector A Ch1 254nm
\begin{tabular}{|r|r|r|r|r|r|}
\hline \multicolumn{1}{|c|}{ Peak\# } & Ret. Time & \multicolumn{1}{|c|}{ Area } & Height & Area $\%$ & \multicolumn{1}{c|}{ Height $\%$} \\
\hline 1 & 18.747 & 56273068 & 1742952 & 50.220 & 58.245 \\
\hline 2 & 22.717 & 55780739 & 1249478 & 49.780 & 41.755 \\
\hline Total & & 112053807 & 2992430 & 100.000 & 100.000 \\
\hline
\end{tabular}




\section{$====$ Analysis Report $====$}

Acquired by Sample Name Sample ID

Vail \#

E:Idatal901ILSSILSS180907-(5)(OJ 13\% 0.5 254) : Admin : LSS180907-(5)(OJ 13\% 0.5 254)

$: 1 \mathrm{uL}$

Injection Volume $\quad:$ LSS180907-(5)(OJ 13\% 0.5 254)

Data File Name : : 180327.Icm

Method File Name

Batch File Name

Report File Name

Data Acquired :

: Default.Icr

: 2018/9/15 15:56:09

: 2018/9/15 16:27:23

Data Processed

\section{$<$ Chromatogram $>$}

$\mathrm{mV}$

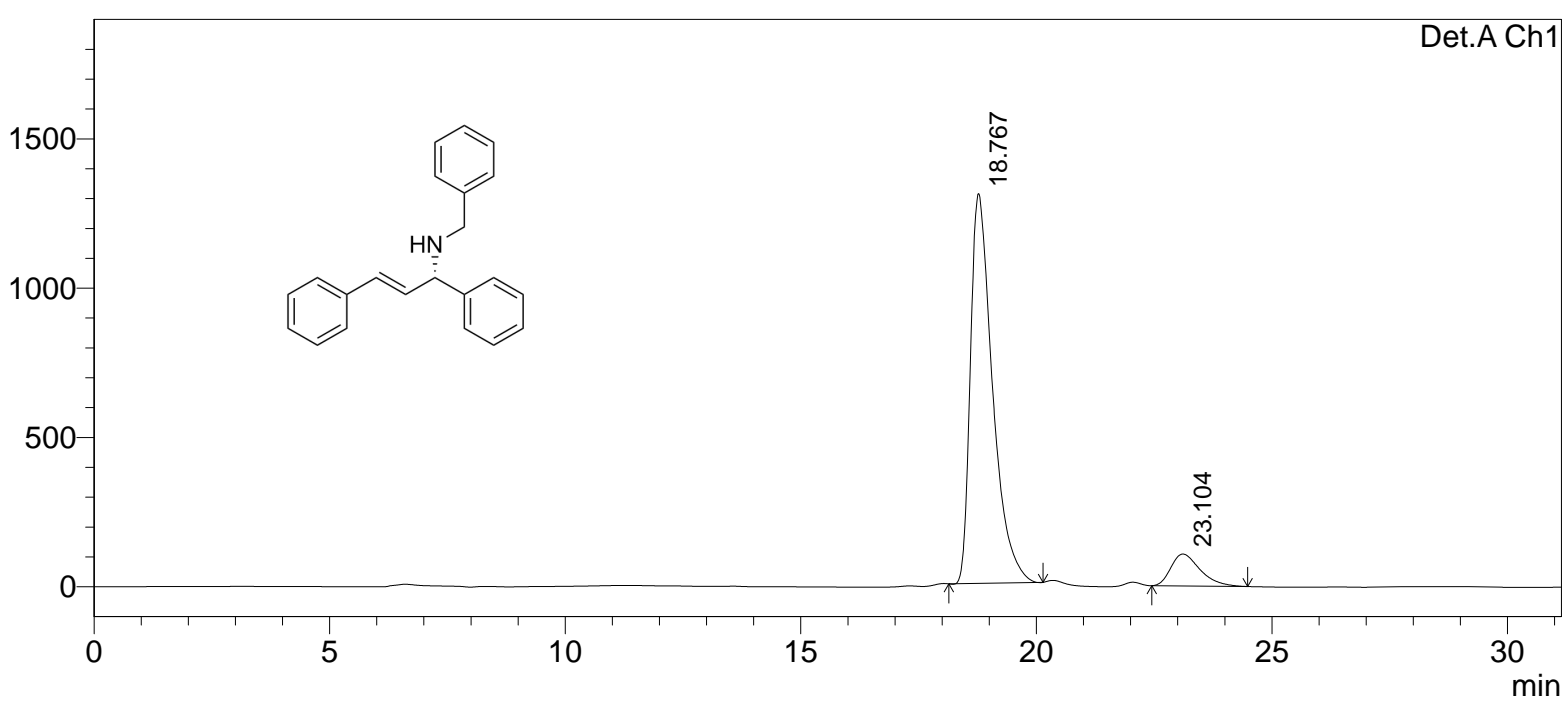

1 Det.A Ch1/254nm

PeakTable

Detector A Ch1 254nm

\begin{tabular}{|r|r|r|r|r|r|}
\hline \multicolumn{1}{|c|}{ Peak\# } & Ret. Time & \multicolumn{1}{|c|}{ Area } & \multicolumn{1}{|c|}{ Height } & Area \% & \multicolumn{1}{c|}{ Height \% } \\
\hline 1 & 18.767 & 42541803 & 1305426 & 90.193 & 92.424 \\
\hline 2 & 23.104 & 4625954 & 107004 & 9.807 & 7.576 \\
\hline Total & & 47167757 & 1412430 & 100.000 & 100.000 \\
\hline
\end{tabular}




\section{==== Analysis Report ====}

Acquired by Sample Name

E:Idatal901ILSSILTY180930-2-XX(OD 2\% 1 254)

: Admin

: LTY180930-2-XX(OD 2\% 1254$)$

Sample ID

Vail \#

Injection Volume

Data File Name

Method File Name

Batch File Name

Report File Name

Data Acquired

$: 1 \mathrm{uL}$

: LTY180930-2-XX(OD 2\% 1 254) : 180327.Icm

: Default.lcr

: 2018/10/1 14:08:04

: 2018/10/1 15:58:18

\section{$<$ Chromatogram >}

$\mathrm{mV}$

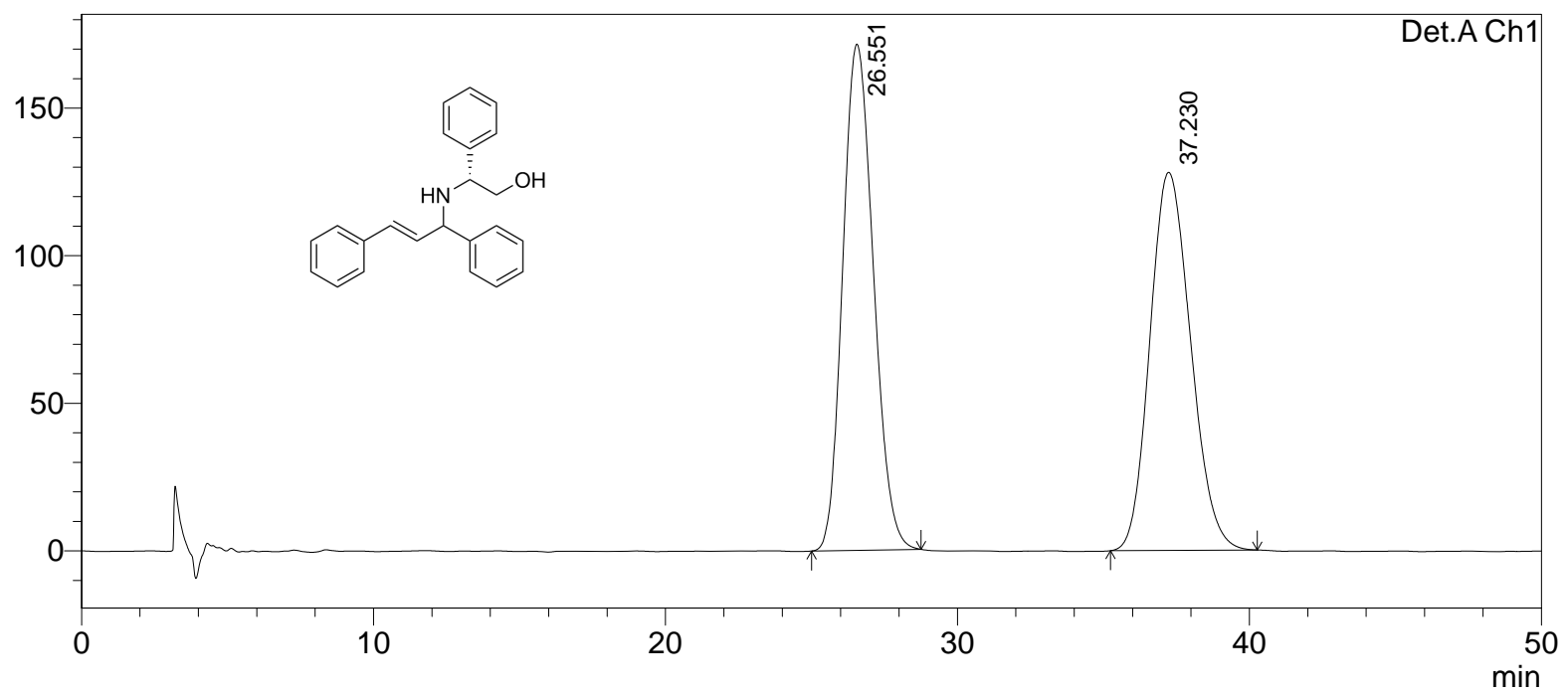

1 Det.A Ch1/260nm

PeakTable

Detector A Ch1 260nm

\begin{tabular}{|r|r|r|r|r|r|}
\hline Peak\# & Ret. Time & Area & Height & \multicolumn{1}{|c|}{ Area \% } & \multicolumn{1}{c|}{ Height \% } \\
\hline 1 & 26.551 & 12527596 & 171500 & 50.203 & 57.244 \\
\hline 2 & 37.230 & 12426307 & 128095 & 49.797 & 42.756 \\
\hline Total & & 24953903 & 299595 & 100.000 & 100.000 \\
\hline
\end{tabular}




\section{$====$ Analysis Report $====$}

Acquired by Sample Name Sample ID

Vail \#

Injection Volume

Data File Name

Method File Name

Batch File Name

Report File Name

Data Acquired

Data Processed
E:Idatal901LSSILSS180930-2(OD 2\% 1 254)

: LSS180930-2(OD 2\% 1254 )

: $1 \mathrm{uL}$

: LSS180930-2(OD 2\% 1 254)

: $180327 . \mathrm{Icm}$

: Default.lcr

2018/10/2 15:58:13

: 2018/10/2 16:50:18

\section{$<$ Chromatogram >}

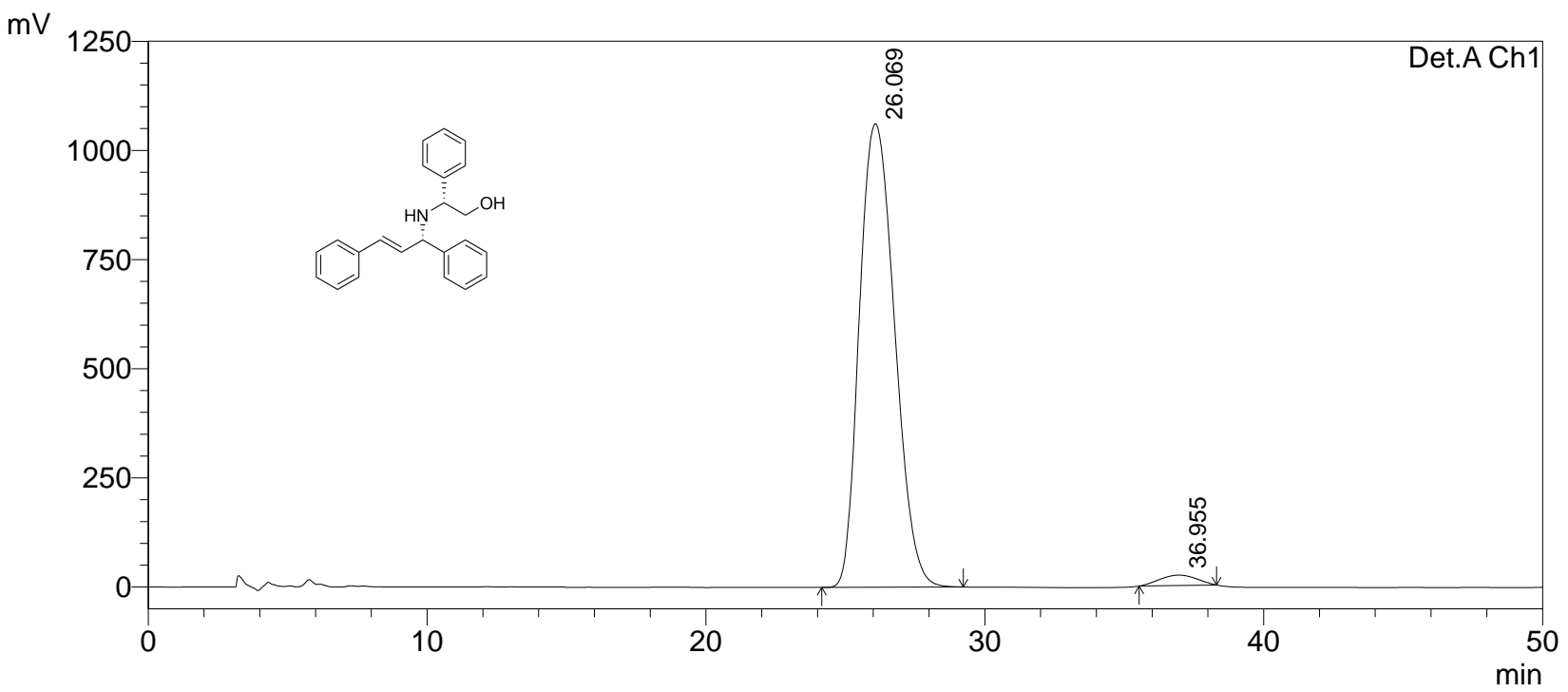

1 Det.A Ch1/260nm

PeakTable

Detector A Ch1 260nm

\begin{tabular}{|r|r|r|r|r|r|}
\hline \multicolumn{1}{|c|}{ Peak\# } & \multicolumn{1}{|c|}{ Ret. Time } & \multicolumn{1}{c|}{ Area } & \multicolumn{1}{c|}{ Height } & Area \% & \multicolumn{1}{c|}{ Height \% } \\
\hline 1 & 26.069 & 93265169 & 1061750 & 97.706 & 97.818 \\
\hline 2 & 36.955 & 2189996 & 23689 & 2.294 & 2.182 \\
\hline Total & & 95455165 & 1085438 & 100.000 & 100.000 \\
\hline
\end{tabular}




\section{=ニニ= Analysis Report $====$}

Acquired by Sample Name

Sample ID

Vail \#

E:Idatal901LSSILTY181013-L-XX(OD 2\% 1 254)2.Icd : Admin : LTY181013-L-XX(OD 2\% 1254$)$

: $1 \mathrm{uL}$

Injection Volume $\quad$ : LTY181013-L-XX(OD 2\% 1 254)2.Icd

Data File Name

Method File Name

Batch File Name

Report File Name

Data Acquired : 180327.Icm

: Default.Icr

: 2018/10/16 20:55:51

: 2018/10/16 21:42:04

Data Processed

\section{$<$ Chromatogram>}

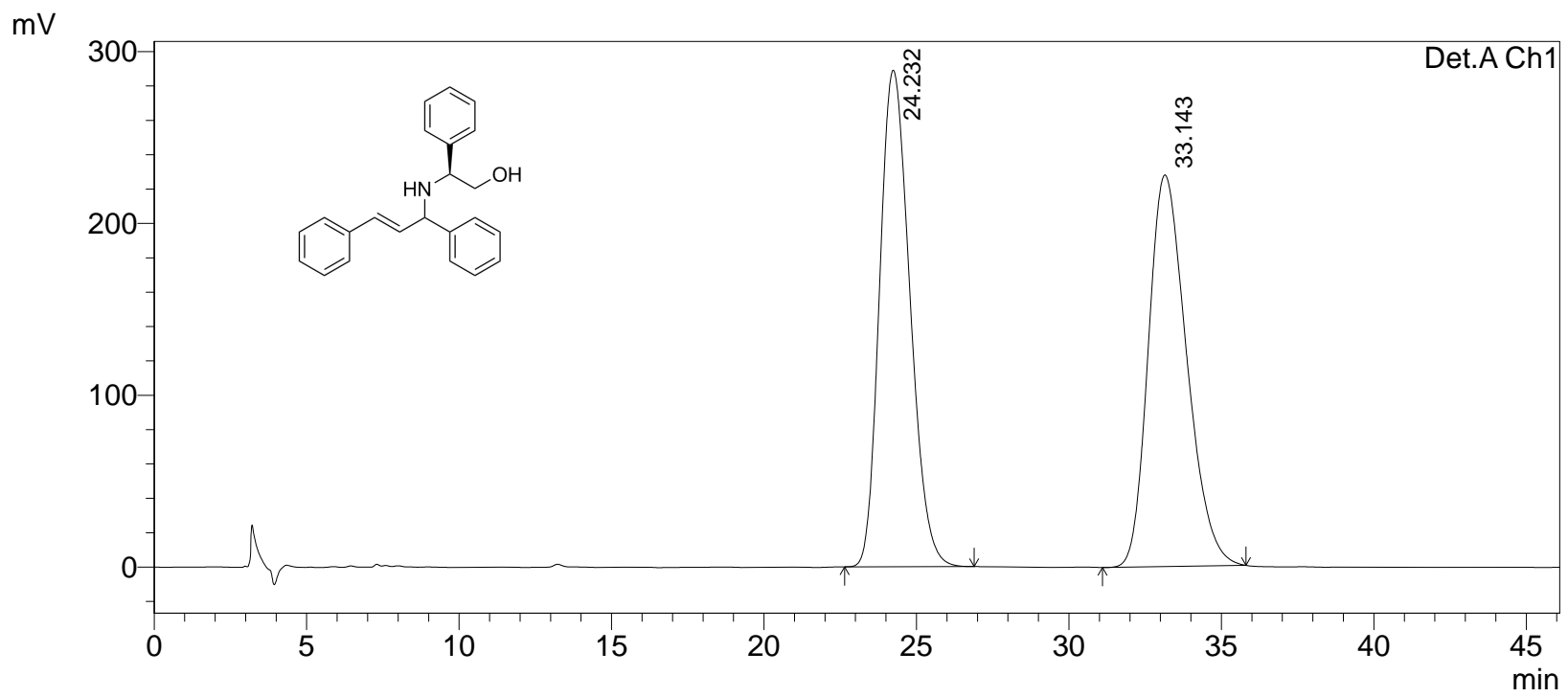

1 Det.A Ch1/254nm

PeakTable

Detector A Ch1 254nm

\begin{tabular}{|r|r|r|r|r|r|}
\hline \multicolumn{1}{|c|}{ Peak\# } & Ret. Time & \multicolumn{1}{|c|}{ Area } & Height & Area \% & \multicolumn{1}{c|}{ Height \% } \\
\hline 1 & 24.232 & 19967190 & 289053 & 50.072 & 55.906 \\
\hline 2 & 33.143 & 19909798 & 227982 & 49.928 & 44.094 \\
\hline Total & & 39876988 & 517035 & 100.000 & 100.000 \\
\hline
\end{tabular}




\section{==== Analysis Report ====}

Acquired by Sample Name

Sample ID

Vail \#

E:Idatal901ILSSILSS181009-L(OD 2\% 1.0 254)1.Icd : Admin : LSS181009-L(OD 2\% 1.0 254)

Injection Volume $\quad$ : LSS181009-L(OD 2\% 1.0 254)1.Icd

Data File Name

Method File Name

Batch File Name

Report File Name

Data Acquired

: $180327 . \mathrm{Icm}$

Default.Icr

: 2018/10/12 9:28:30

: 2018/10/12 10:20:15

Data Processed

\section{$<$ Chromatogram $>$}

$\mathrm{mV}$

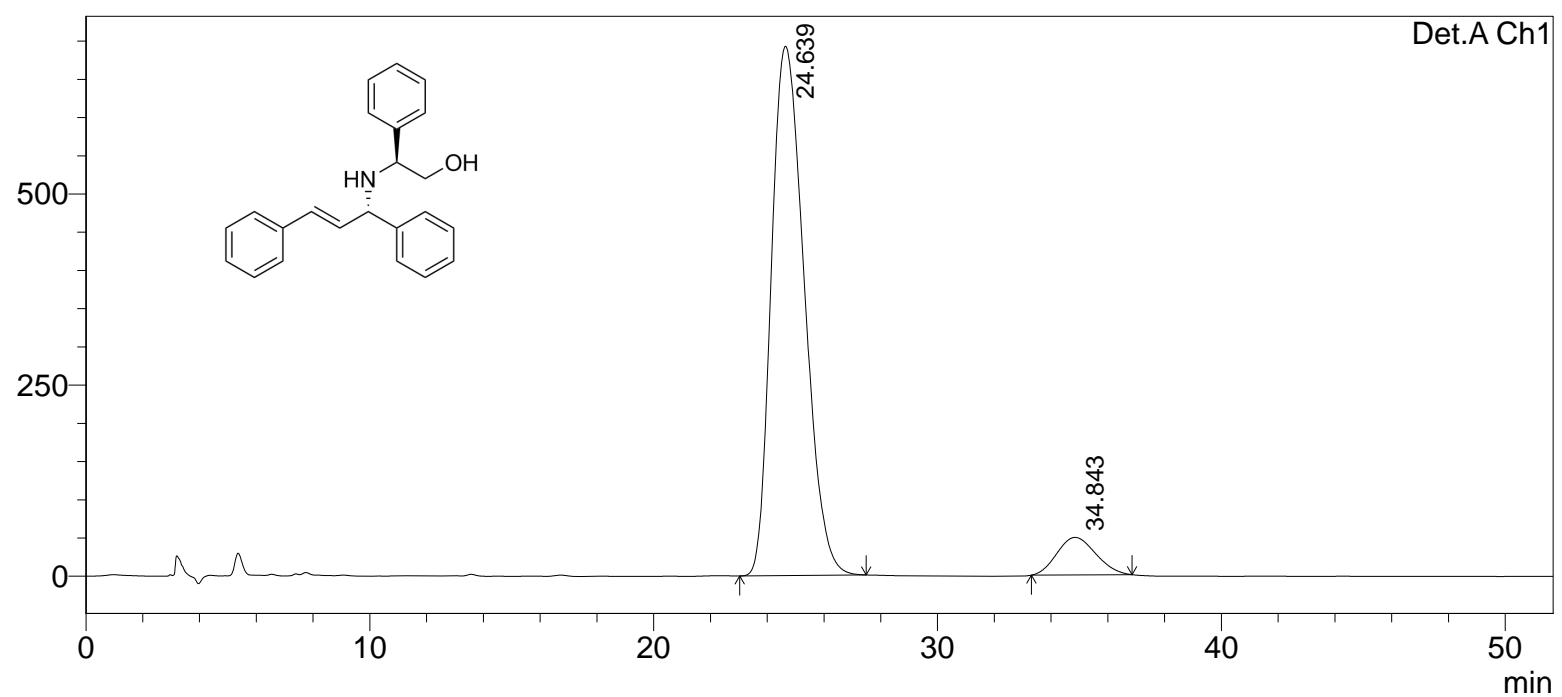

1 Det.A Ch1/254nm

PeakTable

Detector A Ch1 254nm

\begin{tabular}{|r|r|r|r|r|r|}
\hline \multicolumn{1}{|c|}{ Peak\# } & \multicolumn{1}{|c|}{ Ret. Time } & \multicolumn{1}{c|}{ Area } & \multicolumn{1}{c|}{ Height } & Area \% & \multicolumn{1}{c|}{ Height \% } \\
\hline 1 & 24.639 & 55830780 & 692414 & 92.269 & 93.374 \\
\hline 2 & 34.843 & 4678212 & 49132 & 7.731 & 6.626 \\
\hline Total & & 60508991 & 741545 & 100.000 & 100.000 \\
\hline
\end{tabular}

